\title{
UICN
}

Les Zones clés pour la biodiversité d'eau douce dans le hotspot du bassin méditerranéen

Un éclairage pour la conservation des espèces et la planification du développement dans les écosystèmes d'eau douce

Darwall W., Carrizo S., Numa C., Barrios V., Freyhof J. et Smith K. 
La terminologie géographique employée dans cet ouvrage, de même que sa présentation, ne sont en aucune manière l'expression d'une opinion quelconque de la part de I'UICN ou des autres organisations concernées sur le statut juridique ou l'autorité de quelque pays, territoire ou région que ce soit, ou sur la délimitation de ses frontières.

Les opinions exprimées dans cette publication ne reflètent pas nécessairement celles de l'UICN ou des autres organisations concernées.

L'UICN et les autres organisations concernées rejettent toute responsabilité en cas d'erreurs ou d'omissions intervenues lors de la traduction en français de ce document dont la version originale est en anglais.

Publié par: $\quad$ UICN, Gland, Suisse, Cambridge, Royaume-Uni et Malaga, Espagne

Droits d'auteur : (c) 2015 Union internationale pour la conservation de la nature et de ses ressources

La reproduction de cette publication à des fins commerciales, notamment en vue de la vente, est interdite sans autorisation écrite préalable du détenteur des droits d'auteur à condition que la source soit dûment citée.

Citation : Darwall W., Carrizo S., Numa C., Barrios V., Freyhof J. et Smith K. (2015). Les Zones clés pour la biodiversité d'eau douce dans le hotspot du bassin méditerranéen. Un éclairage pour la conservation des espèces et la planification du développement dans les écosystèmes d'eau douce. Cambridge, Royaume-Uni et Malaga, Espagne : UICN. $\mathrm{x}+86 \mathrm{pp}$.

ISBN : 978-2-8317-1708-1

DOI : 10.2305/IUCN.CH.2015.SSC-OP.52.fr

Photo couverture : Affluent du lac Skadar (Albanie et Monténégro). Ce vaste lac méditerranéen, ainsi que son bassin versant associé, est une Zone clé pour la biodiversité (ZCB) d'eau douce, abritant au moins 24 espèces parmi les espèces d'eau douce menacées ou ayant une aire de répartition restreinte. @ C Geert De Knijf.

Toutes les photographies utilisées dans cette publication restent la propriété du détenteur des droits d'auteur d'origine (voir légendes individuelles pour plus de précisions). Les photographies ne doivent pas être reproduites ou utilisées dans d'autres contextes sans l'autorisation écrite du détenteur des droits d'auteur.

Mise en page : NatureBureau

Traduction : Alexa Dubreuil-Storer

Imprimé par : Solprint, Mijas (Malaga), Spain

Disponible auprès de :

Centre de Coopération pour la Méditerranée de l'UICN

$\mathrm{C} /$ Marie Curie 22

29590 Campanillas

Malaga, Espagne

Tel: +34 952028430

Fax: +34 952028145

www.iucn.org/mediterranean

www.iucn.org/publications

Le présent ouvrage a pu être publié grâce à un soutien financier du Fonds de Partenariat pour les Écosystèmes Critiques (CEPF) et de la Fondation MAVA.

Le Fonds de Partenariat pour les Écosystèmes Critiques (CEPF) est une initiative conjointe de l'Agence Française de Développement (AFD), de la Banque mondiale, de Conservation International, de la Fondation MacArthur, du Fonds pour l'environnement mondial (FEM), du Gouvernement Japonais et de l'Union européenne. L'un des objectifs fondamentaux est de garantir la participation de la société civile dans la conservation de la biodiversité.

Si vous avez des questions concernant les données et les résultats présentés dans ce rapport, veuillez contacter l'Unité de biodiversité d'eau douce de l'UICN (Freshwater.Biodiversity@iucn.org). 


\section{Les Zones clés pour la biodiversité d'eau douce dans le hotspot du bassin méditerranéen}

\section{Un éclairage pour la conservation des espèces et la planification du développement dans les écosystèmes d'eau douce}

Darwall W., Carrizo S., Numa C., Barrios V., Freyhof J. et Smith K. 



\section{Table des matières}

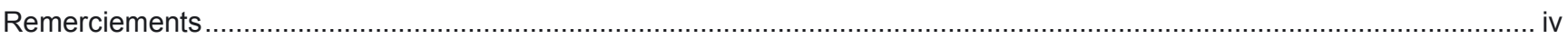

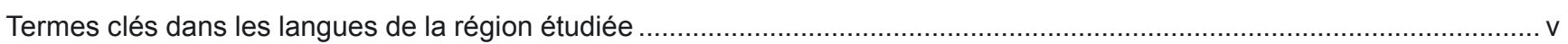

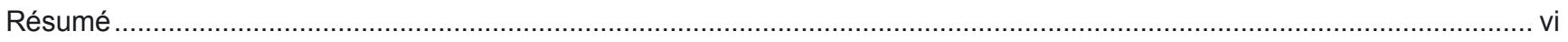

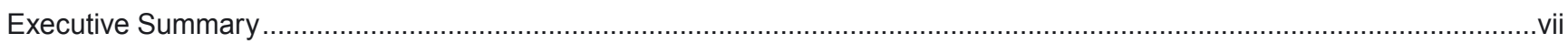

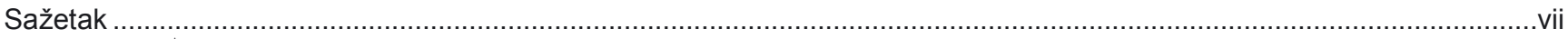

ملخص تنفيذي

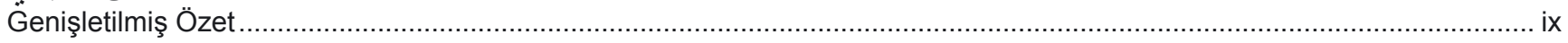

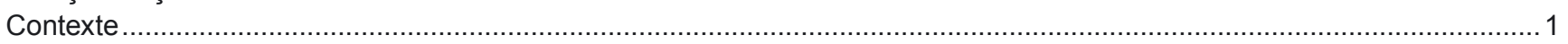

Le statut de la biodiversité d'eau douce dans le bassin méditerranéen ............................................................... 1

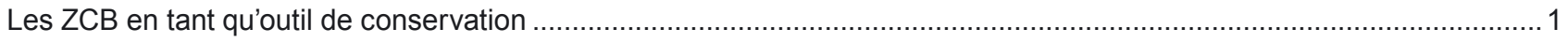

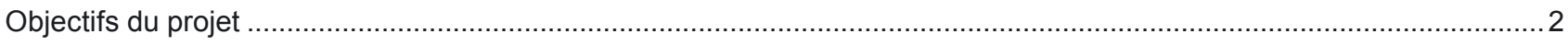

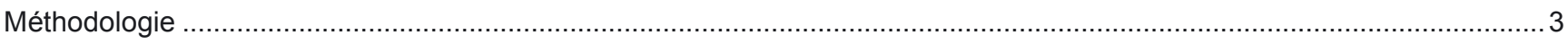

Critères d'identification des Zones clés pour la biodiversité (ZCB) d'eau douce ................................................... 3

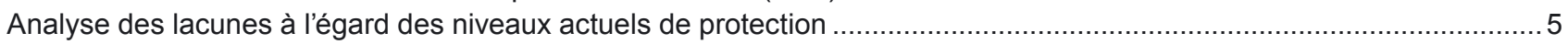

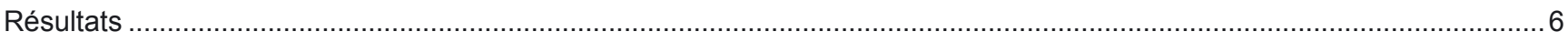

Identification des sous-bassins versants fluviaux/lacustres répondant aux critères d'admission en tant que

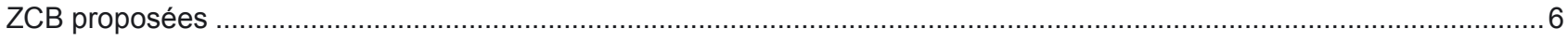

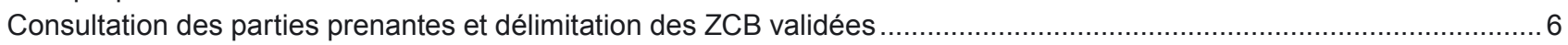

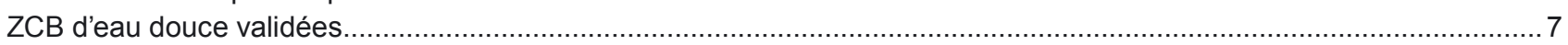

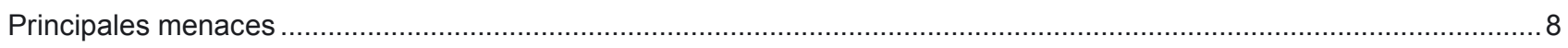

Niveaux actuels de protection des ZCB d'eau douce au sein du hotspot méditerranéen ........................................... 8

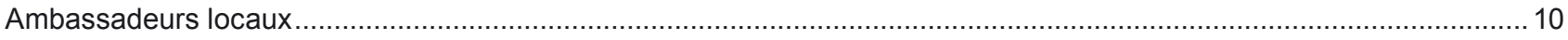

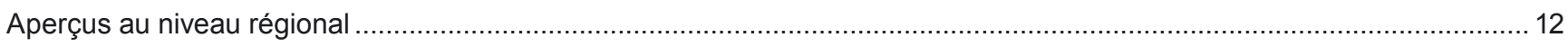

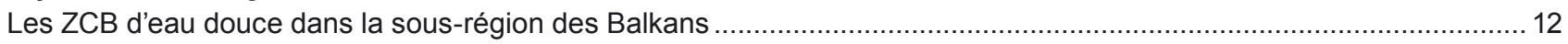

Les ZCB d'eau douce dans la sous-région de la Turquie et du Levant ................................................................... 21

Les ZCB d'eau douce dans la sous-région de l'Afrique du Nord ....................................................................... 30

Les ZCB d'eau douce dans la sous-région du nord-ouest de la Méditerranée........................................................38

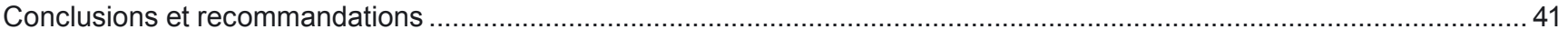

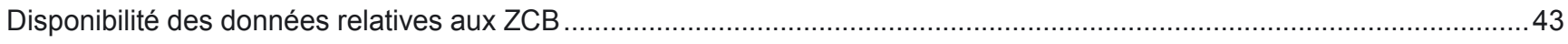

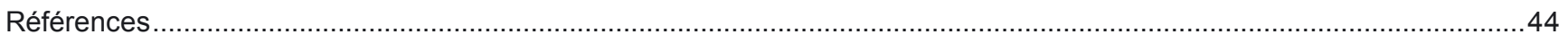

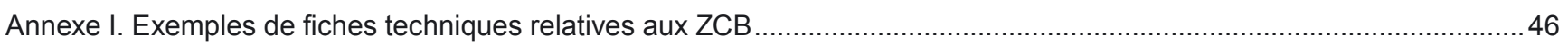

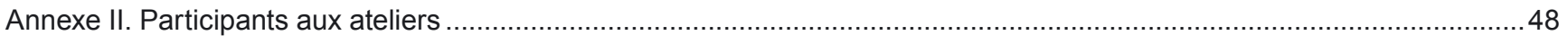

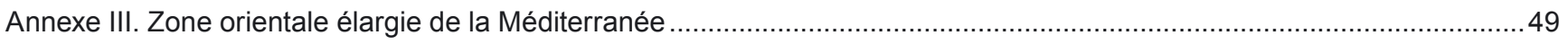

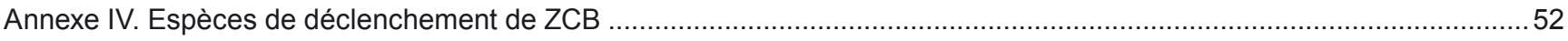

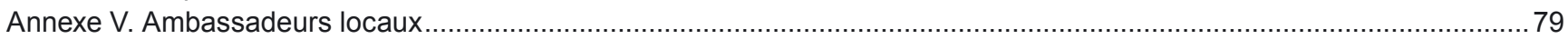

Annexe VI. ZCB proposées en raison de plantes et d'odonates de la sous-région de la Turquie et du Levant ......................86 


\section{Remerciements}

L'identification des Zones clés pour la biodiversité dans la région méditerranéenne repose sur la volonté d'experts dédiés et désireux d'apporter leur contribution et de mettre en commun leurs connaissances. Sans cet engagement et cet enthousiasme en faveur de la conservation de la biodiversité, ce travail ne serait pas possible. Ainsi, nous souhaitons remercier tous les experts méditerranéens qui ont participé aux ateliers organisés en Bosnie-Herzégovine, au Maroc et en Jordanie, comme Aljoša Duplić, Dejan Kulijer, Drazen Kotrosan, Geert De Knijf, loannis Bazos, Jörg Freyhof, Kanella Radea, Krešimir Žganec, Marko Ćaleta, Panagiota Maragou, Stamatis Zogaris, Ulrich Eichelman, Zoran Marčić, Zoran Mateljak, Güler Ekmekci, Nashat Hamidan, Ümit Kebapçi, Manuel Lopes Lima, Dirk Van Damme, Zuhair Amr, Süreyya İsfendiyaroğlu, Haifaa Abdulhalim, Cagri Gocek, Laith El Moghrabi, Imtinen Ben Hadj Jilani, Miguel Clavero, Mohamed Dakki, Mohamed Ghamizi, Mohammed Melhaoui, Mohamed Noaman, Laila Rhazi, Boudjéma Samraoui et Jamel Tahri.
Nous remercions tout particulièrement Arantxa Cendoya pour son soutien dans le cadre de l'organisation logistique des ateliers et de l'identification des ambassadeurs locaux, ainsi que la Royal Society for the Conservation of Nature qui a contribué à l'organisation de l'atelier en Jordanie. Nous tenons également à remercier Maher Mahjoub, Güler Ekmekci, Panagiota Maragou, Krešimir Žganec, Zoran Marčić, Alain Jeudy, Alexa Dubreuil-Storer et Süreyya İsfendiyaroğlu pour leur traduction des termes clés et du Résumé dans les autres langues de la région étudiée. Enfin, nous souhaitons remercier lan Harrison pour son aide considérable dans le cadre de la conception et de l'élaboration de ce projet.

Ce projet a été financé par le Fonds de Partenariat pour les Écosystèmes critiques (projet 61452), la Fondation MAVA (projet 11-013) et l'Agence espagnole pour la coopération internationale pour le développement (AECID), et avec la contribution du projet Biofresh financé par la Commission européenne (projet 226874) et l'Organisme autonome des parcs nationaux espagnols (OAPN) du Ministère espagnol de l'Agriculture, de l'Alimentation et de l'Environnement. 
Termes clés dans les langues de la région étudiée

\begin{tabular}{|c|c|c|c|c|}
\hline & French & Arabic & Turkish & Greek \\
\hline Biodiversity & Biodiversité & التتوع البيولوجي & Biyolojik çeşitlilik (Biyoçeşitlilik) & Вıотоікıло́тпта \\
\hline Aquatic plants & Plantes aquatiques & 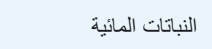 & Sucul bitkiler & 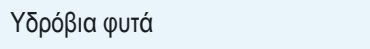 \\
\hline Freshwater fish & Poisson d'eau douce & 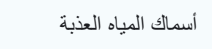 & Tatısu balıkları & 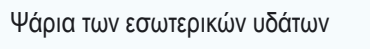 \\
\hline Molluscs & Mollusques & 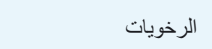 & Yumuşakcalar & Ma入ákıa \\
\hline $\begin{array}{l}\text { Odonata (dragonflies and } \\
\text { damselflies) }\end{array}$ & $\begin{array}{l}\text { Odonates (libellules et } \\
\text { demoiselles) }\end{array}$ & 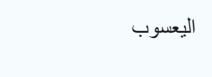 & $\begin{array}{l}\text { Yusufcuklar- Helikopter } \\
\text { böcekleri }\end{array}$ & 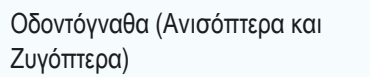 \\
\hline Shrimps & Crevettes & 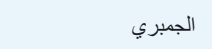 & Karidesler & 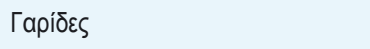 \\
\hline Crabs & Crabes & السرطانات & Yengeçler & Kaßoúpıa \\
\hline $\begin{array}{l}\text { IUCN Red List of } \\
\text { Threatened Species }\end{array}$ & $\begin{array}{l}\text { Liste rouge des espèces } \\
\text { menacées de l'UICN }\end{array}$ & 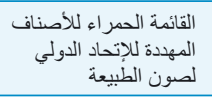 & $\begin{array}{l}\text { IUCN Tehdit altındaki türler } \\
\text { Kırmızı listesi }\end{array}$ & 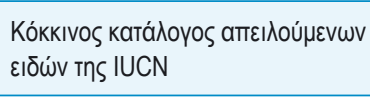 \\
\hline $\begin{array}{l}\text { Critically Endangered } \\
\text { (IUCN Red List Category) }\end{array}$ & $\begin{array}{l}\text { En danger critique (catégorie de } \\
\text { la Liste rouge de l'UICN) }\end{array}$ & 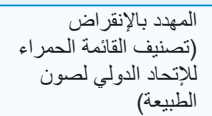 & $\begin{array}{l}\text { Kritik düzeyde tehlikede (IUCN } \\
\text { Kırmızı Liste Kategorisi) }\end{array}$ & 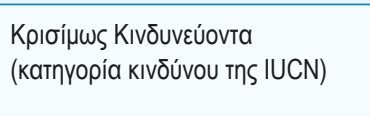 \\
\hline $\begin{array}{l}\text { Endangered } \\
\text { (IUCN Red List Category) }\end{array}$ & $\begin{array}{l}\text { En danger (catégorie de la Liste } \\
\text { rouge de l'UICN) }\end{array}$ & 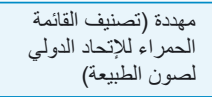 & $\begin{array}{l}\text { Tehlike altında (IUCN Kırmızı } \\
\text { Liste Kategorisi) }\end{array}$ & 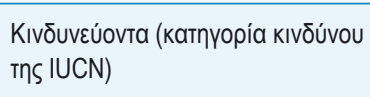 \\
\hline $\begin{array}{l}\text { Vulnerable } \\
\text { (IUCN Red List Category) }\end{array}$ & $\begin{array}{l}\text { Vulnérable (catégorie de la Liste } \\
\text { rouge de l'UICN) }\end{array}$ & 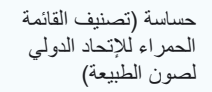 & $\begin{array}{l}\text { Duyarlı (IUCN Kırmızı Liste } \\
\text { Kategorisi) }\end{array}$ & 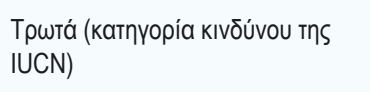 \\
\hline $\begin{array}{l}\text { Data Deficient } \\
\text { (IUCN Red List Category) }\end{array}$ & $\begin{array}{l}\text { Données insuffisantes (catégorie } \\
\text { de la Liste rouge de l'UICN) }\end{array}$ & القائمة الحمر اء للإتحناد (تصنيف & $\begin{array}{l}\text { Yetersiz Veri } \\
\text { (IUCN Kırmızı Liste Kategorisi) }\end{array}$ & 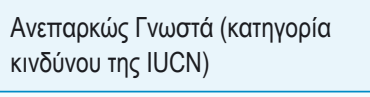 \\
\hline $\begin{array}{l}\text { Freshwater Key Biodiversity } \\
\text { Areas (KBA) }\end{array}$ & $\begin{array}{l}\text { Zones clés pour la biodiversité } \\
\text { (ZCB) d'eau douce }\end{array}$ & البيولوجي اللمياه العذبة اللنّة & Önemli Tatlısu Alanı (ÖTA) & 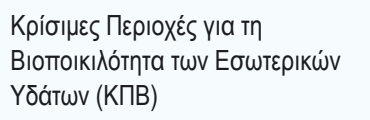 \\
\hline KBA Criteria & Critères de ZCB & للتنوع البيولوجير المواقع الهامة & ÖTA Kriterleri & 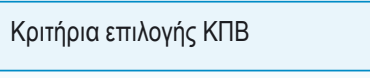 \\
\hline $\begin{array}{l}\text { KBA Criteria } \\
\text { Criterion 1: A site is } \\
\text { known or thought to hold a } \\
\text { significant number of one } \\
\text { or more globally threatened } \\
\text { species or other species of } \\
\text { conservation concern. }\end{array}$ & $\begin{array}{l}\text { Critères ZCB } \\
\text { Critère } 1 \text { : Le site abrite (a priori } \\
\text { ou effectivement) un nombre } \\
\text { significatif d'individus d'une ou de } \\
\text { plusieurs espèces menacées au } \\
\text { niveau mondial, ou d'autres } \\
\text { espèces d'intérêt pourlaconservation }\end{array}$ & 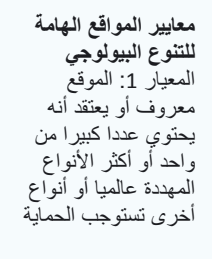 & $\begin{array}{l}\text { ÖTA Kriterleri } \\
\text { Kriter 1: Küresel ölçekte tehdit } \\
\text { altında olan bir veya birkaç } \\
\text { türün ve korumada öncelikli } \\
\text { diğer tür vey a türlerin önemli } \\
\text { sayıda bireyinin barındığı } \\
\text { bilinen ya da düşünülen alan }\end{array}$ & 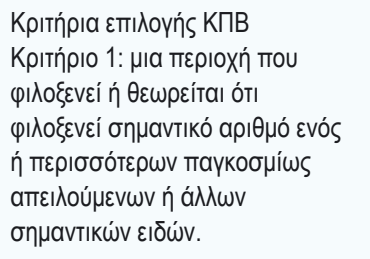 \\
\hline $\begin{array}{l}\text { KBA Criteria } \\
\text { Criterion 2: A site is } \\
\text { known or thought to hold } \\
\text { non-trivial numbers of } \\
\text { one or more species } \\
\text { (or infraspecific taxa } \\
\text { as appropriate) of } \\
\text { restricted range. }\end{array}$ & $\begin{array}{l}\text { Critères ZCB } \\
\text { Critère } 2 \text { : Le site abrite (a priori } \\
\text { ou effectivement) un nombre } \\
\text { d'individus non négligeable d'une } \\
\text { ou de plusieurs espèces (ou de } \\
\text { taxons infraspécifiques) ayant } \\
\text { une aire de répartition restreinte }\end{array}$ & 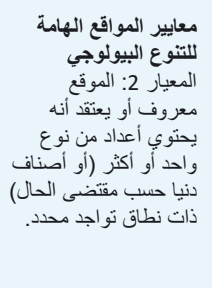 & $\begin{array}{l}\text { ÖTA Kriterleri } \\
\text { Kriter 2: Dar yayılışlı bir } \\
\text { veya birkaç türün (ya da } \\
\text { infraspesifik taksonun) } \\
\text { önemsiz sayılamayacak sayıda } \\
\text { bireyini barındırdığı bilinen ya } \\
\text { da düşünülen alan }\end{array}$ & 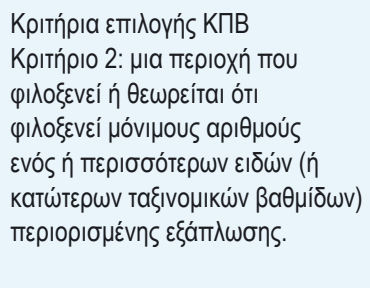 \\
\hline $\begin{array}{l}\text { KBA Criteria } \\
\text { Criterion 3: A site is known } \\
\text { or thought to hold a } \\
\text { significant component of the } \\
\text { group of species that are } \\
\text { confined to an appropriate } \\
\text { biogeographic unit or units. }\end{array}$ & $\begin{array}{l}\text { Critères ZCB } \\
\text { Critère 3: Le site abrite (a priori ou } \\
\text { effectivement) une composante } \\
\text { significative d'un groupe } \\
\text { d'espèces limitées à une ou } \\
\text { plusiers unités biogéographiques } \\
\text { appropriées }\end{array}$ & 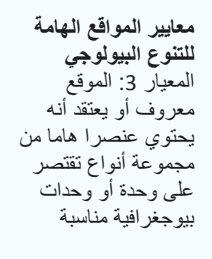 & $\begin{array}{l}\text { ÖTA Kriterleri } \\
\text { Kriter 3: } \\
\text { Belirli bir biyocoğrafik birim } \\
\text { veya birimlerde yaşayabilen } \\
\text { tür gruplarının önemli bir } \\
\text { bölümünü barındırdığı bilinen } \\
\text { ya da düşünülen alan }\end{array}$ & 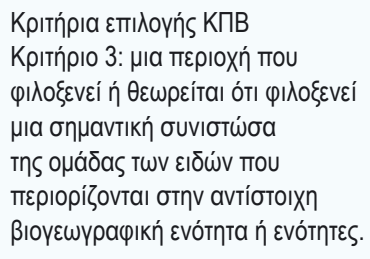 \\
\hline KBA Focal Areas & Zone focales d'une ZCB & الهامة للتنوع البيولوجيز للمواقع & ÖTA Odak Alanları & 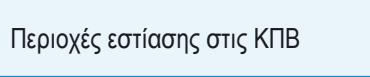 \\
\hline KBA Trigger species & Espèce de déclenchement de ZCB & 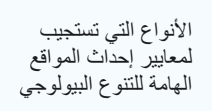 & ÖTA türleri & 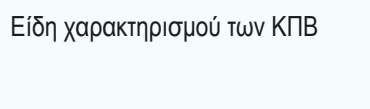 \\
\hline Conservation actions & Actions de conservation & 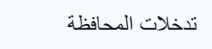 & & \\
\hline Threats & Menaces & 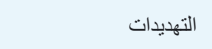 & Tehditler & 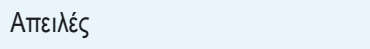 \\
\hline Stakeholders & Parties prenantes & الأطر اف المعنية & Paydaşlar & 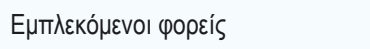 \\
\hline
\end{tabular}




\section{Résumé}

Le " hotspot » (point chaud) de la biodiversité du bassin méditerranéen est bien connu pour l'importance que sa biodiversité revêt à l'échelle mondiale. II est aujourd'hui confirmé que la biodiversité d'eau douce existant dans ce hotspot présente une diversité inhabituelle, abrite de nombreuses espèces endémiques aux cours d'eau, rivières, sources, zones humides et lacs de la région, et est fortement menacée, alors que l'importance de cette biodiversité n'était pas reconnue précédemment.

Les Zones clés pour la biodiversité (ZCB) sont des sites qui contribuent de manière significative à la persistance globale de la biodiversité. À partir des informations publiées concernant le statut de conservation et la répartition géographique des espèces (source : Liste rouge des espèces menacées de I'UICN), il a été identifié que $90 \%$ des 3894 sous-bassins versants fluviaux/lacustres étudiés étaient susceptibles de remplir les critères d'admission en tant que ZCB d'eau douce.

Les principales menaces pesant sur les espèces d'eau douce au sein du hotspot sont la gravité accrue des sécheresses, les altérations hydrologiques suite à la construction de barrages, le captage excessif des eaux souterraines et de surface, la pollution de l'eau et les espèces envahissantes. Les effets de ce type de menaces ont tendance à se répercuter rapidement au sein des bassins versants, à tel point que les actions de conservation localisées se limitant à des parties restreintes d'un bassin versant ne parviennent généralement pas à faire face à ces menaces. Pour cette raison, l'unité de gestion adaptée pour la plupart des ZCB d'eau douce est un sous-bassin ou un groupe de sousbassins reliés entre eux. Les principaux critères utilisés pour qu'un sous-bassin versant soit admis en tant que « ZCB proposée " ont été la présence d'espèces menacées ou ayant une aire de répartition restreinte, ou bien la présence d'une communauté d'espèces restreinte à une écorégion.

L'évaluation ultérieure de ces ZCB proposées, concernant les trois sous-régions (Balkans, Turquie \& Levant et Afrique du Nord) du hotspot éligible au financement du CEPF, a été réalisée dans le cadre de trois ateliers réunissant 39 parties prenantes et organisés à Jahorina (Bosnie-Herzégovine) pour les Balkans, à Azraq (Jordanie) pour la Turquie et le Levant, et à Marrakech (Maroc) pour l'Afrique du Nord. Cent soixante-sept ZCB d'eau douce, couvrant une superficie totale de $302557 \mathrm{~km}^{2}$, ont été confirmées en tant que ZCB d'eau douce valides. Sur ces 167 ZCB, 40 remplissent également les critères d'admission en tant que sites "Alliance for Zero Extinction » (Alliance pour une extinction zéro ou AZE), dans lesquels des actions de conservation immédiates sont requises pour qu'une espèce présente dans la ZCB ne s'éteigne pas à l'échelle mondiale dans un avenir proche. Toutes les ZCB proposées et validées peuvent à présent être visualisées par le public, en consultant le site Web de la base de données pour la biodiversité mondiale (WBDB, World Biodiversity DataBase) (www.birdlife.org/ datazone/freshwater).
Il a été constaté que le niveau actuel d'inclusion de ZCB d'eau douce validées au sein des aires protégées existantes ou des autres ZCB était extrêmement bas. Ainsi, il a été observé que $75 \%$ de la superficie totale couverte par ces ZCB se situait en dehors des limites géographiques des aires protégées existantes ou des autres ZCB, y compris 15 ZCB d'eau douce pour lesquelles il n'existe aucun chevauchement.

Dans le cadre de ce projet, les ZCB d'eau douce ont maintenant été identifiées, cartographiées et validées dans une grande partie du hotspot méditerranéen. À présent, il est important de faire prendre conscience de leur statut en tant que ZCB d'eau douce validées, et de mettre en place des programmes d'actions de conservation adaptées sur ces sites.

Les parties prenantes ont identifié 188 « Ambassadeurs locaux » [en anglais : Site Champions] potentiels parmi les personnes/organisations les mieux placées pour sensibiliser à l'existence des ZCB et aux difficultés rencontrées en raison des menaces pesant sur la biodiversité, et pour aider à mettre en œuvre les actions requises en vue de préserver ces sites dont l'importance est de portée mondiale.

Les recommandations spécifiques en matière d'actions de conservation sont principalement axées sur l'amélioration de la gestion hydrologique de ces ZCB, dont beaucoup sont actuellement ou potentiellement affectées par le captage excessif et le détournement de l'eau, la construction de barrages et les sécheresses. Les ZCB doivent être gérées de manière à veiller à ce que les flux environnementaux soient suffisants pour soutenir ces écosystèmes d'eau douce si fragiles, et elles doivent être établies dans le cadre d'une planification de la Gestion intégrée des bassins hydrographiques $(\mathrm{GIBH})$ à l'échelle des bassins versants, en tenant compte de la diversité des utilisations de l'eau selon les différents secteurs. II existe également des lacunes importantes en termes de connaissances sur la répartition de nombreuses espèces menacées figurant parmi les espèces spécifiques aux bassins et aux sites, et de nombreux pays n'ont pas encore d'inventaires de base concernant les écosystèmes de leurs eaux intérieures et les assemblages d'espèces. Beaucoup de nouvelles ZCB seront très probablement identifiées au fur et à mesure de l'avancement de ces inventaires de la biodiversité.

En conclusion, il est clair que le hotspot du bassin méditerranéen revêt une importance mondiale pour sa biodiversité d'eau douce. Cette biodiversité est fortement menacée, en grande partie à cause de la concurrence de la demande dans un contexte de diminution de l'offre en eau douce, et cette menace est exacerbée encore davantage par la gravité accrue des sécheresses survenant dans la région. Des espèces seront presque certainement perdues dans un avenir très proche si les recommandations formulées plus haut ne sont pas suivies et si des Ambassadeurs locaux ne se mobilisent pas pour sensibiliser à l'importance mondiale de ces ZCB d'eau douce. Des solutions existent mais la volonté de les faire adopter doit être encouragée. La plupart du temps, la situation des espèces d'eau douce confirme l'adage " loin des yeux, loin du cœur », c'est pourquoi il est fondamental de sensibiliser à leur présence, aux menaces 
pesant sur ces espèces et aux actions de conservation nécessaires, ceci afin de garantir la persistance de la biodiversité d'eau douce dans le hotspot méditerranéen.

\section{Executive summary}

The Mediterranean Basin Biodiversity Hotspot is well known for its globally important biodiversity. The freshwater biodiversity in the Hotspot, not previously widely recognized for its importance, is confirmed here to be unusually diverse and highly threatened, with many species endemic to individual rivers, streams, springs, wetlands and lakes across the region.

Key Biodiversity Areas (KBAs) are areas contributing significantly to the global persistence of biodiversity. Based on published information on species conservation status and distributions (source IUCN Red List of Threatened Species), $90 \%$ of the 3,894 river/lake sub-catchments considered were found likely to meet the criteria qualifying them as Freshwater KBAs.

The primary threats to freshwater species across the hotspot are increasing severity of droughts, hydrological alterations following construction of dams, over-abstraction of surface and ground waters, water pollution and invasive species. The impacts of these types of threat tend to spread rapidly throughout catchments such that localized conservation actions restricted to limited parts of a catchment will often fail to address these threats. For this reason the appropriate management unit for most freshwater KBAs is a subcatchment, or a group of connected sub-catchments. The main criteria employed for a sub-catchment to qualify as a 'proposed KBA' were the presence of threatened or restricted range species, or an ecoregion-restricted community of species.

Subsequent evaluation of these proposed KBAs for the three sub-regions of the Hotspot eligible for CEPF funding was conducted through three workshops involving 39 stakeholders in the Balkans (Jahorina, Bosnia and Herzegovina), Turkey and Levant (Azraq, Jordan), and northern Africa (Marrakesh, Morocco). One hundred and sixty-seven freshwater KBAs, covering a total area of $302,557 \mathrm{~km}^{2}$ were confirmed as valid freshwater KBAs. Of these, $40 \mathrm{KBAs}$ also meet the criteria qualifying them as Alliance for Zero Extinction (AZE) sites where immediate conservation actions are required if a species present in the KBA is not to become globally extinct in the near future. All proposed and validated KBAs are now publicly available for viewing on the World Biodiversity DataBase website (www.birdlife.org/datazone/freshwater).

The current level of inclusion of validated freshwater KBAs within existing protected areas or other KBAs was found to be extremely low. Seventy-five per cent of the total area of these KBAs was found to lie outside the boundaries of any pre-existing protected areas or other KBAs, including 15 freshwater KBAs for which there is no overlap at all.
Through this project freshwater KBAs have now been identified, mapped and validated throughout much of the Mediterranean Hotspot. It is now important to raise awareness of their status as validated freshwater KBAs and to develop plans for appropriate conservation actions at these sites. One hundred and eighty-eight potential Site Champions have been identified by stakeholders as individuals/organizations best placed to raise awareness of the existence of the KBAs and the issues faced with respect to threats to biodiversity, and to help implement the required actions to safeguard these globally important sites.

Specific recommendations for conservation actions are mainly focused on improving management of the hydrology of these KBAs, many of which are currently or potentially impacted by over-abstraction and diversion of water, construction of dams, and drought. KBAs need to be managed to ensure Environmental Flows are sufficient to support these fragile freshwater ecosystems and they should be implemented as part of catchment-wide Integrated River Basin Management planning which takes account of the wide range of uses of water across all sectors. There are also important knowledge gaps in site and basin-specific species distributions of many threatened species, and many countries do not yet have baseline inventories of their inland water ecosystems and species assemblages. It is very possible that many new KBAs will be discovered if these biodiversity inventories progress.

In conclusion, the Mediterranean Basin Hotspot is found to be globally important for its freshwater biodiversity. This biodiversity is highly threatened largely due to the conflicting demands upon a diminishing supply of fresh water which is further exacerbated by the increased severity of drought across the region. Unless the recommendations given above are followed and Site Champions are mobilized to raise awareness of these globally important freshwater KBAs, species will almost certainly be lost in the very near future. Solutions are available but the willingness to adopt them has to be encouraged. Freshwater species are most often out of sight and out of mind so raising awareness of their presence, the threats they face, and the necessary conservation actions are fundamental to the persistence of freshwater biodiversity in the Mediterranean Hotspot.

\section{Sažetak}

Područje Mediterana dobro je poznata „vruća točka” bioraznolikosti od globalne važnosti. Bioraznolikost slatkovodnih ekosustava Mediterana, čija važnost još uvijek nije široko prepoznata, u ovom istraživanju je potvrđena kao izuzetno velika, ali i jako ugrožena, s mnogim endemičnim vrstama koje nastanjuju pojedine rijeke, potoke, izvore, močvare i jezera ovog područja.

Na temelju objavljenih informacija o statusu ugroženosti vrsta i njihovoj rasprostranjenosti (izvor: Crvena lista IUCN-a), za 3513 od ukupno 3894 analiziranih riječnih/jezerskih pod- 
slivova, ustanovljeno je da najvjerojatnije sadrže vrste zbog kojih zadovoljavaju kriterije da in možemo kvalificirati kao ključna područja slatkovodne bioraznolikosti (engl. Freshwater Key Biodiversity Areas - KBAs), koja se definiraju kao područja koja značajno pridonose globalnom očuvanju bioraznolikosti.

Primarne prijetnje slatkovodnim vrstama u području Mediterana, „vruće točke” bioraznolikosti, su onečišćenje voda, porast jačine suša, hidrološke promjene zbog izgradnje brana, pretjerano crpljenje površinskih i podzemnih voda te invazivne vrste. Učinci ovakvih tipova prijetnji imaju tendenciju brzog širenja slivom tako da lokalizirane mjere zaštite koje su ograničene na pojedine dijelove sliva najčešće ne mogu ukloniti ove prijetnje. Iz tog je razloga za većinu slatkovodnih KBA-ova odgovarajuća jedinica upravljanja podsliv ili grupa povezanih podslivova. Glavni kriterij da se podsliv kvalificira kao predloženi KBA bio je prisutnost ugroženih vrsta ili onih s ograničenim područjem rasprostranjenosti, ili vrsta koje predstavljaju reprezentativan skup vrsta pojedine slatkovodne ekoregije.

Procjena predloženih KBA-ova za tri podregije „vruće točke" pogodnih za financiranje iz fonda CEPF-a provedena je kroz tri radionice uključujući 39 dionike na području Balkana (Jahorina, Bosna i Hercegovina), Turska i Levant (Azrak, Jordan), i sjeverna Afrika (Marakeš, Moroko). Ukupno je 167 slatkovodnih KBA-ova, koji pokrivaju ukupno $302.557 \mathrm{~km}^{2}$, potvrđeno da zadovoljavaju kriterije koji in kvalificiraju za slatkovodne KBA-ove. Od toga, 40 KBA-ova također ispunjava kriterije koji in kvalificiraju kao područja "Saveza za nultu stopu izumiranja” (engl. Alliance for Zero Extinction - AZE) u kojima su potrebne žurne konzervacijske akcije kako vrste prisutne u KBA-u ne bi globalno izumrle $u$ bliskoj budućnosti. Svi predloženi i vrednovani KBA-ovi su sada javno dostupni za pregled na internetskim stranicama svjetske baze bioraznolikosti, World Biodiversity DataBase (www.birdlife.org/datazone/freshwater).

Trenutna razina uključivanja potvrđenih slatkovodnih KBAova uključenih u postojeća zaštićena područja i druge (kopnene) KBA-ove je izrazito niska. $75 \%$ slatkovodnih KBA-ova leži izvan granica postojećih zaštićenih područja i drugih KBA-ova, uključujući i 15 slatkovodnih KBA-ova s kojima uopće nije bilo preklapanja s zaštićenim područjima ili drugim KBA-ovima.

Kroz ovaj projekt slatkovodni KBA-ovi su identificirani, kartirani i potvrđeni za većinu „vruće točke“ Mediterana. Sada je potrebno podići svijest o njihovom statusu kao potvrđenim slatkovodnim KBA-ovima i razviti planove za odgovarajuće konzervacijske akcije na ovim područjima. 188 Dionici su identificirali potencijalne Pobornike područja kao pojedince ili organizacije koji mogu podići svijest o postojanju KBA-ova i problemima koji im prijete $s$ aspekta bioraznolikosti te koji mogu pomoći provesti potrebne mjere kojima bi se očuvala ova globalno važna područja.

Naročite preporuke za konzervacijske akcije pretežno su usmjerene na unaprjeđenje upravljanjem hidrologijom ovih KBA-ova od kojih su mnogi trenutno ili potencijalno pogođeni gradnjom brana, pretjeranim korištenjem vode, skretanjem vodotokova i sušama. KBA-ovima treba upravljati tako da se osiguraju ekološki prihvatljivi protoci koji su dovoljni za održavanje ovih krhkih slatkovodnih ekosistema i trebali bi biti ostvareni kao dio plana upravljanja čitavog sliva (Integrated River Basin Management) koji uzima u obzir ukupno korištenje vode u svim sektorima.

Zaključno, Mediteranska „vruća točka“ prepoznata je kao globalno važna zbog svoje slatkovodne bioraznolikosti. Ova bioraznolikost je izrazito ugrožena zbog potreba za korištenjem slatke vode čija količina se smanjuje, a koja je dodatno pojačana sve jačim sušama diljem regije. Ako se preporuke iznesene ranije ne provedu u djelo i ako se ne aktiviraju Pobornici područja koji će podići stanje svijesti o ovim globalno važnim slatkovodnim KBA-ovima, ugrožene vrste koje u njima dolaze će sigurno izumrijeti u vrlo bliskoj budućnosti. Postoje rješenja, ali spremnost da ih se usvoji treba poticati. Slatkovodne su vrste često zaboravljene jer su nedostupne i nevidljive većini pa su podizanje razine svijesti o njihovom postojanju, o prijetnjama koje ih ugrožavaju i potrebnim konzervacijskim akcijama vrlo važan prvi korak.

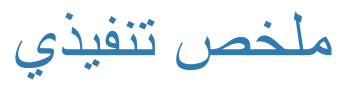

النقطة الساخنة للتنوع البيولوجي بحوض البحر الأبيض المتوسط

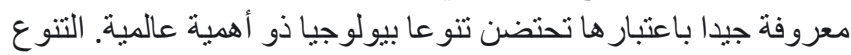

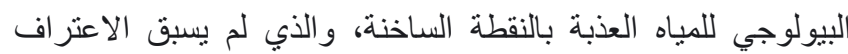

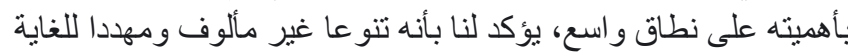

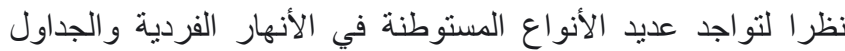
و الينابيع و الأر اضي الرطبة و البحير ات في جميع أنحاء المنطقة.

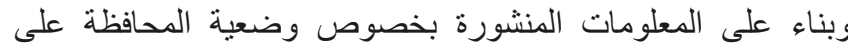

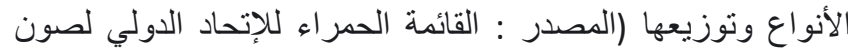

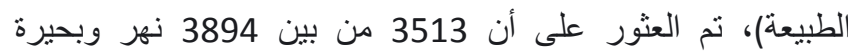
ومستجمعات مياه فرعية تحتوي على الأنواع التي تفي بمعايير تأهيلها

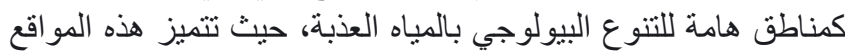

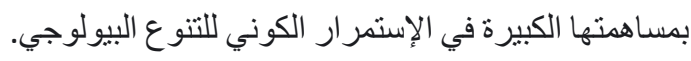

إن التهديدات الرئيسية للأنواع المتو اجدة بالمياه العذبة في جميع أنحاء

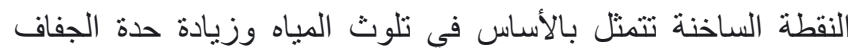

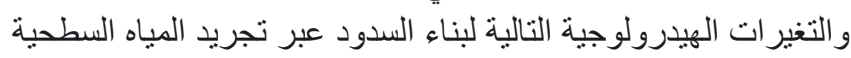

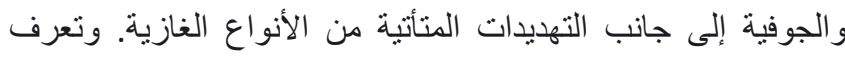
تأثير ات هذه التهديدات انتشار التريعا في جميع أنحاء مستجمعات المياه

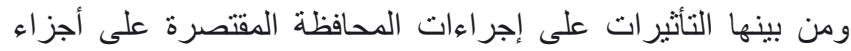

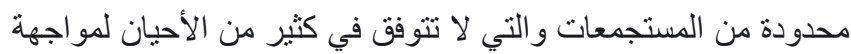

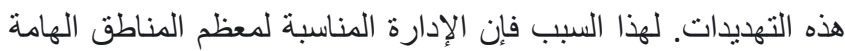

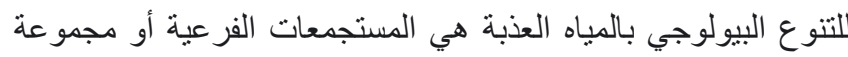

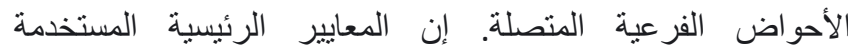

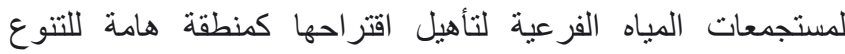


البيولوجي هي بالأساس وجود طائفة أنواع مهددة أو مقيدة، أو أنواع ممثلة لمجموع المياه العذبة بالمنطقة الإيكولوجية.

وقد تم إجر اء تقييم للمناطق الهامة للتنوع البيولوجي المقترحة للمناطق

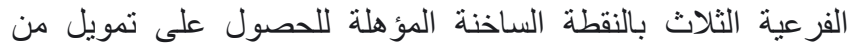

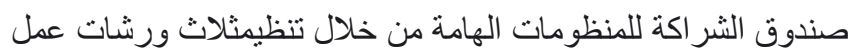

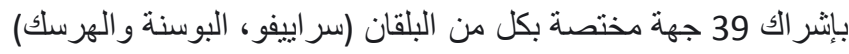

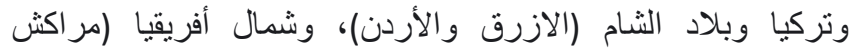

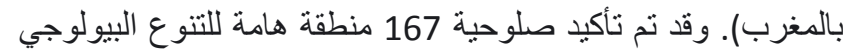
بالمياه العذبة، و التي تغطي مساحة إجمالية تبلغ بينها 40 منطقة هامة للتنوع البيولوجي تستجيب كذلك لمعايير تأهيلها لـ" التحالف من أجل مو اقع بدون انقر اضه" مما يتطلب إجر اءات فورية

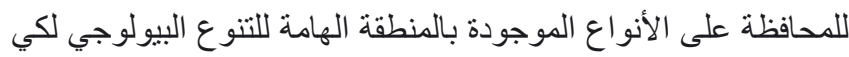

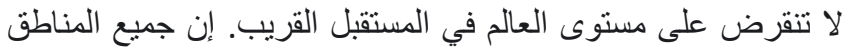

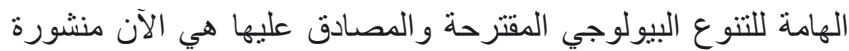

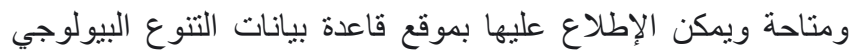

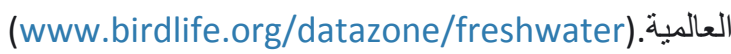

إن المستوى الحالي لإدراج المناطق الهامة للتنوع البيولوجي بالمياه

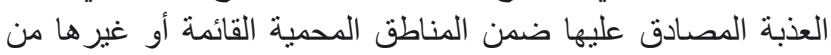

المناطق الهامة للتنوع البيولوجي يعتبر منخفض للغاية. حيث أن خمسة

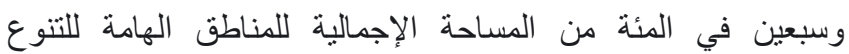

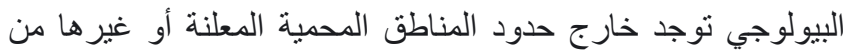
المناطق الهامة، بما في ذلك 15 منطقة هامة للتنوع البيولوجي بالمئودياه العذبة لا يوجد بها أي تداخل على الإطلاق.

وقد تم عبر هذا المشروع تحديد المناطق الهامة للتنوع البيولوجي دواني

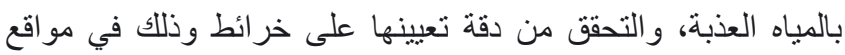

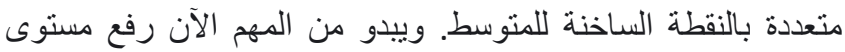
الوعي بخصوص وضعهم الحالي إبان المصادقة على المناطق الهامة لإنة للتنوع البيولوجي بالمياه العذبة إلى جانب وضع وضع خطط لإجراءات محافظة ملائمة في هذه المو اقع. وقد تم تحديد 188 موقع رئيسي محتمل الفي جانب

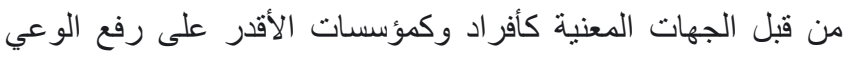

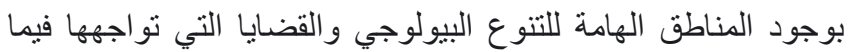
يتعلق بالتهديدات على التنوع البيولوجي و المساعدة في تنفيذ الإجر اءات التولئ اللازمة لحماية هذه المو اقع الهامة عالميات التوليا.

وتتركز التوصيات الخصوصية لإجر اءات المحافظة أساسا على تحسين الإدارة الهيدرولوجية للمناطق الهامة للتتوع البيولوجي، والتي تأثرت أترات كثير نتيجة بناء السدود، عبر تجريد وتحويل المياه، وكذلك الجفاف.

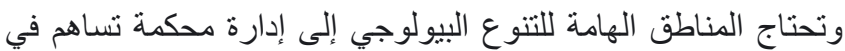

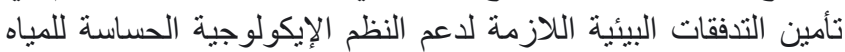

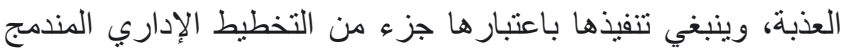

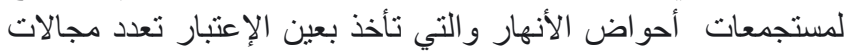
استخدامات المياه في جميع القطاعات.

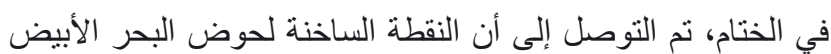

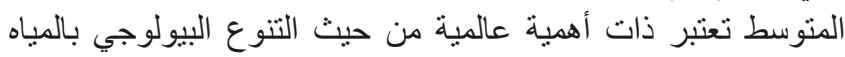

العذبة. ويعرف هذا التنوع البيولوجي تهديدا كبيرا بسبب المطالب المتضاربة على المياه العذبة الذي ازدادت حدتها في ضل ضل تفاقم ظاهرة

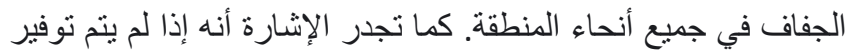

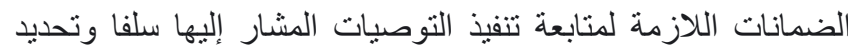

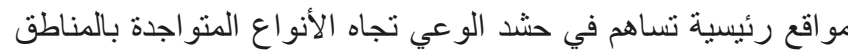

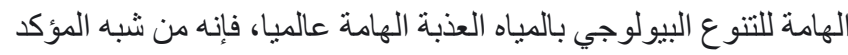

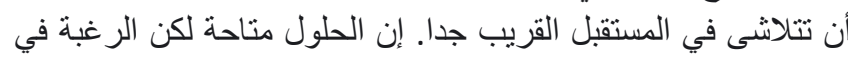

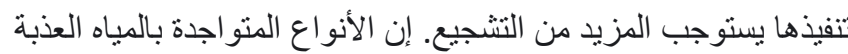

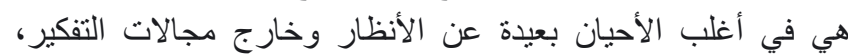

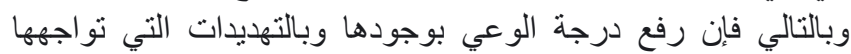

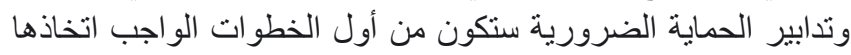

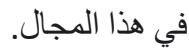

\section{Genişletilmiş Özet}

Akdeniz Havzası Biyoçeşitlilik sıcak noktası küresel öneme sahip biyoçeşitliliğiyle tanınır. Sıcak noktanın daha önce önemi geniş kitlelerce bilinmeyen, bölgede yer alan bir çok nehre, akarsuya, kaynağa, sulak alana ve göle endemik türlere sahip tatlısu biyoçeşitliliğinin olağanüstü çeşitli, son derece tehlike altında olduğu burada teyid edilmiştir.

Önemli Doğa Alanları (ÖDA’lar) biyolojikçeşitliliğin küresel devamlılığına hatrı sayılır destek sağlayan alanlardır. Tür durum ve dağılımlarına dair basılı kaynaklara (kaynak IUCN Nesli Tehlike Altında Türler Kırmızı Listesi) değerlendirilen 3,894 nehir/göl althavzasından \%90'ının kriteleri sağlayarak Önemli Tatısu Alanı olduğu tespit edilmiştir.

Sıcak noktada tatlısu türlerini tehdit eden başlıca tehditler, su kirliliği, kurakllığın gittikçe daha sertleşmesi, Baraj inşaatları, yeraltı ve yüzey su kaynaklarının aşırı kullanımı gibi şekillerde suyun akışına müdahale edilmesi ve istilacı türlerdir. Bu tip tehditlerin etkileri havzalar boyunca hızlıca yayılma eğilimindedir, öyle ki, havzaların belli kısımlarıyla sınırlandırılan bölgesel koruma eylemleri çoğu zaman bu tehditleri ele almakta yetersiz kalmaktır. Bu nedenle, birçok tatlısu ÖTA'sı için uygun yönetim birimi bir alt-havza veya birbirine bağlı bir alt-havzalar grubudur. Bir alt-havzanın "aday ÖTA" olarak sınıflandırılması için gereken başlıca kriter, tehdit altında veya daryayılışlı bir türün veya bir tatlısu ekolojik bölgesine özgü tür topluluğunu temsil eden türlerin varlığıdır.

Sıcak noktanın üç alt-bölgesi için önerilen ÖTA'ların aşağıda yer alan değerlendirmeleri, CEPF fonu ile destelenerek, Balkanlardaki (Saraybosna, Bosna Hersek), Türkiye ve Doğu Akdeniz'deki (Azrak, Ürdün) ve Kuzey Afrika'daki (Marakeş ve Fas) 39 paydaşın katılımıyla gerçekleştirilen üç çalıştay ile yürütülmüştür. Toplam 302.557 km² alan kapsayan 167 tatlısu ÖTA'sı, onaylanmış ÖTA'lar olarak gerçelilik kazanmıştır. Bu alanlardan 40 tanesi kriteleri karşılayarak Sıfır Yok Oluş Alanı (Alliance for Zero Extinction-AZE) sınıflandırıımıştır. Sıfır Yok Oluş Alanları, Önemli Doğa Alanında bulunan bir türün yakın 
gelecekte küresel ölçekte yok olmaması için acil koruma çalışmalarına intiyaç duyulan alanlardır. Günümüzde tüm önerilen ve geçerli. Bütün aday ve onaylanmış ÖDA'lar Dünya Biyoçeşitlilik Veri Tabanı (World Biodiversity DataBase) (www.birdlife.org/datazone/freshwater) web sitesinde halkın kullanımına sunulmuş ve yayınlanmaktadır.

Onaylanmış ÖTA'ların hal,hazırda var olan korunan alanlarla veya diğer ÖDA'larla örtüşme seviyesinin son derece sınırlı olduğu görülmektedir. Hiçbir şekilde bir alanla örtüşmeyen 15 ÖTA da dahil olmak üzere bu ÖTA'ların toplam alanının $\% 75$ 'inin hali hazırda yer alan herhangi bir korunan alan veya ÖDA sınırının dışında yer aldığı görülmektedir.

Bu proje ile Akdeniz Sıcak Noktasında yer alan ÖTA'lar, tanımlanmış haritalanmış ve onaylanmıştır. Artık, bu alanların uzmanlarca onaylanmış ÖTAlar olarak tanıtılması ve bu alanlarda intiyaç duyulan koruma çalışmaları için gerekli planların geliştirilmesi önem arz etmektedir. 188 potansiyel Alan Şampiyonu (Site Champions) paydaşlar tarafından ÖDA'ların varlığına yönelik farkındalığın arttırılmasında en iyi konumda olan ve biyolojikçeşitliliğe karşı tehditlerle mücadele eden ve bu küresel öneme sahip alanların korunması için gerekli faaliyetleri yürüten kişiler/kuruluşlar olarak belirlenmiştir.

Koruma çalışmalarına yönelik spesifik tavsiyeler çoğunlukla alandaki su yönetiminin iyileştirilmesine odaklanmaktadır, ÖDA'ların birçoğu yeraltı suyunun aşırı kullanımı ve barajlarla suyun akış yönünün değiştirilmesi ve kuraklık gibi tehditlerden hali hazırda ya da potasiyel olarak etkilenmektedir. ÖDA'lar can suyunun güvence altına alındığı bu hassas ekosistemleri destekleyecek şekilde yönetilmeli ve bunlar havza ölçeğinde her sektörün geniş yelpazedeki su kullanımı ihtiyacını gözönünde bulunduran bütünleşik dere havzası yönetimi planlamasıyla uygulanmalıdır. Aynı zamanda bir çok nesli tehlike altında türün alan ve havzadaki dağılımlarına dair bilgi açıkları da mevcuttur ve bölgede bir çok ülkede içsu ekosistemlerinin temel tür envanteri ve tür toplulukları belirlenmiş değildir. Bu tip envanterler gerçekleştirildikçe yeni ÖDA'ların keifedilmesi de mümkündür.

Son olarak, Akdeniz sıcak noktasının içsulardaki çeşitlilik açısından küresel öneme sahip olduğu bulunmuştur. Bu biyoçeşitlilik büyük oranda bölgede şiddeti sürekli artan kuraklıktan da zarar gören ve her geçen gün azalan tatısu kaynaklarının temini konusunda ortaya çıkan yönetsel taleplerin yolaçtığı çıkan çıkar çatışmalarından dolayı yüksek tehdit altındadır. Yukarıda dile getirilen tavsiyeler gözönünde bulundurulmaz ve Alan Şampiyonları bu küresel ÖTA'larla ilgili farkındalığı arttırmak üzere harekete geçirilmezse, gelecekte hiç süphesizki türler kaybolacaktır. Hali hazırda çözüm ortadadır ve bunların hayata geçirilmesi özendirilmelidir. Tatlısu türleri çoğunlukla kişinin gözü önünde veya aklında bulunmayan türlerdir dolayısıyla Akdeniz Sıcak Noktasında bu türlerin varlığı, karşı karşıya kaldıkları tehditler konusunda farkındalık oluşturulması ve içsu biyolojik çeşitliliğin dayanma gücünü arıtaracak koruma uygulamalarının hayata geçirilmesi elzemdir. 


\section{Contexte}

\section{Le statut de la biodiversité d'eau douce dans le bassin méditerranéen}

Les écosystèmes d'eau douce occupent moins de $1 \%$ de la planète, et pourtant ils font partie des écosystèmes les plus variés et les plus menacés au monde (Strayer et Dudgeon 2010). Depuis des centaines d'années, les êtres humains drainent, remblaient et transforment les zones humides intérieures, à un point tel que la perte de ces écosystèmes au $X X^{\complement}$ siècle a été estimée à 69-75\% (Davidson 2014). Il existe peu d'informations sur les zones humides inondées de manière éphémère ou par intermittence, comme dans le cas des prairies inondables et des zones arides et semiarides à faible dépression. II n'existe pas non plus d'évaluation mondiale de l'état de santé des zones humides existant encore (Davidson 2014), même s'il est probable qu'un grand nombre d'entre elles soient aujourd'hui fortement dégradées. Étant donné qu'un dixième de toutes les espèces animales vivent en eau douce (Poff et al. 2012), y compris près de la moitié des espèces de poissons connues au niveau mondial (Carrizo et al. 2013), cette perte considérable d'habitat est une préoccupation importante pour l'atteinte des objectifs visant à mettre un terme à l'extinction courante des espèces. Plus de $29 \%$ des 25007 espèces d'eau douce évaluées dans la Liste rouge des espèces menacées de I'UICN" (" Liste rouge de I'UICN") (www.iucnredlist.org) sont menacées d'extinction au niveau mondial (UICN 2013). Cette proportion risque d'augmenter compte tenu du nombre élevé d'espèces d'eau douce appartenant à la catégorie "Données insuffisantes » $(n=5761)$ de la Liste rouge (UICN 2013) et des nombreuses espèces restant à évaluer (Poff et al. 2012).

L'une des plus grandes menaces pesant sur les espèces d'eau douce est donc la perte ou la dégradation des habitats (Collen et al. 2014 ; Garcia-Moreno et al. 2014), et le bassin méditerranéen n'échappe pas à la règle. La situation de la Méditerranée à l'égard des écosystèmes d'eau douce est particulièrement préoccupante en raison de la grande diversité des espèces endémiques soumises à une pression grandissante due au captage excessif de l'eau, à la propagation des espèces envahissantes et à la pollution. Les précédentes évaluations ont indiqué que plus de la moitié des poissons d'eau douce endémiques de la région, plus d'un tiers de tous les crabes et écrevisses, et près d'un tiers des amphibiens sont menacés (Cuttelod et al. 2008). Un cinquième de toutes les libellules méditerranéennes (Riservato et al. 2009 ; Samraoui et al. 2010) sont aussi menacées et la situation des mollusques d'eau douce méditerranéens est peu encourageante avec près de la moitié de toutes les espèces menacées en Europe (Cuttelod et al. 2011). Par conséquent, la protection des habitats par l'intermédiaire d'actions de conservation à l'échelle des sites, tels que les aires protégées, peut représenter un outil puissant pour contribuer à empêcher la perte d'espèces.

Les Zones clés pour la biodiversité (ZCB), dont l'importance est considérable au niveau mondial pour favoriser la persistance de la biodiversité (Eken et al. 2004 ; Langhammer et al. 2007), peuvent guider la sélection de nouvelles aires protégées ou l'élargissement des réseaux de sites existants. La science nous indique que les aires protégées n'ont pas toujours été bien positionnées stratégiquement et qu'elles risquent donc de ne pas fournir la meilleure protection pour la biodiversité qu'elles ont vocation à préserver ; ceci est particulièrement vrai dans le cas de la biodiversité d'eau douce (par exemple, Darwall et al. 2011). Initialement créé pour les oiseaux (ZICO, Zones importantes pour la conservation des oiseaux (Grimmett et Jones 1989)) et les plantes (ZIP, Zones importantes pour les plantes (Anderson 2002)), un ensemble de seuils et de critères normalisés a été développé pour identifier les ZCB de manière justifiable et transparente.

Les critères utilisés pour identifier les ZCB reposent sur la vulnérabilité et le caractère irremplaçable. Dans ce contexte, la vulnérabilité fait référence à la probabilité de perte progressive d'une espèce vivant au sein d'un site, et le caractère irremplaçable renvoie aux possibilités existant géographiquement pour la conservation d'une espèce particulière (Langhammer et al. 2007). La Liste rouge de l'UICN fournit les informations requises concernant les espèces, en vue de déterminer la vulnérabilité et le caractère irremplaçable de ces espèces et donc d'identifier les ZCB. Le raisonnement appliqué et les critères d'identification des ZCB (Eken et al 2004 ; Langhammer et al. 2007) sont le prolongement des concepts originaux relatifs aux Zones importantes pour la conservation des oiseaux (par exemple, Grimmett et Jones 1989). Toutefois, les méthodes utilisées pour identifier les ZCB concernant un grand nombre de groupes taxonomiques ne sont pas toujours homogènes en termes de critères de sélection ; c'est pourquoi les membres de I'UICN ont demandé à cette dernière de diriger un processus représenté au niveau mondial afin de rationaliser les procédures de ZCB (UICN 2014). Cette consultation, qui devrait prendre fin en 2014, a déjà permis d'accroître considérablement la visibilité de cet outil important.

Les ZCB d'eau douce doivent encore être identifiées pour la majorité des régions du globe, laissant actuellement peu d'occasions aux responsables de la conservation et du développement de tenir compte de la biodiversité d'eau douce dans le cadre du processus de planification (Darwall et al. 2011). En outre, il est urgent d'identifier les sites « Alliance for Zero Extinction » (AZE) d'eau douce (Ricketts et al. 2005), ces sites AZE représentant un important sous-ensemble des ZCB, abritant les dernières ou les seules populations d'espèces menacées au niveau mondial. En Europe, un seul site AZE d'eau douce a été identifié à ce jour ; il s'agit d'un site d'amphibiens abritant l'espèce Calotriton arnoldi (En danger critique) dans le parc naturel d'El Montseny en Espagne (Carranza et Amat 2005).

\section{Les ZCB en tant qu'outil de conservation}

L'identification des ZCB fournit des informations fondamentales pour éclairer différents types de décisions et d'utilisateurs finaux, notamment :

- en apportant un éclairage pour la sélection des sites à protéger, en vertu de la législation nationale et internationale.

- en mettant en œuvre et en surveillant les objectifs de biodiversité au niveau mondial, dans le cadre des accords 
environnementaux multilatéraux (par exemple, l'objectif d'Aichi $n^{\circ} 11$ de la Convention sur la diversité biologique).

- en aidant les secteurs privé et financier à gérer leurs risques environnementaux en termes d'impact sur la biodiversité.

- en orientant les investissements en faveur de la conservation, réalisés par les donateurs (Fonds de partenariat pour les écosystèmes critiques (CEPF), Fonds pour l'environnement mondial (FEM), etc.).

- en renforçant les actions de conservation sur le terrain, menées par les organisations non gouvernementales locales ou internationales.

\section{Objectifs du projet}

À la lumière de la situation décrite plus haut, le CEPF et la Fondation MAVA ont financé I'UICN en 2012 pour rechercher les informations manquantes dans le cadre des évaluations de la Liste rouge relatives aux espèces d'eau douce dans le hotspot du bassin méditerranéen (Figure 1), et travailler avec les parties prenantes concernées afin d'identifier et de valider les Zones clés pour la biodiversité dans le sud et l'est de la Méditerranée (voir Figure 1 pour visualiser la région visée par le projet). La vocation de ce projet a été de fournir les ressources essentielles à la prise de décisions en matière de conservation et de gestion durable de la biodiversité d'eau douce dans le hotspot de la biodiversité du bassin méditerranéen.

Ce projet a eu pour objectif, dans un premier temps, de combler le déficit d'informations sur la biodiversité d'eau douce dans les zones orientales du bassin, puis d'exploiter ces informations, en association avec les résultats de précédentes évaluations de la biodiversité, pour identifier les ZCB dans une grande partie du bassin. Ceci a pu être mené à bien de la manière suivante : (i) consolidation de données sur la répartition, l'abondance, l'écologie et l'utilisation par les êtres humains, pour plusieurs groupes d'espèces représentant des indicateurs fiables concernant la structure biologique et le fonctionnement des écosystèmes d'eau douce dans la région est de la Méditerranée ; (ii) évaluation du risque d'extinction pour ces espèces en fonction des Critères de la Liste rouge de l'UICN ; (iii) cartographie des caractéristiques géographiques de la richesse des espèces, de l'endémisme et des menaces actuelles ou imminentes pour les espèces (Smith et al. 2014) ; (iv) identification des sous-bassins versants fluviaux/lacustres abritant des espèces et remplissant a priori les critères de ZCB pour l'ensemble de la région du hotspot de la biodiversité du bassin méditerranéen ; (v) validation (après consultation auprès des parties prenantes) des sous-bassins versants qui remplissent effectivement les critères de ZCB en tant que ZCB dans les régions couvertes par le financement du CEPF ; et (vi) mise à disposition, librement et largement, des données et résultats consolidés issus des analyses, à l'attention des développeurs et des professionnels de la conservation.

Comme indiqué plus haut, l'importance et l'urgence de ce travail sont manifestes, que ce soit en raison de la forte concentration d'espèces vivant dans le bassin méditerranéen, des services écosystémiques fournis aux communautés humaines par ces eaux douces et leur biodiversité, ou bien des menaces de plus en plus nombreuses pesant sur ces écosystèmes.

Figure 1. Les limites sous-régionales du projet au sein du hotspot du bassin méditerranéen.

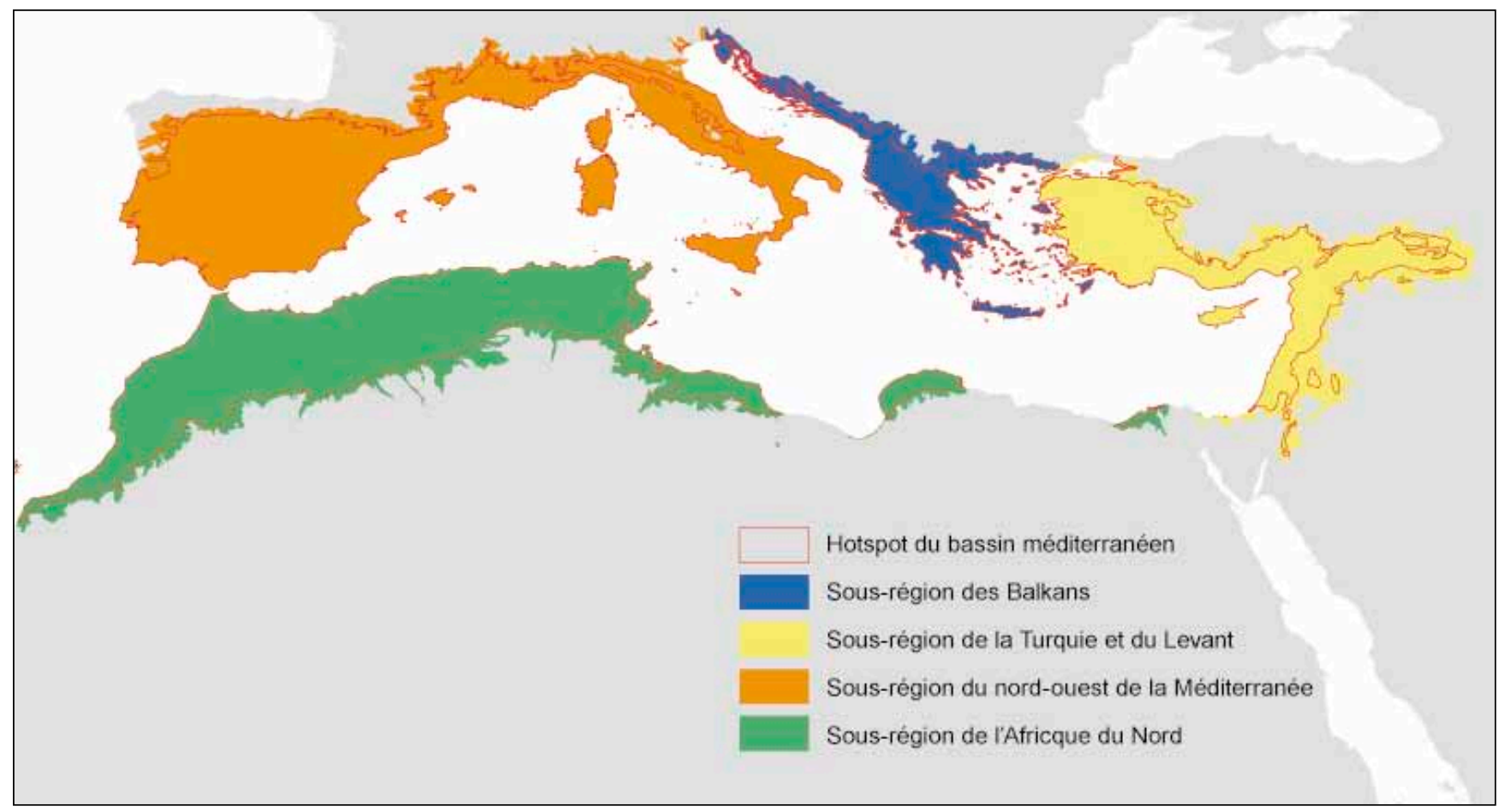




\section{Critères d'identification des Zones clés pour la biodiversité (ZCB) d'eau douce}

Comme indiqué plus haut, une consultation menée par l'UICN est organisée continuellement pour rationaliser les nombreux ensembles de critères et de seuils existants, utilisés afin d'identifier les ZCB pour différents groupes taxonomiques. Dans le cadre de ce projet, nous avons appliqué la seule méthodologie publiée pour l'identification et la délimitation des ZCB d'eau douce (Holland et al. 2012), qui a été créée conformément à la méthodologie générale dédiée aux ZCB. Les critères et les seuils utilisés sont résumés dans le Tableau 1. Pour plus de précisions sur les critères, les seuils et les méthodes utilisés, voir Holland et al. (2012). L'un des grands avantages de ces critères est le fait qu'ils permettent également l'identification de plans d'eau de petite taille ou même temporaires, qui sont généralement omis par les décideurs alors qu'ils restent importants pour la biodiversité d'eau douce.

L'identification et la validation des ZCB ont fait l'objet d'un processus en deux étapes :

Étape 1. Identification des ZCB proposées : zones définies comme étant l'ensemble des sous-bassins versants fluviaux/lacustres abritant des espèces remplissant les critères de ZCB.

Les informations relatives aux groupes taxonomiques suivants ont été consolidées pour identifier l'ensemble des sous-bassins versants fluviaux/lacustres du hotspot méditerranéen abritant les espèces respectant les seuils des critères de ZCB $n^{0} 1,2$, ou 3 (voir Tableau 1) : i) poissons d'eau douce ; ii) mollusques d'eau douce ; iii) libellules et demoiselles (odonates) ; et iv) plantes aquatiques. Les espèces remplissant les critères de ZCB sont définies en tant qu'espèces de déclenchement de ZCB. La présence d'espèces supplémentaires qui appartiennent

Tableau 1. Critères actuellement utilisés pour identifier une ZCB d'eau douce (adaptés de Holland et al. 2012).

Critère 1 : Le site abrite (a priori ou effectivement) un nombre significatif d'individus d'une ou de plusieurs espèces menacées au niveau mondial, ou d'autres espèces d'intérêt pour la conservation.

Seuil : La présence d'une ou de plusieurs espèces CR, EN ou VU déclenchera l'admission du site en tant que ZCB d'eau douce potentielle.

Critère 2 : Le site abrite (a priori ou effectivement) un nombre d'individus non négligeable d'une ou de plusieurs espèces (ou de taxons infraspécifiques) ayant une aire de répartition restreinte.

Seuil : Une valeur seuil de $20000 \mathrm{~km}^{2}$ a été appliquée aux poissons, aux plantes et aux mollusques, et une valeur seuil de $50000 \mathrm{~km}^{2}$ a été appliquée aux odonates.

Critère 3 : Le site abrite (a priori ou effectivement) une composante significative d'un groupe d'espèces limitées à une ou plusieurs unités biogéographiques appropriées.

Seuil : Pour déclencher l'admission, au moins $25 \%$ des espèces totales issues d'un groupe taxonomique spécifique doivent être restreintes à l'écorégion d'eau douce dans laquelle le bassin versant est situé. à d'autres taxons dépendant de l'eau douce a aussi été notée lorsque les informations étaient disponibles.

Les sous-bassins versants fluviaux/lacustres ont été délimités à partir de la couche de données spatiales "HydroBASINS ", développée récemment (Lehner et Gill 2013) (http://hydrosheds. org/page/hydrobasins) (Figure 2). Cette délimitation des bassins versants au niveau mondial a été personnalisée par I'UICN en modifiant les données HydroSHEDS existantes (Leyner et al. 2008) (http://hydrosheds.org) pour y inclure à présent les polygones relatifs aux lacs, provenant de la base de données GLWD (Global Lakes and Wetlands Database) consacrée aux lacs et zones humides mondiales (GLWD ; Lehner et Doll 2004). L'outil HydroBASINS est un cadre hydrologique normalisé mondial, délimitant les bassins versants selon 12 résolutions et incluant des informations sur la connectivité du réseau. Concernant la délimitation des ZCB, nous avons commencé par les bassins versants délimités selon la résolution HydroBASINS de niveau 8. La résolution HydroBASINS de niveau 8 en Europe couvre une superficie moyenne de $600 \mathrm{~km}^{2}$.

Cette première étape du processus a surtout été une analyse documentaire des données consolidées dans le cadre de la série de rapports publiés sur les évaluations de la Liste rouge de I'UICN pour la région. Ces ensembles de données comprennent les informations nécessaires sur la répartition des espèces (fichiers de formes numériques "Shapefile ») et sur leur risque d'extinction selon les Catégories de la Liste rouge de l'UICN, telles que publiées dans la Liste rouge de l'UICN. Les principales sources d'information sont notamment :

- La base de données en ligne relative à la Liste rouge des espèces menacées de I'UICN (www.iucnredlist.org)

- Bilz, M., Kell, S.P., Maxted, N. et Lansdown, R.V. (2011). European Red List of Vascular Plants. Luxembourg : Office des publications de l'Union européenne.

- Cuttelod, A., Seddon, M. et Neubert, E. (2011). European Red List of Non-marine Molluscs. Luxembourg : Office des publications de l'Union européenne.

- Freyhof, J. et Brooks, E. (2011). European Red List of Freshwater Fishes. Luxembourg : Office des publications de l'Union européenne.

- García, N., Cuttelod, A. et Abdul Malak, D. (eds.) (2010). The Status and Distribution of Freshwater Biodiversity in Northern Africa. Gland (Suisse), Cambridge (Royaume-Uni) et Malaga (Espagne) : UICN. xiii + 141 pp.

- Riservato, E. et al. (2009). Statut de conservation et répartition géographique des libellules du bassin méditerranéen. Gland (Suisse) et Malaga (Espagne) : UICN. vii + 33 pp

- Smith, Kevin G. et Darwall, William R.T. (Compilateurs) (2006). The Status and Distribution of Freshwater Fish Endemic to the Mediterranean Basin. UICN, Gland (Suisse) et Cambridge (Royaume-Uni). v + 34 pp.

- Smith, K.G., Barrios, V., Darwall, W.R.T. et Numa, C. (eds) (2014.). Status and distribution of freshwater biodiversity in the eastern Mediterranean. UICN, Cambridge (Royaume-Uni), Malaga (Espagne) et Gland (Suisse) : xiv + 132 pp. 
La délimitation des ZCB d'eau douce en utilisant les limites des sous-bassins versants fluviaux ou lacustres est une approche qui, dans une certaine mesure, diffère de celle utilisée pour délimiter les autres ZCB donc ce choix mérite une explication. Les menaces pesant sur les espèces d'eau douce, surtout dans cette région du monde, sont dues principalement aux altérations hydrologiques (barrages et captage de l'eau), aux espèces exotiques envahissantes et à la pollution de l'eau.

Les effets de ce type de menaces ont tendance à se répercuter rapidement au sein des bassins versants, à tel point que les actions de conservation localisées se limitant à des parties d'un bassin versant ne parviennent généralement pas à faire face à ces menaces. Pour cette raison, l'unité de gestion adaptée pour la plupart des ZCB d'eau douce sera un sous-bassin ou un regroupement de sous-bassins reliés entre eux (par exemple Bruno et al. 2014). Le recours à des ensembles de sous-bassins versants avec une résolution HydroBASINS de niveau 8 (couvrant une superficie moyenne de $600 \mathrm{~km}^{2}$ ) pour délimiter les ZCB permet d'identifier des unités de gestion réalistes. Par exemple, pour soutenir cette approche, considérons une petite source abritant un certain nombre d'escargots endémiques. Dans ce cas précis, la principale menace provient du captage excessif de l'eau à des fins d'irrigation, conduisant à une baisse de la nappe phréatique et à une réduction de l'arrivée d'eau vers la source. La protection de cette source en posant une clôture autour d'elle et en empêchant l'accès à cette source peut sembler être une approche utile mais les niveaux d'eau continueront de baisser, menaçant les escargots. L'important problème de la gestion hydrologique pour l'ensemble du bassin versant n'est toujours pas réglé. En délimitant les ZCB d'eau douce en unités de bassins versants, notre objectif est d'adresser un signal fort affirmant que les écosystèmes d'eau douce doivent être gérés à l'échelle des bassins versants et pas simplement en tant que sites localisés au sein d'une plus grande unité reliée hydrologiquement.

Étape 2. Consultation sur les ZCB proposées (identifiées à l'étape 1) auprès des parties prenantes et délimitation des ZCB validées.

La base de données des ZCB proposées (identifiées à l'étape 1) a été présentée aux parties prenantes lors d'une série d'ateliers, afin d'identifier et de délimiter les bassins versants (ou groupes de bassins versants) remplissant les critères de ZCB dans leur ensemble. Pour la validation de la part des parties prenantes, nous avons d'abord classé les bassins versants par ordre de priorité en fonction du nombre d'espèces de déclenchement remplissant les critères de ZCB, présentes dans chacun d'entre eux. Nous avons aussi classé par ordre de priorité les sites susceptibles d'être admis en tant que sites AZE (" Alliance for Zero Extinction ») (Ricketts et al. 2005). Les sites AZE sont des lieux où les espèces En danger critique ou En danger sont restreintes à d'uniques sites restants. Les parties prenantes ont examiné la liste des bassins versants prioritaires en évaluant

Figure 2. Exemple de carte illustrant la délimitation HydroBASINS de niveau 8.

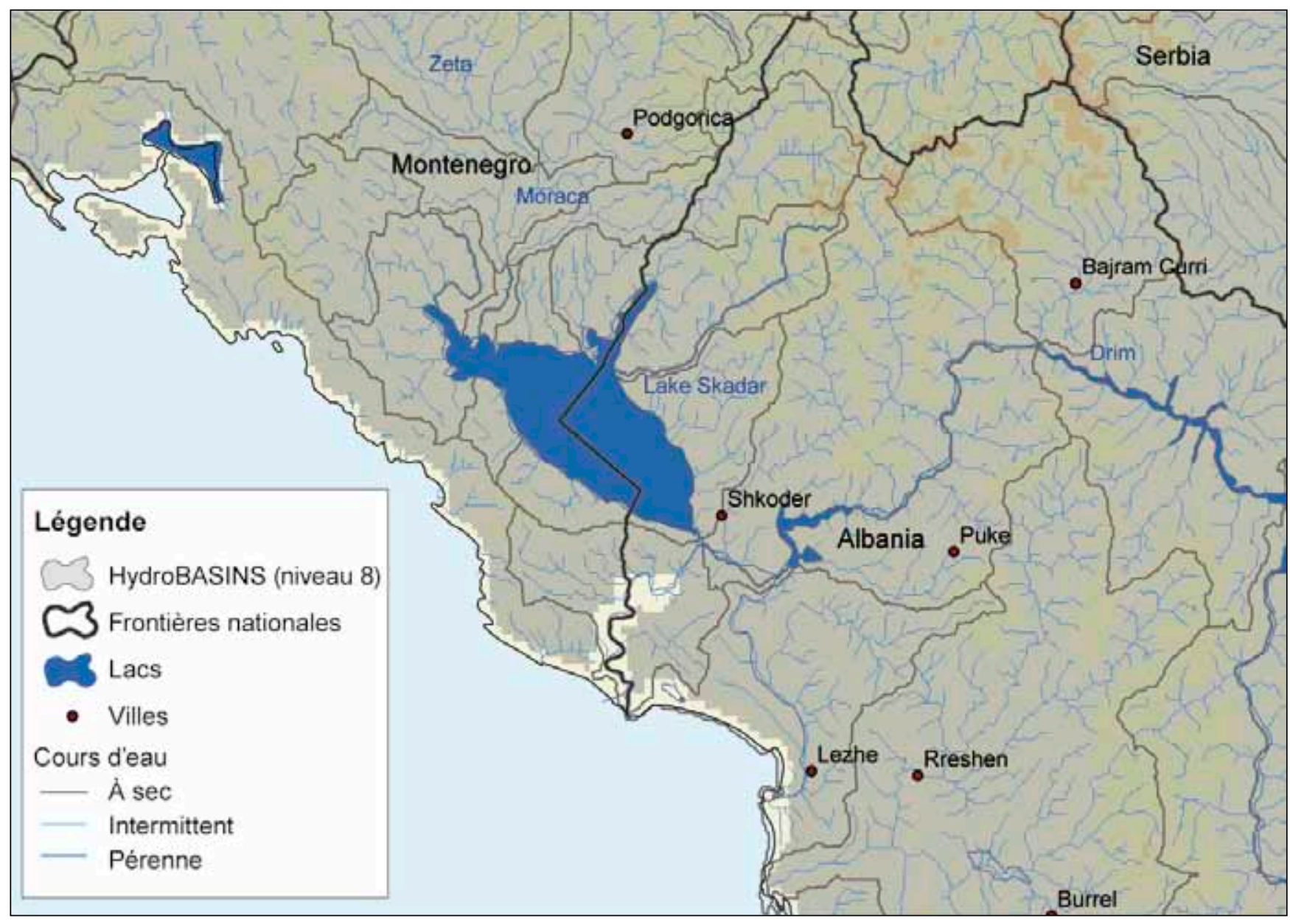


un maximum d'entre eux dans le temps imparti. Du temps supplémentaire a ensuite été accordé aux parties prenantes pour étudier à nouveau la liste complète des ZCB proposées et consulter d'autres parties prenantes pour garantir que l'ensemble des bassins versants les plus importants avait bien été évalué. Tout bassin versant qui n'avait pas encore été évalué mais que les parties prenantes avaient jugé important a aussi été évalué à ce stade, par rapport aux critères de ZCB.

Les parties prenantes ont été identifiées comme étant les personnes/organisations possédant des connaissances scientifiques, impliquées dans les aires protégées ou la gestion de la conservation, et représentant les intérêts du secteur privé dans la région. Lors de chaque atelier, les points suivants ont été discutés et enregistrés à partir des données présentées pour chacune des ZCB proposées, afin de déterminer si celles-ci devaient être confirmées en tant que ZCB validées :

- Présence d'espèces de déclenchement de ZCB - c'està-dire confirmer la présence d'espèces de déclenchement dans le bassin versant, dans la mesure où l'espèce de déclenchement est définie en tant qu'espèce remplissant un ou plusieurs critères de ZCB et déclenchant ainsi l'admission potentielle du ou des bassins versants en tant que ZCB.

- Délimitation du site - déterminer si le bassin versant devrait être délimité en tant que sous-bassin versant unique ou être fusionné avec des bassins versants adjacents pour constituer une unité de gestion de ZCB plus logique.

- Nom du site ZCB - attribuer un nom en fonction des grands cours d'eau, lacs ou zones humides présents dans le bassin versant.

- Description textuelle générale du site - résumer les principales caractéristiques écologiques de la ZCB.

- Zones focales (le cas échéant) - identifier et délimiter les Zones focales au sein des ZCB, dans la mesure où une Zone focale est définie en tant que zone (section de cours d'eau particulier, lac, cours d'eau d'amont, source, zone humide, etc.) située au sein d'une ZCB d'eau douce d'importance particulière concernant une ou plusieurs espèces de déclenchement de ZCB. Par exemple, une Zone focale peut contenir l'ensemble ou la majorité de la population d'une ou de plusieurs espèces de déclenchement, ou bien il s'agit de la seule frayère ou route migratoire connue. Les activités de gestion recommandées peuvent accorder une attention plus particulière aux Zones focales dans le cadre de la gestion et/ ou de la surveillance continue de la ZCB dans son ensemble.

- Chevauchement spatial de la ZCB avec des Aires protégées ou d'autres aires de conservation - confirmer l'étendue du chevauchement spatial entre la ZCB et toute aire protégée existante ou autre aire de conservation, en indiquant l'ampleur de la protection éventuelle de la biodiversité d'eau douce dans ces aires de chevauchement.

- Menaces - identifier les principales menaces pesant sur la biodiversité d'eau douce dans la ZCB.

- Habitat - identifier les principaux habitats d'eau douce présents dans la ZCB.

- Actions de conservation - identifier les actions de conservation déjà en place et proposer de nouvelles actions.

- Parties prenantes de la ZCB - proposer des organisations ou personnes à envisager comme " ambassadeurs locaux » potentiels pour chaque ZCB (c.-à-d. pouvant mener des actions de conservation ou accroître la visibilité de la ZCB) ou ayant un impact ou des intérêts à l'égard de cette ZCB.

L'Annexe I fournit des exemples de fiches techniques relatives aux ZCB.

\section{Analyse des lacunes à l'égard des niveaux actuels de protection}

Pour l'analyse des lacunes relatives à l'inclusion des ZCB d'eau douce au sein des aires protégées existantes et des autres ZCB, nous avons utilisé la série de données provenant de la base de données mondiale des Aires protégées (WDPA, World Database of Protected Areas) (UICN et PNUE-WCMC 2014). II s'agit de la couche de données qui a été utilisée pour calculer les statistiques de couverture des aires protégées pour le rapport Protected Planet report 2014. Cette couche a reçu un traitement préalable qui a supprimé tous les points et polygones de STATUT = « non signalé » et de STATUT = « proposé », et toutes les réserves de biosphère UNESCO MAB car ces éléments incluaient de vastes zones ne correspondant pas à la définition des aires protégées (Juffe-Bignoli et al. 2014). De plus, nous avons intégré la couche spatiale des ZCB pour la Turquie et les couches spatiales des ZCB terrestres fournies par le CEPF. 


\section{Résultats}

\section{Identification des sous-bassins versants fluviaux/ lacustres répondant aux critères d'admission en tant que ZCB proposées}

Dans tout le hotspot méditerranéen, nous avons étudié 3894 sous-bassins versants (résolution HydroBASINS de niveau 8), couvrant une superficie de $2505560 \mathrm{~km}^{2}$. Parmi eux, 3513 sous-bassins versants ont rempli les critères de ZCB et sont désignés ci-après sous le nom " ZCB proposées " (Figure 3). Cette analyse documentaire révèle que la majorité des bassins versants d'eau douce abritent des espèces menacées ou ayant une aire de répartition restreinte, ou bien des assemblages représentatifs d'espèces endémiques, confirmant l'importance et l'urgence de la création et de la mise en œuvre d'actions de conservation pour la biodiversité d'eau douce dans l'ensemble du hotspot. La présence de ces espèces dans des bassins versants individuels a été confirmée par les consultations menées auprès des parties prenantes.

\section{Consultation des parties prenantes et délimitation des ZCB validées}

Trois ateliers ont été organisés entre décembre 2012 et décembre 2013 en Bosnie-Herzégovine, au Maroc et en Jordanie (voir précisions dans le Tableau 2 ci-dessous), pour impliquer les parties prenantes concernées et les experts en biodiversité (Annexe II). Ces ateliers ont été axés sur la validation des ZCB proposées en procédant à : i) la confirmation de la présence d'espèces de déclenchement ; ii) l'identification et la documentation des menaces pesant sur les sous-bassins versants ; iii) l'identification et la documentation des besoins en termes de conservation ; iv) la délimitation de l'étendue des ZCB de manière pertinente, et ; v) l'identification des « Ambassadeurs locaux » pour chaque ZCB.

Tableau 2. Ateliers dédiés à la validation des ZCB d'eau douce pour le sud et l'est de la Méditerranée.

\begin{tabular}{|l|c|c|c|}
\hline Sous-région méditerranéenne & \multicolumn{2}{c}{ Dates } & Durée \\
\hline Balkans & Jahorina (Bosnie-Herzégovine) & $11-13$ décembre 2012 & 3 jours \\
\hline Afrique du Nord & Marrakech (Maroc) & $03-06$ septembre 2013 & 3 jours \\
\hline Turquie et Levant & Azraq (Jordanie) & $02-06$ décembre 2013 & 5 jours \\
\hline
\end{tabular}

Figure 3. Ensemble des ZCB proposées avec indication du nombre d'espèces de déclenchement de ZCB dans chaque sous-bassin versant de la région du hotspot méditerranéen.

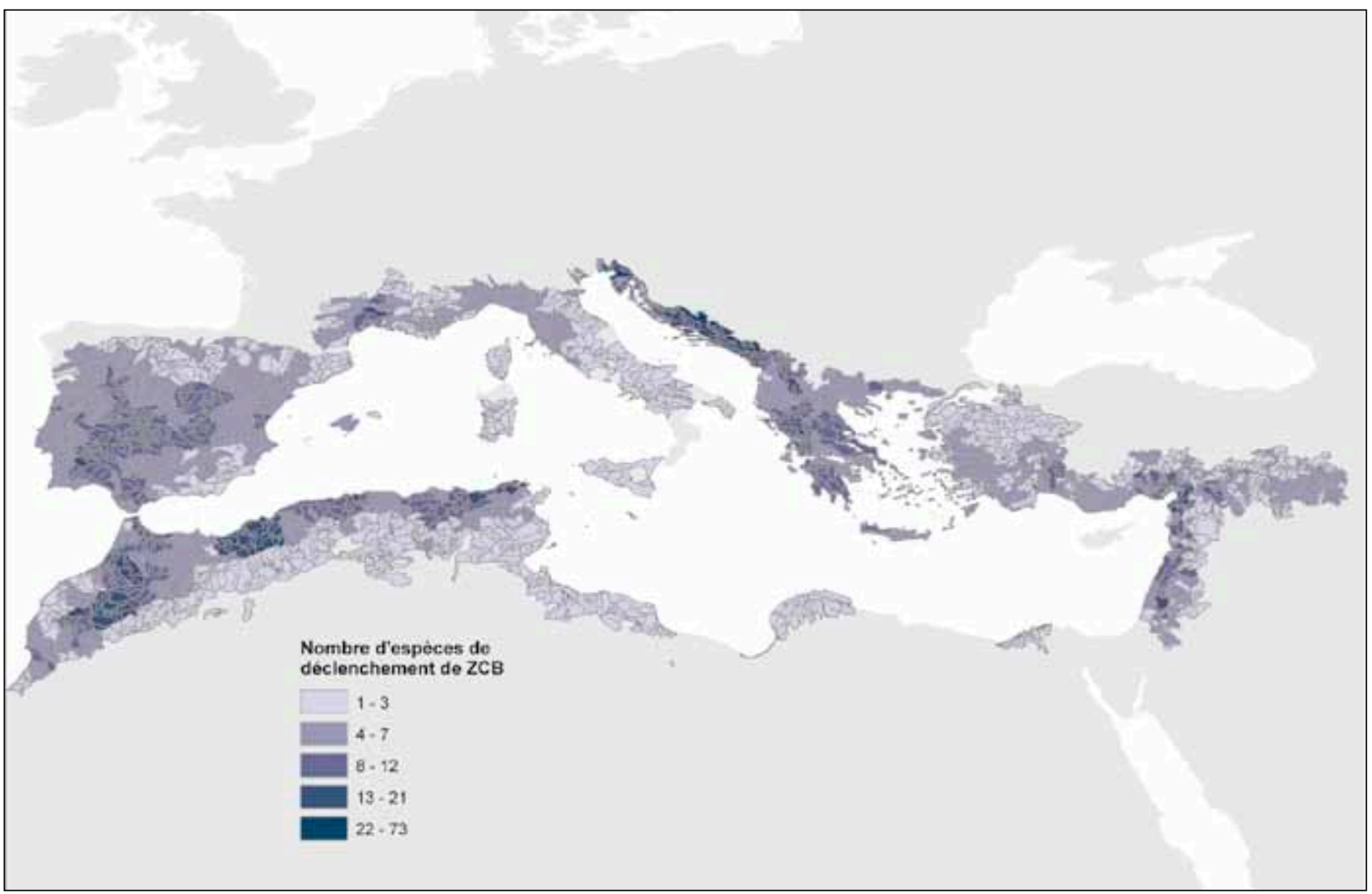




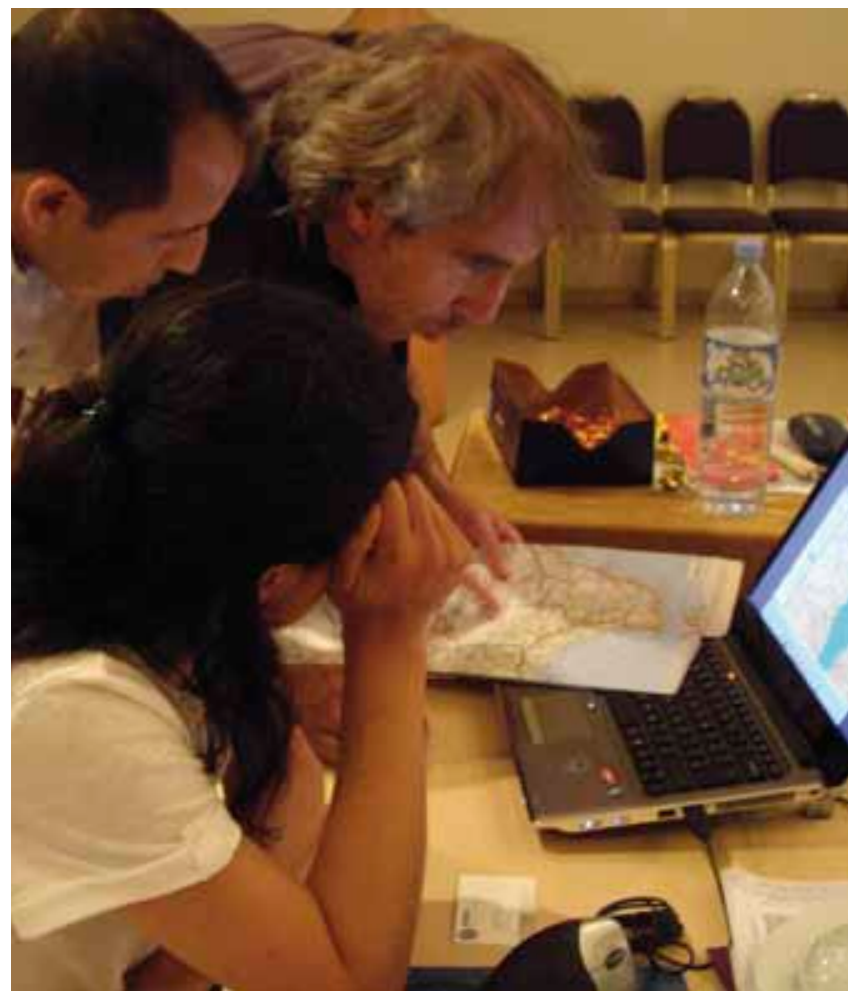

Participants discutant des sites de ZCB d'eau douce potentiels lors de l'atelier avec les parties prenantes sur la délimitation et la validation des ZCB pour la sous-région de l'Afrique du Nord.

(c) Violeta Barrios

\section{ZCB d'eau douce validées}

Lors de ces trois ateliers, 167 ZCB d'eau douce, couvrant une superficie totale de $302557 \mathbf{k m}^{2}$, ont été validées et délimitées pour le sud et l'est de la Méditerranée (voir Tableau 3 et Figure 4). Les fiches techniques concernant chaque ZCB peuvent être consultées dans la base de données pour la biodiversité mondiale (WBDB, World Biodiversity DataBase) gérée par BirdLife International (http://www.birdlife.org/datazone/ freshwater). Les 1368 ZCB proposées pour la péninsule Ibérique, la France et l'Italie doivent encore être classées par ordre de priorité, affinées et validées en tant que ZCB. De plus, une évaluation supplémentaire a été menée pour couvrir la zone située en dehors des limites du hotspot de la biodiversité du bassin méditerranéen car un certain nombre d'espèces ont une aire de répartition s'étendant à des bassins versants reliés hydrologiquement à cette région élargie. Un état des ZCB d'eau douce de la zone orientale élargie de la Méditerranée, intégrant l'ensemble des bassins reliés au-delà des limites du hotspot, comme le Tigre et l'Euphrate, est présenté à l'Annexe III.

Le réseau de 167 ZCB d'eau douce validées en découlant intègre les aires de répartition de 491 espèces de déclenchement de ZCB (dont 377 sont menacées d'extinction, 411 ont une aire de répartition restreinte et 107 sont restreintes à un biome) (Tableau 3, Figure 4 et Annexe IV).

Figure 4. Ensemble des ZCB validées avec indication du nombre d'espèces de déclenchement de ZCB dans chaque site de la région du hotspot méditerranéen.

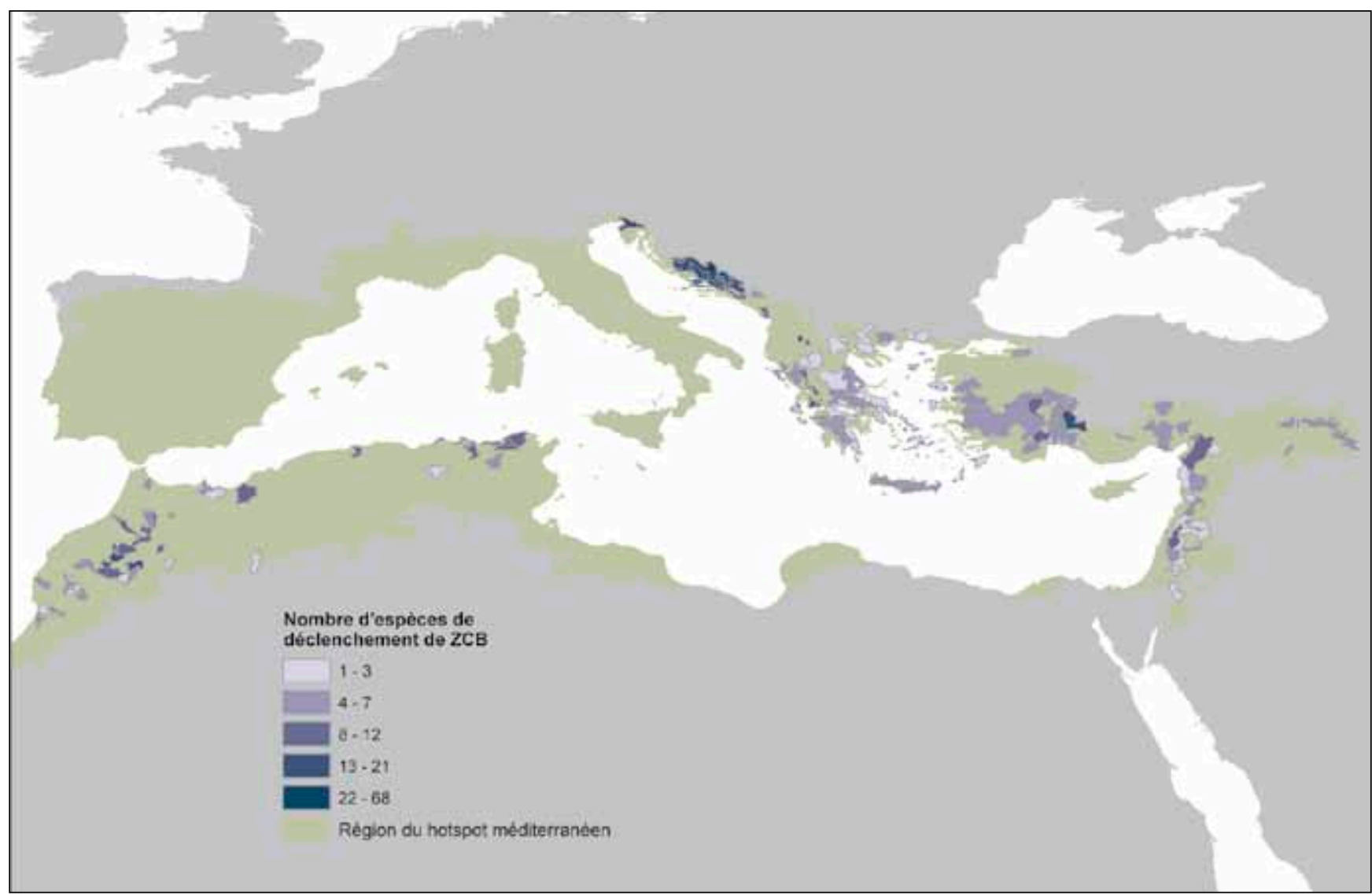


Tableau 3. Nombre de ZCB d'eau douce validées, de sites «Alliance for Zero Extinction » (AZE) et d'espèces menacées, ayant une aire de répartition restreinte, et restreintes à un biome dans les sous-régions méditerranéennes. * Le total comptabilise une seule fois les espèces apparaissant dans plus d'une région, et ne correspond donc pas à la somme des lignes.

\begin{tabular}{|l|c|c|c|c|}
\hline & Balkans & Turquie et Levant & Afrique du Nord & Total \\
\hline Nombre d'espèces menacées & 206 & 105 & 70 & $\mathbf{3 7}^{*}$ \\
\hline $\begin{array}{l}\text { Nombre d'espèces ayant une aire de répartition } \\
\text { restreinte }\end{array}$ & 265 & 97 & 50 & $\mathbf{4 1 1}^{*}$ \\
\hline Nombre d'espèces restreintes à un biome & 67 & 9 & 31 & $\mathbf{1 0 7}^{*}$ \\
\hline Nombre d'espèces de déclenchement de ZCB & 281 & 123 & 92 & 491 \\
\hline Nombre de ZCB d'eau douce validées & 77 & 47 & 43 & $\mathbf{1 6 7}$ \\
\hline Nombre de sites AZE & 20 & 18 & 2 & $\mathbf{4 0}$ \\
\hline
\end{tabular}

La ZCB abritant le plus grand nombre d'espèces de déclenchement est le bassin du lac Ohrid en Albanie et dans l'ancienne République yougoslave de Macédoine (ARYM) (68 espèces de déclenchement), suivie du lac Busko (27 espèces de déclenchement) et des poljés occidentaux de BosnieHerzégovine (27 espèces de déclenchement). Dans l'ensemble du hotspot, 40 sites AZE d'eau douce ont été validés (Figure 5).

\section{Principales menaces}

Les principales menaces pesant sur la biodiversité ont été identifiées pour chaque ZCB d'eau douce validée. En général, elles incluent la gravité accrue des sécheresses, les altérations hydrologiques suite à la construction de barrages, le captage excessif des eaux souterraines et de surface, la pollution de l'eau et les espèces envahissantes. Les sections suivantes présentent un aperçu de chaque sous-région, en s'intéressant spécifiquement dans chaque cas à un certain nombre de ZCB.

\section{Niveaux actuels de protection des ZCB d'eau douce au sein du hotspot méditerranéen}

Le chevauchement spatial entre, d'une part, les Aires protégées $(A P)$ et d'autres ZCB, et, d'autre part, les ZCB d'eau douce

Figure 5. ZCB validées répondant également aux critères d'admission en tant que sites AZE dans la région du hotspot méditerranéen.

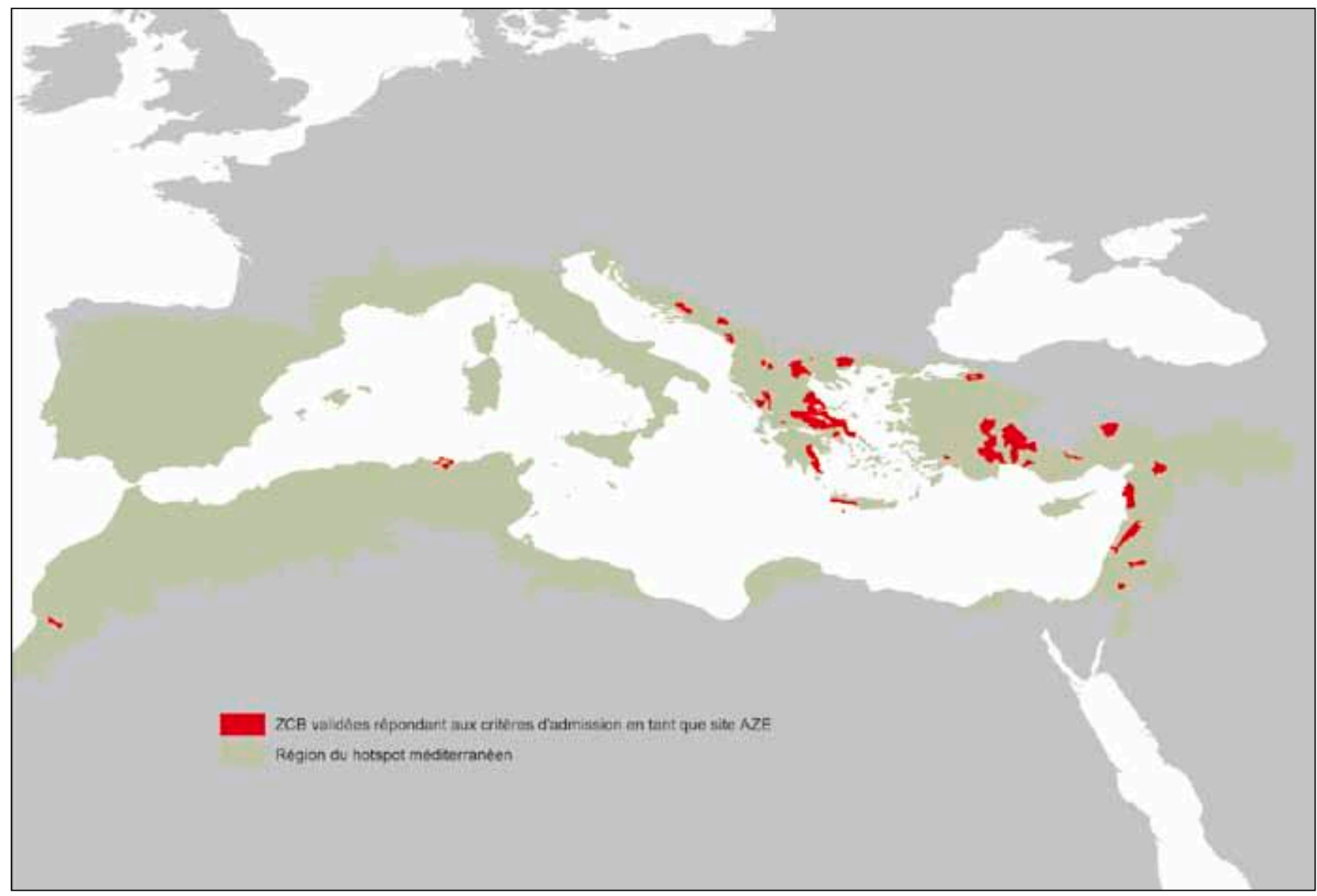




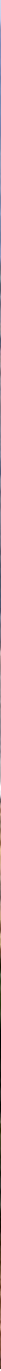

La construction et la gestion des barrages, comme le barrage d'Ouirgane sur l'oued N'Fiss au Maroc, tiennent rarement compte de leur impact sur la biodiversité d'eau douce. Les politiques en matière de gestion des ressources en eau dans le bassin méditerranéen, par exemple pour l'exploitation hydroélectrique, doivent intégrer de nouvelles mesures visant à préserver le fonctionnement écologique des écosystèmes d'eau douce. @ William Darwall

La ZCB du poljé de Popovo et de la Trebišnjica [nom anglais : Popovo polje and Trebišnjica] est l'un des plus vastes poljés (plaines karstiques) de Bosnie-Herzégovine, et est connue pour la rivière Trebišnjica qui traverse ce poljé, alimentant les eaux souterraines, et pour le réseau de grottes de Vjetrenica qui abritent une faune cavernicole très riche. Les poljés comme celui-ci sont gravement affectés par les altérations hydrologiques continues qu'ils subissent notamment aux fins du développement de l'hydroélectricité (c Biciklima za odrzivu energiju 2011. Online image/Flickr (CC BY-NC-ND 2.0)

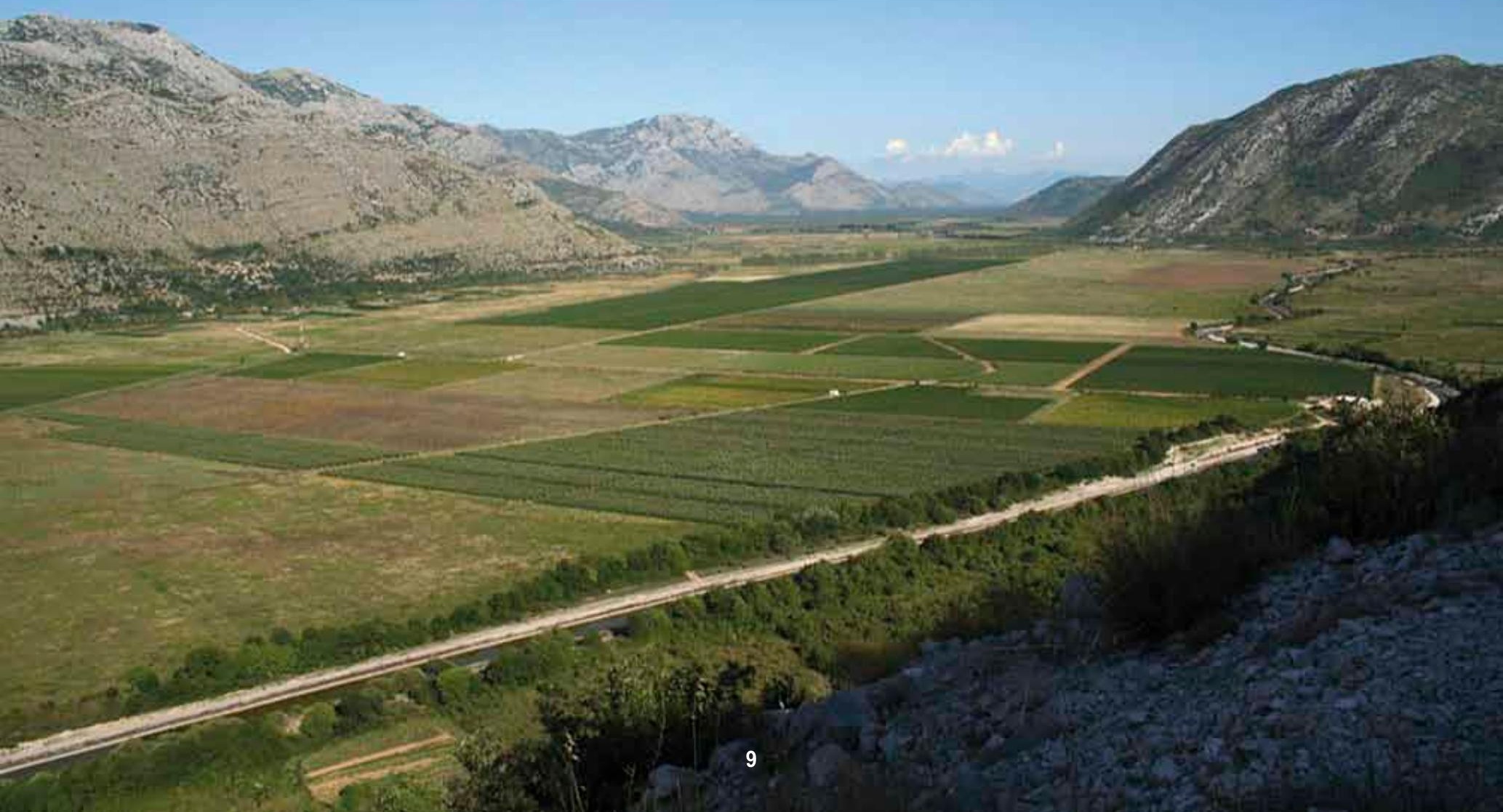


validées, couvre une superficie totale de $76462,06 \mathrm{~km}^{2}$ (25\% de la superficie totale des ZCB d'eau douce validées) (Figure 6). Ceci signifie que $75 \%$ de la superficie des ZCB d'eau douce validées se situent en dehors des limites géographiques des $A P$ ou ZCB existantes.

Dans la région, les $\mathrm{ZCB}^{1}$ d'eau douce validées suivantes se trouvent en dehors des limites géographiques des autres ZCB ou AP existantes :

1. Amman (Jordanie)

2. Assif El Mal est (Maroc) [nom anglais : Assif El Mal east]

3. Beni Belaid (Algérie)

4. Bassin versant autour de Niksic (Monténégro) [nom anglais : Catchment surrounding Niksic]

5. Lac Bilecko (Bosnie-Herzégovine) [nom anglais : Lake Bilecko]

6. Lac Kastrakiou (Grèce) [nom anglais : Lake Kastrakiou]

7. Rivière Listica et Mostarsko blato (Bosnie-Herzégovine) [nom anglais : Listica River and Mostarsko blato]

8. Bassin de l'oued M'Goun (Maroc) [nom anglais : M'Goun river basin]

9. Poljé de Nevesinjsko, poljé de Gatacko, poljé de Cernicko, poljé de Fatnicko et poljé de Dabarsko (BosnieHerzégovine et Monténégro) [nom anglais : Nevesinjsko polje, Gatacko polje, Cernicko polje, Fatnicko polje and Dabarsko polje]

10. Oued Zhour (Algérie)

11. Partie du bassin versant supérieur du fleuve Neretva (Bosnie-Herzégovine) [nom anglais : Part of the Neretva upper catchment]

12. Partie du bassin versant supérieur du fleuve Neretva - bassin moyen oriental (Bosnie-Herzégovine) [nom anglais : Part of the Neretva upper catchment - eastern mid catchment]

13. Poljé de Popovo et Trebišnjica (Bosnie-Herzégovine, Monténégro et Croatie) [nom anglais : Popovo polje and Trebišnjica]
14. Affluents du cours supérieur et moyen du Neretva y compris Hutovo Blato (Bosnie-Herzégovine) [nom anglais : Tributaries of lower and middle Neretva including Hutovo Blato]

15. Cours supérieur du Medjarda (Algérie et Tunisie) [nom anglais : Upper Medjarda River].

Aucune analyse des lacunes n'a été effectuée pour la sousrégion du nord-ouest de la Méditerranée car ces sites n'ont pas encore été validés en tant que ZCB.

\section{Ambassadeurs locaux}

Les ZCB d'eau douce ont à présent été identifiées, cartographiées et validées dans une grande partie du hotspot méditerranéen, et les informations concernant chaque site peuvent être consultées par le public grâce à la base de données pour la biodiversité mondiale (WBDB, World Biodiversity DataBase) et à l'outil intégré d'évaluation de la biodiversité (IBAT, Integrated Biodiversity Assessment Tool). Nous avons maintenant besoin que des personnes/organisations prennent les commandes et concrétisent sur le terrain les actions recommandées pour la préservation des ZCB. Les "Ambassadeurs locaux » ont été identifiés lors des ateliers des parties prenantes comme étant les personnes/organisations les mieux placées pour sensibiliser à l'existence des ZCB et aux difficultés rencontrées en raison des menaces pesant sur la biodiversité, et pour aider à mettre en œuvre les actions requises en vue de préserver ces sites dont l'importance est de portée mondiale.

Au total, 188 ambassadeurs locaux potentiels et parties prenantes ont été recommandés pour les différentes ZCB (voir Annexe V).

1 N.D.T. : Les noms des sites ZCB figurant dans les tableaux sont en anglais. Toutefois, les noms des sites ZCB cités dans le corps de ce document ont été traduits en français en précisant leur nom anglais entre crochets (« [nom anglais : $X X X]$ »), afin de permettre au lecteur de faire le lien avec les noms des sites en anglais figurant dans les tableaux correspondants et dans la base de données en ligne (WBDB, World Biodiversity DataBase). 







\section{Les ZCB d'eau douce dans la sous-région des Balkans}

Cette région (Figure 7) intègre les deux corridors prioritaires définis par le CEPF, l'Adriatique orientale et le sud-ouest des Balkans. Le corridor de l'Adriatique orientale traverse la Bosnie-Herzégovine, la Croatie et le Monténégro, et cette zone est caractérisée par ses nombreuses grottes, sources et rivières karstiques, et par son poljé associé (dépressions typiques des régions karstiques, comprenant généralement des marais ou de petits lacs) qui revêt une réelle importance pour un certain nombre d'espèces d'eau douce dont beaucoup sont endémiques. Les principales menaces pesant sur les écosystèmes d'eau douce proviennent de l'exploitation hydroélectrique, le détournement de l'eau pour l'agriculture, l'aménagement des terres dans les bassins versants supérieurs, et le tourisme côtier. Les espèces envahissantes représentent aussi une menace potentielle dans de nombreuses zones. Le corridor du sud-ouest des Balkans inclut certaines parties de l'Albanie, de l'ARYM (ancienne République yougoslave de Macédoine), de la Grèce, du Monténégro et de la Serbie. Ce corridor a été identifié principalement pour la biodiversité d'eau douce unique de cette région. On y trouve trois principaux systèmes lacustres : les systèmes des lacs Prespa et Ohrid partagés par la Grèce, l'ARYM et l'Albanie, le système du lac
Skadar partagé par l'Albanie et le Monténégro, et la zone du lac Dojran partagée par l'ARYM et la Grèce.

\section{Aperçu des ZCB}

Pour la sous-région des Balkans, nous avons étudié 528 sousbassins versants (résolution HydroBASINS de niveau 8), couvrant une superficie de $216492 \mathrm{~km}^{2}$. Au sein de ces sousbassins versants, 253 ZCB proposées ont été identifiées comme remplissant les critères de ZCB en raison des groupes taxonomiques d'eau douce évalués à cet endroit (Figure 8 , Tableau 4). Concernant les espèces de déclenchement de ZCB, 306 espèces de ce type ont été identifiées. Une consultation ultérieure auprès des parties prenantes concernant ces ZCB proposées a conduit à la désignation et à la délimitation de 77 ZCB validées (Figure 9, Tableau 5 et Tableau 6). Cet ensemble de ZCB abrite le plus grand nombre d'espèces menacées, ayant une aire de répartition restreinte, et restreintes à un biome dans les sous-régions évaluées. Ainsi, 281 espèces remplissent au moins l'un des critères de ZCB (Annexe IV, Tableau A).

\section{Niveaux actuels de protection}

La zone des ZCB d'eau douce validées se trouvant dans les limites géographiques des aires protégées (AP) existantes et des

Figure 7. La sous-région des Balkans.

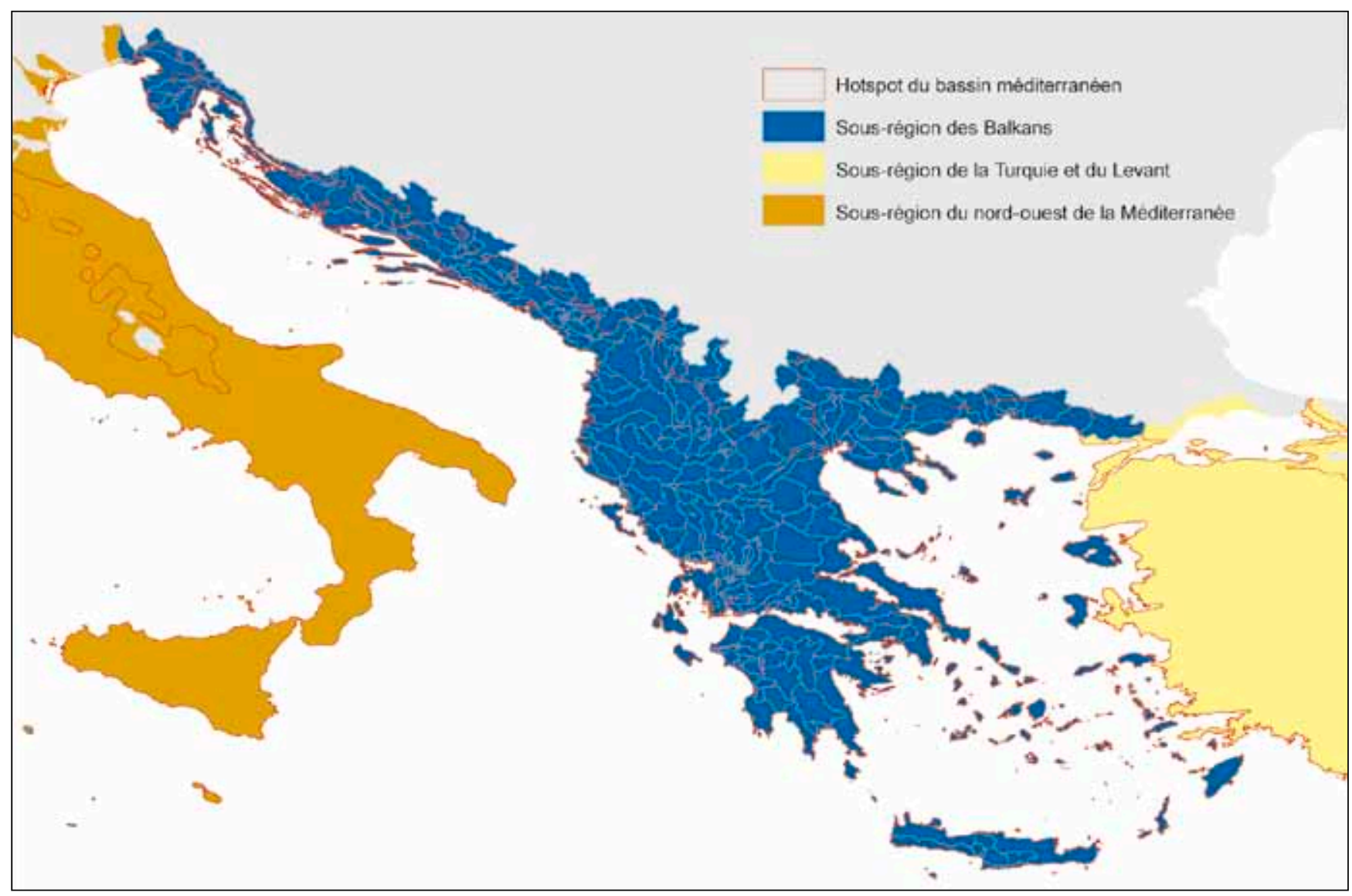


autres ZCB représente $36948 \mathrm{~km}^{2}$ (36\% de la superficie totale des ZCB d'eau douce validées dans la sous-région des Balkans).

Tableau 4. Synthèse des ZCB proposées. Nombre de sous-bassins versants proposés en tant que $\mathrm{ZCB}$ en raison de la présence d'espèces menacées (C1), d'espèces ayant une aire de répartition restreinte (C2), et de communautés restreintes à un biome (C3) pour chaque groupe taxonomique. À noter : les chiffres de la colonne "Tous les critères " représentent le nombre total de bassins versants distincts qualifiés, et ne correspondent donc pas à la somme des lignes.

\begin{tabular}{|lcccc|}
\hline & \multicolumn{4}{c}{ Nombre de sous-bassins versants qualifiés } \\
\hline & Tous les critères & C1 & C2 & C3 \\
\hline Poissons & 211 & 156 & 201 & 3 \\
\hline Mollusques & 197 & 139 & 191 & 1 \\
\hline Odonates & 26 & 26 & 23 & 0 \\
\hline Plantes & 81 & 78 & 4 & 0 \\
\hline Total & $\mathbf{2 5 3}$ & $\mathbf{2 3 1}$ & $\mathbf{2 4 5}$ & $\mathbf{3}$ \\
\hline
\end{tabular}

Tableau 5. Synthèse des ZCB validées. Nombre de sous-bassins versants validés en raison de la présence d'espèces menacées (C1), d'espèces ayant une aire de répartition restreinte (C2), et de communautés restreintes à un biome (C3) pour chaque groupe taxonomique. À noter : les chiffres de la colonne "Tous les critères " représentent le nombre total de bassins versants distincts qualifiés, et ne correspondent donc pas à la somme des lignes.

\begin{tabular}{|lcccc|}
\hline & \multicolumn{4}{c}{ Nombre de sous-bassins versants qualifiés } \\
\hline Tous les critères & C1 & C2 & C3 \\
\hline Poissons & 63 & 54 & 59 & 2 \\
\hline Mollusques & 62 & 44 & 60 & 1 \\
\hline Odonates & 13 & 13 & 11 & 0 \\
\hline Plantes & 14 & 11 & 4 & 0 \\
\hline Total & $\mathbf{7 7}$ & $\mathbf{7 0}$ & $\mathbf{7 4}$ & $\mathbf{2}$ \\
\hline
\end{tabular}

Ceci signifie que $64 \%$ de la superficie de ces ZCB d'eau douce se situent à l'extérieur des aires protégées officielles ou d'autres sites reconnus pour leur importance biologique en tant que ZCB (Figure 10).

En outre, les ZCB d'eau douce validées suivantes se trouvent complètement en dehors des limites géographiques des ZCB ou AP existantes :

1. Bassin versant autour de Niksic (Monténégro) [nom anglais : Catchment surrounding Niksic]

2. Lac Bilecko (Bosnie-Herzégovine) [nom anglais : Lake Bilecko]

3. Lac Kastrakiou (Grèce) [nom anglais : Lake Kastrakiou]

4. Rivière Listica et Mostarsko blato (Bosnie-Herzégovine) [nom anglais : Listica River and Mostarsko blato]

5. Poljé de Nevesinjsko, poljé de Gatacko, poljé de Cernicko, poljé de Fatnicko et poljé de Dabarsko (Bosnie-Herzégovine et Monténégro) [nom anglais : Nevesinjsko polje, Gatacko polje, Cernicko polje, Fatnicko polje and Dabarsko polje]

6. Partie du bassin versant supérieur du fleuve Neretva (Bosnie-Herzégovine) [nom anglais : Part of the Neretva upper catchment]

7. Partie du bassin versant supérieur du fleuve Neretva bassin moyen oriental (Bosnie-Herzégovine) [nom anglais : Part of the Neretva upper catchment - eastern mid catchment]

8. Poljé de Popovo et Trebišnjica (Bosnie-Herzégovine, Monténégro et Croatie) [nom anglais : Popovo polje and Trebišnjica]

9. Affluents du cours supérieur et moyen du Neretva y compris Hutovo Blato (Bosnie-Herzégovine) [nom anglais : Tributaries of lower and middle Neretva including Hutovo Blato].

Figure 8. Ensemble des ZCB proposées avec indication du nombre d'espèces de déclenchement de ZCB dans la sous-région des Balkans.

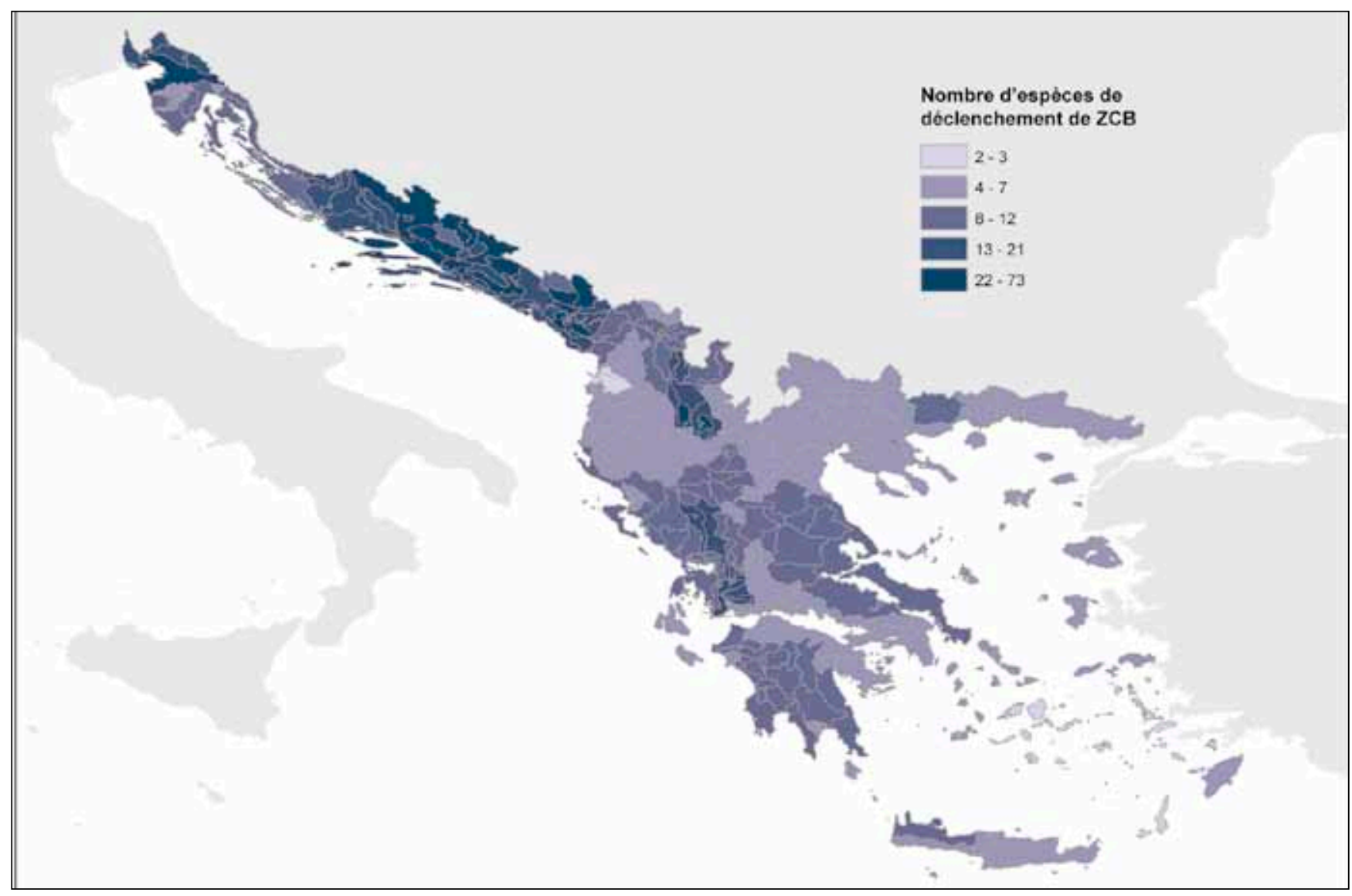




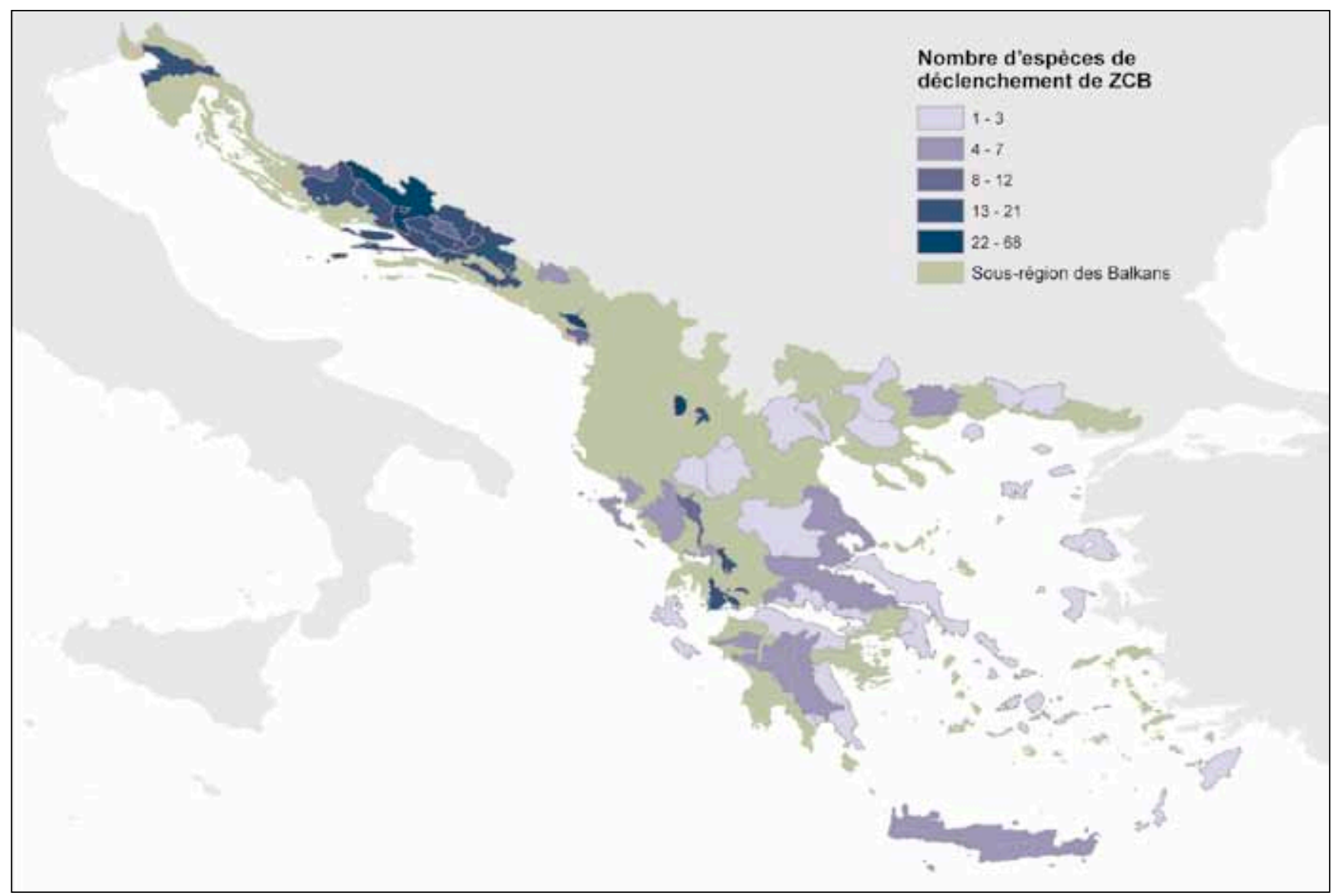

Figure 9. Ensemble des ZCB validées avec indication du nombre d'espèces de déclenchement de ZCB dans la sous-région des Balkans.

Tableau 6. ZCB d'eau douce validées pour les sous-bassins versants de la sous-région des Balkans, indiquant le nombre d'espèces de déclenchement de ZCB, les sites AZE (*) et, si elles ont été identifiées, les Zones focales pour chaque ZCB. Les ZCB transfrontalières figurent à la fin du tableau.

\begin{tabular}{|c|c|c|c|}
\hline Pays & Nom de la ZCB ${ }^{1}$ & $\begin{array}{l}\text { Nombre } \\
\text { d'espèces de } \\
\text { déclenchement }\end{array}$ & Zones focales ${ }^{2}$ \\
\hline Albanie & Butrint & 4 & \\
\hline Bosnie-Herzégovine & Lake Bilecko & 21 & \\
\hline Bosnie-Herzégovine & Lake Busko & 27 & \\
\hline Bosnie-Herzégovine & Listica River and Mostarsko blato & 8 & \\
\hline Bosnie-Herzégovine & $\begin{array}{l}\text { Nevesinjsko polje, Gatacko polje, Cernicko polje, Fatnicko } \\
\text { polje and Dabarsko polje }\end{array}$ & 19 & Poljé de Fatnicko et poljé de Dabarsko \\
\hline Bosnie-Herzégovine & Part of the Neretva upper catchment & 21 & \\
\hline Bosnie-Herzégovine & $\begin{array}{l}\text { Part of the Neretva upper catchment - eastern mid } \\
\text { catchment }\end{array}$ & 18 & \\
\hline Bosnie-Herzégovine & Popovo polje and Trebisnjica & 21 & $\begin{array}{l}\text { Grotte de Vjetrenica, grotte de Doljasnica, } \\
\text { grotte de Ponikva, grotte de Lisac et grotte } \\
\text { de Crnulja }\end{array}$ \\
\hline Bosnie-Herzégovine & Tributaries of lower and middle Neretva & 18 & $\begin{array}{l}\text { Fleuve Neretva au sud de Mostar, rivières } \\
\text { Bregava et Buna dont Hutovo Blato }\end{array}$ \\
\hline Bosnie-Herzégovine & West Karst poljes & 27 & \\
\hline Croatie & Cetina River & 15 & \\
\hline Croatie & Matica River and Bacina lakes & 19 & \\
\hline Croatie & Zrmanja River & 10 & \\
\hline Grèce & Acheron* & 4 & \\
\hline Grèce & Aggitis* $^{*}$ & 5 & \\
\hline Grèce & Aliakmon Naoussa* & 1 & \\
\hline Grèce & Andros Tinos & 1 & \\
\hline
\end{tabular}


Tableau 6. ZCB d'eau douce validées pour les sous-bassins versants de la sous-région des Balkans (suite).

\begin{tabular}{|c|c|c|}
\hline Pays & Nom de la ZCB' & $\begin{array}{l}\text { Nombre } \\
\text { d'espèces de } \\
\text { déclenchement Zones focales }{ }^{2}\end{array}$ \\
\hline Grèce & Arachthos & 5 \\
\hline Grèce & Arkadia Plateau & 5 \\
\hline Grèce & Chios & 1 \\
\hline Grèce & Corfu Island (Kerkyra) & 6 \\
\hline Grèce & Crete central south & 5 \\
\hline Grèce & Crete eastern & 5 \\
\hline Grèce & Crete north-west & 6 \\
\hline Grèce & Crete south-west ${ }^{\star}$ & 6 \\
\hline Grèce & Eastern Attica & 1 \\
\hline Grèce & Euboea Manikiatis* & 3 \\
\hline Grèce & Evrotas & 4 \\
\hline Grèce & Evrotas-Arniotikos & 1 \\
\hline Grèce & Evrotas-Gytheio & 3 \\
\hline Grèce & Ismaris-Vosvozis-Filiouris & 3 \\
\hline Grèce & Kalamas & 5 \\
\hline Grèce & Karla* $^{*}$ & 4 \\
\hline Grèce & Karpathos & 1 \\
\hline Grèce & Kastoria & 2 \\
\hline Grèce & Kastraki & 15 \\
\hline Grèce & Kephalonia and Ithaki & 1 \\
\hline Grèce & Kerkini & 1 \\
\hline Grèce & Krka drainage & 14 \\
\hline Grèce & Ladon & 4 \\
\hline Grèce & Lake Kastrakiou & 16 \\
\hline Grèce & $\begin{array}{l}\text { Lakes Limnothalassa Rodias, Limnothalassa Tsoukaliou, } \\
\text { Limnothalassa Lagarou }\end{array}$ & 7 \\
\hline Grèce & Lakes Trichonis and Lisimachia* & 18 \\
\hline Grèce & Lesvos & 2 \\
\hline Grèce & Lower Acheloos & 16 \\
\hline Grèce & Lower Alfeios & 4 \\
\hline Grèce & Lower Axios & 1 \\
\hline Grèce & Magnisia* & 5 \\
\hline Grèce & Mornos & 5 \\
\hline Grèce & Naxos & 1 \\
\hline Grèce & Northern Korinthiakos & 1 \\
\hline Grèce & Pamvotis Lake* & 10 \\
\hline Grèce & Peloponnese Maleas* & 1 \\
\hline Grèce & Pineios Peloponnisou & 4 \\
\hline Grèce & Pinios Thessalias & 3 \\
\hline Grèce & Rhodes Island & 1 \\
\hline Grèce & Spercheios* $^{*}$ & 4 \\
\hline Grèce & Tempi & 5 \\
\hline Grèce & Thassos & 2 \\
\hline Grèce & Tragos & 4 \\
\hline Grèce & Upper Alfeios & 5 \\
\hline Grèce & Upper Aliakmon & 2 \\
\hline Grèce & Upper Aoos & 3 \\
\hline Grèce & Upper Kifissos* & 1 \\
\hline Grèce & Vistonis & 2 \\
\hline
\end{tabular}


Tableau 6. ZCB d'eau douce validées pour les sous-bassins versants de la sous-région des Balkans (suite).

\begin{tabular}{|c|c|c|c|}
\hline Pays & Nom de la ZCB ${ }^{1}$ & $\begin{array}{c}\text { Nombre } \\
\text { d'espèces de } \\
\text { déclenchement }\end{array}$ & Zones focales ${ }^{2}$ \\
\hline Grèce & Volvi-Koronia & 3 & \\
\hline Grèce & Yliki-Paralimni-Kifissos* & 5 & \\
\hline Grèce & Zakynthos & 3 & \\
\hline Monténégro & Catchment surrounding Niksic* & 5 & \\
\hline Albanie, ARYM & Lake Ohrid* & 68 & \\
\hline Albanie, Grèce, ARYM & Transboundary Prespa Park* & 21 & \\
\hline Albanie, Monténégro & Lake Skadar* & 24 & $\begin{array}{l}\text { Le lac en lui-même, certaines sources et les } \\
\text { affluents du lac de moyenne ou grande taille }\end{array}$ \\
\hline Albanie, Monténégro & Lower Bojana river basin* & 12 & \\
\hline $\begin{array}{l}\text { Croatie, Bosnie- } \\
\text { Herzégovine }\end{array}$ & $\begin{array}{l}\text { Neretva Delta and associated springs/lakes including } \\
\text { Hutovo Blato }\end{array}$ & 19 & \\
\hline $\begin{array}{l}\text { Croatie, Bosnie- } \\
\text { Herzégovine }\end{array}$ & Trebizat drainage including Imotsko polje* & 20 & \\
\hline Grèce, ARYM & Doirani* & 2 & \\
\hline Slovénie, Croatie & $\begin{array}{l}\text { Dragonija drainage in Slovenia and Croatia, Reka River in } \\
\text { Slovenia and Timavo spring, north of Trieste in Italy }\end{array}$ & 19 & $\begin{array}{l}\text { Rivière Reka et source du fleuve Timave } \\
\text { (Timavo) }\end{array}$ \\
\hline
\end{tabular}

1 N.D.T. : Les noms des sites ZCB figurant dans les tableaux sont en anglais. Toutefois, les noms des sites ZCB cités dans le corps de ce document ont été traduits en français en précisant leur nom anglais entre crochets (" [nom anglais : XXX] »), afin de permettre au lecteur de faire le lien avec les noms des sites en anglais figurant dans les tableaux correspondants et dans la base de données en ligne (WBDB, World Biodiversity DataBase).

2 Nous ne disposons pas encore de la liste complète des Zones focales pour cette première sous-région car les informations n'ont pas été recueillies systématiquement à l'époque. D'autres Zones focales pourront être définies en consultant les fiches techniques et en nous contactant à l'adresse : freshwater.biodiversity@iucn.org

Une cascade au sein de la ZCB du bassin de la rivière Krka [nom anglais : Krka drainage], qui abrite de nombreux types d'habitats d'eau douce tels que des sources, des lacs, des cascades et des rivières karstiques ainsi que des zones de tufs et des eaux souterraines. Cette ZCB inclut 14 espèces parmi les espèces d'eau douce menacées ou ayant une aire de répartition restreinte. $\odot$ Geert De Knijf

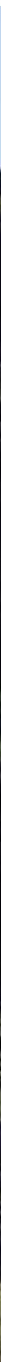




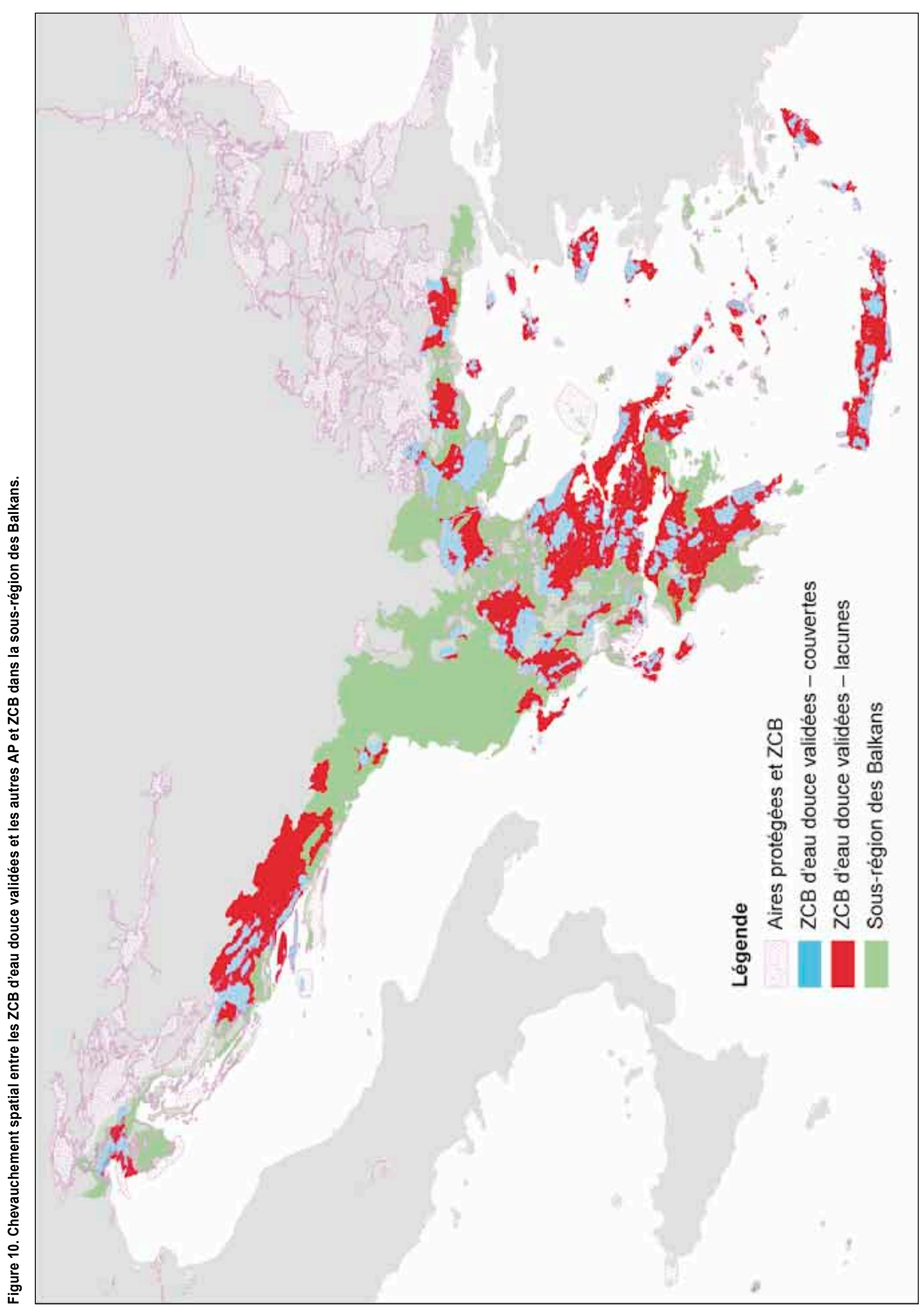




\section{Discussion}

La sous-région des Balkans abrite un certain nombre de systèmes fluviaux et lacustres importants, validés en tant que ZCB d'eau douce, et un grand nombre d'entre elles répondent aussi aux critères d'admission en tant que sites AZE (Figure 5 et Tableau 6). Les difficultés rencontrées par ces sites critiques sont soulignées plus bas, dans le cadre de la discussion sur quelques-uns de ces sites.

Dans le corridor de l'Adriatique orientale, les difficultés rencontrées au niveau de la ZCB du bassin de la rivière Trebizat incluant le poljé d'Imotsko [nom anglais : Trebizat drainage including Imotsko polje], également identifiée en tant que site AZE d'eau douce, sont emblématiques de celles rencontrées par de nombreuses ZCB d'eau douce de la sousrégion. Ce site comprend des sources, des lacs et des rivières karstiques, de nombreuses cascades, des zones de tufs riches en plantes de milieux humides, des aquifères souterrains, ainsi que des zones inondées de manière saisonnière (poljés). Le site abrite 20 espèces de déclenchement composées de poissons et de mollusques. Le Cobitis illyrica (En danger critique), une espèce de loche de rivière, est a priori propre au poljé d'Imotsko (Freyhof and Stelbrink 2007). En raison de cette espèce spécifique, le site répond aux critères d'admission en tant que site AZE. Cette ZCB est principalement menacée par les perturbations hydrologiques provoquées par deux grands barrages hydroélectriques (Pec Mlini en Bosnie-Herzégovine, et Ricice en Croatie), et la construction prévue d'une série de petits barrages. Les espèces envahissantes menacent également les espèces de ce site, avec au moins dix espèces de poissons exotiques et un nombre inconnu de plantes et d'invertébrés exotiques déjà observés. La priorité de cette ZCB est de mettre en œuvre des mesures de gestion à l'échelle du bassin versant, en se concentrant principalement sur la réduction au minimum des perturbations hydrologiques du site, et de maîtriser la propagation des espèces exotiques envahissantes. La gestion du site est particulièrement importante dans la zone du bassin versant située en BosnieHerzégovine, dans laquelle il n'existe aucune mesure de protection. La zone du site située en Croatie est protégée mais l'application de la loi est faible et en grande partie inefficace. La gestion de la truite à lèvres molles (Salmo obtusirostris), qui est une espèce restreinte à seulement quatre lieux (l'un d'entre eux étant le poljé d'Imotski), est aussi une priorité car cette espèce est actuellement menacée par la surpêche et l'hybridation avec les espèces de truites introduites.

Plus au sud, dans le corridor du sud-ouest des Balkans, les lacs Skadar, Ohrid et Prespa sont tous ces sites AZE d'eau douce. La ZCB du lac Skadar [nom anglais : Lake Skadar] est un vaste bassin lacustre méditerranéen comprenant des sources, des cours d'eau et des rivières karstiques ainsi que des marais inondés de manière saisonnière. En lui-même, le lac est un parc national, un site Ramsar et une Zone importante pour la conservation des oiseaux (ZICO). Toutefois, les sources, rivières et cours d'eau associés se situent à l'extérieur de toutes ces aires protégées, rendant ces derniers (et donc le lac) vulnérables aux nombreux impacts extérieurs. Pour cette raison, les limites géographiques de la ZCB s'étendent au-delà des limites du lac lui-même pour inclure les rivières, les sources et les cours d'eau qui se jettent dans le lac, jusqu'aux bassins versants supérieurs des rivières Moraca et Zeta. Le site abrite 24 espèces de déclenchement de ZCB, dont

Lac Skadar (Albanie et Monténégro). ๑ Geert De Knijf. Ce vaste lac méditerranéen, ainsi que son bassin versant associé, est une Zone clé pour la biodiversité (ZCB) d'eau douce, abritant au moins 24 espèces d'eau douce menacées ou ayant une aire de répartition restreinte, comme le Ninnigobius montenegrensis. ๑ Jörg Freyhof 
sept espèces de mollusques endémiques et hautement menacés, en raison desquelles le site répond aux critères d'admission en tant que site AZE. Les principales menaces pesant sur le lac et son bassin versant incluent la pêche potentiellement non durable, les impacts importants au niveau local de la déforestation, de l'extraction du gravier (surtout dans le cours inférieur de la Moraca), du captage excessif de l'eau (préoccupation de grande envergure), et des espèces exotiques envahissantes. Selon les prévisions, les impacts hydrologiques d'un certain nombre de grands barrages prévus devraient, s'ils sont effectivement construits, se traduire par des répercussions sur la biodiversité de cette ZCB unique. Les recommandations en matière de gestion comprennent la mise en œuvre effective de plans d'action pour les salmonidés autochtones, dont certains semblent être proches de l'extinction au niveau local (comme Salmo obtrusirostris), des améliorations générales pour la gestion de la pêche car, selon les observations, la pêche illégale est largement répandue, et une campagne à grande échelle pour sensibiliser à la biodiversité unique de ce site.

La ZCB du lac Ohrid [nom anglais : Lake Ohrid] abrite 68 espèces de déclenchement de ZCB, composées de poissons et de mollusques, rendant cette ZCB l'un des plus importants sites de biodiversité d'eau douce en Europe. Sur ces 68 espèces, 25 sont propres au lac et hautement menacées, faisant de ce lieu un site AZE important. Le lac Ohrid est un site Ramsar et il est considéré que les limites géographiques de la ZCB correspondent aux rives du lac afin de coïncider avec le site Ramsar en tant que tel. Les menaces et les activités de gestion proposées pour le lac n'ont pas encore été identifiées dans le cadre de ce projet donc cela reste une priorité.

Les deux lacs Prespa (grand lac et petit lac) abritent 21 espèces de déclenchement de ZCB. Les limites géographiques de la ZCB coïncident avec celles du parc transfrontalier de Prespa, un exemple de coopération transfrontalière mise en place en 2000 dans le cadre d'une déclaration commune des Premiers ministres des trois pays concernés et qui a été suivie par la signature d'un Accord international pour la protection et le développement durable de la zone du parc de Prespa en 2010 (Albanie, Grèce, ancienne République yougoslave de Macédoine et Commission européenne). Le parc vise à protéger les valeurs écologiques de la zone grâce à la collaboration entre les trois États, et à promouvoir la prospérité économique des communautés locales de ces trois pays. II s'agit de la première aire protégée transfrontalière située dans les Balkans et elle est à présent validée en tant que ZCB d'eau douce couvrant une zone partagée par la Grèce, l'Albanie et l'ancienne République yougoslave de Macédoine (ARYM). Elle comprend deux lacs (le grand lac et le petit lac Prespa) ainsi que leurs bassins versants associés qui s'étendent jusqu'aux sommets montagneux adjacents. Toutefois, la caractéristique la plus importante de l'aire de Prespa est la forte concentration des espèces dans une zone géographique de si petite taille. L'ampleur de cette diversité s'illustre par le fait que neuf des 23 espèces de poissons vivant dans ses lacs et cours d'eau sont endémiques à la $\mathrm{ZCB}$, et par un très haut niveau d'endémisme avec plus de 30 formes endémiques d'organismes lacustres, principalement des gastéropodes, des diatomées, des oligochètes, des sangsues, des spongiaires, des triclades et des ostracodes.

La Grèce abrite aussi de nombreuses ZCB d'eau douce dont 15 répondent aux critères d'admission en tant que sites $A Z E$.
En Grèce, un grand nombre de bassins fluviaux et d'habitats aquatiques sont mal inventoriés concernant leur biote d'eau douce, c'est pourquoi les données sur la répartition des espèces ne sont pas à jour ou disponibles. Toutefois, il existe de nombreux sites de très petite taille connus pour leur importance en termes d'espèces d'eau douce, et ces microhabitats aquatiques représentent des objectifs de conservation importants. Par exemple, la ZCB d'Euboea Manikiatis, le bassin versant d'un cours d'eau isolé sur l'île d'Eubée en Grèce, est aussi désignée en tant que site AZE en raison de deux espèces de poissons endémiques (Barbus euboicus et Squalius sp. «Evia ») et d'une espèce de mollusques endémique (Pseudobithynia euboeensis). $B$. eubiocus est restreint à une unique rivière protégée par une loi grecque qui aide à maintenir le niveau de l'eau. De plus, la présence de $P$. euboeensis est observée uniquement dans quelques petites rivières prenant leur source près de Marmari, à proximité de Karystos. Le captage excessif de l'eau représente une grande menace pour la survie de ces espèces, donc la gestion des ressources en eau dans les bassins versants associés est encore une fois prioritaire.

En conclusion, la sous-région des Balkans abrite certains des écosystèmes d'eau douce ayant la plus forte biodiversité et faisant partie des plus lourdement menacés au sein du hotspot méditerranéen. Une étude sur les espèces menacées parmi les mollusques et poissons d'eau douce de la région (Freyhof 2012) conclut en déclarant que « (...) les Balkans sont le plus important «hotspot» pour la biodiversité d'eau douce menacée en Europe et donc au sein du hotspot de la biodiversité du bassin méditerranéen » [notre traduction]. J. Freyhof indique également qu'« [e]nviron $75 \%$ de tous les poissons menacés et $70 \%$ de tous les mollusques menacés dans les Balkans sont hautement vulnérables à la construction de barrages, aux systèmes d'irrigation et aux travaux hydrologiques, ainsi qu'aux altérations des habitats découlant de ces activités. Ils sont également vulnérables aux invasions d'espèces exotiques, ce qui est un effet secondaire inévitable de la construction de réservoirs". Beaucoup de ces espèces d'eau douce sont endémiques à la région, donc le risque d'extinction à l'échelle mondiale est élevé. La vulnérabilité des mollusques aux perturbations hydrologiques est particulièrement forte car la plupart des espèces menacées sont restreintes à des grottes et à des sources présentes dans les systèmes karstiques. Le drainage et la régulation des poljés karstiques, les transferts d'eau des eaux souterraines vers des tunnels artificiels, et les modifications relatives à la quantité et à la qualité de l'eau ont tous des répercussions sur ces espèces sensibles. En particulier, tous les systèmes d'eaux souterraines et de sources sont menacés par des projets de grande envergure portant sur la régulation de l'eau, comme dans les bassins versants du Neretva-Trebišnjica et de la rivière Cetina. Une grande proportion des mollusques menacés appartiennent à la famille des hydrobiidés, de petits escargots présents quasi exclusivement dans les sources et les systèmes d'eaux souterraines. Les mollusques d'eau douce des lacs Skadar, Prespa et Ohrid sont particulièrement importants car la plupart d'entre eux sont endémiques et également menacés. Pour conserver ces espèces sensibles, la quantité et la qualité de l'eau de ces lacs doivent être préservées et surveillées continuellement. Concernant les poissons d'eau douce, les espèces migratoires sont soumises à un risque particulier car elles ont besoin de longs cours d'eau accessibles sans entrave, 
avec une quantité et un débit d'eau suffisants. Des passes à poissons, si elles sont construites de manière adaptée, peuvent atténuer les problèmes mais en réalité, de nombreux cours d'eau sont équipés de barrages et peu d'entre eux sont dotés de passes efficaces. Les impacts potentiels des espèces exotiques envahissantes sont préoccupants en raison de l'aire de répartition très restreinte d'un grand nombre de ces espèces. Par exemple, il est présumé que la transformation de la ZCB du lac Busko [nom anglais : Lake Busko] en site de pêche récréative, avec introduction de plusieurs espèces appréciées des pêcheurs à la ligne, a probablement déjà fait disparaître plusieurs espèces autochtones du lac (Freyhof, comm. pers.).

La liaison entre le bassin versant de la Moraca et le Danube, qui avait été prévue afin d'accroître l'alimentation en eau des générateurs hydroélectriques, serait susceptible d'avoir de graves répercussions sur de nombreuses espèces de poissons autochtones. Des transferts similaires entre bassins dans de nombreuses autres parties de la région risquent également d'affecter d'autres espèces autochtones avec l'arrivée d'autres espèces autochtones en tant qu'espèces exotiques dans cette zone.

Même si les espèces menacées ou présentant des niveaux d'endémisme élevés sont surtout des poissons et des mollusques, la protection au sein des ZCB peut également être bénéfique à quelques espèces d'odonates (libellules et demoiselles) et de plantes aquatiques. La plupart des espèces de déclenchement parmi les odonates vivent dans les îles grecques comme la Crète et Corfou. La Nymphe de
Grèce (Pyrrhosoma elisabethae)(En danger critique), est une demoiselle faisant partie de ces espèces et est restreinte à l'île grecque de Kérkira, au Péloponnèse, au nord-ouest de la Grèce et au sud de l'Albanie. Cette espèce dépend fortement des petits ruisseaux qui sont souvent détruits lors de travaux d'irrigation, et elle est présente au sein d'un certain nombre de ZCB d'eau douce validées. Les quelques espèces de déclenchement composées de plantes sont également surtout présentes dans les îles grecques. Callitriche pulchra (En danger critique) est l'une des espèces de plantes qui pourrait clairement bénéficier d'une protection du site, car sa présence n'a été observée avec certitude que dans un seul étang sur l'île de Gavdos (au large de la Crète), dans lequel elle est exposée à des risques en raison de l'utilisation intense des réserves présentes dans ce bassin et de l'impact du tourisme. Cette espèce relève de la ZCB du sudouest de la Crête [nom anglais : Crete South-west].

Enfin, il est important de souligner à nouveau que certaines zones de la sous-région des Balkans sont très mal inventoriées concernant leur biote aquatique ; un exemple type est l'Albanie, qui possède peu de sites désignés en comparaison avec la côte ionienne plus au sud. De nombreux projets de développement (comme les barrages) menacent les régimes de débit des cours d'eau et des zones humides en Albanie où le biote aquatique n'est pas assez inventorié. Le manque d'informations sur les espèces des zones humides dans de nombreuses parties de la région représente une grave lacune, laissant de côté de nombreux sites potentiellement importants pour leur biodiversité et risquant d'être affectés par les projets de développement.

Le parc naturel Hutovo Blato, qui comprend un lac et des zones marécageuses, fait partie de la ZCB du cours supérieur et moyen du fleuve Neretva [nom anglais : Lower and Middle Neretva]. Ce site, qui est aussi reconnu en tant que Zone importante pour la conservation des oiseaux (ZICO), abrite de nombreux mollusques et poissons appartenant à des espèces d'eau douce menacées et ayant une aire de répartition restreinte. ๑ Geert De Knijf

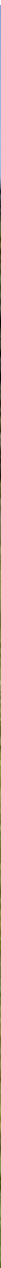


Même si les menaces pesant sur la biodiversité d'eau douce dans cette région sont nombreuses, les bassins versants des Balkans restent relativement intacts en comparaison avec d'autres parties d'Europe où très peu de cours d'eau peuvent être considérés comme ayant un statut de conservation satisfaisant. Ainsi, il est important d'entretenir et d'améliorer les cours d'eau et les lacs de cette région, d'autant plus que la restauration des systèmes dégradés est extrêmement coûteuse et intervient souvent trop tard pour les espèces qui y vivaient autrefois.

\section{Les ZCB d'eau douce dans la sous-région de la Turquie et du Levant}

La sous-région de la Turquie et du Levant inclut le sud et l'ouest de la Turquie, une grande partie de la Syrie, le Liban, le nord de l'Irak, la Jordanie, les Territoires palestiniens occupés et Israël (Figure 11). La région est caractérisée par un certain nombre d'écorégions d'eau douce dont : la Thrace, l'Anatolie occidentale, l'Anatolie méridionale, l'Anatolie centrale, le cours supérieur du Tigre et de l'Euphrate, l'Oronte, la zone côtière du Levant et le Jourdain (Abell et al. 2008). Les corridors prioritaires définis par le CEPF couvrent la majeure partie du hotspot au sein de cette sous-région, et incluent les corridors suivants : Bassin de la mer de Marmara ; Monts Taurus ; Mésopotamie septentrionale ;
Vallée de l'Oronte et monts Liban ; Sud de la Syrie et nord de la Jordanie. La synthèse suivante repose en grande partie sur les informations obtenues en ligne à partir du site Freshwater Ecoregions of the World (www.feow.org).

L'Anatolie occidentale comprend la rivière Dalaman qui sillonne une gorge escarpée et profonde, contrairement aux cours d'eau méandriques typiques de la côte égéenne (comme le Büyük Menderes et le Gediz). La partie de l'Anatolie centrale se trouvant dans le hotspot comprend une multitude de bassins endoréique sur le plateau d'Anatolie, incluant les lacs Eğirdir, Burdur et Aci. La plupart des cours d'eau de l'Anatolie méridionale prennent leur source dans les monts Taurus et se jettent dans la mer Méditerranée. Le delta du Göksu (ou Gök) et la lagune d'Akyatan près d'Adana sont des sites Ramsar. L'écorégion du cours supérieur du Tigre et de l'Euphrate est essentiellement composée des sections supérieures de ces deux fleuves, où sont présents quelques lacs de petite taille mais aucun grand marais ou habitat lacustre naturel comme ceux observés dans l'écorégion du cours inférieur du Tigre et de l'Euphrate. L'écorégion d'Oronte inclut le fleuve Oronte, les cours d'eau Afrin et Karasu dont la source est au nord, et l'ancien lac Amik. Autrefois le lac Amik était un habitat d'eau douce important, surtout pour les oiseaux d'eau migratoires, ce qui était relativement inhabituel pour le sudouest de l'Asie, mais il a été drainé entre les années 1940 et 1970 afin de libérer des terres pour la culture du coton, d'éradiquer la malaria et de construire un aéroport.

L'écorégion de la zone côtière du Levant englobe le littoral du Levant allant du versant occidental des monts Jabal an Nusayriyah

Figure 11. La sous-région de la Turquie et du Levant.

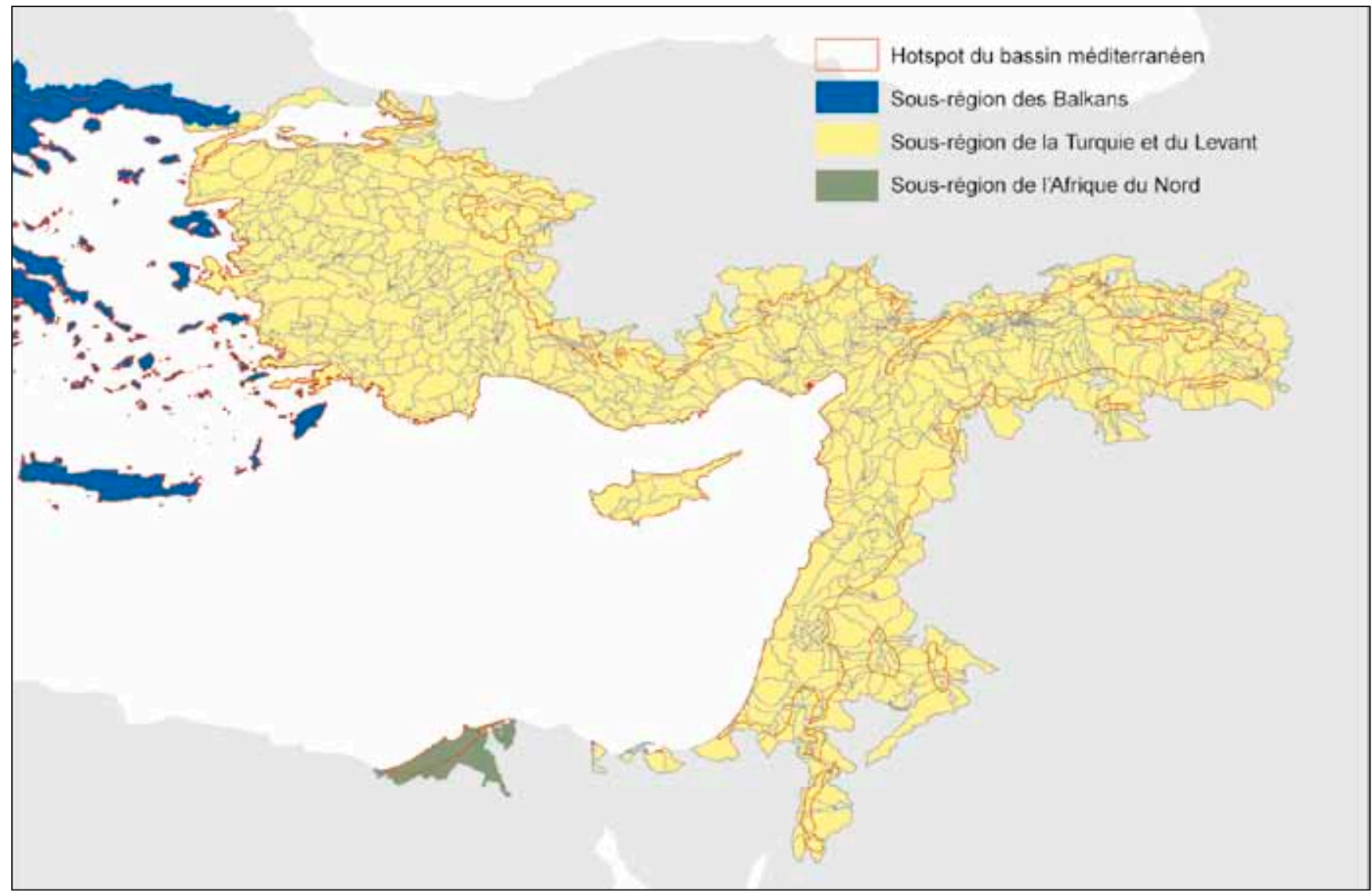




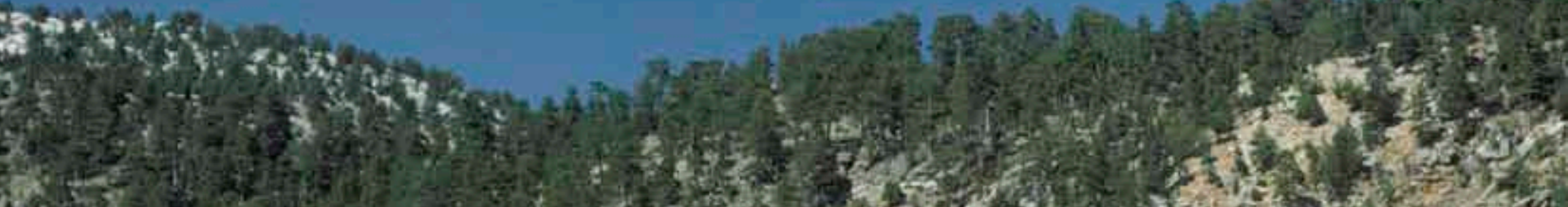

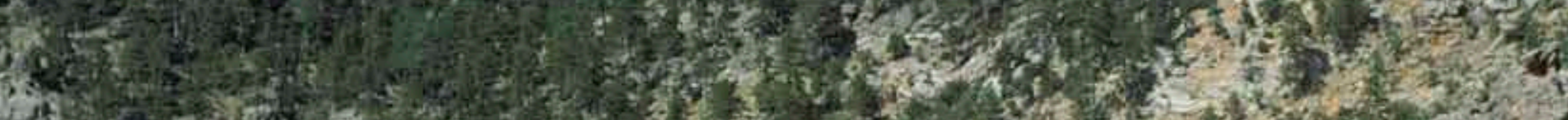

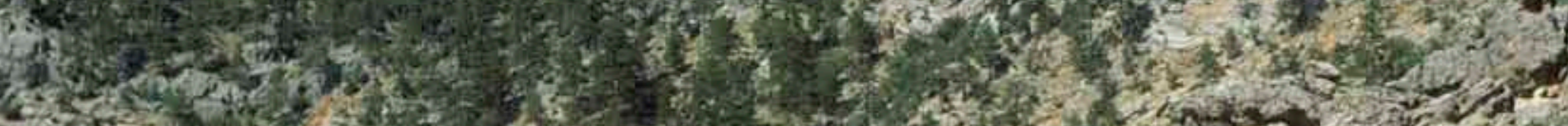

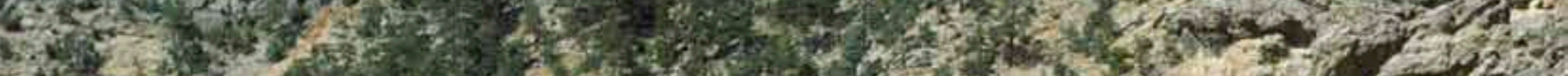

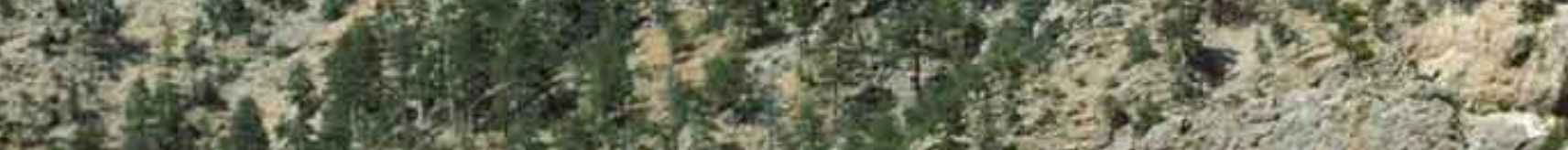

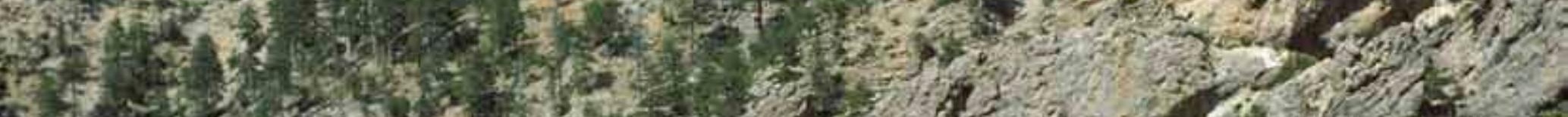

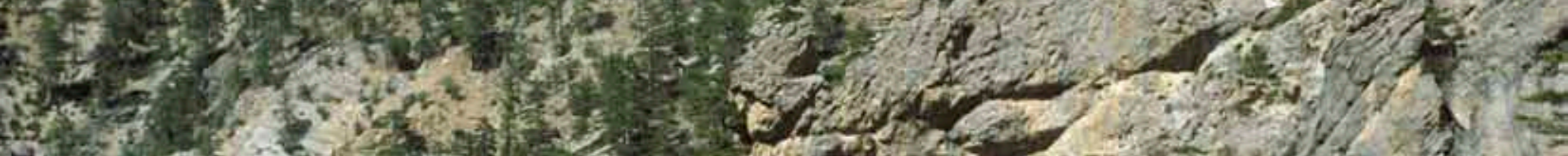

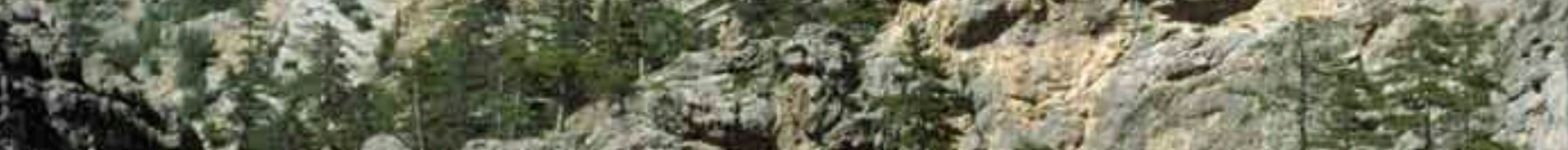

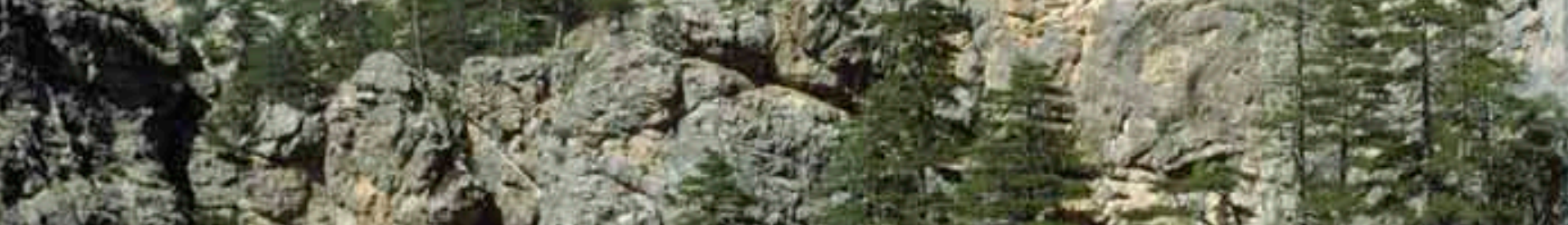

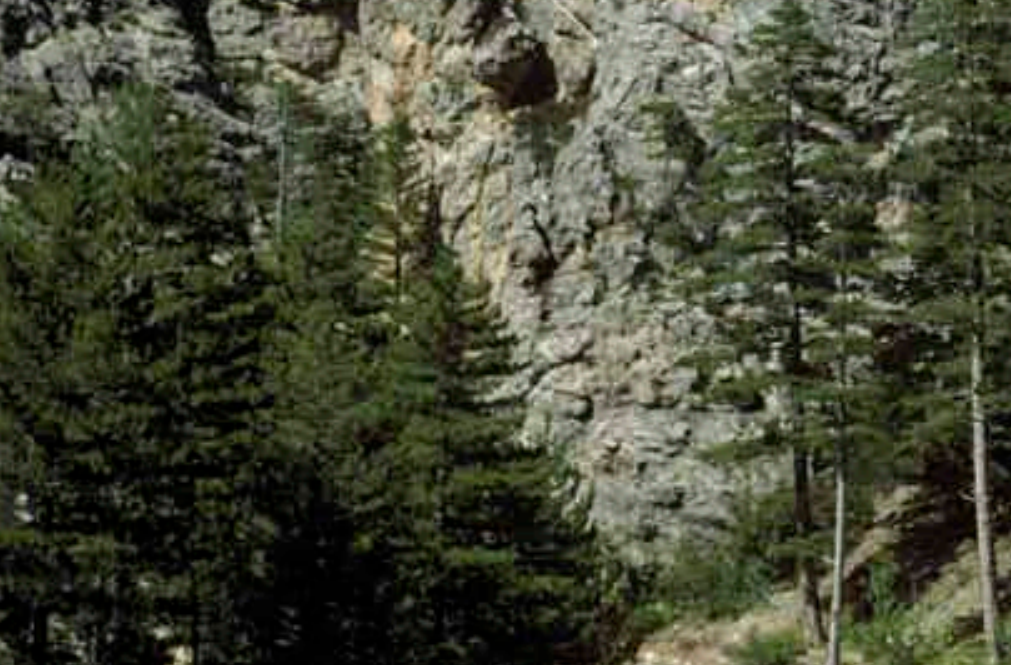

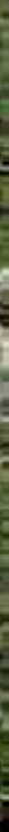


en Syrie, des monts Liban au Liban et des collines de Judée en Israël, jusqu'au Sinaï. Elle couvre une étroite plaine côtière s'appuyant sur des chaînes montagneuses. Les rivières côtières s'écoulant du mont Liban vers la mer Méditerranée sont courtes et profondes, certaines s'asséchant en été. Le fleuve Litani (fortement pollué) chemine parallèlement à la côte avant de virer brusquement vers l'ouest pour traverser la chaîne montagneuse côtière et se jeter dans la mer. La zone marécageuse nommée Aammiq (sans rapport avec le lac Amiq de l'Oronte) sur le fleuve Litani a été récupérée pour l'agriculture, et de grandes parties du marais restant s'assèchent en été. Le principal cours d'eau de l'écorégion de la Jordanie est le Jourdain, un fleuve qui prend sa source dans la chaîne montagneuse de l'Anti-Liban. II relie la mer de Galilée (lac de Tibériade ou lac de Kinnereth) à la mer Morte mais aujourd'hui, en raison d'un détournement de son cours, il s'agit plutôt d'une rivière. Les oueds (vallées dotées de cours d'eau intermittents) se jetant dans le Jourdain incluent les oueds Mujib, Mousa, Hassa et Zarqa. L'écorégion abrite des espèces reliques téthysiennes, dont des crevettes aveugles, des mollusques souterrains et des éponges endémiques.

\section{Aperçu des ZCB}

Pour la sous-région de la Turquie et du Levant, nous avons étudié 872 sous-bassins versants (résolution HydroBASINS de niveau 8), couvrant une superficie de $508351 \mathrm{~km}^{2}$. Au sein de ces sous-bassins versants, 608 ZCB proposées ont été identifiées en tant que sous-bassins versants remplissant les critères de ZCB en raison des groupes taxonomiques d'eau douce évalués à cet endroit (Figure 12, Tableau 7). Concernant les espèces de déclenchement de ZCB, 165 espèces de ce type ont été identifiées. Une consultation ultérieure auprès des parties
Tableau 7. Synthèse des ZCB proposées. Nombre de sous-bassins versants proposés en tant que ZCB en raison de la présence d'espèces menacées (C1), d'espèces ayant une aire de répartition restreinte (C2), et de communautés restreintes à un biome (C3) pour chaque groupe taxonomique. À noter : les chiffres de la colonne "Tous les critères " représentent le nombre total de bassins versants distincts qualifiés, et ne correspondent donc pas à la somme des lignes.

\begin{tabular}{|lcccc|}
\hline & \multicolumn{4}{c}{ Nombre de sous-bassins versants qualifiés } \\
\hline & Tous les critères & C1 & C2 & C3 \\
\hline Poissons & 413 & 320 & 205 & 16 \\
\hline Mollusques & 583 & 580 & 42 & 3 \\
\hline Odonates & 160 & 130 & 98 & 0 \\
\hline Plantes & 25 & 24 & 20 & 4 \\
\hline Total & $\mathbf{6 0 8}$ & $\mathbf{6 0 7}$ & $\mathbf{2 9 6}$ & $\mathbf{2 3}$ \\
\hline
\end{tabular}

prenantes concernant ces sous-bassins versants a conduit à la désignation et à la délimitation de 47 ZCB validées (Figure 13, Tableau 8). Ainsi, 122 espèces remplissant au moins l'un des critères de ZCB ont été identifiées (Annexe IV, Tableau C). Le nom des ZCB et le nombre d'espèces de déclenchement sont indiqués dans le Tableau 9.

\section{Niveaux actuels de protection}

La zone des ZCB d'eau douce validées se trouvant dans les limites géographiques des aires protégées (AP) existantes et des autres ZCB représente $32172 \mathrm{~km}^{2}$ (24\% de la superficie totale des ZCB d'eau douce validées dans la sous-région de la Turquie et du Levant). Ceci signifie que $76 \%$ de la superficie de ces ZCB d'eau douce se situent à l'extérieur des aires protégées officielles

Figure 12. Ensemble des ZCB d'eau douce proposées avec indication du nombre d'espèces de déclenchement de ZCB dans la sous-région de la Turquie et du Levant.

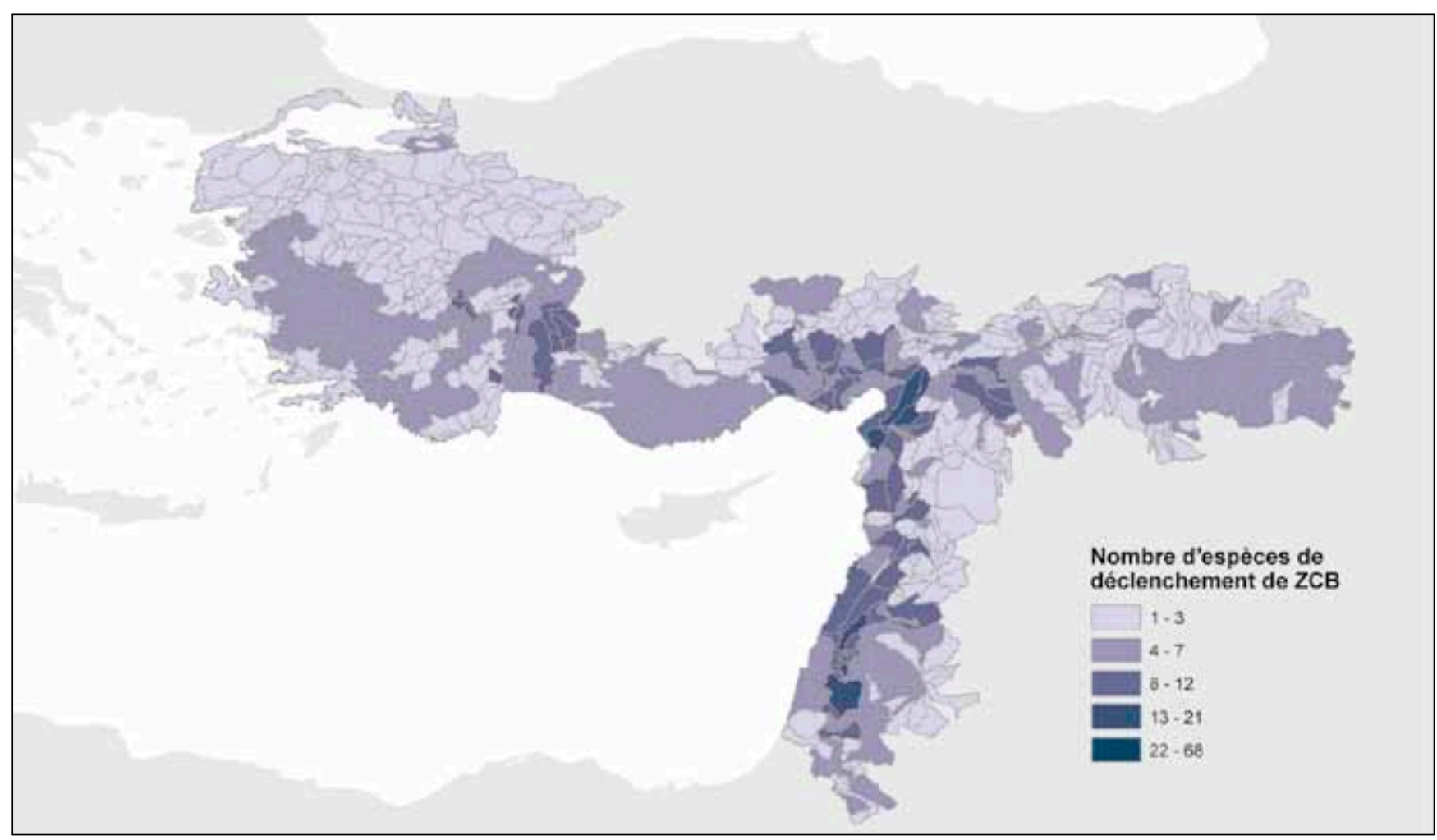




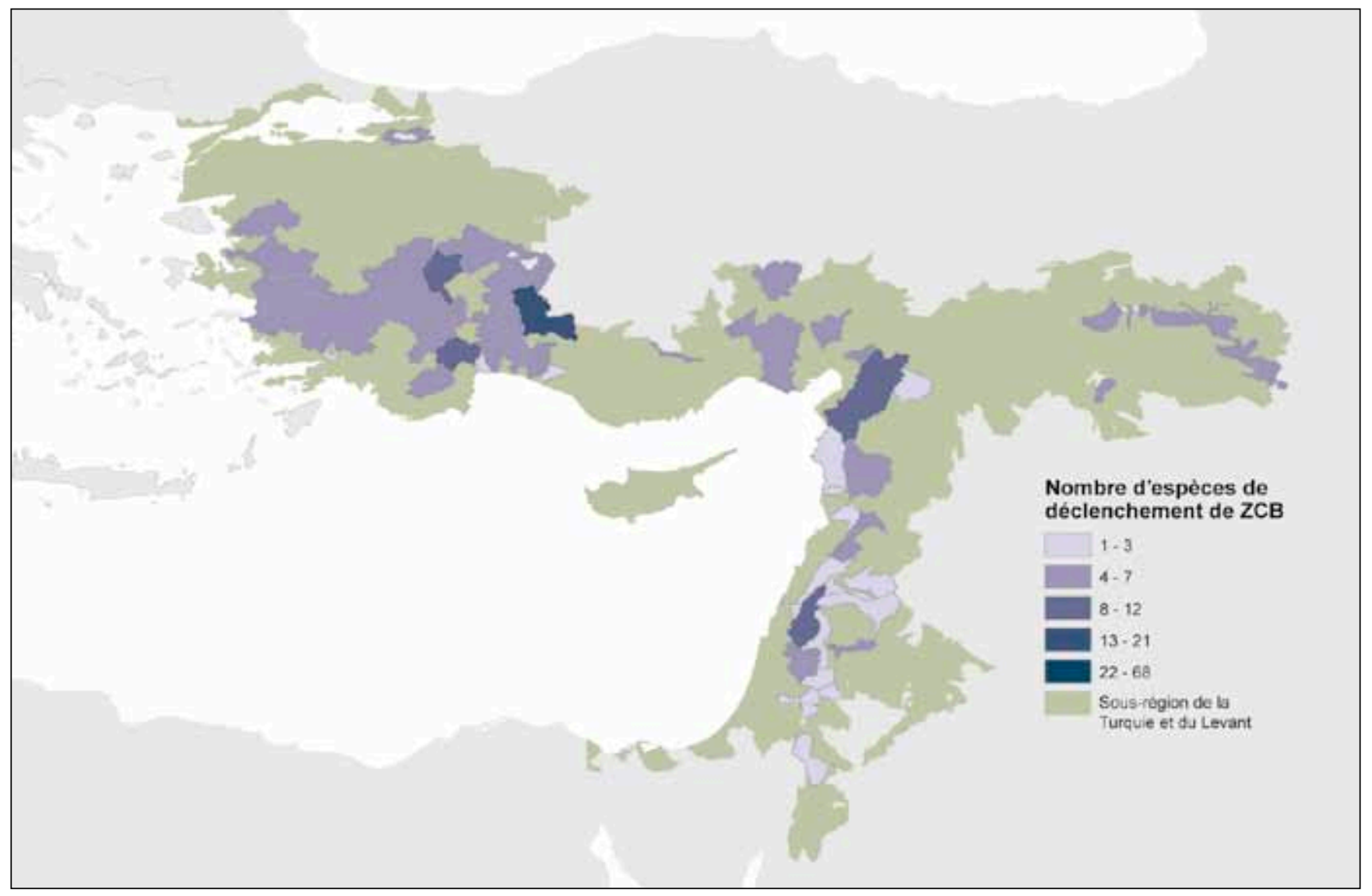

Figure 13. Ensemble des ZCB validées avec indication du nombre d'espèces de déclenchement de ZCB dans la sous-région de la Turquie et du Levant.

La ZCB de la source de Barada [nom anglais : Spring of Barada (En Fidje)] en Syrie. Cette source a été drainée quasi intégralement en 2008 pour répondre aux besoins croissants en eau. Par conséquent, le poisson endémique Pseudophoxinus syriacus (CR) (voir encadré) est maintenant Peut-être Éteint. @ Jörg Freyhof

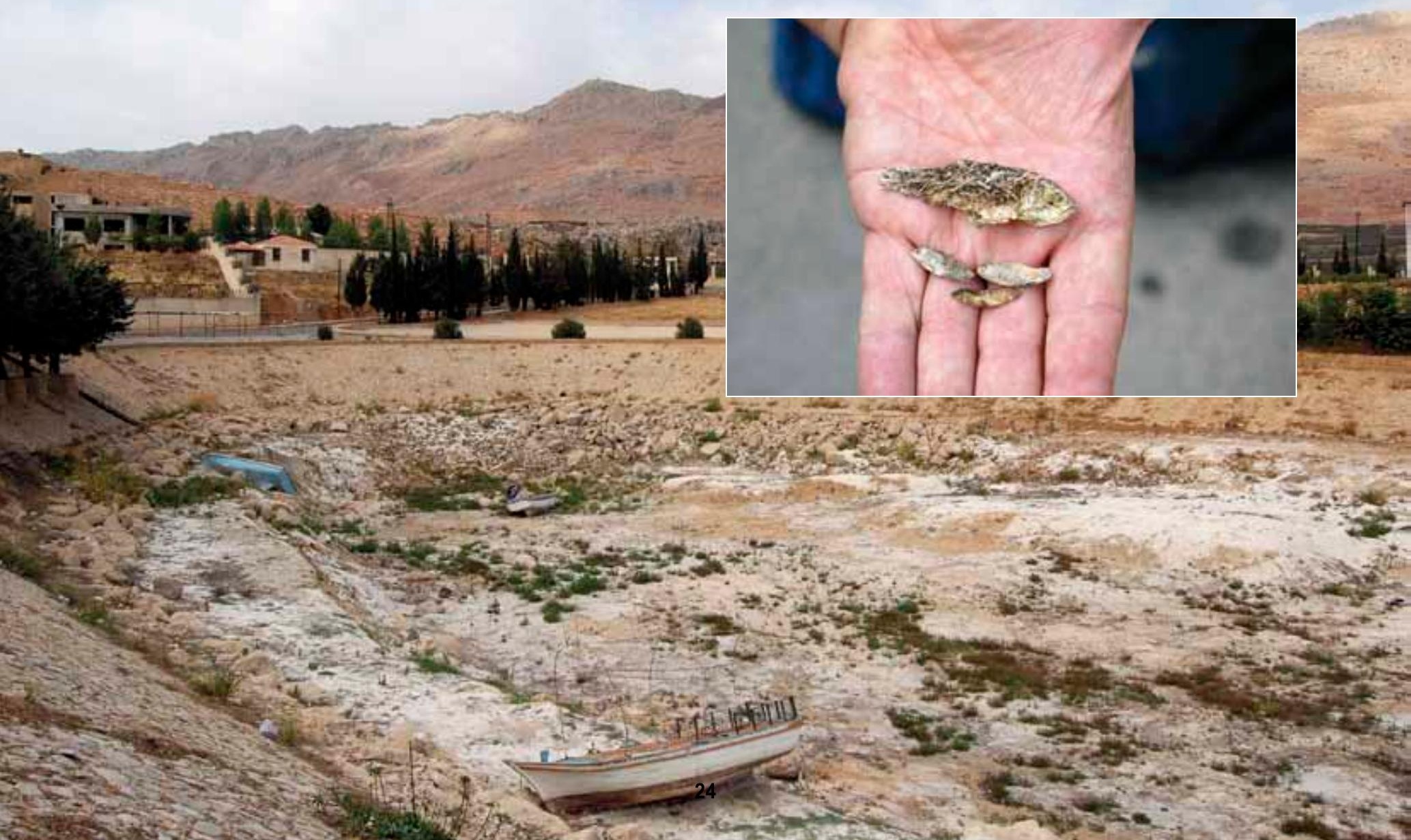




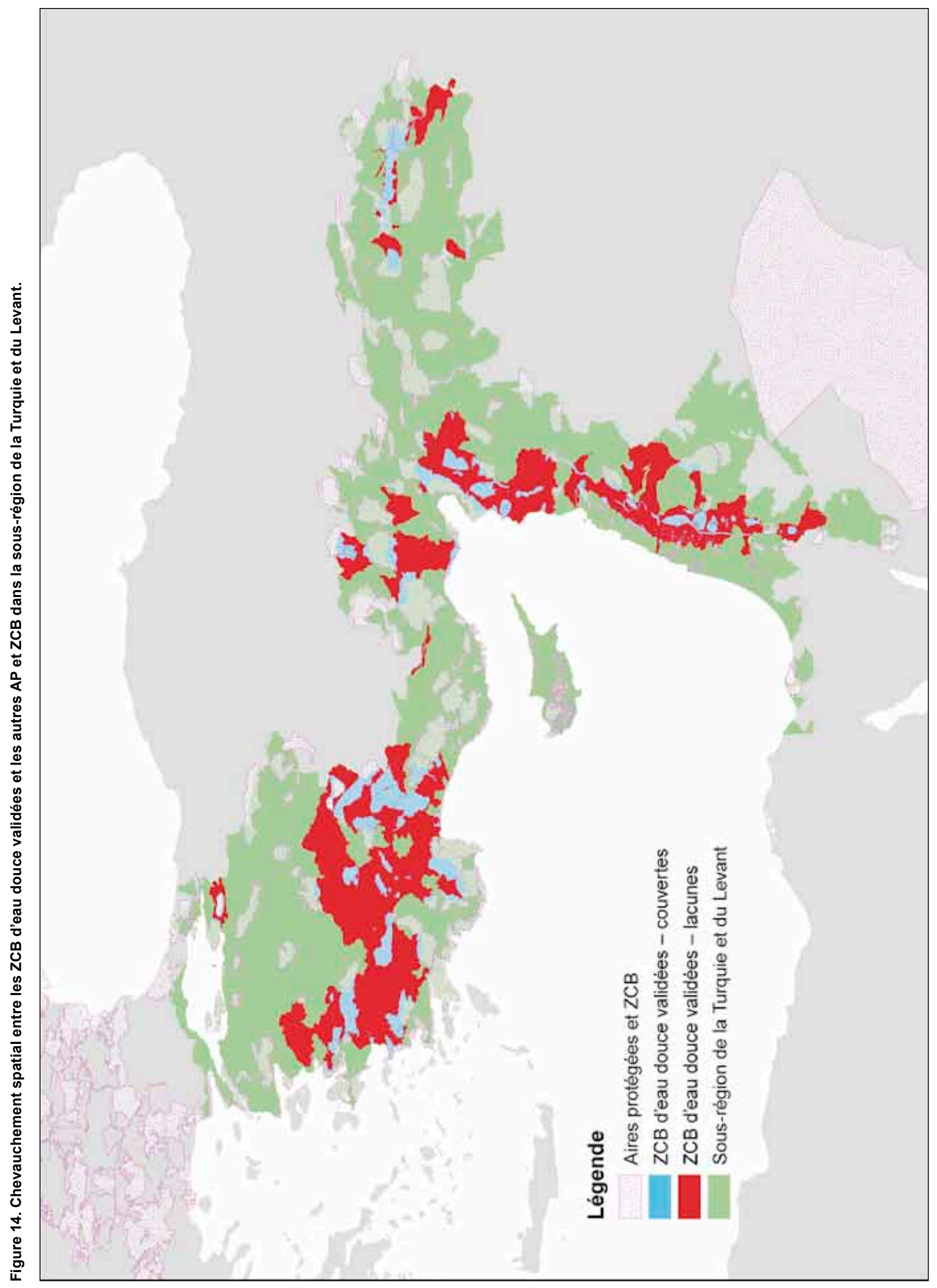


Tableau 8. Synthèse des ZCB validées. Nombre de sous-bassins versants validés en raison de la présence d'espèces menacées (C1), d'espèces ayant une aire de répartition restreinte (C2), et de communautés restreintes à un biome (C3) pour chaque groupe taxonomique. À noter : les chiffres de la colonne "Tous les critères " représentent le nombre total de bassins versants distincts qualifiés, et ne correspondent donc pas à la somme des lignes. Concernant cette sous-région, les sites abritant des plantes aquatiques et des plantes de milieux humides, ainsi que des odonates (dont le nombre est bien inférieur à celui des poissons et des mollusques), n'ont pas pu être inclus dans le cadre des ateliers des parties prenantes. Les ZCB proposées en raison de ces deux regroupements taxonomiques sont présentées à l'Annexe VI et sont en attente de validation de la part des parties prenantes.

\section{Nombre de sous-bassins versants qualifiés}

\begin{tabular}{|lcccc|} 
& \multicolumn{4}{c}{ Nombre de sous-bassins versants qualifiés } \\
\hline & Tous les critères & C1 & C2 & C3 \\
\hline Poissons & 45 & 43 & 35 & 4 \\
\hline Mollusques & 39 & 36 & 26 & 3 \\
\hline Odonates & n/a & n/a & n/a & n/a \\
\hline Plantes & n/a & n/a & n/a & n/a \\
\hline Total & $\mathbf{4 7}$ & $\mathbf{4 7}$ & $\mathbf{4 1}$ & $\mathbf{7}$ \\
\hline
\end{tabular}

ou d'autres sites reconnus pour leur importance biologique en tant que ZCB (Figure 14). La seule ZCB d'eau douce validée ne faisant l'objet d'aucune couverture par une autre ZCB ou AP est Amman.

\section{Discussion}

La sous-région de la Turquie et du Levant abrite un certain nombre de systèmes fluviaux, lacustres et karstiques, et de systèmes de zones humides importants qui ont été validés en tant que ZCB d'eau douce, dont 18 sont aussi des sites AZE (y compris deux qui le sont potentiellement) (Tableau 9). Les principales menaces pesant sur les ZCB d'eau douce de la région comprennent : i) les impacts hydrologiques de la construction de barrages et le captage excessif des eaux souterraines et de surface ; ii) la perte et la dégradation des terres lorsqu'elles sont transformées pour l'agriculture ; iii) les espèces exotiques envahissantes ; iv) la surpêche, et ; v) la pollution de l'eau. Contrairement à certaines parties de la Méditerranée occidentale, de nombreux habitats aquatiques de la Méditerranée orientale sont peu étudiés par les

Tableau 9. Liste des ZCB d'eau douce validées pour les bassins versants de la sous-région de la Turquie et du Levant, indiquant le nombre d'espèces de déclenchement de ZCB, les sites AZE (*) et, si elles ont été identifiées, les Zones focales pour chaque ZCB. Les ZCB transfrontalières figurent à la fin du tableau.

\begin{tabular}{|c|c|c|c|}
\hline Pays & Nom de la ZCB ${ }^{1}$ & $\begin{array}{c}\text { Nombre } \\
\text { d'espèces de } \\
\text { déclenchement }\end{array}$ & Zones focales \\
\hline Jordanie & Amman & 2 & \\
\hline Jordanie & Upper Mujib* & 2 & Sources près de la ville de Swaymeh - Beer AI Azraq \\
\hline Jordanie & Wadi Shuaib & 2 & Partie occidentale du bassin versant \\
\hline Jordanie & Zarqa River & 3 & Source de Rumeimin \\
\hline Syrie & Middle Orontes & 4 & $\begin{array}{l}\text { Deux zones focales pour } M \text {. dircaena dans le bassin versant et } \\
\text { Syrofontana qui est endémique du Gab dans le bassin versant }\end{array}$ \\
\hline Syrie & Nahr al Aouaj & 3 & Partie supérieure (occidentale) \\
\hline Syrie & Nahr al Marqiya* & 1 & \\
\hline Syrie & Upper Khabour & 7 & Sources de Ras Al Ain (pour les mollusques) \\
\hline Turquie & Aksu River & 5 & Section inférieure du cours d'eau \\
\hline Turquie & Akyaka springs* & 2 & Sources du cours supérieur \\
\hline Turquie & Bakirçay & 6 & Deux rivières dans le Bakir \\
\hline Turquie & Burdur lake and catchments* & 6 & Lac Burdur, lac Karatas, rivière Duger \\
\hline Turquie & Büyük Menderes river & 7 & Rivière Kamis \\
\hline Turquie & Düden River* & 2 & \\
\hline Turquie & Eğirdir Lake catchment* & 7 & Rivière Çaykoy \\
\hline Turquie & Gökdere (Yeşildere) stream* & 4 & \\
\hline Turquie & Işıklı/Çivril lake and catchment* & 8 & Sources situées dans les affluents du fleuve Büyük Menderes \\
\hline Turquie & Karpuzçay stream* & 3 & Source de Murtici \\
\hline Turquie & Kopru Çay* & 5 & \\
\hline Turquie & Korkuteli and Elmali plains & 4 & Lac d'Avlan avec vestiges du lac Sogut et de Karagol \\
\hline Turquie & Küçük Menderes & 5 & Delta côtier d'eau douce (375'24" $\left.27^{\circ} 17^{\prime} 30.81^{\prime \prime}\right)$ \\
\hline Turquie & Lake Beyşehir and catchments* & 13 & Parties inférieure et moyenne au nord \\
\hline Turquie & Lake Iznik and catchment* & 5 & $\begin{array}{l}\text { Zone focale de la source de Cakirca pour les poissons, et source de } \\
\text { Pinarbasi }\end{array}$ \\
\hline Turquie & Lakes Acıgöl and Salda & 5 & $\begin{array}{l}\text { Zone des sources d'eau douce autour des rives du lac Acıgöl et du } \\
\text { lac Yarishli, et lac Saldagol lui-même }\end{array}$ \\
\hline Turquie & Lakes Akşehir - Eber system & 5 & Affluents des lacs, en particulier la partie orientale du lac Akşehir \\
\hline Turquie & Lower Gediz river & 6 & $\begin{array}{l}\text { Lac Marmara (abritant d'importantes populations appartenant } \\
\text { aux espèces de poissons de cette région) }\end{array}$ \\
\hline Turquie & Manavgat River & 5 & \\
\hline Turquie & Middle and lower Seyhan river & 6 & \\
\hline Turquie & $\begin{array}{l}\text { Savrun catchment (Ceyhan } \\
\text { drainage) }\end{array}$ & 6 & \\
\hline
\end{tabular}


Tableau 9. Liste des ZCB d'eau douce validées pour les bassins versants de la sous-région de la Turquie et du Levant (suite).

\begin{tabular}{|c|c|c|c|}
\hline Pays & Nom de la ZCB ${ }^{1}$ & $\begin{array}{c}\text { Nombre } \\
\text { d'espèces de } \\
\text { déclenchement }\end{array}$ & Zones focales \\
\hline Turquie & Sultan Sazlığı Marshes* & 5 & Marais/sources et zones humides saisonnières dans leur ensemble \\
\hline Turquie & Upper Dalman & 4 & Sources de Tefenni \\
\hline Turquie & $\begin{array}{l}\text { Yarpuz and Hammus catchment } \\
\text { (in Ceyhan basin) }\end{array}$ & 6 & \\
\hline Jordanie, Israël & Wadi Karak Basin & 2 & Oued Karak Ibnhamad Hadditha \\
\hline $\begin{array}{l}\text { Jordanie, Israël, } \\
\text { Territoires palestiniens } \\
\text { occupés }\end{array}$ & Central Jordan River & 5 & \\
\hline Liban, Syrie & Upper Asi Lebanon* & 6 & \\
\hline Liban, Syrie, Israël & Upper Jordan Valley & 9 & $\begin{array}{l}\text { Mer de Galilée (aussi appelée lac de Tibériade ou lac de Kinnereth) } \\
\text { et sources environnantes et marais de Hula (restaurés) }\end{array}$ \\
\hline $\begin{array}{l}\text { Territoires palestiniens } \\
\text { occupés }\end{array}$ & Jerico catchment & 3 & \\
\hline Syrie, Jordanie & Yarmuk basin ${ }^{*}$ & 6 & Lac Muzayrib \\
\hline Syrie, Jordanie, Israël & Lower Yarmouk & 2 & Zone allant de Maqarin à l'oued Shaq Al-barid \\
\hline Syrie, Liban & Lake Homs (Qatinah) & 7 & Fleuve Oronte et lac Homs \\
\hline Syrie, Liban & Litani River* & 2 & Marais d'Aammiq \\
\hline Syrie, Liban & Nahr al Kabir & 3 & \\
\hline Syrie, Liban & Spring of Barada (En Fidje) & 2 & Flux en sortie de source \\
\hline Turquie, Syrie & Lower Asi drainage & 10 & $\begin{array}{l}\text { Réservoir du bassin Tahta Köprü et ses rivières connexes, lac } \\
\text { Golbasi. Büyük KaraÇay et Küçük KaraÇay }\end{array}$ \\
\hline Turquie, Syrie & $\begin{array}{l}\text { Northern Coastal Streams of } \\
\text { Syria* }\end{array}$ & 2 & $\begin{array}{l}\text { Cours inférieur des rivières et deux petites rivières entre Baniyas } \\
\text { et Jableh }\end{array}$ \\
\hline Turquie, Syrie & Qweik $^{*}$ & 3 & Cours supérieur en Turquie (emplacement des eaux résiduelles) \\
\hline Turquie, Syrie, Irak & Main stem of the Tigris River & 5 & \\
\hline
\end{tabular}

1 N.D.T. : Les noms des sites ZCB figurant dans les tableaux sont en anglais. Toutefois, les noms des sites ZCB cités dans le corps de ce document ont été traduits en français en précisant leur nom anglais entre crochets (" [nom anglais : XXX] ), afin de permettre au lecteur de faire le lien avec les noms des sites en anglais figurant dans les tableaux correspondants et dans la base de données en ligne (WBDB, World Biodiversity DataBase).

scientifiques ; pour cette raison, certaines espèces susceptibles de présenter un intérêt particulier sont peu documentées, et dans certains cas, la confirmation même de leur survie fait défaut, comme l'illustre le cas de la blennie fluviatile (Salaria fluviatilis), une espèce chypriote (Zogaris et al. 2014). Les inventaires des habitats et des espèces, ainsi que d'autres travaux taxonomiques visant à clarifier la validité des taxons, revêtent une importance critique pour la conservation aquatique de cette zone.

Pour plus d'informations sur le statut de la biodiversité d'eau douce et les habitats des zones humides dans l'est de la Méditerranée, voir le rapport complémentaire intitulé Status and Distribution of Freshwater Biodiversity in the Eastern Mediterranean (Smith et al. 2014) qui met en lumière les résultats des évaluations de la Liste rouge de I'UICN. Les difficultés et les recommandations de gestion concernant ces ZCB sont soulignées à nouveau plus bas, dans le cadre de la discussion sur quelques sites sélectionnés.

La ZCB des sources d'Akyaka [nom anglais : Akyaka Springs] dans le sud-ouest de la Turquie est un site AZE englobant les sources et leurs deux courtes rivières permanentes, nommées Akçapınar et Kadın Azmağı. La ZCB répond aux critères d'admission en tant que site AZE en raison de la présence du mollusque Bithynia pesicii (En danger) dont l'aire de répartition est restreinte à cette ZCB. Il est également présumé que la mulette épaisse (Unio crassus), une espèce de moules de rivière En danger, est présente sur ce site. Les principales menaces sont l'enrichissement en nutriments provenant du ruissellement agricole, et les eaux usées issues d'un complexe touristique côtier en expansion à proximité d'Akyaka. Ces deux espèces étant sensibles aux modifications de la qualité de l'eau, le problème actuel de la mauvaise qualité de l'eau doit être réglé. Également en Turquie, la ZCB du bassin versant du lac Eğirdir [nom anglais : Eğirdir Lake catchment] en Anatolie centrale inclut le lac karstique en lui-même ainsi que ses bassins versants, dont un certain nombre de sources. Le site répond aux critères d'admission en tant que site AZE en raison des deux espèces de mollusques endémiques, Bythinella turca (CR) et Falsipyrgula pfeiferi (EN), et d'une troisième espèce éventuelle, Graecoanatolica lacustristurca (EN) (qui est susceptible d'avoir disparu dans deux de ses trois emplacements), et en raison de deux espèces de poissons endémiques, Pseudophoxinus egridiri (EN) et Capoeta pestai (CR). Les principales menaces pesant sur le site sont typiques de la région et comprennent l'eutrophisation du lac, le captage excessif et la pollution de l'eau, les espèces envahissantes et, dans le cas de la source de Kayaagzi, des travaux de construction routière. La présence de $B$. turca n'a été observée que dans une seule source (Cire), au milieu du village, où elle risque de subir les effets de la modification des habitats à des fins récréatives, par exemple en bétonnant le fond de la source principale et par le dégagement des rives. Les espèces $F$. pfeiferi et $G$. lacustristurca sont toutes les deux endémiques au lac Eğirdir où l'impact de l'eutrophisation (le lac est naturellement oligotrophe) et les espèces exotiques envahissantes menacent potentiellement ces espèces. Les espèces de poissons subissent aussi les effets de l'introduction du sandre doré européen (Sander lucioperca) et de Capoeta pestai en raison de la surpêche. En Anatolie méridionale, la ZCB 
du fleuve Düden [nom anglais : Düden River] répond aussi aux critères d'admission en tant que site AZE en raison de la présence du mollusque Hydrobia anatolica (En danger critique), qui n'a été observée que dans la cascade du cours supérieur du Düden. L'espèce de poisson Pseudophoxinus antalyae (Vulnérable) est aussi endémique à la ZCB. La principale menace pesant actuellement sur cette ZCB est le captage excessif de l'eau par la ville d'Antalya à des fins d'irrigation ou pour un usage domestique, ce qui a un impact sur le système d'eaux souterraines karstiques alimentant ces sources et le fleuve en lui-même. Plus à l'est, la ZCB des marais de Sultan Sazlığı [nom anglais : Sultan Sazlığı Marshes], dans la dépression de Develi, associe un ensemble complexe de marais d'eau douce et des mares d'eau saline/saumâtre ainsi que des vasières inondées de manière saisonnière et une végétation halophile. Cette $Z C B$ répond aussi aux critères d'admission en tant que site AZE en raison de la présence de deux espèces de poissons En danger critique, Aphanius danfordii et Pseudophoxinus elizavetae. Une fois de plus, ce site court un risque important en raison du captage des eaux souterraines à des fins d'irrigation. Autrefois, les marais de Sultan Sazlığı représentaient une zone humide très importante mais cette zone a été drainée et a fini par s'assécher au début du $X X \mid{ }^{e}$ siècle. Aujourd'hui, ces deux espèces de poissons survivent dans seulement quelques petites sources et quelques canaux. La ZCB s'étend jusqu'à inclure l'ensemble du bassin versant car tous les plans d'eau sont alimentés à partir d'un seul aquifère. Le drainage continu de cette zone doit être stoppé si l'on veut permettre à ces espèces de survivre.

La ZCB de Nahr al Marqīyah en Syrie est un système karstique côtier. II s'agit aussi d'un site AZE en raison de la présence de Pseudophoxinus hasani, un poisson endémique à la rivière côtière Nahr Marqīyah qui n'est actuellement pas protégée.
La principale menace est à nouveau le captage de l'eau et la rétention d'eau par les barrages. Le développement économique rapide survenant dans cette zone est susceptible de conduire à une augmentation de la demande en eau, c'est pourquoi les flux environnementaux de la rivière doivent être déterminés et respectés si l'on veut permettre à cette espèce de survivre à l'état sauvage. La ZCB du fleuve Litani [nom anglais : Litani River] au Liban associe le système fluvial à des marais alimentés par des sources et reliés hydrologiquement, y compris le marais d'Aammiq qui est la plus vaste zone humide d'eau douce subsistant au Liban, un vestige des marais et des lacs qui étaient jadis bien plus étendus. La ZCB répond aussi aux critères d'admission en tant que site AZE d'eau douce en raison de la présence du poisson Tylognathus festai dans le marais d'Aammiq. D'autres espèces de déclenchement incluent la loche Oxynoemacheilus leontinae (Préoccupation mineure) dont la présence est restreinte aux bassins du Litani et du nord du Jourdain, et l'espèce Potomida littoralis (En danger), une moule de la famille des unionidés, largement répandue en Europe du Sud, en Afrique du Nord et au Moyen-Orient, mais qui diminue à un rythme alarmant dans son aire de répartition hautement fragmentée. Le captage de l'eau et les sécheresses sont considérés comme étant les principales menaces pesant sur l'hydrologie locale et les zones de marais en particulier. Une conservation ex situ est recommandée pour l'espèce $T$. festai en attendant de pouvoir stabiliser et restaurer l'hydrologie de son habitat naturel grâce à une meilleure gestion de l'utilisation de l'eau et à une amélioration des pratiques agricoles. Un dernier exemple est la ZCB du bassin de la rivière Yarmuk [nom anglais : Yarmuk Basin] qui inclut le cours supérieur de la rivière Yarmuk et le lac Muzayrib (la zone focale de cette ZCB) en Syrie. Cette $Z C B$ répond aussi aux critères d'admission en tant que site AZE en raison de la présence du mollusque Melanopsis pachya

Cascade du Düden au sein de la ZCB du fleuve Düden, en Anatolie méridionale. Il s'agit également d'un site AZE car la présence du mollusque Hydrobia anatolica (En danger critique) n'a été observée qu'à cet endroit. @ Zolakoma. Online image/Flickr (CC BY 2.0)

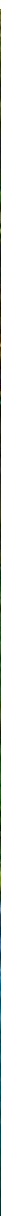




\section{Les ZCB d'eau douce dans la sous-région de l'Afrique du Nord}

La sous-région de l'Afrique du Nord au sein du hotspot inclut les côtes méditerranéennes du Maroc, de l'Algérie, de la Tunisie, de la Libye et de l'Égypte, et comprend les neuf corridors prioritaires définis par le CEPF. À l'exception du delta du Nil, les cours d'eau permanents existent uniquement dans les zones septentrionales du Maroc, de l'Algérie et de la Tunisie, c'est-à-dire dans la région alimentée par les pluies et la fonte des neiges provenant du massif de l'Atlas (Figure 15). La région est caractérisée par les écorégions d'eau douce du Maghreb permanent, du Maghreb temporaire et du delta du Nil (Thieme et al. 2005). Le Maghreb permanent, qui s'étend le long des zones septentrionales du Maroc, de l'Algérie et de la Tunisie, est caractérisé par une faune d'eau douce composée de nombreux éléments européens (Jacquemin et Boudot 1999 ; Samraoui et Menai 1999 ; Jödicke et al. 2000 ; Annani et al. 2012), des reliques afrotropicales (Samraoui et al. 1993) et un endémisme relativement élevé (Samraoui et al. 2012). Les descriptions suivantes s'appuient en grande partie sur celles fournies par Thieme et al. (2005) et par García et al. (2010).

Le Maroc possède le système fluvial le plus étendu en Afrique du Nord. Les précipitations tombant sur les hautes montagnes du Rif, du Moyen Atlas, du Haut Atlas et de l'Anti-Atlas alimentent les cours d'eau qui, en général, coulent dans la direction nord-ouest pour se jeter dans l'océan Atlantique ou bien dans la direction sud-est vers le Sahara. L'oued Moulouya est la principale

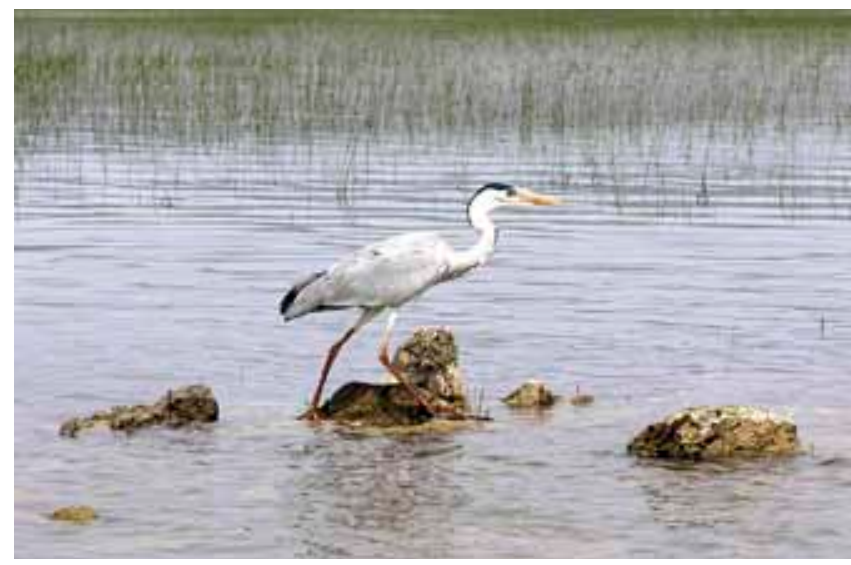

Le lac de Fetzara, appartenant à la ZCB de la Numidie occidentale [nom anglais : Western Numidia], abrite un certain nombre d'espèces de poissons et de plantes aquatiques menacées au niveau mondial. La présence du triton de Poiret (Pleurodeles poireti) (En danger) a également été observée dans cette ZCB. Le lac de Fetzara est aussi un site Ramsar inscrit en raison des nombreux oiseaux d'eau fréquentant ce lieu. @ Samraoui Boudjéma

exception, s'écoulant sur environ $500 \mathrm{~km}$ dans la direction nordest, du Moyen Atlas jusqu'à la mer Méditerranée. Le Maroc possède également un certain nombre de lacs de montagne situés à une altitude supérieure à $1800 \mathrm{~m}$, avec de vastes réservoirs hydroélectriques et des marais saumâtres côtiers, principalement le long de la côte atlantique.

La plupart des cours d'eau algériens prennent leur source dans l'Atlas tellien et coulent en direction du nord vers la Méditerranée ; c'est le cas notamment du système de l'oued

Figure 15. La sous-région de l'Afrique du Nord.

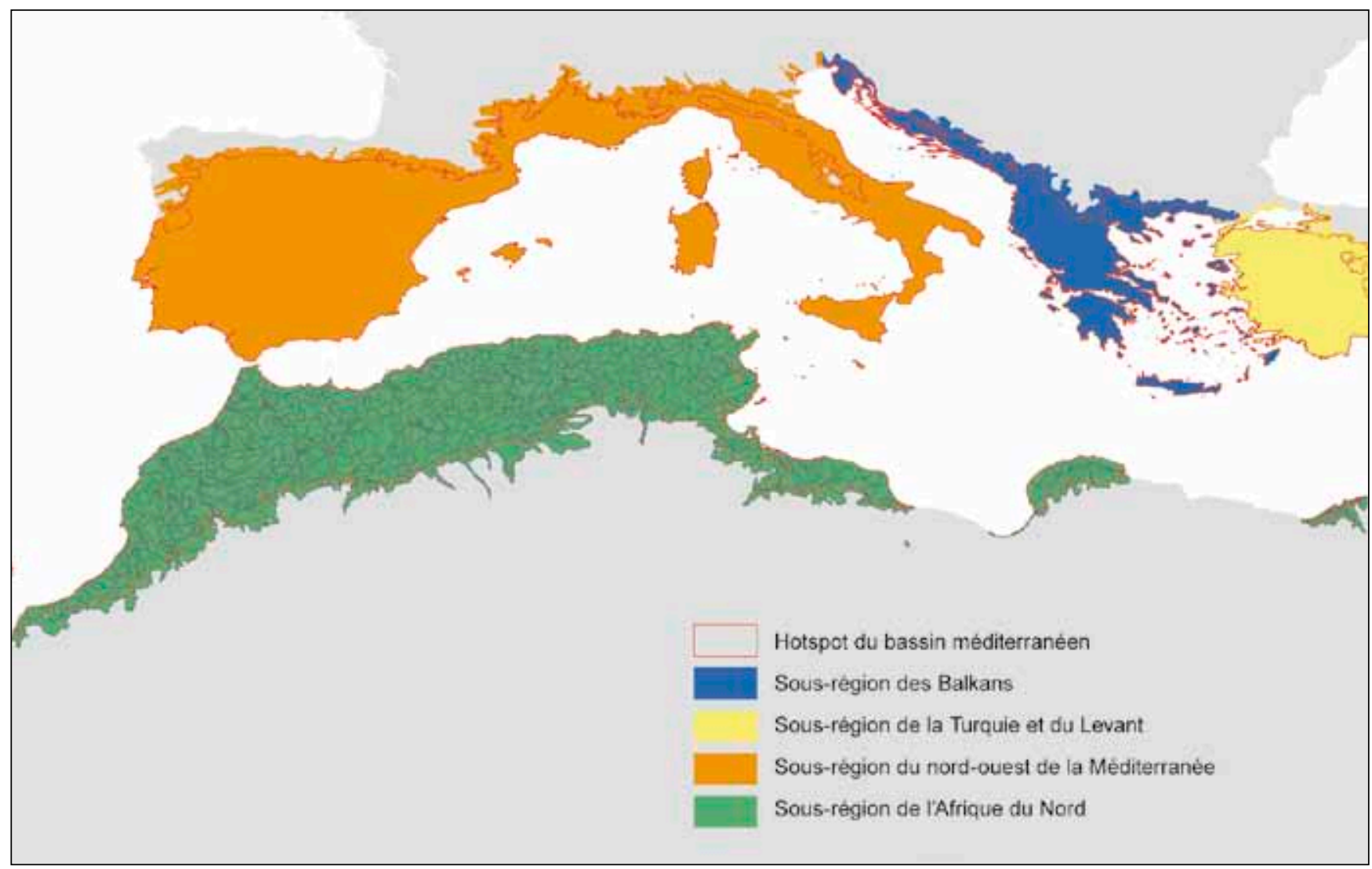


Chellif (longueur d'environ $550 \mathrm{~km}$ ) et du système de l'oued Seybouse (longueur d'environ $180 \mathrm{~km}$ ). L'Algérie possède aussi de nombreuses petites rivières se limitant à la côte montagneuse méditerranéenne de l'Atlas tellien. Des vestiges de grands lacs et de zones marécageuses, peuvent toujours être observés, à savoir le lac de Fetzara (Annaba), la Macta, la Grande Sebkha (près d'Oran) et un ensemble complexe de marais et de petits lacs près d'El Kala (Samraoui et Samraoui 2008). Les grottes de Ghar Boumaaza, découvertes en 1931, font partie du plus grand système hydrologique souterrain d'Afrique.

En Tunisie, le principal et unique cours d'eau pérenne est le système de l'oued Medjerda (longueur de $450 \mathrm{~km}$ ) qui prend sa source en Algérie et finit dans le golfe de Tunis (pour se jeter dans la mer Méditerranée). Les deux plus grands lacs, le lac de Tunis et le lac Ichkeul, sont saumâtres donc quelques espèces d'eau douce sont présentes dans les marais environnants ainsi que dans l'oasis de Nouail, près du Chott El Jérid. Certaines rivières de montagne dans la Kroumirie présentent un intérêt considérable en termes de faune et de flore, et elles sont probablement un hotspot d'endémisme (Korbaa et al. 2014 ; Samraoui et Al Farhan 2015).

En Libye, il n'existe pas de cours d'eau permanents mais une multitude de sources, de seguias (canaux d'irrigation), de mares, de puits artificiels et d'oasis, ainsi que des marais salants. Les principales régions dotées d'oasis sont les régions de Ghat, Sabha et Kufrah. Le Grand fleuve artificiel est un gigantesque système de canalisations qui transportent de l'eau située en profondeur dans la nappe aquifère de grès nubien dans le sud de la Libye, jusqu'aux grandes villes du nord. Les lacs de cratère du Tibesti, comme le Trou au Natron, sont salins ou alcalins.

Le delta du Nil en Égypte est une zone fertile nichée dans l'un des déserts les plus arides au monde. II s'étend sur une longueur d'environ 175 km, de son extrémité sud (au Caire) jusqu'à la mer Méditerranée, et sur une largeur d'environ $260 \mathrm{~km}$ au niveau de la côte. Depuis la construction du haut barrage d'Assouan (achevé en 1970), le débit de l'eau au sein du delta a diminué et cette zone ne fait plus l'objet d'inondations annuelles. Par conséquent, le Nil n'occupe aujourd'hui que deux canaux principaux. Les principales zones humides du delta sont les lacs côtiers de Manzala, Burullus, Idku et Maryut. Le delta du Nil fait partie de l'un des sites les plus importants au monde pour les oiseaux migratoires. La faune d'eau douce est typique de la province Nilo-Soudanaise caractérisée par sa faune ichtyologique. Les établissements humains et les terres agricoles couvrent la quasi-intégralité du delta, à l'exception de quelques zones à l'extrême nord du delta. Les zones humides restantes sont rapidement converties pour l'agriculture et elles ont perdu plus de $50 \%$ de leur superficie initiale au cours du siècle dernier en raison de la mise en valeur des terres à des fins agricoles, de la sédimentation et de l'érosion. Le delta a connu une érosion de près de $2 \mathrm{~km}$ depuis les années 1960, en raison de la perte de dépôts sédimentaires suite à la construction du haut barrage d'Assouan, et il est soumis à une intrusion croissante de l'eau salée. Les polluants s'accumulent dans le delta au fur et à mesure que l'agriculture et les établissements humains augmentent ; les précédentes inondations annuelles qui intervenaient en amont ne sont plus là pour supprimer ces polluants. Les espèces exotiques envahissantes, comme la jacinthe d'eau (Eichhornia crassipes) et l'écrevisse de Louisiane (Procambarus clarkii), menacent la biodiversité autochtone.

\section{Aperçu des ZCB}

Pour la sous-région de l'Afrique du Nord, nous avons étudié 1223 sous-bassins versants (résolution HydroBASINS de niveau 8), couvrant une superficie de $921006 \mathrm{~km}^{2}$. Au sein de ces sous-bassins versants, 1025 ZCB proposées ont été identifiées comme remplissant les critères de ZCB en raison des groupes taxonomiques d'eau douce évalués à cet endroit (Figure 16). Concernant les espèces de déclenchement de ZCB, 109 espèces de ce type ont été identifiées (Tableau 10). Le nombre relativement élevé d'espèces remplissant a priori le Critère 3 s'explique par la présence de nombreuses espèces restreintes à des écorégions d'eau douce individuelles, la région de l'Afrique du Nord dans son ensemble étant délimitée en seulement deux grandes écorégions d'eau douce. Une consultation ultérieure auprès des parties prenantes concernant ces ZCB proposées a conduit à la désignation et à la délimitation de 43 ZCB validées qui incluent les aires de répartition de 92 espèces remplissant au moins l'un des critères de ZCB (Tableau 11, Figure 17 et Annexe IV, Tableau B). Le nom des ZCB et le nombre d'espèces de déclenchement sont indiqués dans le Tableau 12.

Tableau 10. Synthèse des ZCB proposées. Nombre de sous-bassins versants proposés en tant que ZCB en raison de la présence d'espèces menacées $(C 1)$, d'espèces ayant une aire de répartition restreinte (C2), et de communautés restreintes à un biome (C3) pour chaque groupe taxonomique. À noter : les chiffres de la colonne " Tous les critères » représentent le nombre total de bassins versants distincts qualifiés, et ne correspondent donc pas à la somme des lignes.

Nombre de sous-bassins versants qualifiés

\begin{tabular}{|lcccc|}
\hline & Tous les critères & C1 & C2 & C3 \\
\hline Poissons & 815 & 731 & 58 & 357 \\
\hline Mollusques & 476 & 316 & 200 & 247 \\
\hline Odonates & 487 & 482 & 58 & 2 \\
\hline Plantes & 674 & 672 & 218 & 0 \\
\hline Total & $\mathbf{1 , 0 2 5}$ & $\mathbf{9 6 8}$ & $\mathbf{3 5 6}$ & $\mathbf{4 4 9}$ \\
\hline
\end{tabular}

Tableau 11. Synthèse des ZCB validées. Nombre de sous-bassins versants validés en raison de la présence d'espèces menacées (C1), d'espèces ayant une aire de répartition restreinte (C2), et de communautés restreintes à un biome (C3) pour chaque groupe taxonomique. À noter : les chiffres de la colonne "Tous les critères" représentent le nombre total de bassins versants distincts qualifiés, et ne correspondent donc pas à la somme des lignes.

Nombre de sous-bassins versants qualifiés

\begin{tabular}{|lcccc|}
\hline & Tous les critères & C1 & C2 & C3 \\
\hline Poissons & 33 & 32 & 11 & 19 \\
\hline Mollusques & 28 & 24 & 19 & 9 \\
\hline Odonates & 26 & 21 & 18 & 2 \\
\hline Plantes & 36 & 35 & 21 & 0 \\
\hline Amphibiens (complémentaire) & 4 & 4 & 0 & 1 \\
\hline Total & $\mathbf{4 3}$ & $\mathbf{4 2}$ & $\mathbf{4 0}$ & $\mathbf{2 2}$ \\
\hline
\end{tabular}




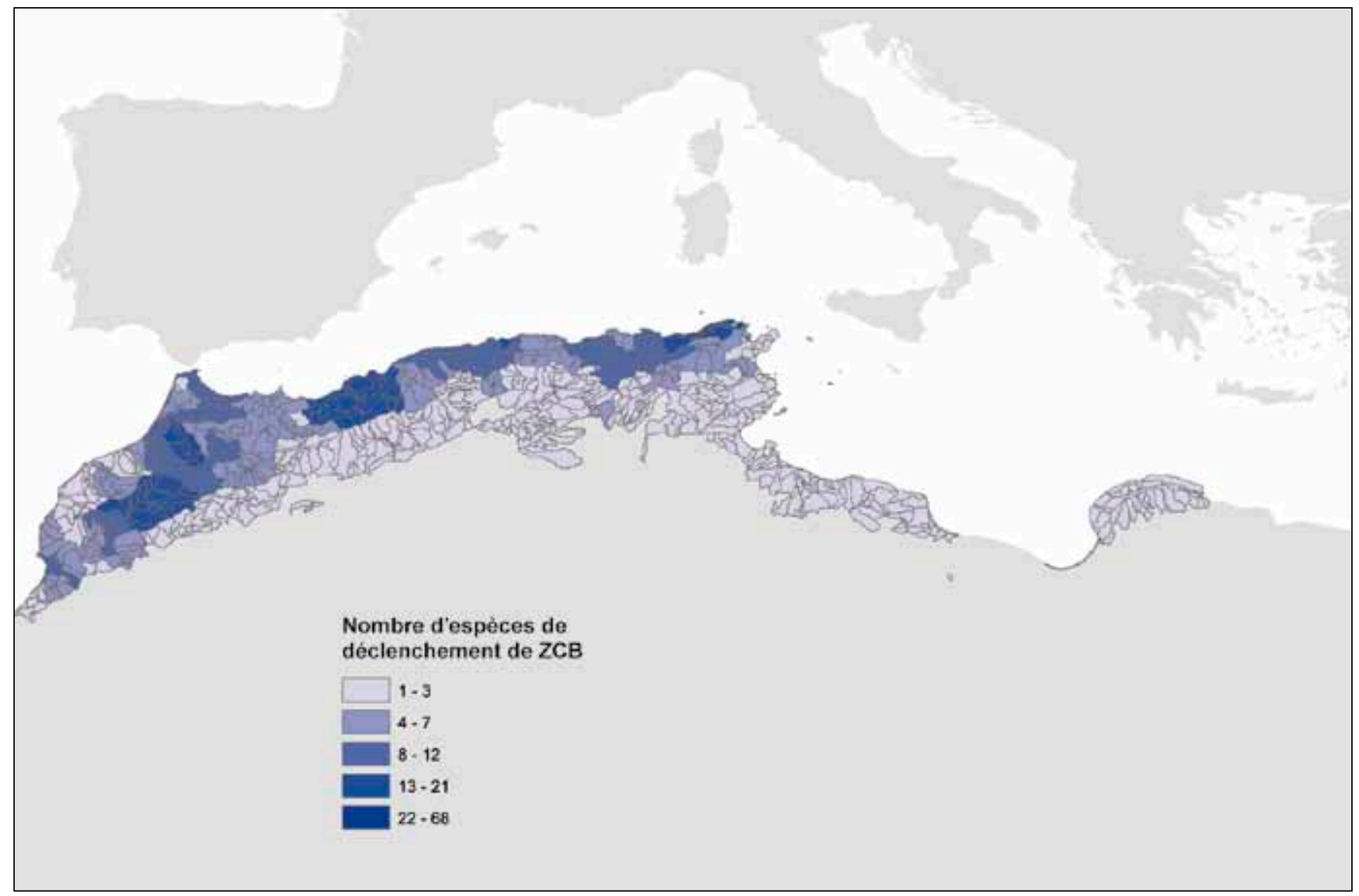

Figure 16. Ensemble des ZCB d'eau douce proposées avec indication du nombre d'espèces de déclenchement de ZCB dans la sous-région de l'Afrique du Nord.

Figure 17. Ensemble des ZCB validées avec indication du nombre d'espèces de déclenchement de ZCB dans la sous-région de l'Afrique du Nord.

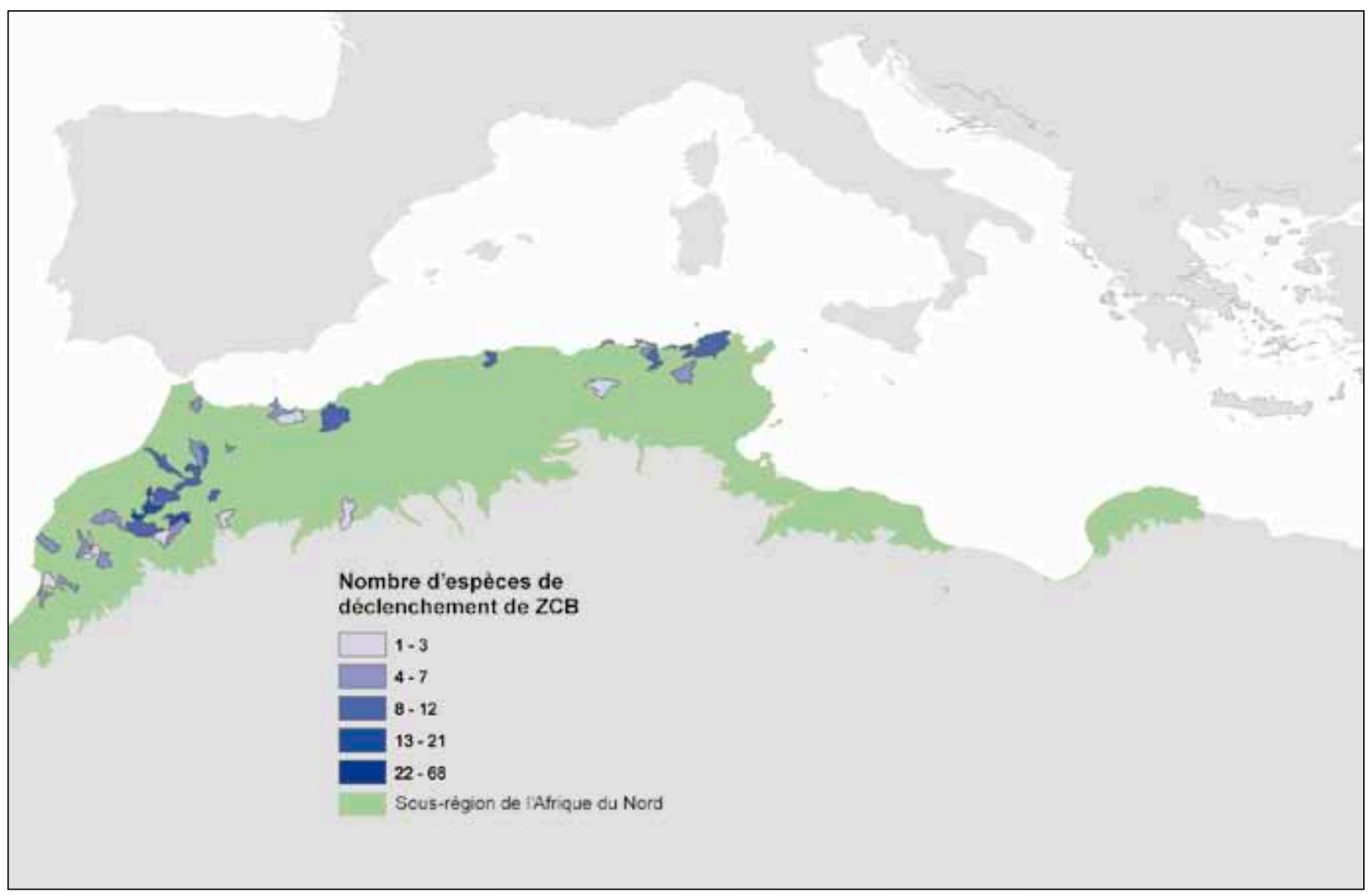


Tableau 12. Liste des ZCB d'eau douce validées pour les bassins versants de la sous-région de l'Afrique du Nord, indiquant le nombre d'espèces de déclenchement de ZCB, les sites AZE (*) et, si elles ont été identifiées, les Zones focales pour chaque ZCB. Les ZCB transfrontalières figurent à la fin du tableau.

\begin{tabular}{|c|c|c|c|}
\hline Pays & Nom de la ZCB ${ }^{1}$ & $\begin{array}{l}\text { Nombre d'espèces } \\
\text { de déclenchement }\end{array}$ & Zones focales \\
\hline Algérie & Beni Belaid & 9 & \\
\hline Algérie & Hauts Plateaux & 3 & $\begin{array}{l}\text { Emplacements de l'espèce endémique Aphanius apodus qui est proche de } \\
\text { l'extinction, entre Aïn Mlila et Batna }\end{array}$ \\
\hline Algérie & Oued el Harrach & 8 & Rivière Melifi - une rivière sur une colline (importante pour Barbus leptopogon) \\
\hline Algérie & Oued Zhour & 4 & \\
\hline Algérie & Seybouse catchment & 11 & $\begin{array}{l}\text { Cours supérieur du Seybouse, important pour Calopteryx exul et Gomphus } \\
\text { lucasii }\end{array}$ \\
\hline Algérie & Tafna Catchment & 10 & \\
\hline Algérie & Western Numidia* & 7 & Lac de Fetzara (ZICO, site Ramsar et important pour Pleurodeles poireti) \\
\hline Maroc & Abid river downstream & 14 & \\
\hline Maroc & Arhreme River* & 5 & Les puits \\
\hline Maroc & Assif El Mal & 5 & Bassin versant supérieur \\
\hline Maroc & Assif El Mal east & 2 & Sources et rivières du bassin versant supérieur \\
\hline Maroc & Assif Meloul river & 13 & Les deux lacs de montagne (oligotrophes) et leur source \\
\hline Maroc & Lower Moulouya & 3 & Sites de frai de l'espèce anadrome Alosa algeriensis \\
\hline Maroc & Lower Souss and tributaries & 1 & \\
\hline Maroc & M'Goun river basin & 1 & \\
\hline Maroc & Middle N'Fiss river & 1 & Sites importants pour les populations de Luciobarbus magniatlantis \\
\hline Maroc & Middle Oum Er Rbia - Beni Mellal & 14 & Importantes populations de Ptercapoeta maroccana et Carasobarbus harterti \\
\hline Maroc & Middle Upper Moulouya & 8 & \\
\hline Maroc & Oued Amizmiz & 4 & Source d'Imintala \\
\hline Maroc & Oued Bouhlou & 6 & \\
\hline Maroc & Oued Bouregreg & 8 & Plaine d'Oulmes et estuaire de Bouregreg \\
\hline Maroc & Oued Imouzzer Kandar & 8 & Dayat Aoua (lac endoréique) et Dayat Hachlaf \\
\hline Maroc & Oued Ksob - Igrounzar & 5 & Zones côtières du cours d'eau \\
\hline Maroc & Oued Lakhdar & 12 & \\
\hline Maroc & Oued Laou & 5 & Partie occidentale de Chefchaouen, importante pour les plantes aquatiques \\
\hline Maroc & Oued Massa & 6 & $\begin{array}{l}\text { Emplacement d'Unio foucauldianus dans le bassin versant supérieur et d'autres } \\
\text { sources, et puits importants pour les escargots }\end{array}$ \\
\hline Maroc & Oued Tizguite and Oued Ouaslane & 5 & Section supérieure de ces deux oueds \\
\hline Maroc & Oued Ziz Errachidia & 3 & \\
\hline Maroc & Saidia Coastal Plain & 6 & \\
\hline Maroc & Sehb El Majnoune & 6 & \\
\hline Maroc & Tifnout basin & 6 & Lac Ifni abritant l'espèce endémique Salmo sp. \\
\hline Maroc & Tigrigra stream & 9 & Source d'Aghbal \\
\hline Maroc & Upper Dades & 4 & La partie la plus haute du bassin versant, au-delà de $2000 \mathrm{~m}$ \\
\hline Maroc & Upper Oued N'Fiss & 2 & Sites importants pour les populations de Luciobarbus magniatlantis \\
\hline Maroc & Upper Oum Er Rbia & 8 & Lac de montagne d'Aguelmame Azigza \\
\hline Maroc & $\begin{array}{l}\text { Upper Oum Er Rbia above Kasba } \\
\text { Tadla }\end{array}$ & 10 & Importantes populations de Ptercapoeta maroccana \\
\hline Tunisie & $\begin{array}{l}\text { Cap Serrat - Cap Blanc - Parc } \\
\text { national de l'Ichkeul }\end{array}$ & 8 & \\
\hline Tunisie & Maden River & 9 & \\
\hline $\begin{array}{l}\text { Algérie, } \\
\text { Maroc }\end{array}$ & Figuig Oasis and Oued Saoura & 2 & $\begin{array}{l}\text { Eaux souterraines de la ville de Figuig, abritant l'espèce endémique Luciobarbus } \\
\text { figuigensis, et gueltas d'Algérie abritant l'espèce Aphanius saourensis }\end{array}$ \\
\hline $\begin{array}{l}\text { Algérie, } \\
\text { Tunisie }\end{array}$ & Eastern Numidia & 12 & \\
\hline $\begin{array}{l}\text { Algérie, } \\
\text { Tunisie }\end{array}$ & $\begin{array}{l}\text { El Kala - Les Tourbières de Dar } \\
\text { Fatma transboundary site }\end{array}$ & 9 & \\
\hline $\begin{array}{l}\text { Maroc, } \\
\text { Espagne } \\
\text { (Melilla) }\end{array}$ & Le Grand Nador & 5 & Lagune de Nador et Cap des Trois Fourches \\
\hline $\begin{array}{l}\text { Tunisie, } \\
\text { Algérie }\end{array}$ & Upper Medjarda River & 6 & \\
\hline
\end{tabular}

1 N.D.T. : Les noms des sites ZCB figurant dans les tableaux sont en anglais. Toutefois, les noms des sites ZCB cités dans le corps de ce document ont été traduits en français en précisant leur nom anglais entre crochets (« [nom anglais : XXX] »), afin de permettre au lecteur de faire le lien avec les noms des sites en anglais figurant dans les tableauX correspondants et dans la base de données en ligne (WBDB, World Biodiversity DataBase). 


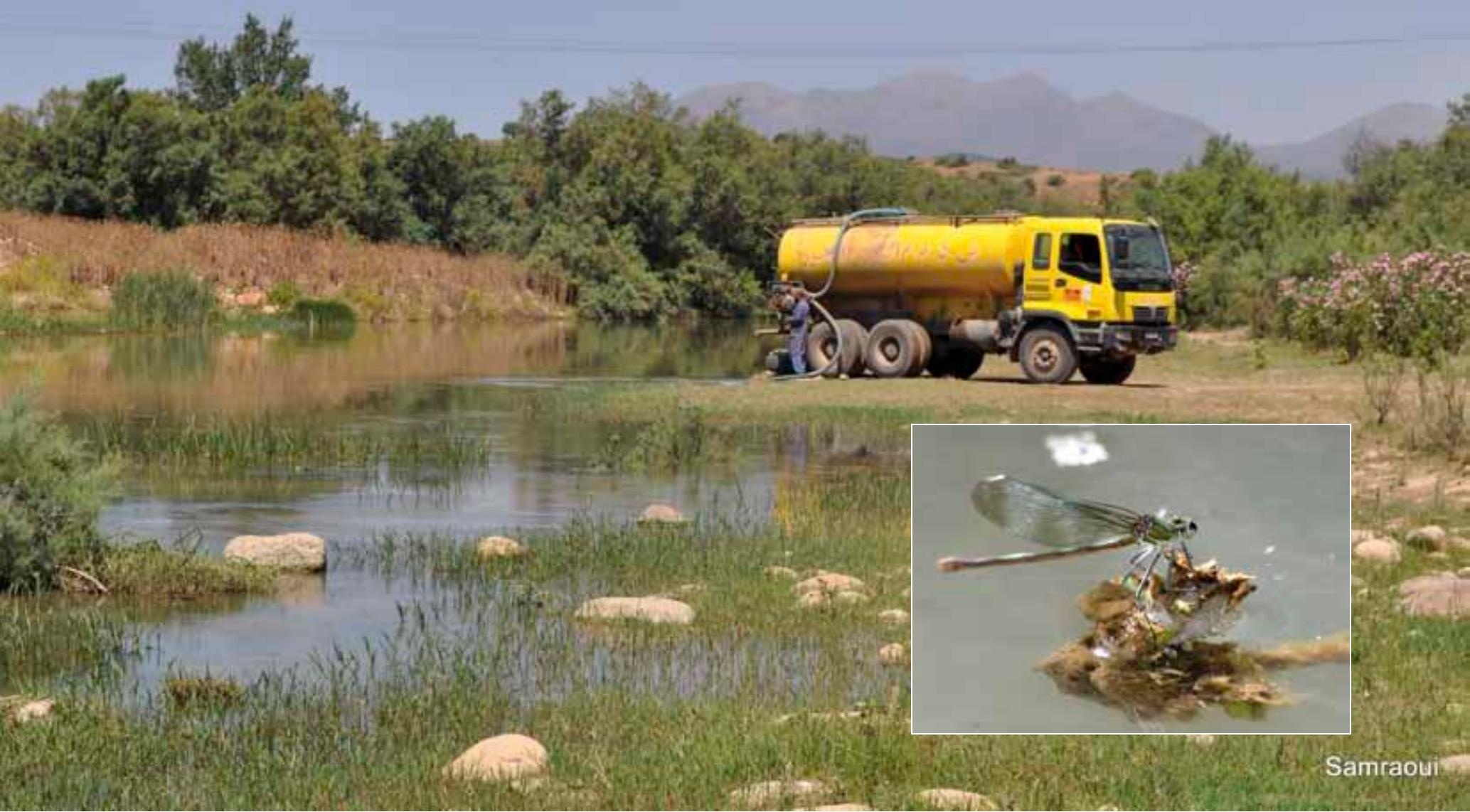

Le captage excessif de l'eau à partir de l'oued de Seybouse (ZCB) [nom anglais : Seybouse catchment], comme l'illustre la photo, a conduit à l'assèchement de nombreux cours d'eau et des habitats présents en leur sein, menaçant leur biodiversité d'eau douce. La demoiselle Calopteryx exul (En danger) (voir encadré) est l'une des huit espèces menacées (composées de poissons, d'odonates, de plantes et de mollusques) vivant dans ce système de cours d'eau. @ Samraoui Boudjéma

\section{Niveaux actuels de protection}

La zone des ZCB d'eau douce validées se trouvant dans les limites géographiques des aires protégées (AP) existantes et des autres ZCB représente $7341 \mathrm{~km}^{2}(12 \%$ de la superficie totale des ZCB d'eau douce validées dans la sous-région de l'Afrique du Nord). Ceci signifie que $88 \%$ de la superficie de ces ZCB d'eau douce se situent à l'extérieur des aires protégées officielles ou d'autres sites reconnus pour leur importance biologique en tant que ZCB (Figure 18). Les ZCB d'eau douce validées ne faisant l'objet d'aucune couverture par une autre $Z C B$ ou $A P$ incluent les ZCB suivantes :

1. Assif El Mal est (Maroc) [nom anglais : Assif El Mal east]

2. Beni Belaid (Algérie)

3. Bassin de l'oued M'Goun (Maroc) [nom anglais: M'Goun river basin]

4. Oued Zhour (Algérie)

5. Cours supérieur du Medjarda (Algérie et Tunisie) [nom anglais : Upper Medjarda River].

\section{Discussion}

La sous-région de l'Afrique du Nord abrite un certain nombre de systèmes de cours d'eau, de lacs et de zones humides importants qui ont été validés en tant que ZCB d'eau douce, dont deux sont aussi des sites AZE (Tableau 12). Toutes les ZCB validées sont situées au Maroc, en Algérie et en Tunisie. Les principales menaces pesant sur les ZCB de cette sousrégion sont les perturbations hydrologiques provoquées par les barrages, les périodes de sécheresse de plus en plus longues, le captage excessif de l'eau, la pollution de l'eau, l'érosion, la salinisation des habitats et les espèces envahissantes. Les difficultés et les recommandations de gestion concernant ces ZCB sont soulignées à nouveau plus bas, dans le cadre de la discussion sur quelques sites sélectionnés.

La ZCB de I'Arhreme [nom anglais : Arhreme River] au Maroc est un bras du Souss et est principalement composée de puits et d'eaux temporaires, avec quelques zones de refuge. Cette ZCB abrite cinq espèces de poissons et de mollusques remplissant les critères de ZCB, dont le mollusque Iglica soussensis (En danger critique) qui est restreint à un unique puits à Tamait Izder, et en raison duquel le site répond aux critères d'admission en tant que site AZE. La pollution et le captage excessif des eaux souterraines représentent des menaces importantes pour les espèces de cette ZCB. L'autre site AZE est la ZCB de la Numidie occidentale [nom anglais : Western Numidia] en Algérie, le seul lieu où le triton de Poiret (Pleurodeles poireti) (En danger) est présent (Samraoui et al. 2012), ainsi que trois espèces de poissons d'eau douce et trois plantes aquatiques remplissant aussi les critères de ZCB. Dans cette ZCB, les principales menaces sont l'expansion urbaine dans les zones montagneuses, l'extraction de sable dans la zone humide de Guerbes (Samraoui et de Bélair 1997 ; Toubal et al. 2014), le captage excessif des eaux souterraines qui a conduit à l'assèchement de nombreuses sources, et l'introduction de la carpe dans les réservoirs des barrages. Un projet d'introduction de carpes (Cyprinus spp.) supplémentaires dans les lacs d'eau douce côtiers a également été discuté. Même si un certain nombre d'aires protégées ont été inscrites en tant que ZCB, cela ne dissipe pas les inquiétudes hydrologiques actuelles. Un projet de gestion des bassins versants est donc recommandé pour traiter la question de l'utilisation de l'eau dans l'ensemble de la 


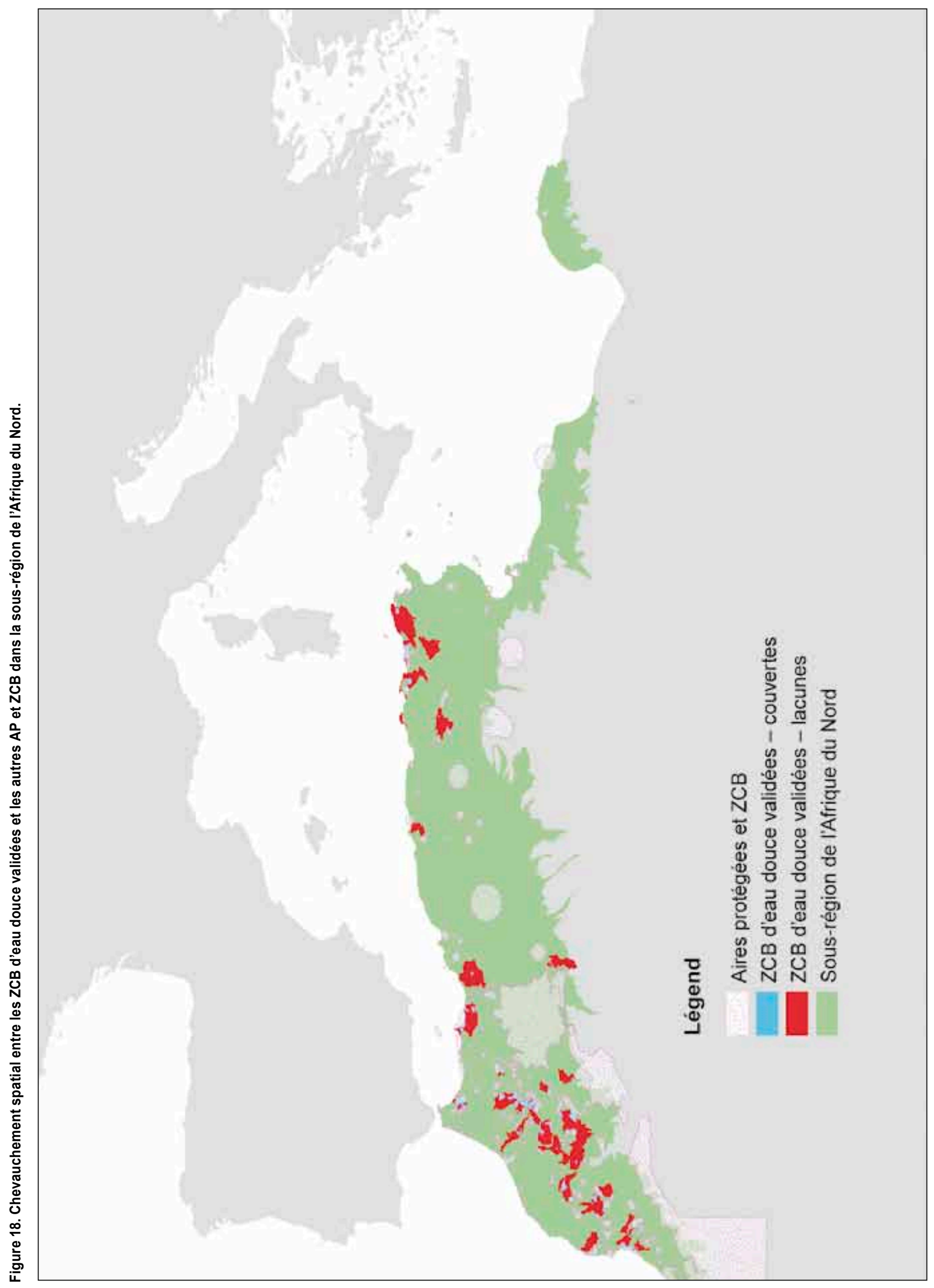


ZCB, laquelle inclut quatre sous-bassins versants liés allant de Skikda (à l'ouest) à Annaba (à l'est), et s'étendant vers l'intérieur pour inclure les zones humides de Guerbes-Senhadja, le lac de Fetzara et le massif de l'Edough. Comme pour de nombreuses ZCB d'eau douce dans lesquelles la menace principale est le captage excessif de l'eau, il est important de désigner des zones de gestion tenant compte de manière adaptée de l'hydrologie sous-jacente du site. Pour cette raison, de nombreuses ZCB d'eau douce incluent les bassins versants environnants associés aux zones focales dans lesquelles sont présentes les espèces de déclenchement de ZCB.

La ZCB Middle Oum Er Rbia - Beni Mellal au Maroc fait partie du grand fleuve permanent en amont du réservoir d'Al Massira. II existe 14 espèces de déclenchement en raison desquelles le site répond aux critères d'admission en tant que $\mathrm{ZCB}$, dont huit mollusques, trois poissons d'eau douce, deux plantes et une libellule. La plupart de ces espèces sont menacées au niveau mondial mais deux espèces de poissons (Carasobarbus harteri et Ptercapoeta maroccana) sont jugées représentatives d'une communauté de poissons dans cette écorégion d'eau douce qui semble proche de l'extinction. Il est même possible qu'une espèce, le grand barbeau (Barbus reinii), se soit éteinte récemment. II convient de noter tout particulièrement que la moule perlière Margaritifera marocana (En danger critique), qui était largement répandue dans le nord-ouest du Maroc, est à présent restreinte à quelques lieux, comme le bassin versant de l'Oum Rbia qui fait partie de cette ZCB. La pollution et le captage excessif de l'eau ainsi que l'envasement sont des problèmes survenant dans l'ensemble de la ZCB, avec de graves répercussions sur les espèces comme $M$. marocana. L'obstruction à la migration des poissons en raison des barrages (il existe six barrages sur l'Oum Er Rbia) représente aussi une menace non seulement pour les espèces de poissons migratoires mais aussi pour M. marocana car elle a besoin de ces poissons-hôtes pour transporter ses larves en amont. Des mesures de protection pour $M$. marocana sont recommandées car il s'agit d'une espèce hautement menacée, de la famille des margaritiféridés dont les représentants sont très peu nombreux au niveau mondial. II est recommandé de déterminer et de mettre en œuvre des flux environnementaux pour ce bassin versant, et de mettre en place des mesures de reboisement dans le bassin versant supérieur.

La ZCB du bassin versant de Tafna [nom anglais : Tafna catchment] en Algérie associe un réseau de zones humides souterraines à des rivières de montagne et à des zones humides côtières, tous reliés hydrologiquement. La ZCB abrite 10 espèces de déclenchement, dont une communauté de mollusques d'eau douce jugés représentatifs de la faune d'eau douce caractéristique de l'écorégion d'eau douce du Maghreb permanent. Comme la plupart des ZCB d'eau douce de cette région, la construction de vastes barrages et le captage excessif de l'eau menacent la biodiversité d'eau douce sur ce site (Samraoui et Samraoui 2008). Un plan de gestion cohérent est nécessaire, en tenant compte de la connectivité du réseau hydrologique et des priorités concurrentielles actuelles des différents ministères concernés.

La ZCB du Cap Serrat - Cap Blanc - Parc national de I'Ichkeul en Tunisie englobe la Garâa Sejenane, une vaste plaine endoréique qui est exceptionnellement riche en zones humides temporaires et comprend une grande diversité d'espèces. Le site abrite Rumex tunetanus (En danger critique), une plante de milieux humides présente uniquement à cet endroit, ainsi que Pilularia minuta (En danger), une plante endémique sténoméditerranéenne. Cette espèce de ptéridophyte amphibie, récemment découverte à Garâa Sejenane, représente la population la plus importante observée dans les mares temporaires méditerranéennes (Daoud-Bouattour et al. 2009 ; Ghrabi-Gammar et al. 2009 ; Daoud-Bouattour et al. 2014). Aujourd'hui, ce site est une mosaïque de terres cultivées et de mares temporaires peu profondes gravement affectées par le drainage, le surpâturage, la pollution et le développement des infrastructures comme la construction de barrages.

Guerbes Chichaya fait partie des zones humides de Guerbes-Senhadja, au sein de la ZCB de la Numidie occidentale [nom anglais : Western Numidia], où les menaces liées aux espèces introduites et au captage excessif de l'eau sont préoccupantes. @ Samraoui Boudjéma

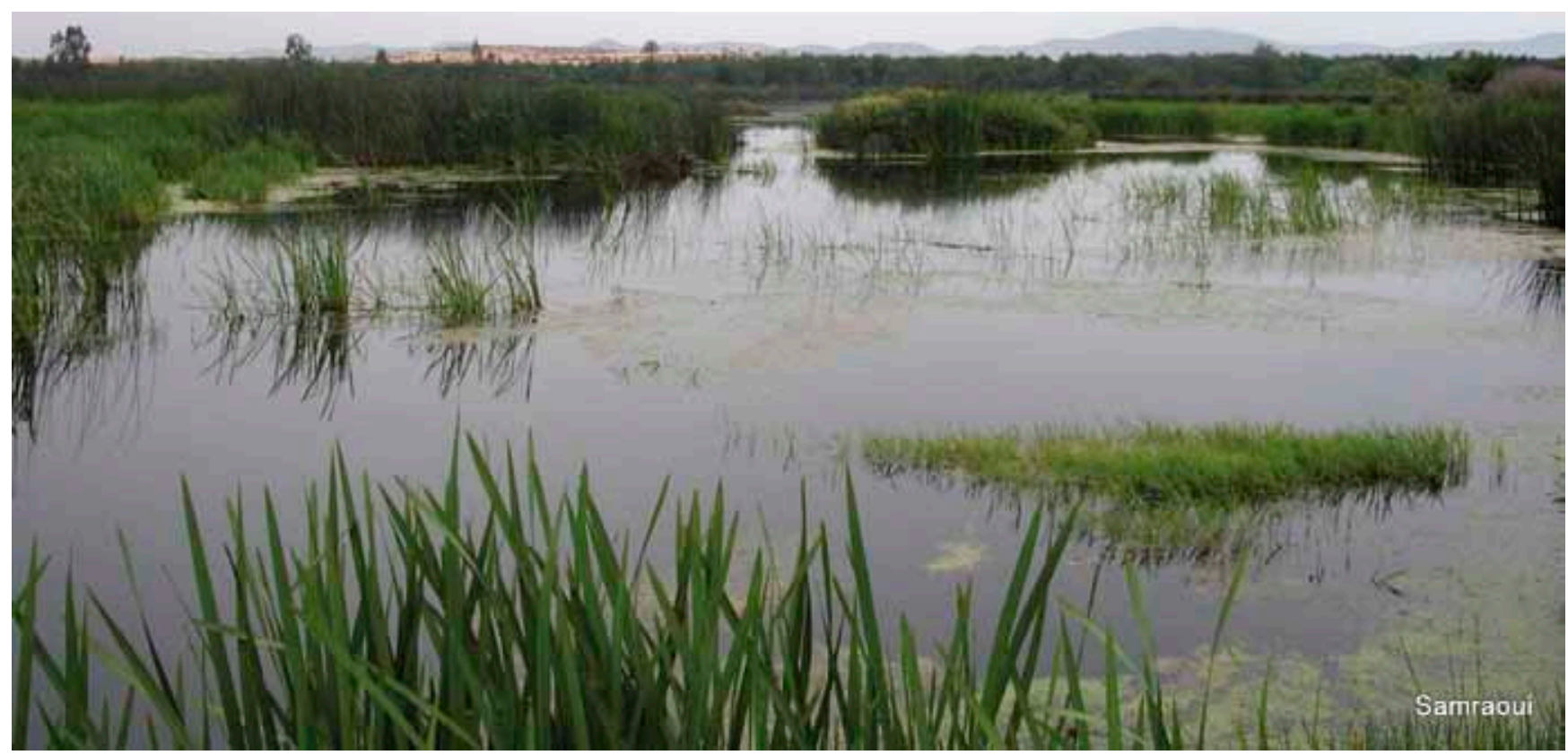




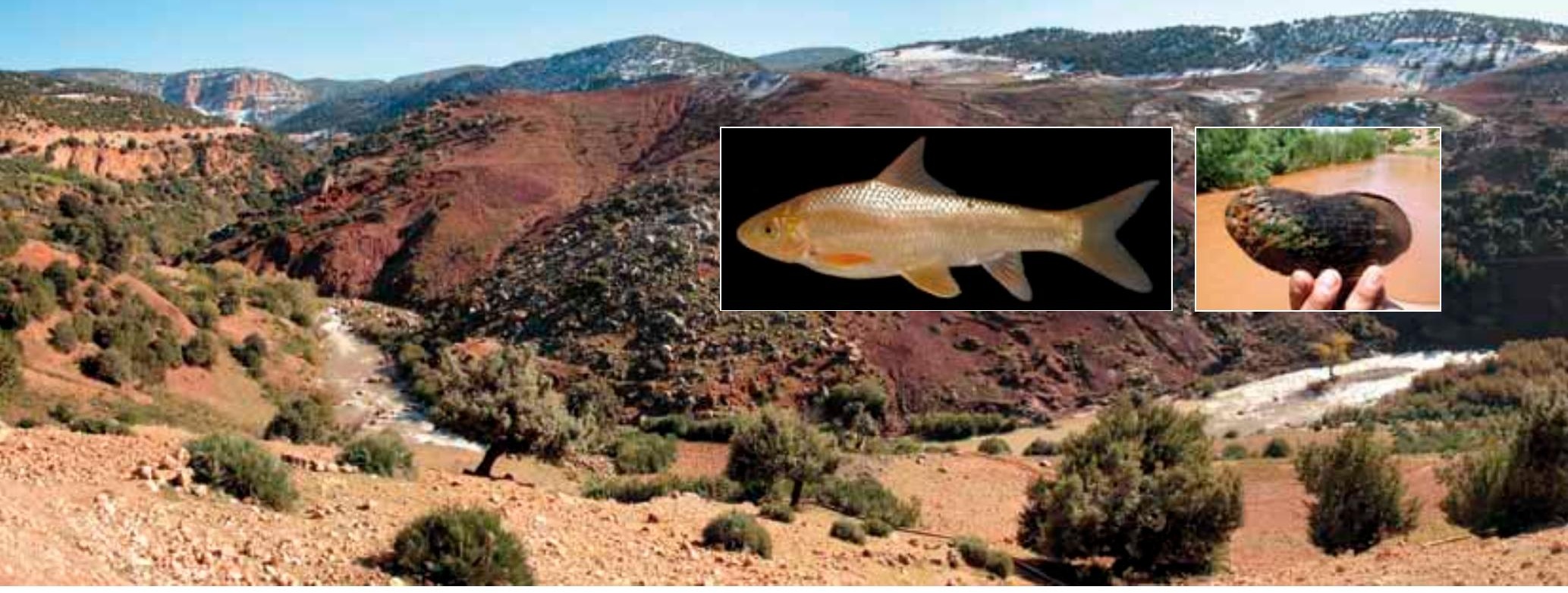

L'Oum Er Rbia, le plus long fleuve marocain, prend sa source dans le Moyen Atlas et se jette dans l'océan Atlantique près d'Agadir. (c) Flikr Mlutlivac42. Ce fleuve est important car il abrite l'espèce de poisson Ptercapoeta maroccana (hautement menacée) et la moule Margaritifera marocana (CR). ๑ Mohamed Ghamizi

Le dernier exemple de site est la ZCB de l'oasis de Figuig et de l'oued Saoura [nom anglais: Figuig Oasis and Oued Saoura], un site transfrontalier s'étendant sur la frontière entre le Maroc et l'Algérie, et incluant des eaux souterraines sous la ville-oasis de Figuig et des mares saisonnières servant de refuge lorsque les oueds sont à sec (gueltas). Le site abrite deux espèces de déclenchement de ZCB, composées de poissons : l'aphanius saharien (Aphanius saourensis) (En danger critique), et Luciobarbus figuiguensis dont l'aire de répartition est restreinte et qui vit principalement à Figuig dans les habitats souterrains. II a récemment été identifié qu'Aphanius saourensis, que l'on croyait autrefois largement répandu, n'était en fait présent qu'au sein de cette seule population subsistant dans le bassin versant de l'oued Saoura ; toutefois, il semble aujourd'hui très probable que cette espèce soit éteinte à l'état sauvage (Freyhof, comm. pers.). La construction d'un barrage sur l'oued Saoura, l'introduction de Gambusia holbrooki qui a conduit à la disparition de poissons similaires et d'autres espèces d'eau douce dans d'autres zones (Samraoui 2002), le captage de l'eau, les sécheresses de plus en plus fréquentes et graves, et le lessivage potentiel de pesticides pulvérisés sur les palmiers ont tous un impact sur les espèces d'eau douce présentes dans cette ZCB. Un programme de reproduction ex situ est en place pour $A$. saourensis ; la restauration de l'habitat, le retrait de Gambusia et un plan de gestion hydrologique de la ZCB sont donc prioritaires pour que cette espèce soit réintroduite dans son milieu naturel.

En conclusion, les principales difficultés sont une fois encore liées à la pénurie, au détournement, à la pollution et à l'utilisation excessive de l'eau. Une plus grande sensibilisation sur la présence de ces espèces endémiques menacées au niveau mondial et les sites critiques pour leur survie est une priorité. Les écosystèmes d'eau douce sont essentiels pour les moyens de subsistance et l'économie des pays d'Afrique du Nord (Juffe-Bignoli et Darwall 2012) mais leur importance reste largement sous-estimée par la population locale et par les décideurs, et ils sont encore considérés comme des zones de friche dont la transformation pour d'autres usages comme l'agriculture est préférable.

La zone humide de Garâa Sejenane, une mosaïque de terres cultivées et de mares temporaires peu profondes, est soumise à un captage de l'eau à des fins d'irrigation des cultures et est également menacée par le surpâturage. Cette zone humide abrite Rumex tunetanus (En danger critique) (voir encadré) et un certain nombre d'autres plantes de milieux humides importantes mais elle ne bénéficie pourtant d'aucune protection. $\odot$ A. Daoud-Bouattour
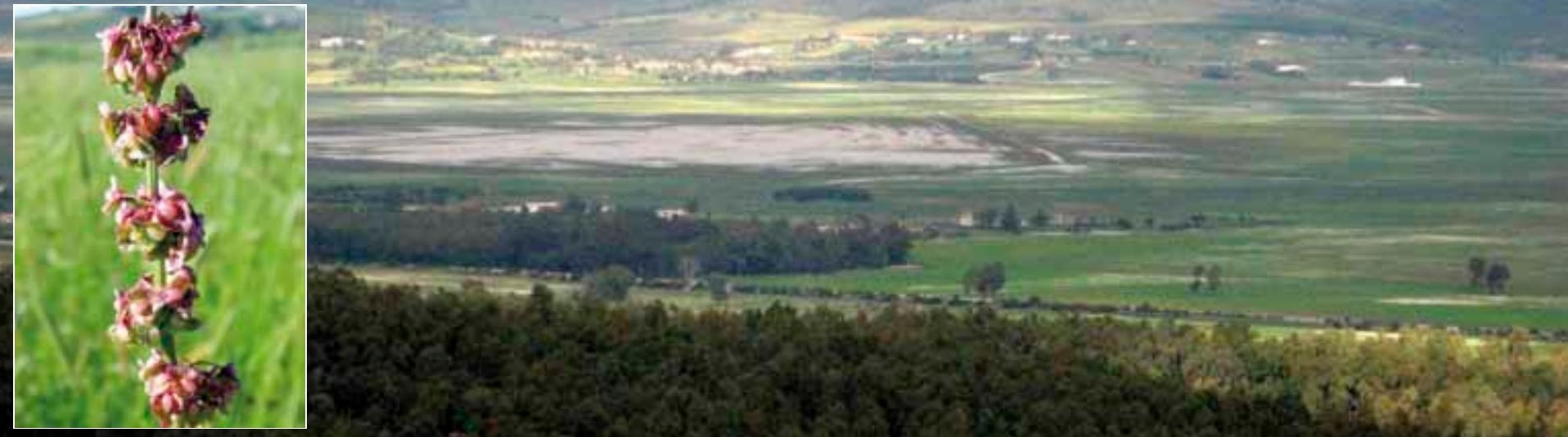


\section{Les ZCB d'eau douce dans la sous-région du nord-ouest de la Méditerranée}

La sous-région du nord-ouest de la Méditerranée (Figure 19) inclut les écorégions d'eau douce suivantes : Zone ibérique méridionale, Zone ibérique occidentale, Zone ibérique orientale, Côte cantabrique - Languedoc, et Péninsule et îles italiennes (Abell et al. 2008). La synthèse suivante repose en grande partie sur les informations obtenues en ligne à partir du site Freshwater Ecoregions of the World (www.feow.org).

La Zone ibérique méridionale comprend deux des plus grands fleuves de la péninsule Ibérique, le Guadiana et le Guadalquivir, qui se jettent dans l'océan Atlantique. Le Guadiana traverse des lacs de montagne (lacs de Ruidera), les zones humides des Tablas de Daimiel et plusieurs grands réservoirs, et son cours supérieur comprend de nombreuses petites cascades. À son embouchure, le Guadiana forme un petit delta et un estuaire comportant des îles, des marais et des bancs de sable. Le Guadalquivir est le plus profond et le seul fleuve navigable de la péninsule Ibérique. II repose sur une dépression bordée par la Sierra Morena au nord et par les cordillères bétiques au sud, et son cours supérieur s'élève au niveau du système prébétique. À son embouchure, il forme un ensemble de marais appelé « Marismas del Guadalquivir » et le site de Doñana, l'une des plus importantes zones humides d'Europe, comprenant des marais et qui est protégée en tant que parc national. Cette écorégion inclut aussi des lacs au sein de steppes salines, des lagunes salines, des zones marécageuses côtières, des réservoirs, de petits lacs, des zones humides et des aquifères.

La Zone ibérique occidentale est constituée de plaines onduleuses, de plateaux, de vallées profondes et de montagnes escarpées. Le Tage et le Douro traversent de vastes bassins cénozoïques, incluant un haut plateau appelé le « Meseta Central ». Le Tage, qui s'étend sur plus de $1000 \mathrm{~km}$, est le plus long fleuve de la péninsule lbérique. Son cours supérieur sillonne le plateau sous forme de gorges profondes et est ponctué de chutes d'eau. II traverse ensuite des collines et des plaines avant de se jeter dans l'océan Atlantique. Le Douro est le troisième plus grand fleuve de la péninsule Ibérique, après le Tage et l'Ėbre. II sillonne les vallées escarpées du système lbérique puis serpente à travers une vallée ouverte pour ensuite aller se jeter dans l'océan Atlantique près de Porto au Portugal. Cette écorégion abrite également une multitude d'estuaires, de marais et de lagunes d'eau douce et saumâtres, tous importants, ainsi que la plus vaste représentation de zones humides constituées de steppes salines en Europe.

La Zone ibérique orientale inclut l'Ėbre, le plus grand fleuve de la péninsule Ibérique. Après avoir traversé les cordillères côtières catalanes, le fleuve se jette dans la mer Méditerranée au niveau du delta de l'Ėbre, qui est une vaste zone humide abritant une biodiversité de grande valeur. Elle comporte un certain nombre de remontées d'eau douce, des lagunes saumâtres et salines, des marais salants, et des zones sablonneuses côtières. Toutefois,

Figure 19. La sous-région du nord-ouest de la Méditerranée.

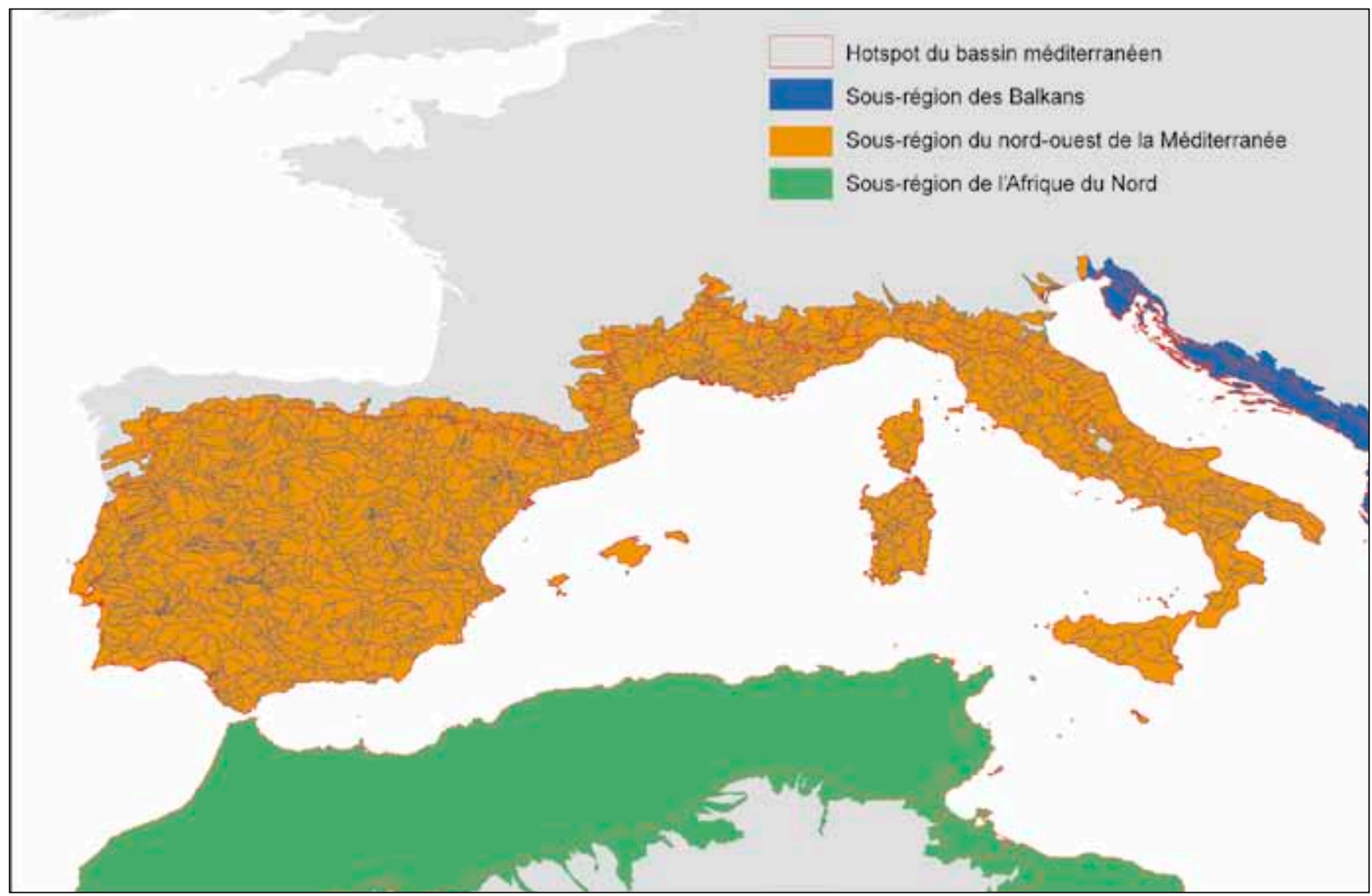


plus de la moitié de la superficie du delta est aujourd'hui dédiée à la culture du riz, entraînant une réduction générale de la salinité et une augmentation de l'eutrophisation, et encourageant également la domination d'espèces d'eau douce envahissantes, en particulier des poissons, des mollusques et des écrevisses. Le Júcar est le deuxième plus long fleuve de l'écorégion ; il prend sa source dans le massif des Montes Universales et se jette dans la mer Méditerranée près de Cullera. Le bassin comprend des zones humides importantes comme l'Albufera de Valencia. Le Ter est le fleuve le plus septentrional au sein de cette écorégion ; il prend sa source dans les Pyrénées et parcourt 209 km avant de se jeter dans la mer Méditerranée. L'un des plus grands lacs espagnols, l'Estany de Banyoles, se situe dans le bassin du Ter.

Le plus grand fleuve de l'écorégion de la Côte cantabrique Languedoc est le cours inférieur du Rhône, de la confluence de l'Isère jusqu'à la mer Méditerranée. À Arles, le fleuve se divise entre le Grand Rhône et le Petit Rhône. Le delta de la Camargue se situe entre ces deux bras : il s'agit d'une zone composée d'un vaste réseau de marais, de dunes, de roselières, d'étangs, de lacs et de lagunes saumâtres à caractère permanent et saisonnier, faisant de ce delta le plus grand d'Europe occidentale. À l'ouest, la Petite Camargue est une autre zone de marais, de lagunes saumâtres et d'étangs.

Concernant la Péninsule et îles italiennes, les fleuves de cette dernière écorégion sont courts en raison de la forme de la péninsule et des Apennins qui coupent la péninsule en deux dans sa longueur et entraînent le ruissellement d'un côté ou de l'autre. Le Tibre est le plus grand fleuve de cette écorégion, et le deuxième plus grand bassin d'Italie. II prend sa source dans l'Apennin septentrional et se jette dans la mer Tyrrhénienne au sud de Rome. Le bassin possède des zones karstiques qui lui sont propres, ainsi que des lacs comme les lacs de Bolsena, de Bracciano et de Vico, qui se sont formés suite à l'effondrement de structures volcaniques.

Bien que la richesse régionale en espèces de poissons d'eau douce soit relativement faible dans cette sous-région d'Europe, elle abrite un nombre important d'espèces menacées dont beaucoup sont localement endémiques, avec une aire de répartition naturelle se limitant à quelques rivières, sources ou cours d'eau. Dans certaines zones, pratiquement chaque cours d'eau possède une faune de poissons d'eau douce qui lui est propre et qui est composée d'espèces endémiques locales. Parmi ces espèces, beaucoup n'ont été que récemment découvertes et décrites, et elles font l'objet d'une protection limitée. Dans de nombreux pays méditerranéens, le captage de l'eau à partir des eaux souterraines ou des rivières et cours d'eau eux-mêmes, n'est soumis en pratique à aucune réglementation et à aucune surveillance, ce qui fait peser des risques sur une grande partie de la biodiversité d'eau douce. En Europe, la région méditerranéenne est la plus riche en mollusques d'eau douce, et en particulier dans la péninsule Ibérique où dominent les petits escargots d'eau douce de la famille des hydrobiidés. Ces espèces sont souvent restreintes à un petit nombre de sources d'eau douce, en particulier dans les régions calcaires de la péninsule Ibérique, de la Méditerranée française et de l'Italie, appartenant à cette sous-région. Concernant la richesse des espèces de plantes aquatiques, l'Espagne, la France et l'Italie figurent dans le Top 5 des pays d'Europe, même si les niveaux d'endémisme
Tableau 13. Synthèse des ZCB proposées. Nombre de bassins versants qualifiés en raison de la présence d'espèces menacées (C1), d'espèces ayant une aire de répartition restreinte (C2), et de communautés restreintes à un biome (C3) pour chaque groupe taxonomique. À noter : les chiffres de la colonne "Tous les critères" représentent le nombre total de bassins versants distincts qualifiés, et ne correspondent donc pas à la somme des lignes.

Nombre de sous-bassins versants qualifiés

\begin{tabular}{|lcccc|}
\hline & Tous les critères & C1 & C2 & C3 \\
\hline Poissons & 1,271 & 1,271 & 170 & 0 \\
\hline Mollusques & 741 & 565 & 523 & 0 \\
\hline Odonates & 336 & 336 & 0 & 0 \\
\hline Plantes & 607 & 606 & 32 & 0 \\
\hline Total & $\mathbf{1 , 3 6 8}$ & $\mathbf{1 , 3 2 5}$ & $\mathbf{6 3 3}$ & $\mathbf{0}$ \\
\hline
\end{tabular}

sont plus faibles dans les zones méditerranéennes. La richesse des plantes aquatiques est particulièrement menacée dans la péninsule Ibérique, surtout en raison d'un ensemble d'espèces endémiques se trouvant dans des habitats vulnérables comme les mares éphémères. La perte de ces habitats sous l'effet du drainage à des fins agricoles représente la plus grande menace.

\section{Aperçu des ZCB}

Au sein de cette sous-région, 1368 ZCB proposées ont été identifiées en tant que sous-bassins versants remplissant les critères de ZCB en raison des groupes taxonomiques d'eau douce évalués (Figure 20, Tableau 13). L'identification se base sur 181 espèces remplissant au moins l'un des critères de ZCB. Un processus de validation de la part des parties prenantes est prévu pour valider et délimiter les ZCB à partir de ce sous-ensemble de sous-bassins versants fluviaux/lacustres de la région.

\section{Discussion}

Des ateliers avec les parties prenantes restent à organiser pour valider le très grand nombre de ZCB d'eau douce proposées et représentatives des sous-bassins versants fluviaux/lacustres remplissant a priori les critères de ZCB. Les pays situés dans cette région du hotspot n'ont pas été éligibles au financement dans le cadre du programme du CEPF, il reste donc nécessaire d'obtenir des fonds auprès d'autres sources afin d'achever ces travaux. Comme pour les autres régions appartenant à ce hotspot, la biodiversité d'eau douce est soumise à une pression considérable, surtout en raison de la mauvaise gestion des ressources en eau (rares et en diminution) alors que celles-ci sont essentielles pour subvenir aux besoins des espèces d'eau douce. De nombreuses espèces endémiques ayant une aire de répartition restreinte, et présentes dans une multitude de petits cours d'eau et de rivières temporaires de la côte méditerranéenne, sont menacées d'extinction au niveau mondial. Malheureusement, sans la mise en place de mesures de sensibilisation, peu de gens déploieront les efforts nécessaires pour conserver ces espèces. 


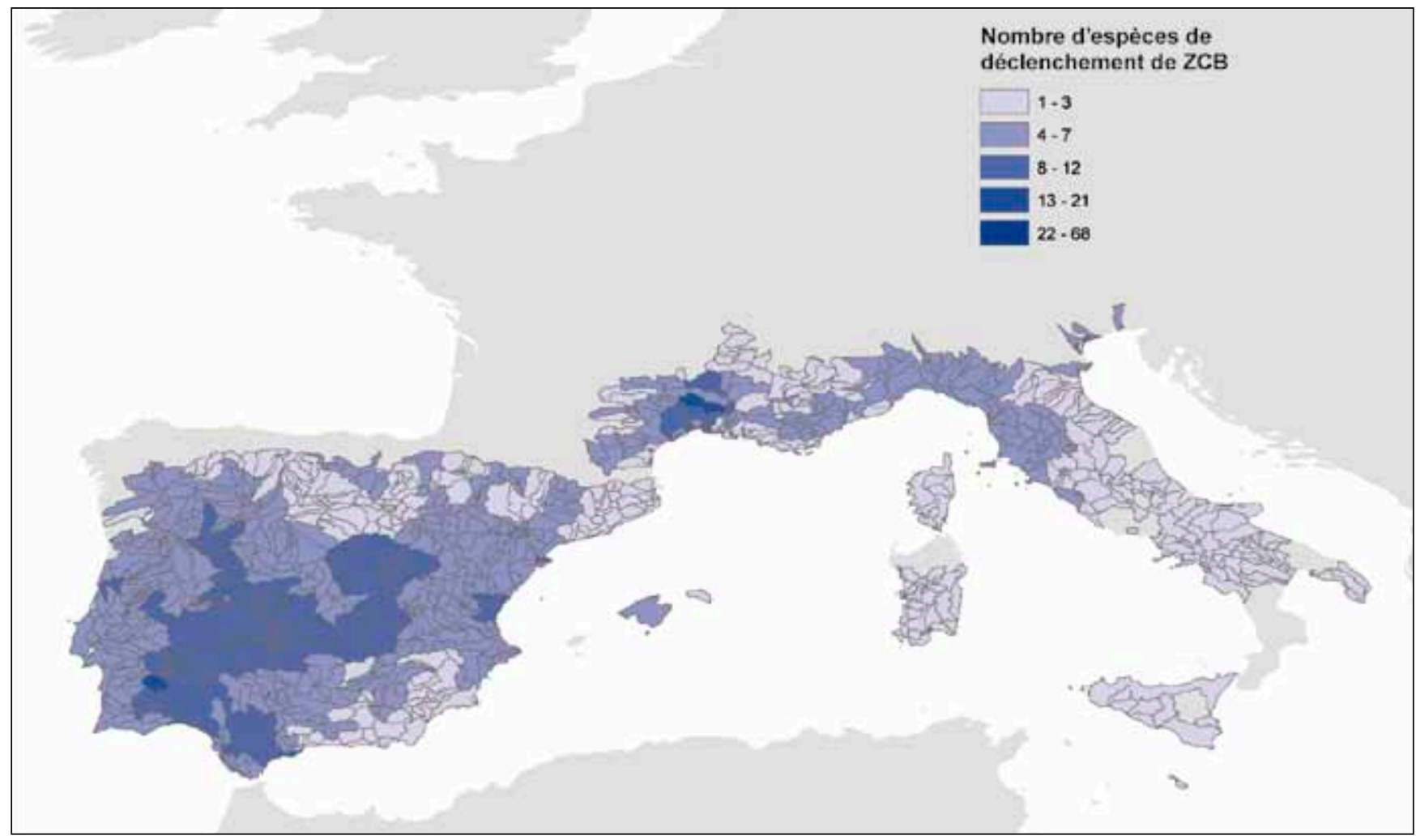

Figure 20. Ensemble des ZCB d'eau douce proposées avec indication du nombre d'espèces de déclenchement de ZCB dans la sous-région du nord-ouest de la Méditerranée. 


\section{Conclusions et recommandations}

Le hotspot du bassin méditerranéen est reconnu depuis longtemps comme un centre de biodiversité d'une importance mondiale selon les critères définissant un hotspot de biodiversité. En effet, d'après ces critères, le site doit abriter au moins 1500 plantes vasculaires endémiques et avoir subi une perte d'au moins $70 \%$ de sa végétation naturelle d'origine (Myers et al. 2000). Toutefois, l'importance de ce hotspot, au niveau de la biodiversité d'eau douce qu'il abrite, était jusqu'à présent mal comprise. Ce projet a permis de confirmer que ce hotspot est d'une importance mondiale pour la biodiversité d'eau douce. Il abrite un nombre exceptionnellement élevé d'espèces d'eau douce endémiques menacées, ce qui a conduit à la validation de nombreux bassins versants fluviaux et lacustres en tant que ZCB d'eau douce et sites AZE. II convient de souligner en particulier le grand nombre d'îles comportant de petites zones humides peu reconnues mais importantes car elles abritent des espèces endémiques menacées généralement omises. Le WWF a récemment estimé qu'il existait plus de 11000 zones humides (naturelles et artificielles) sur 140 îles du bassin méditerranéen (Maragou, comm. pers.).

L'identification des ZCB d'eau douce, telles que présentées ici, a pour vocation de devenir l'une des actions de conservation les plus importantes à mener pour les raisons suivantes. Les ZCB ont déjà été adoptées dans le cadre des objectifs des conventions internationales comme la Convention sur la diversité biologique (en particulier, l'Objectif 11 sur l'expansion du réseau des aires protégées) (UICN et BirdLife International (2013)). Dans le monde entier, les Parties à ces Conventions sont obligées de travailler en vue d'atteindre les objectifs fixés. Les ZCB sont aussi utilisées par de grands donateurs, comme le Fonds de partenariat pour les écosystèmes critiques (CEPF) et la Fondation MacArthur, pour décider de l'attribution géographique des fonds qu'ils versent à des fins de conservation de la biodiversité. Enfin, et il s'agit probablement de la raison la plus importante, les ZCB sont en train d'être intégrées dans les lignes directrices en matière de protection environnementale, appliquées par de nombreux grands donateurs mondiaux, comme le Groupe de la Banque mondiale (IFC), et les sociétés privées. Une fois ces ZCB officiellement reconnues, il appartient aux donateurs et aux acteurs du secteur privé de veiller à ce que des efforts soient déployés pour les protéger et pour éviter, dans la mesure du possible, qu'elles soient affectées négativement. L'identification, la délimitation et la validation des sites en tant que ZCB d'eau douce permettent de situer la biodiversité d'eau douce géographiquement et concrètement.

Concernant la Convention de Ramsar relative aux zones humides, les critères employés pour identifier les ZCB d'eau douce sont aussi équivalents à un grand nombre des critères Ramsar utilisés pour la sélection des sites, de telle manière que les ZCB d'eau douce représentent des sites Ramsar potentiels (Figure 21). Compte tenu des efforts déployés continuellement pour étendre la désignation des sites Ramsar afin d'inclure davantage d'espèces animales n'appartenant pas à l'avifaune (voir Critère 9), les ZCB identifiées ici concernant les poissons, les mollusques, les plantes et les odonates peuvent contribuer favorablement à atteindre cet objectif dans le cadre de l'expansion du réseau de sites au sein du hotspot méditerranéen.

Dans la plupart des cas, le haut niveau de connectivité nécessaire au sein des unités hydrologiques (bassins versants fluviaux et lacustres) passe par une gestion à l'échelle des bassins versants. Les ZCB d'eau douce sont délimitées, comme indiqué plus haut, principalement en utilisant les limites géographiques des bassins versants, afin d'encourager une adoption plus large de la Gestion intégrée des bassins hydrographiques (GIBH) dans l'ensemble du hotspot.

« La Gestion intégrée des bassins hydrographiques (GIBH) est le processus visant à coordonner la conservation, la gestion et le développement de l'eau, des terres et des ressources connexes dans les différents secteurs au sein du bassin d'un cours d'eau donné, afin d'optimiser les bénéfices économiques et sociaux découlant des ressources en eau, ceci de manière équitable, tout en préservant et, si nécessaire, en restaurant les écosystèmes d'eau douce. » [notre traduction] (source : http://wwf.panda.org/ about_our_earth/about_freshwater/rivers/irbm). À moins que l'ensemble des secteurs et des parties intéressées ne travaillent ensemble de manière intégrée au sein d'un bassin versant, les objectifs d'un secteur seront inévitablement en conflit avec ceux d'un autre secteur, conduisant à une situation dans laquelle les objectifs de conservation seront généralement perdants même s'ils présentent un intérêt pour d'autres secteurs.

En tant qu'élément clé de l'approche de GIBH, la mise en œuvre de Flux environnementaux (eFlows) est également essentielle pour la future gestion des ZCB d'eau douce du hotspot méditerranéen. Les eFlows décrivent la qualité, la quantité et le rythme des flux d'eau requis pour alimenter les écosystèmes d'eau douce et subvenir aux besoins des communautés humaines qui dépendent de ces écosystèmes. II est donc recommandé de déterminer et de mettre en œuvre des eFlows pour les ZCB d'eau douce. La première priorité consiste à déterminer les eFlows, selon les besoins, pour tous les sites AZE d'eau douce. L'objectif principal des eflows est de maintenir les fonctions inhérentes aux cours d'eau tout en offrant des bénéfices pour les êtres humains. En pratique, l'équilibre est le plus souvent en faveur des utilisations humaines de ces écosystèmes, en général avec un coût pour la biodiversité. Cette approche déséquilibrée porte non seulement préjudice à la biodiversité d'eau douce mais en fin de compte elle nuit également à la fourniture, de manière durable, des services destinés aux êtres humains.

Dans toute stratégie de gestion de la conservation, il est indispensable d'intégrer une étude de base et de surveiller continuellement la biodiversité aquatique. Les projets visant à promouvoir la systématique en matière de conservation et à cartographier la répartition des espèces sont importants mais malheureusement ils sont rarement perçus en tant qu'impératif scientifique ou de gestion. Dans pratiquement tous les pays méditerranéens, et en particulier dans l'est et le sud du bassin, les travaux de réalisation d'inventaires de base de la biodiversité connaissent du retard. Même les espèces 
Critères de ZCB d'eau douce

\section{Critères Ramsar}

\section{1}

Le site abrite (a priori ou effectivement) un nombre significatif d'individus d'une ou de plusieurs espèces menacées au niveau mondial, ou d'autres espèces d'intérêt pour la conservation.

\section{2}

Le site abrite (a priori ou effectivement) un nombre d'individus non négligeable d'une ou de plusieurs espèces (ou de taxons infraspécifiques) ayant une aire de répartition restreinte.

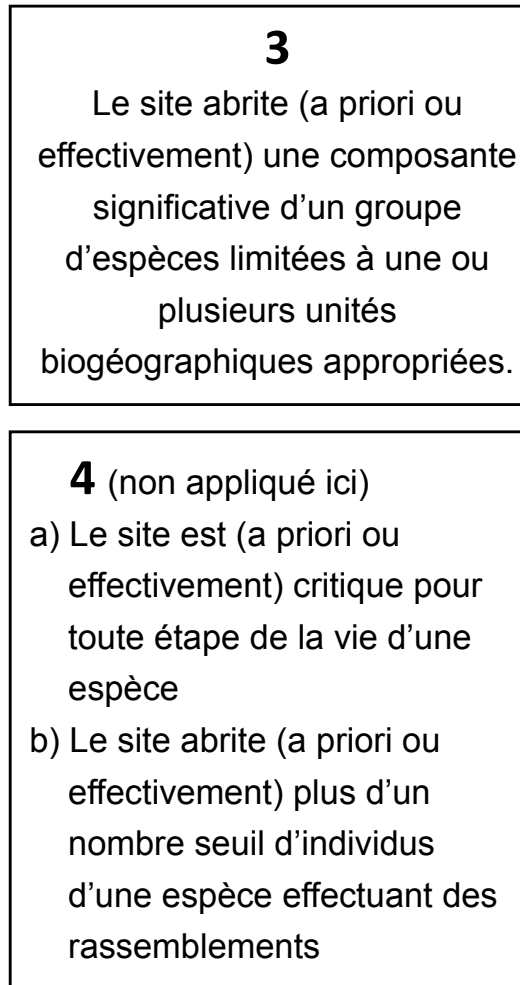

\section{2}

Une zone humide devrait être considérée comme un site d'importance internationale si elle abrite des espèces vulnérables, menacées d'extinction ou gravement menacées d'extinction ou des communautés écologiques menacées.

\section{3}

Une zone humide devrait être considérée comme un site d'importance internationale si elle abrite des populations d'espèces animales et/ou végétales importantes pour le maintien de la diversité biologique d'une région biogéographique particulière.

\section{4}

Une zone humide devrait être considérée comme un site d'importance internationale si elle abrite des espèces végétales et/ou animales à un stade critique de leur cycle de vie ou si elle sert de refuge dans des conditions difficiles.

\section{7}

Une zone humide devrait être considérée comme un site d'importance internationale si elle abrite une proportion importante de sous-espèces, espèces ou familles de poissons indigènes, d'individus à différents stades du cycle de vie, d'interactions interspécifiques et/ou de populations représentatives des avantages et/ou des valeurs des zones humides et contribue ainsi à la diversité biologique mondiale.

\section{8}

Une zone humide devrait être considérée comme un site d'importance internationale si elle sert de source d'alimentation importante pour les poissons, de frayère, de zone d'alevinage et/ou de voie de migration dont dépendent des stocks de poissons se trouvant dans la zone humide ou ailleurs.

\section{9}

Une zone humide devrait être considérée comme un site d'importance internationale si elle abrite régulièrement $1 \%$ des individus d'une population d'une espèce ou sous-espèce animale dépendant des zones humides mais n'appartenant pas à l'avifaune. 
menacées sont souvent mal cartographiées et les informations complémentaires sont difficiles à obtenir. Toutefois, les efforts de conservation ne doivent pas être retardés, même en cas d'incertitudes taxonomiques ou d'insuffisance de connaissances sur la répartition des espèces. En cas d'incertitude, nous devons adopter une approche de gestion adaptative, grâce à laquelle les décisions prises peuvent être modifiées et améliorées en cas de nouvelles informations obtenues suite aux activités de gestion menées. Souvent, les retards au niveau des activités de gestion, dans l'attente de nouvelles informations, conduisent uniquement à la perte ou à la détérioration de la biodiversité que nous cherchons à conserver.

Enfin, il est important que les scientifiques et les ONG spécialisées dans la conservation poursuivent leur engagement en matière d'inventaires, de suivi et d'applications scientifiques pour la conservation de la biodiversité. Les spécialistes doivent aussi informer le public et les autres parties prenantes. La biodiversité d'eau douce est méconnue du grand public et les opérations de communication et d'interprétation, conçues avec soin, jouent un rôle clé pour accroître la sensibilisation aux besoins de la biodiversité. Sans une meilleure compréhension de la part du public des questions relatives à la conservation au sein de nombreuses ZCB, nous n'obtiendrons pas le soutien nécessaire pour gérer ces sites efficacement.

Les prochaines étapes sont les suivantes:

- Publication des ZCB d'eau douce dans la base de données pour la biodiversité mondiale (WBDB, World Biodiversity DataBase). II s'agit de la base de données actuellement gérée par BirdLife International qui assure le stockage, la gestion et la publication de toutes les données sur les ZCB.

- Intégration de l'ensemble des données relatives aux ZCB d'eau douce dans l'outil intégré d'évaluation de la biodiversité (IBAT, Integrated Biodiversity Assessment Tool). L'IBAT est un outil conçu pour faciliter l'accès à une série de couches de données nationales et mondiales, telles que les limites géographiques des aires protégées, des informations biologiques sur les indices de diversité des espèces et des habitats, et les ZCB. II peut être considéré comme un outil polyvalent pour les conservationnistes, les chercheurs et le secteur privé. Concernant le secteur privé, IBAT for Business est déjà utilisé pour intégrer les préoccupations relatives à la diversité au processus décisionnel et à la planification d'entreprise.

- Transmission de ce rapport et des notes stratégiques complémentaires à tous les Ambassadeurs locaux. Nous espérons que les Ambassadeurs locaux (voir Annexe V) seront encouragés à soutenir les actions de conservation dans les ZCB d'eau douce, et à sensibiliser à leur existence et aux besoins en termes de conservation, conduisant à des actions en faveur de la survie à long terme des espèces présentes sur ces sites.

\section{Disponibilité des données relatives aux ZCB}

Toutes les données relatives aux ZCB (y compris les fichiers de formes SIG « Shapefile » relatifs aux ZCB et aux zones focales, ainsi que les fiches techniques relatives aux ZCB individuelles) seront mises à disposition à partir d'un certain nombre de sources en ligne comme :

- L'outil intégré d'évaluation de la biodiversité (IBAT) (https:// www.ibat-alliance.org/ibat-conservation/login) - les données de ZCB seront disponibles lors de la prochaine actualisation de la base de données

- La base de données pour la biodiversité mondiale (WBDB), zone de données sur les ZCB d'eau douce (www.birdlife.org/ datazone/freshwater)

- Le portail de données BioFresh (BioFresh Data Portal), permettant de rechercher des informations sur les ZCB d'eau douce et de soumettre des données complémentaires et des ZCB proposées (http://data.freshwaterbiodiversity.eu/tools)

- L'UICN (Programme mondial sur les espèces, Unité Biodiversité d'Eau douce) (www.iucn.org/species/freshwater). 


\section{Références}

Abell, R., M.L. Thieme, C. Revenga, M. Bryer, M. Kottelat, N. Bogutskaya, B. Coad, N. Mandrak, S.C. Balderas, W. Bussing, M.L.J. Stiassny, P. Skelton, G.R. Allen, P. Unmack, A. Naseka, R. Ng, N. Sindorf, J. Robertson, E. Armijo, J.V. Higgins, T.J. Heibel, and E. Wikramanayake (2008). "Freshwater Ecoregions of the World: A New Map of Biogeographic Units for Freshwater Biodiversity Conservation." BioScience 58(5) 403-414.

Anderson, S. (2002). Identifying Important Plant Areas. Plantlife International.

Annani, F., Al Farhan, A.H. and Samraoui, B. (2012). An ecological survey of aquatic hemiptera of northeastern Algeria (Insecta, Hemiptera). Revue D'ecologie (Terre \& Vie). 67: 423-435.

Bruno D., Belmar O., Sánchez-Fernández D., Guareschi S., Millán, A. and Velasco, J. (2014). Responses of Mediterranean aquatic and riparian communities to human pressures at different spatial scales. Ecological Indicators 45: 456-464.

Carranza, S. and Amat, F. (2005). Taxonomy, biogeography and evolution of Euproctus (Amphibia: Salamandridae), with the resurrection of the genus Calotriton and the description of a new endemic species from the Iberian Peninsula. Zoological Journal of the Linnean Society, 145, 555-582.

Carrizo, S.F., Smith, K.G. and Darwall, W.R.T. (2013). Progress towards a global assessment of the status of freshwater fishes (Pisces) for the IUCN Red List: application to conservation programmes in zoos and aquariums. International Zoo Yearbook, 47, 46-64.

Collen B., Whitton, F., Dyer, E.E., Baillie, J.E., Cumberlidge, N., Darwall, W.R.T., Pollock, C., Richman, N.I., Soulsby, A.-M. and Böhm, M. (2014). Global patterns of freshwater species diversity, threat and endemism. Global Ecology and Biogeography, 23, 40-51.

Cuttelod, A., García, N., Abdul Malak, D., Temple, H. and Katariya, V. (2008). The Mediterranean: a biodiversity hotspot under threat. In: J.C. Vié, C. Hilton-Taylor and S.N. Stuart (eds). The 2008 Review of The IUCN Red List of Threatened Species. IUCN Gland, Switzerland.

Cuttelod, A., Seddon, M. and Neubert, E. (2011). European Red List of Non-marine Molluscs. Luxembourg: Publications Office of the European Union.

Darwall, W.R.T., Holland, R.A., Smith, K.G., Allen, D., Brooks, E.G.E., Katarya, V., Pollock, C.M., Shi, Y., Clausnitzer, V., Cumberlidge, N., Cuttelod, A., Dijkstra, K.-D.B., Diop, M.D., García, N., Seddon, M.B., Skelton, P.H., Snoeks, J., Tweddle, D. and Vié, J.-C. (2011). Implications of bias in conservation research and investment for freshwater species. Conservation Letters, 4: 474-482.

Davidson, N. (2014). How much wetland has the world lost? Long-term and recent trends in global wetland area. Marine and Freshwater Research, 65, 934-941.

Daoud-Bouattour, A., Muller, S.D., Ferchichi-Ben Jamaa, H., GhrabiGammar, Z., Rhazi, L., Gammar, A.M., Karray, M.R., Soulié-Märsche, I., Zouaïdia, H., de Bélair, G., Grillas, P. and Ben Saad-Limam, S. (2009). Recent discovery of the small pillwort (Pilularia minuta Durieu, Marsileaceae) in Tunisia: hope for an endangered emblematic species of Mediterranean temporary pools? C.R. Biol. 332: 886-897.

Daoud-Bouattour, A., Bottollier-Curtet, M., Ferchichi-Ben Jamaa, H., Ghrabi-Gammar, Z., Ben Saad-Limam, S., Rhazi, L. and Muller, S.D. (2014). Effects of hydrology on recruitment of Pilularia minuta Durieu (Marsileaceae), an endangered plant of Mediterranean temporary pools. Aquatic Botany 112: 76- 83.

Juffe-Bignoli, D. and Darwall, W.R.T. (2012). Assessment of the socioeconomic value of freshwater species for the northern Africa region. Gland, Switzerland and Malaga, Spain: IUCN. iv+84 pages.
Juffe-Bignoli, D., Milam, A. and Machsarry, B. (2014). Pre-processing of the WDPA dataset for coverage analyses. UNEP-WCMC Cambridge, UK.

Eken G., L. Bennun, T. M. Brooks, W.R.T. Darwall, L. Fishpool, M. Foster, D. Knox, P. Langhammer, P. Matiku, E. Radford, P. Salaman, W. Sechrest, M. Smith, S. Spector and A. Tordoff (2004). Key Biodiversity Areas as site conservation targets. Bioscience 54 (12): 1110-1118.

Freyhof, J. and Stelbrink, B. (2007). Cobitis illyrica, a new species of loach from Croatia (Teleostei: Cobitidae). Ichthyol. Explor. Freshwaters, Vol. 18, No. 3: $269-275$.

Freyhof, J. (2012). Threatened freshwater fishes and molluscs of the Balkan. Potential impacts of hydropower projects. Unpublished report, ECA Watch Austria \& EuroNatur, $81 \mathrm{pp}$.

Garcia, N., Cuttelod, A. and Abdul Malak, D. (eds.) (2010). The status and distribution of freshwater biodiversity in northern Africa. Gland, Switzerland, UK, and Malaga, Spain: IUCN, 2010. xiii+141pp.

Garcia-Moreno, J., Harrison, I., Dudgeon, D., Clausnitzer, V., Darwall, W., Farrell, T., Savy, C., Tockner, K. and Tockner, K. (2014). Sustaining Freshwater Biodiversity in the Anthropocene. In: The Global Water System in the Anthropocene. Challenges for Science and Governance. Bhaduri A., Bogardi J., Leentvaar J. and Marx S. (Eds.). Springer Cham Heidelberg New York Dordrecht London.

Ghrabi-Gammar Z., Daoud-Bouattour, A., Ferchichi, H., Gammar, A.M., Muller, S.D., Rhazi, L. \& Ben Saad-Limam, S. (2009). Flore vasculaire rare, endémique et menacée des zones humides de Tunisie. Revue d'Ecologie (Terre et Vie), 64: 19-40.

Grimmett, R.F.A. and Jones, T.A. (1989). Important Bird Areas in Europe. Cambridge, UK: International Council for Bird Preservation.

Holland, R.A., Darwall, W.R.T. and Smith, K.G. (2012) Conservation priorities for freshwater biodiversity: the Key Biodiversity Area approach refined and tested for continental Africa. Biological Conservation, 148: 167-179.

IUCN (2013). IUCN Red List of Threatened Species. Version 2013.2 www. iucnredlist.org

IUCN (2014) Key Biodiversity Areas. Task Force Objective 2: To consolidate a standard for the identification of sites contributing significantly to the global persistence of biodiversity. Available at: www.iucn.org/key biodiversity_areas (accessed 13 March 2014).

IUCN and UNEP-WCMC (2014). The World Database on Protected Areas (WDPA), August 2014. Cambridge, UK: UNEP-WCMC.

IUCN and BirdLife International (2013). Key Biodiversity Areas: Identifying areas of particular importance for biodiversity in support of the Aichi Targets. Seventeenth meeting of the Subsidiary Body on Scientific, Technical and Technological Advice, Montreal 14-18 October 2013. CBD.

Jacquemin, G. and Boudot, J-P. (1999). Les libellules (Odonates) du Maroc. Société Française d'Odonatologie, Bois d'Arcy.

Jödicke, R., Arlt, J., Kunz, B., Lopau, W. and Seidenbusch, R. (2000). The Odonata of Tunisia. International Journal of Odonatology 3: 41-71.

Korbaa, M., Ferreras-Romero, M., Bejaoui, M. and Boumaza, M. (2014). Two species of Odonata newly recorded from Tunisia. African Entomology 22: 291-296.

Langhammer, P.F., Bakarr, M.I., Bennun, L.A., Brooks, T.M., Clay, R.P., Darwall, W., De Silva, N., Edgar, G., Eken, G., Fishpool, L., Fonseca, G.A.B. da, Foster, M., Knox, D.H., Matiku, P., Radford, E.A., Rodrigues, A.S.L., Salaman, P., Sechrest, W. and Tordoff, A. (2007). Identification and Gap Analysis of Key Biodiversity Areas as Targets for Comprehensive Protected Area Systems. Gland, Switzerland: IUCN. 
Lehner, B. and Döll, P. (2004). Development and validation of a global database of lakes, reservoirs and wetlands. Journal of Hydrology 296/14: 1-22.

Lehner, B., Verdin, K., Jarvis, A. (2008): New global hydrography derived from spaceborne elevation data. Eos, Transactions, AGU, 89(10): 93-94.

Lehner, B. and Grill, G. (2013). Global river hydrography and network routing: baseline data and new approaches to study the world's large river systems. Hydrological Processes 27: 2171-2186.

Mohtadi, S. 2012. Climate change and the Syrian uprising. Chicago Available at: http://www.thebulletin.org/web-edition/features/climatechange-and-the-syrian-uprising.

Myers, N., Mittermeier, R.A., Mittermeier, C.G., da Fonseca, G.A.B. and Kent, J. (2000). Biodiversity hotspots for conservation priorities. Nature 403, 853-858.

Poff, N.L., Olden, J.D. and Strayer, D. (2012). Climate change and freshwater fauna extinction risk. In: Saving a million species: extinction risk from climate change (ed. by L. Hannah), pp. 309-336. Island Press, Washington.

Ricketts, T.H., Dinerstein, E., Boucher, T., Brooks, T.M., Butchart, S.H.M., Hoffmann, M., Lamoreux, J.F., Morrison, J., Parr, M., Pilgrim, J.D., Rodrigues, A.S.L., Sechrest, W., Wallace, G.E., Berlin, K., Bielby, J., Burgess, N.D., Church, D.R., Cox, N., Knox, D., Loucks, C., Luck, G.W., Master, L.L., Moore, R., Naidoo, R., Ridgely, R., Schatz, G.E., Shire, G., Strand, H., Wettengel, W. and Wikramanayake, E. (2005). Pinpointing and preventing imminent extinctions. Proceedings of the National Academy of Sciences of the United States of America, 102, 18497-501.

Riservato, E. et al. (2009). The Status and Distribution of Dragonflies of the Mediterranean Basin. Gland, Switzerland and Malaga, Spain: IUCN. vii $+33 \mathrm{pp}$.

Samraoui, B., Benyacoub, S., Mecibah, S. and Dumont, H.J. (1993). Afotropical libellulids in the lake district of EI Kala, NE Algeria with a redescription of Urothemis e. edwardsii (Selys) and Acisoma panorpoides ascalaphoides (Rambur) (Anisoptera: Libellulidae). Odonatologica 3 : 365-372.

Samraoui, B. and de Bélair, G. (1997). The Guerbes-Senhadja wetlands: Part I. An overview. Ecologie 28: 233-250.
Samraoui, B. and Menai, R. (1999). A contribution to the study of Algerian Odonata. International Journal of Odonatology 2: 145-165.

Samraoui, B. (2002). Branchiopoda (Ctenopoda and anomopoda) and Copepoda from eastern Numidia, Algeria. Hydrobiologia 470: 173-179.

Samraoui, B. and Samraoui, F. (2008). An ornithological survey of the wetlands of Algeria: Important Bird Areas, Ramsar sites and threatened species. Wildfowl 58: 71-98.

Samraoui, B., Boudot, J-P., Ferreira, S., Riservato, E., Jovic, M., Kalkman, V.J. and Schneider, W. (2010). The status and distribution of dragonflies. In: Garcia, N., Cuttelod, A. and Abdul Malak, D. (Eds) The status and distribution of freshwater biodiversity in Northern Africa. Chapter 5. IUCN, Gland, Switzerland, Cambridge, UK, and Malaga, Spain.

Samraoui, B., Samraoui, F., Benslimane, N., Alfarhan, A. and Al-Rasheid, K.A.S. (2012). A precipitous decline of the Algerian newt Pleurodeles poireti Gervais, 1835 and other changes in the status of amphibians of Numidia, northeast Algeria. Revue d'Écologie (Terre \& Vie) 67: 71-81.

Samraoui, B. and Al Farhan, A.H. (2015). Odonata of mountain streams from Mount Edough, Algeria and Kroumiria, Tunisia. African Entomology: in press.

Smith, K.G., Barrios, V., Darwall, W.R.T. and Numa, C. (Compilers) (in prep.). Status and distribution of freshwater biodiversity in the eastern Mediterranean. IUCN, Cambridge, UK, Malaga, Spain and Gland, Switzerland: ${ }^{*}+{ }^{* *} \mathrm{pp}$.

Strayer, D.L. and Dudgeon, D. (2010) Freshwater biodiversity conservation: recent progress and future challenges. Journal of The North American Benthological Society, 29, 344-358.

Thieme, M.L., Abell, R., Stiassny M.L., Skelton, P. et al. (2005). Freshwater Ecoregions of Africa and Madagascar: A Conservation Assessment. Island Press, Washington DC.

Toubal, O., Boussehaba, A., Toubal, A. and Samraoui, B. (2014). Biodiversité méditerranéenne et changements globaux: cas du complexe de zones humides de Guerbes-Senhadja, Algérie. Physio-Geo: in press.

Zogaris, S., Maclaine, J., Koutsikos, N. and Chatzinikolaou, Y. (2014). Does the river blenny Salaria fluviatilis (Asso, 1801) (Actinopterygii: Perciformes) still survive on the Mediterranean island of Cyprus? Journal of Natural History, DOI: 10.1080/00222933.2013.836761 


\section{Annexe I. Exemples de fiches techniques relatives aux ZCB}

Figure 1. Exemple de fiche technique concernant une ZCB d'eau douce proposée (non validée). Vul = C1, Irr $1=\mathrm{C2}$, Irr $5=$ communauté restreinte à une écorégion.

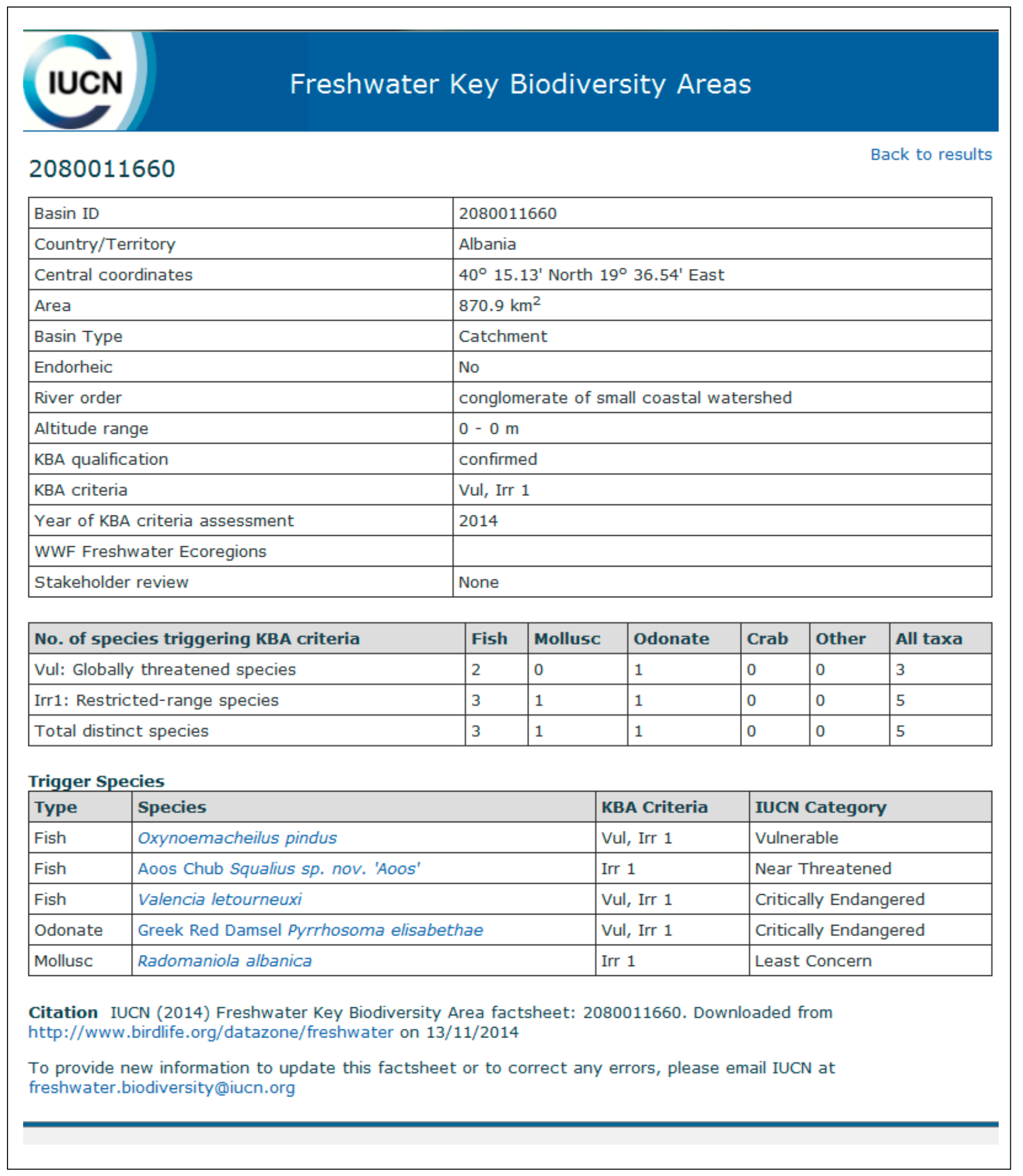


Figure 2. Exemple de fiche technique concernant une ZCB d'eau douce validée, comprenant une description du site et des informations sur les habitats, les pressions et les actions de conservation au sein de la ZCB. Vul $=\mathbf{C 1}, \operatorname{Irr} 1=\mathrm{C2}, \operatorname{Irr} 5=$ communauté restreinte à une écorégion.

\begin{tabular}{|c|c|c|}
\hline \multicolumn{2}{|c|}{ Freshwater Key Biodiversity Areas } & \multirow{2}{*}{ Back to results } \\
\hline Basin ID & 1080045200 & \\
\hline Country/Territory & Morocco & \\
\hline Central coordinates & $32^{\circ} 1.67^{\circ}$ North $7044.83^{\prime}$ West & \\
\hline Area & $2646.8 \mathrm{~km}^{2}$ & \\
\hline Basin Type & Catchment & \\
\hline Endorheic & Yes & \\
\hline Pover order & tributaries that flow into a 1st order river & \\
\hline Avtude range & $0.0 \mathrm{~m}$ & \\
\hline KDA qualification & confirmed & \\
\hline KBA criteria & Vul, Irr 1, 5 & \\
\hline Year of KBA criteria assessment & 2014 & \\
\hline \multicolumn{3}{|l|}{ WWF Freshwater Ecoregions } \\
\hline Stakeholder review & Complete & \\
\hline
\end{tabular}

Site description Endorheic and no permanent rivers. Salty marshland. Important area for FW crustaceans with special life cycles (check shrimp assessment) and birds.

Management required at catchment scale.

Focal area

No

\begin{tabular}{|l|l|l|}
\hline Habitat & Habitat detail & Percentage/cover \\
\hline Wetlands (inland) & $\begin{array}{l}\text { Seasonal / Intermittent /Irregular Rivers, Streams; Seasonal / Intermittent } \\
\text { Freshwater Lakes [> 8 ha]; Seasonl/Intrmttnt Salin/Bracksh/Alkalin } \\
\text { Marsh/Pool }\end{array}$ & - \\
\hline
\end{tabular}

Key biodiversity This catchment was assessed based on fishes, molluscs, plants and odonates.

\begin{tabular}{|l|l|l|l|l|l|l|}
\hline No. of species triggering KBA criteria & Fish & Mollusc & Odonate & Crab & other & All taxa \\
\hline Vul: Globally threatened species & 0 & 4 & 0 & 0 & 2 & 6 \\
\hline Irr1: Restricted-range species & 0 & 4 & 0 & 0 & 1 & 5 \\
\hline Irr5: $25 \%$ of assemblage ecoregion-restricted & 0 & 4 & 0 & 0 & 0 & 4 \\
\hline Total distinct species & 0 & 4 & 0 & 0 & 2 & 6 \\
\hline
\end{tabular}

Trigger Species
\begin{tabular}{|l|l|l|l|}
\hline Type & Species & KBA Criteria & IuCN Category \\
\hline Mollusc & Giustia bodoni & Vul, Irr 1, 5 & Endangered \\
\hline Mollusc & Giustia costata & Vul, Irr 1,5 & Critically Endangered \\
\hline Mollusc & Giustia mellalensis & Vul, Irr 1, 5 & Critically Endangered \\
\hline Mollusc & Giustia saidai & Vul, Irr 1, 5 & Critically Endangered \\
\hline Plant & Girsium ducellien & Vul & Vulnerable \\
\hline Plant & Eryngium varifolium & Vul, Irr 1 & Vulnerable \\
\hline
\end{tabular}

Pressures/threats to key biodiversity

1.1 Housing a urban areas

- urbanisation

2.3 Livestock farming \& ranching

- overgrazing

7.2 Dams \& water management/use

- ground water abstraction for agriculture

11,2 Droughts

\begin{tabular}{|l|l|l|l|}
\hline Protected Amea & Designation & Relationship with KBA & Overlap with KHA (ha) \\
\hline Sahb Al Majnoun & Site of Enological and Ecologcal tnterest (SIBE) & unknown & 0
\end{tabular}

\section{Conservation responses/actions for key biodiversity}

2.1 site/area management

- water managentent to reduce migation mpacts and to allow flooding to attract birds

1 other

regular monitoring of water birds (current) and eco-touram (recomenended)

Acknowledgements

participants of the IUCAN Freshwater KBA validation workshop, Morocco, September 2013.

Potential KBA Champions/Stakeholders

Agency Basin Terisift, Comimune local, GREPOM / BirdLile Morocco

References Uriversity of Marrakech theses and associated publications

Citation tuCN (2014) Freschwater Key piodiversity Area factshent Sehb FI Majnoune. Downloaded from

hitte://www.birdlife, org/datazone/frushwater on 13/11/2014

To provide new informabon to update this factsheet or to correct any errors, please emat wUCN at

freshwater.bindiversityciucn.org 


\section{Annexe II. Participants aux ateliers}
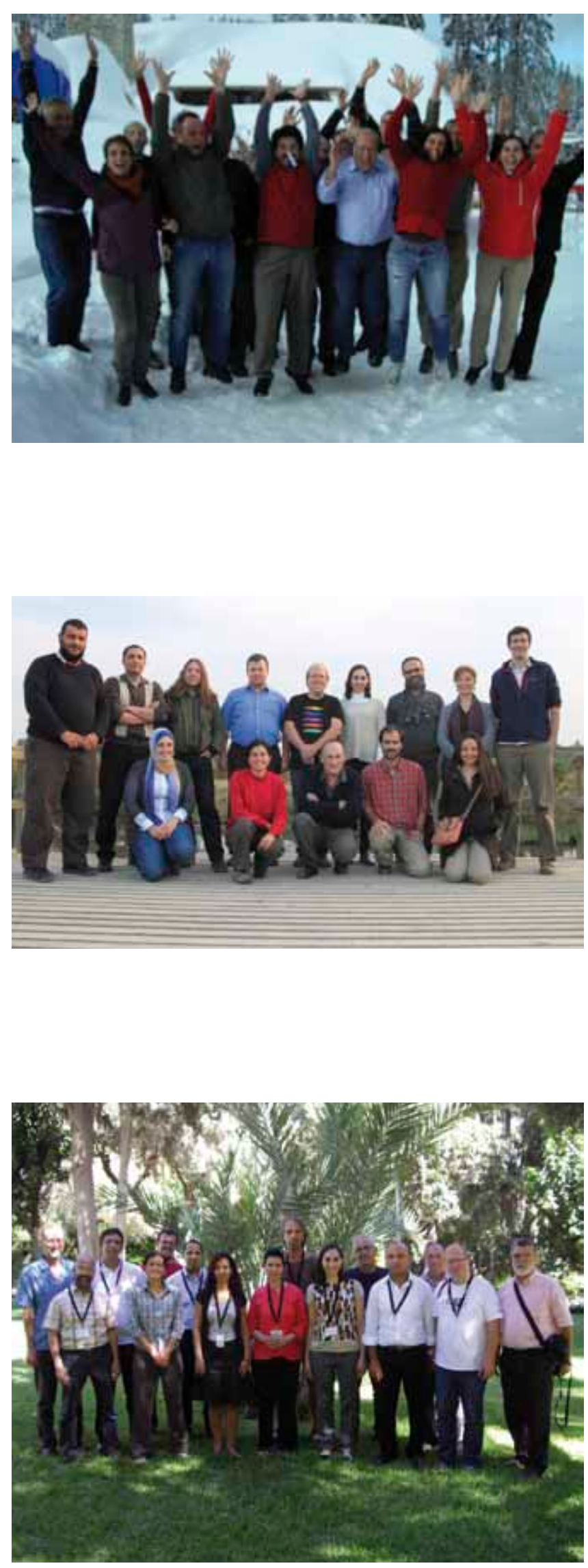

Délimitation et validation des ZCB - Balkans

Aljoša Duplić (State Institute for Nature Protection)

Dejan Kulijer (Musée national de Bosnie-Herzégovine)

Drazen Kotrosan (Musée national de Bosnie-Herzégovine)

Geert De Knijf (INBO, Institut pour la recherche sur la nature et les forêts) loannis Bazos (Université d'Athènes)

Jörg Freyhof (iDiv)

Kanella Radea* (Université d'Athènes)

Krešimir Žganec (Université de Zagreb)

Marko Ćaleta (Université de Zagreb)

Panagiota Maragou (WWF Grèce)

Stamatis Zogaris (Hellenic Centre for Marine Research)

Ulrich Eichelman (EuroNatur / River Watch Austria)

Zoran Marčić (Université de Zagreb)

Zoran Mateljak (Programme méditerranéen du WWF)

William Darwall (UICN)

Savrina Carrizo (UICN)

Violeta Barrios (UICN)

* Participation à distance à la réunion

\section{Délimitation et validation des ZCB - Turquie et Levant}

Jörg Freyhof (iDiv)

F.Güler Ekmekçi (Université de Hacettepe)

Nashat Hamidan (Royal Society for the Conservation of Nature)

Ümit Kebapçi (Université Mehmet Akif Ersoy)

Manuel Lopes Lima (CIIMAR - Université de Porto)

Dirk Van Damme (Université de Gand)

Zuhair Amr (Université jordanienne des sciences et de la technologie) Süreyya İsfendiyaroğlu (Doğa Derneği)

Haifaa Abdulhalim (UICN ROWA, Bureau régional Asie de l'Ouest)

Cagri Gocek (WWF Turquie)

Laith El Moghrabi (Consultant, Jordanie)

Kevin Smith (UICN)

Savrina Carrizo (UICN)

Violeta Barrios (UICN)

\section{Délimitation et validation des ZCB - Afrique du Nord}

Imtinen Ben Hadj Jilani (Institut National Agronomique de Tunisie) Miguel Clavero (Station biologique de Doñana)

Mohamed Dakki (Institut Scientifique de l'Université Mohammed V) Geert De Knijf (INBO, Institut pour la recherche sur la nature et les forêts) Jörg Freyhof (iDiv)

Mohamed Ghamizi (Muséum d'histoire naturelle de Marrakech)

Mohammed Melhaoui (Université Mohammed Premier - Oujda)

Mohamed Noaman (Haut Commissariat aux Eaux et Forêts et à la Lutte Contre la Désertification)

Laila Rhazi (Université Hassan II de Casablanca)

Boudjéma Samraoui (Université de Guelma)

Jamel Tahri (Direction Générale des Forêts)

Dirk Van Damme (Université de Gand)

René Beaumont (Observateur) (Continental Trout Conservation Fund)

William Darwall (UICN)

Savrina Carrizo (UICN)

Violeta Barrios (UICN) 


\section{Annexe III. Zone orientale élargie de la Méditerranée}

Cette annexe présente nos analyses concernant les ZCB d'eau douce dans la zone orientale de la Méditerranée s'étendant au-delà des limites géographiques du hotspot du bassin méditerranéen soutenu par le CEPF.

Les ZCB d'eau douce validées se situent principalement dans trois régions : l'Anatolie centrale turque, le bassin versant du lac de Van en Anatolie orientale, et les bassins versants des cours inférieurs et moyens du Tigre et de l'Euphrate en Syrie et en Irak (et Iran) (Figure 1).

Dans la partie de l'Anatolie centrale située à l'extérieur des limites du hotspot du bassin méditerranéen, il existe quatre ZCB. La ZCB du bassin de Tuz Golu inclut le deuxième plus grand lac turc (lac Tuz) qui est extrêmement salin et s'assèche quasi intégralement en été. II est alimenté par des sources, des cours d'eau et des eaux souterraines (BirdLife International 2014). Cette ZCB abrite une espèce de poisson endémique, Gobio insuyanus (En danger critique), dont la présence n'a été observée que dans la rivière et la source d'Insuyu (Cihanbeyli) (Zone focale au sein de cette ZCB), et qui est menacé par le captage excessif de l'eau, la pollution et la régulation du débit de l'eau. En raison de cette espèce, le site répond aux critères d'admission en tant que site AZE. La ZCB de la rivière Melendiz est un petit cours d'eau situé dans un canyon, qui a subi les effets du captage de l'eau et d'un barrage conduisant à l'assèchement de son cours inférieur en été (Freyhof 2014a). La ZCB répond aux critères d'admission en tant que site $A Z E$ en raison de deux espèces de poissons endémiques En danger critique, Squalius cappadocicus et Gobio gymnostethus, dont la présence n'est plus observée aujourd'hui qu'en amont du barrage. La ZCB du cours supérieur du Zamanti est un cours d'eau d'amont dans la section supérieure de la rivière Seyhan et elle répond aux critères d'admission en tant que site AZE en raison de deux espèces de poissons, la loche Oxynoemacheilus seyhanensis (En danger critique) vivant dans la section principale du cours d'eau, et la truite Salmo platycephalus (En danger) dont la présence est observée dans les affluents de montagne. La truite Salmo platycephalus est menacée par la pêche illégale et par la truite arc-en-ciel (Oncorhynchus mykiss) qui a été introduite, tandis que la loche Oxynoemacheilus seyhanensis subit les effets de la pollution locale et du captage de l'eau, et elle est probablement aussi menacée par l'introduction d'espèces de poissons (Crivelli 2006 ; Freyhof 2014b).

En Anatolie orientale, la seule ZCB validée à l'extérieur du hotspot (et ne faisant pas partie du système du Tigre et de l'Euphrate) est la ZCB du bassin versant du lac de Van. Cinq

Figure 1. Ensemble des ZCB validées avec indication du nombre d'espèces de déclenchement de ZCB dans la zone orientale élargie de la Méditerranée.

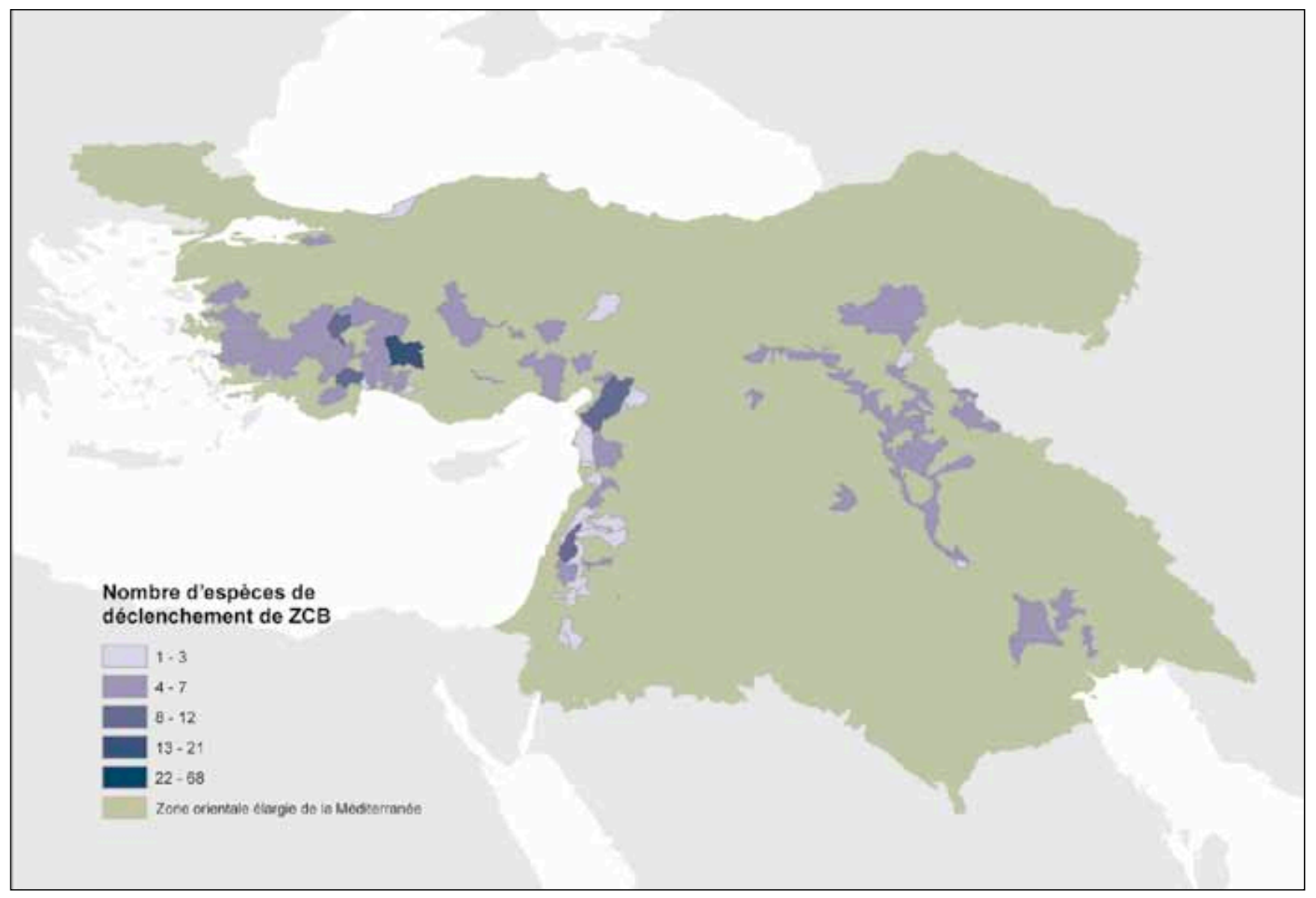


Les sources d'Insuyu sont l'une des Zones focales de la ZCB du bassin de Tuz Golu. La présence de Gobio insuyanus (En danger critique) n'a été observée que dans ces sources et dans la rivière associée à celles-ci. Comme dans une grande partie de la région, ce site est menacé par le captage excessif de l'eau, la régulation du débit de l'eau et la pollution. @ Jörg Freyhof

espèces de poissons sont endémiques à cette $Z C B$, dont deux hautement menacées en raison desquelles la ZCB répond aux critères d'admission en tant que site AZE. L'espèce de poisson Alburnus timarensis (En danger critique) est endémique à un unique cours d'eau (la rivière Karasu) se jetant dans le lac de Van, qui est affecté par la construction d'un barrage (à des fins d'irrigation et de maîtrise des inondations) et par les niveaux modérés de pollution domestique (Freyhof 2014c).

La loche de Van (Oxynoemacheilus ercisianus) est une espèce En danger et elle a été observée uniquement dans les trois rivières se jetant dans le lac de Van. Son aire de répartition a considérablement diminué suite à la construction de barrages (Freyhof 2014d).

Le système du Tigre et de l'Euphrate comporte 10 ZCB supplémentaires. En commençant à partir du delta, ces zones comprennent la ZCB du cours inférieur du Karun en Iran, un ensemble constitué de zones humides, de cours d'eau et de marais, et qui abrite sept espèces de déclenchement dont le barbeau Luciobarbus subquincunciatus (En danger critique). Trois ZCB englobent les marais du sud de l'Irak et de l'Iran : la ZCB des marais d'Hamar (est et ouest), la ZCB des marais centraux et la ZCB des marais d'Al Hawizah. Ces sites sont admis en tant que ZCB en raison de quatre espèces de poissons, dont Barbus grypus (Vulnérable), et de l'escargot endémique Gyraulus huwaizahensis (DD). Cinq ZCB concernant les affluents du Tigre en Irak sont admises en tant que ZCB surtout en raison des espèces de grands poissons largement répandues mais connaissant un déclin, comme le barbeau Luciobarbus esocinus (Vulnérable). Ces cinq sites sont la ZCB de Dalmaj, la ZCB de Sirvan - Shirvan - Dyala, la ZCB du cours inférieur du Petit Zab, la ZCB du cours supérieur du Petit Zab et la ZCB du Grand Zab. La seule ZCB située dans le bassin versant de l'Euphrate à l'extérieur du hotspot est la ZCB de l'Euphrate et du système karstique d'Haditha, qui est admise en tant que ZCB en raison de la population en déclin des espèces de grands poissons observées dans les ZCB du Tigre. Cette ZCB répond aussi aux critères d'admission en tant que site $A Z E$ (Système karstique d'Haditha) en raison de deux espèces de poissons cavernicoles En danger critique, Caecocypris basimi et Typhlogarra widdowsoni, qui sont menacés par le captage de l'eau et la baisse de niveau des eaux souterraines due aux modifications hydrologiques provoquées par la construction d'un grand barrage à proximité sur l'Euphrate (Freyhof 2014e,f).

Deux ZCB supplémentaires sont situées en dehors des trois zones décrites plus haut : i) la ZCB d'Ereğli et de Düzce en mer Noire, qui est un système côtier de grottes, de rivières et de sources proches de la côte de la mer Noire, et ; ii) la ZCB des zones humides d'Azraq dans le nord de la Jordanie. La ZCB d'Ereğli et de Düzce en mer Noire répond aussi aux critères d'admission en tant que site AZE en raison de deux espèces : la loche épineuse Cobitis splendens (En danger critique), qui est endémique à une seule petite rivière (polluée) au sein de la ZCB, et le gastéropode Belgrandiella cavernica (En danger critique 
(Peut-être éteint)), endémique au réseau de grottes d'Hercule, qui subit les effets du tourisme et n'a pas été enregistré depuis 60 ans (Freyhof 2014g ; Kebapçı et Seddon 2014). La ZCB des zones humides d'Azraq est admise en tant que ZCB en raison d'une seule espèce de poisson, Aphanius sirhani (En danger critique), qui est une espèce endémique. Les zones humides s'étaient complètement asséchées en 1992 (en raison du captage excessif de l'eau) mais elles ont depuis été restaurées, retrouvant jusqu'à $8 \%$ de leur ancienne superficie. Toutefois, les zones humides sont aujourd'hui complètement dépendantes d'une alimentation artificielle en eau car le captage de l'eau de manière continue a fait baisser la nappe phréatique, asséchant les sources. Aphanius sirhani a fait l'objet d'une reproduction en captivité et a été réintroduit avec succès dans les zones humides restaurées (Freyhof et Harrison 2014).

Post-scriptum : Le ministère de l'Environnement et de la Nature irakien vient de publier un rapport sur les Zones clés pour la biodiversité en Irak (Iraqi Ministry of Environment and Nature Iraq 2014). Sans surprise, il existe un chevauchement entre certaines ZCB figurant dans le rapport irakien et les ZCB d'eau douce identifiées dans le cadre de notre propre étude. D'autres sites identifiés ici s'ajoutent à la liste de ceux figurant dans l'étude irakienne. Les informations présentées dans notre rapport seront donc mises à la disposition des parties impliquées dans l'étude irakienne, afin d'inclure toute nouvelle ZCB d'eau douce, d'harmoniser les limites géographiques des ZCB en cas de chevauchement des sites identifiés au cours de ces deux processus, et de prendre note des actions prioritaires identifiées dans le cadre de ces études.

\section{Références}

BirdLife International (2014). Important Bird Areas factsheet: Tuz Lake. Downloaded from http://www.birdlife.org on 07/10/2014.

Crivelli, A.J. (2006). Salmo platycephalus. The IUCN Red List of Threatened Species. Version 2014.2. <www.iucnredlist.org>. Downloaded on 07 October 2014.

Freyhof, J. (2014a). Gobio gymnostethus. The IUCN Red List of Threatened Species. Version 2014.2. <www.iucnredlist.org>. Downloaded on 07 October 2014.

Freyhof, J. (2014b). Oxynoemacheilus seyhanensis. The IUCN Red List of Threatened Species. Version 2014.2. <www.iucnredlist.org>. Downloaded on 07 October 2014.

Freyhof, J. (2014c). Alburnus timarensis. The IUCN Red List of Threatened Species. Version 2014.2. <www.iucnredlist.org>. Downloaded on 07 October 2014.

Freyhof, J. (2014d). Oxynoemacheilus ercisianus. The IUCN Red List of Threatened Species. Version 2014.2. <www.iucnredlist.org>. Downloaded on 07 October 2014.

Freyhof, J. (2014e). Caecocypris basimi. The IUCN Red List of Threatened Species. Version 2014.2. <www.iucnredlist.org>. Downloaded on 07 October 2014.

Freyhof, J. (2014f). Typhlogarra widdowsoni. The IUCN Red List of Threatened Species. Version 2014.2. <www.iucnredlist.org>. Downloaded on 07 October 2014.

Freyhof, J. (2014g). Cobitis splendens. The IUCN Red List of Threatened Species. Version 2014.2. <www.iucnredlist.org>. Downloaded on 07 October 2014.

Freyhof, J. and Harrison, I.J. (2014). Aphanius sirhani. The IUCN Red List of Threatened Species. Version 2014.2. <www.iucnredlist.org>. Downloaded on 07 October 2014.

Iraqi Ministry of Environment and Nature Iraq (2014). Inventory of Key Biodiversity Areas of Iraq. Baghdad, Iraq: Iraqi Ministry of Environment and Nature Iraq.

Kebapçı, U. and Seddon, M.B. (2014). Belgrandiella cavernica. The IUCN Red List of Threatened Species. Version 2014.2. <www.iucnredlist. org>. Downloaded on 07 October 2014 


\section{Annexe IV. Espèces de déclenchement de ZCB}

Tableau A : Espèces de déclenchement validées dans les Balkans. C1 (Espèces menacées : CR En danger critique, EN En danger, VU Vulnérable) ; C2 (Aire de répartition restreinte) ; C3 (Communauté restreinte à un biome). * Sites AZE.

NA : non applicable.

Sous-région Nom de la $\mathrm{ZCB}^{1}$

\begin{tabular}{|ll}
\hline Balkans & Acheron $^{*}$ \\
\hline Balkans & Acheron $^{*}$ \\
\hline Balkans & Acheron $^{*}$ \\
\hline Balkans & Acheron $^{*}$ \\
\hline Balkans & Aggitis $^{*}$
\end{tabular}

\begin{tabular}{ll}
\hline Balkans & Aggitis* $^{*}$ \\
\hline Balkans & Aggitis $^{*}$ \\
\hline Balkans & Aggitis* $^{*}$
\end{tabular}

\begin{tabular}{llcl}
\hline Balkans & Aggitis* $^{*}$ & Phor \\
\hline Balkans & Aggitis* $^{*}$ & Turc
\end{tabular}

\begin{tabular}{ll}
\hline Balkans & Aggitis* $^{*}$ \\
\hline Balkans & Aliakmon Naoussa* \\
\hline Balkans & Andros Tinos
\end{tabular}

\begin{tabular}{ll}
\hline Balkans & Arachthos \\
\hline Balkans & Arachthos
\end{tabular}

\begin{tabular}{|lll}
\hline Balkans & Arachthos & Eco \\
\hline Balkans & Arachthos & Pela \\
\hline Balkans & Arachthos & Squ
\end{tabular}

\begin{tabular}{ll}
\hline Balkans & Arachthos \\
\hline Balkans & Arkadia Plateau
\end{tabular}

\begin{tabular}{|lll}
\hline Balkans & Arkadia Plateau & P \\
\hline Balkans & Arkadia Plateau & Squ \\
\hline Balkans & Arkadia Plateau & Squ \\
\hline
\end{tabular}

\begin{tabular}{lll} 
Balkans & Arkadia Plateau & Psen \\
\hline Balkans & Arkadia Plateau
\end{tabular}

\begin{tabular}{lll} 
& Arkadia Plateau \\
\hline Balkans & Butrint
\end{tabular}

\begin{tabular}{|ll}
\hline Balkans & Butrint \\
\hline Balkans & Butrint
\end{tabular}

\begin{tabular}{|ll}
\hline Balkans & Butrint \\
\hline Balkans & Butrint \\
\hline Bakans & Catchmentsurounding Niksic
\end{tabular}

\begin{tabular}{lll} 
Balkans & Catchment surrounding Niksic \\
\hline Balkans & Catchment surrounding Niksic
\end{tabular}

\begin{tabular}{|lll|}
\hline Balkans & Catchment surrounding Niksic & \\
\hline Balkans & Catchment surrounding Niksic & \\
\hline Balkans & Catchment surrounding Niksic & \\
\hline
\end{tabular}

\begin{tabular}{|ll|}
\hline Balkans & Catchment surrounding Niksic* \\
\hline Balkans & Catchment surrounding Niksic* \\
\hline Balkans & Cetina river
\end{tabular}

\begin{tabular}{ll} 
Balkans & Cetina river \\
\hline Balkans & Cetina river
\end{tabular}

\begin{tabular}{|lllllll}
\hline Balkans & Cetina river & Chondrostoma phoxinus & Poissons & EN & oui & NA \\
\hline Balkans & Cetina river & Phoxinellus alepidotus & Poissons & EN & oui & NA \\
\hline Balkans & Cetina river & Salmo obtusirostris & Poissons & EN & NA & NA \\
\hline Balkans & Cetina river & Scardinius dergle & Poissons & NA & oui & NA \\
\hline Balkans & Cetina river & Squalius illyricus & Poissons & NA & oui & NA \\
\hline Balkans & Cetina river & Squalius tenellus & Poissons & EN & oui & NA \\
\hline Balkans & Cetina river & Bithynia cettinensis & Mollusques & VU & oui & NA \\
\hline Balkans & Cetina river & Congeria kusceri & Mollusques & VU & NA & NA \\
\hline Balkans & Cetina river & Hauffenia jadertina & Mollusques & EN & oui & NA \\
\hline Balkans & Cetina river & Horatia klecakiana & Mollusques & NA & oui & NA \\
\hline
\end{tabular}


Tableau A : Espèces de déclenchement validées dans les Balkans (suite).

\begin{tabular}{|c|c|c|c|c|c|c|}
\hline Sous-région & Nom de la ZCB ${ }^{1}$ & Espèces de déclenchement & Groupe & C1 & $\mathrm{C}_{2}$ & C3 \\
\hline Balkans & Cetina river & Lanzaia elephantotus & Mollusques & NA & oui & NA \\
\hline Balkans & Cetina river & Lanzaia kotlusae & Mollusques & VU & oui & NA \\
\hline Balkans & Cetina river & Damasonium polyspermum & Plantes & VU & NA & NA \\
\hline Balkans & Corfu Island (Kerkyra) & Knipowitschia goerneri & Poissons & NA & oui & NA \\
\hline Balkans & Corfu Island (Kerkyra) & Pelasgus thesproticus & Poissons & NA & oui & NA \\
\hline Balkans & Corfu Island (Kerkyra) & Iglica sidariensis & Mollusques & VU & oui & NA \\
\hline Balkans & Corfu Island (Kerkyra) & Pseudamnicola macrostoma & Mollusques & NA & oui & NA \\
\hline Balkans & Corfu Island (Kerkyra) & Ceriagrion georgifreyi & Odonates & VU & NA & NA \\
\hline Balkans & Corfu Island (Kerkyra) & Pyrrhosoma elisabethae & Odonates & CR & oui & NA \\
\hline Balkans & Crete Central South & Bithynia candiota & Mollusques & NA & oui & NA \\
\hline Balkans & Crete Central South & Bythinella cretensis & Mollusques & NA & oui & NA \\
\hline Balkans & Crete Central South & Boyeria cretensis & Odonates & EN & oui & NA \\
\hline Balkans & Crete Central South & Coenagrion intermedium & Odonates & VU & oui & NA \\
\hline Balkans & Crete Central South & Carex troodi & Plantes & NA & oui & NA \\
\hline Balkans & Crete Eastern & Bithynia candiota & Mollusques & NA & oui & NA \\
\hline Balkans & Crete Eastern & Bythinella cretensis & Mollusques & NA & oui & NA \\
\hline Balkans & Crete Eastern & Boyeria cretensis & Odonates & EN & oui & NA \\
\hline Balkans & Crete Eastern & Coenagrion intermedium & Odonates & VU & oui & NA \\
\hline Balkans & Crete Eastern & Carex troodi & Plantes & NA & oui & NA \\
\hline Balkans & Crete North-west & Bithynia candiota & Mollusques & NA & oui & NA \\
\hline Balkans & Crete North-west & Bythinella cretensis & Mollusques & NA & oui & NA \\
\hline Balkans & Crete North-west & Boyeria cretensis & Odonates & EN & oui & NA \\
\hline Balkans & Crete North-west & Coenagrion intermedium & Odonates & VU & oui & NA \\
\hline Balkans & Crete North-west & Carex cretica & Plantes & NA & oui & NA \\
\hline Balkans & Crete North-west & Carex troodi & Plantes & NA & oui & NA \\
\hline Balkans & Crete South-west* & Bythinella cretensis & Mollusques & NA & oui & NA \\
\hline Balkans & Crete South-west* & Boyeria cretensis & Odonates & EN & oui & NA \\
\hline Balkans & Crete South-west* & Coenagrion intermedium & Odonates & VU & oui & NA \\
\hline Balkans & Crete South-west* & Callitriche pulchra & Plantes & CR & NA & NA \\
\hline Balkans & Crete South-west* & Carex cretica & Plantes & NA & oui & NA \\
\hline Balkans & Crete South-west* & Carex troodi & Plantes & NA & oui & NA \\
\hline Balkans & Chios & Pseudamnicola chia & Mollusques & VU & oui & NA \\
\hline Balkans & Doirani* & Alburnus macedonicus & Poissons & CR & oui & NA \\
\hline Balkans & Doirani* & Dreissena presbensis & Mollusques & NA & oui & NA \\
\hline Balkans & $\begin{array}{l}\text { Dragonija drainage in Slovenia and Croatia, Reka River in Slovenia } \\
\text { and Timavo spring, north of Trieste in Italy }\end{array}$ & Cottus scaturigo & Poissons & VU & oui & NA \\
\hline Balkans & $\begin{array}{l}\text { Dragonija drainage in Slovenia and Croatia, Reka River in Slovenia } \\
\text { and Timavo spring, north of Trieste in Italy }\end{array}$ & Chondrostoma soetta & Poissons & EN & NA & NA \\
\hline Balkans & $\begin{array}{l}\text { Dragonija drainage in Slovenia and Croatia, Reka River in Slovenia } \\
\text { and Timavo spring, north of Trieste in Italy }\end{array}$ & Romanogobio benacensis & Poissons & EN & NA & NA \\
\hline Balkans & $\begin{array}{l}\text { Dragonija drainage in Slovenia and Croatia, Reka River in Slovenia } \\
\text { and Timavo spring, north of Trieste in Italy }\end{array}$ & Squalius janae & Poissons & VU & oui & NA \\
\hline Balkans & $\begin{array}{l}\text { Dragonija drainage in Slovenia and Croatia, Reka River in Slovenia } \\
\text { and Timavo spring, north of Trieste in Italy }\end{array}$ & Acroloxus tetensi & Mollusques & VU & oui & NA \\
\hline Balkans & $\begin{array}{l}\text { Dragonija drainage in Slovenia and Croatia, Reka River in Slovenia } \\
\text { and Timavo spring, north of Trieste in Italy }\end{array}$ & Belgrandiella crucis & Mollusques & VU & oui & NA \\
\hline Balkans & $\begin{array}{l}\text { Dragonija drainage in Slovenia and Croatia, Reka River in Slovenia } \\
\text { and Timavo spring, north of Trieste in Italy }\end{array}$ & Belgrandiella schleschi & Mollusques & VU & oui & NA \\
\hline Balkans & $\begin{array}{l}\text { Dragonija drainage in Slovenia and Croatia, Reka River in Slovenia } \\
\text { and Timavo spring, north of Trieste in Italy }\end{array}$ & Belgrandiella superior & Mollusques & VU & oui & NA \\
\hline
\end{tabular}


Tableau A : Espèces de déclenchement validées dans les Balkans (suite).

\begin{tabular}{|c|c|c|c|c|c|c|}
\hline Sous-région & Nom de la ZCB ${ }^{1}$ & Espèces de déclenchement & Groupe & C1 & $\mathrm{C2}$ & C3 \\
\hline Balkans & $\begin{array}{l}\text { Dragonija drainage in Slovenia and Croatia, Reka River in Slovenia } \\
\text { and Timavo spring, north of Trieste in Italy }\end{array}$ & Iglica forumjuliana & Mollusques & NA & oui & NA \\
\hline Balkans & $\begin{array}{l}\text { Dragonija drainage in Slovenia and Croatia, Reka River in Slovenia } \\
\text { and Timavo spring, north of Trieste in Italy }\end{array}$ & Iglica giustii & Mollusques & NA & oui & NA \\
\hline Balkans & $\begin{array}{l}\text { Dragonija drainage in Slovenia and Croatia, Reka River in Slovenia } \\
\text { and Timavo spring, north of Trieste in Italy }\end{array}$ & Iglica hauffeni & Mollusques & NA & oui & NA \\
\hline Balkans & $\begin{array}{l}\text { Dragonija drainage in Slovenia and Croatia, Reka River in Slovenia } \\
\text { and Timavo spring, north of Trieste in Italy }\end{array}$ & Iglica tellinii & Mollusques & VU & oui & NA \\
\hline Balkans & $\begin{array}{l}\text { Dragonija drainage in Slovenia and Croatia, Reka River in Slovenia } \\
\text { and Timavo spring, north of Trieste in Italy }\end{array}$ & Istriana mirnae & Mollusques & NA & oui & NA \\
\hline Balkans & $\begin{array}{l}\text { Dragonija drainage in Slovenia and Croatia, Reka River in Slovenia } \\
\text { and Timavo spring, north of Trieste in Italy }\end{array}$ & Microcondylaea bonellii & Mollusques & VU & NA & NA \\
\hline Balkans & $\begin{array}{l}\text { Dragonija drainage in Slovenia and Croatia, Reka River in Slovenia } \\
\text { and Timavo spring, north of Trieste in Italy }\end{array}$ & Phreatica bolei & Mollusques & NA & oui & NA \\
\hline Balkans & $\begin{array}{l}\text { Dragonija drainage in Slovenia and Croatia, Reka River in Slovenia } \\
\text { and Timavo spring, north of Trieste in Italy }\end{array}$ & Plagigeyeria stochi & Mollusques & VU & oui & NA \\
\hline Balkans & $\begin{array}{l}\text { Dragonija drainage in Slovenia and Croatia, Reka River in Slovenia } \\
\text { and Timavo spring, north of Trieste in Italy }\end{array}$ & Vinodolia fiumana & Mollusques & EN & oui & NA \\
\hline Balkans & $\begin{array}{l}\text { Dragonija drainage in Slovenia and Croatia, Reka River in Slovenia } \\
\text { and Timavo spring, north of Trieste in Italy }\end{array}$ & Vinodolia fluviatilis & Mollusques & EN & NA & NA \\
\hline Balkans & $\begin{array}{l}\text { Dragonija drainage in Slovenia and Croatia, Reka River in Slovenia } \\
\text { and Timavo spring, north of Trieste in Italy }\end{array}$ & Damasonium polyspermum & Plantes & VU & NA & NA \\
\hline Balkans & Eastern Attica & Pelasgus marathonicus & Poissons & NA & oui & NA \\
\hline Balkans & Euboea Manikiatis* & Barbus euboicus & Poissons & CR & oui & NA \\
\hline Balkans & Euboea Manikiatis* & Squalius sp. nov. 'Evia' & Poissons & CR & oui & NA \\
\hline Balkans & Euboea Manikiatis* & Clameia brooki & Mollusques & NA & oui & NA \\
\hline Balkans & Evrotas & Pelasgus laconicus & Poissons & $\mathrm{CR}$ & oui & NA \\
\hline Balkans & Evrotas & Squalius keadicus & Poissons & EN & oui & NA \\
\hline Balkans & Evrotas & Tropidophoxinellus spartiaticus & Poissons & VU & oui & NA \\
\hline Balkans & Evrotas & Pseudamnicola exilis & Mollusques & NA & oui & NA \\
\hline Balkans & Evrotas - Arniotikos & Pseudamnicola exilis & Mollusques & NA & oui & NA \\
\hline Balkans & Evrotas - Gytheio & Squalius keadicus & Poissons & EN & oui & NA \\
\hline Balkans & Evrotas - Gytheio & Tropidophoxinellus spartiaticus & Poissons & VU & oui & NA \\
\hline Balkans & Evrotas - Gytheio & Pseudamnicola exilis & Mollusques & NA & oui & NA \\
\hline Balkans & Ismaris-Vosvozis-Filiouris & Anguilla anguilla & Poissons & CR & NA & NA \\
\hline Balkans & Ismaris-Vosvozis-Filiouris & Cyprinus carpio & Poissons & VU & NA & NA \\
\hline Balkans & Ismaris-Vosvozis-Filiouris & Unio crassus & Mollusques & EN & NA & NA \\
\hline Balkans & Kalamas & Economidichthys pygmaeus & Poissons & NA & oui & NA \\
\hline Balkans & Kalamas & Pelasgus thesproticus & Poissons & NA & oui & NA \\
\hline Balkans & Kalamas & Squalius pamvoticus & Poissons & NA & oui & NA \\
\hline Balkans & Kalamas & Valencia letourneuxi & Poissons & $\mathrm{CR}$ & oui & NA \\
\hline Balkans & Kalamas & Pyrrhosoma elisabethae & Odonates & CR & oui & NA \\
\hline Balkans & Karla* & Barbus sperchiensis & Poissons & NA & oui & NA \\
\hline Balkans & Karla* & Cobitis stephanidisi & Poissons & CR & oui & NA \\
\hline Balkans & Karla* & Knipowitschia thessala & Poissons & EN & oui & NA \\
\hline Balkans & Karla* & Daphniola exigua & Mollusques & EN & oui & NA \\
\hline Balkans & Karpathos & Pseudamnicola pieperi & Mollusques & VU & oui & NA \\
\hline Balkans & Kastoria & Salmo pelagonicus & Poissons & VU & oui & NA \\
\hline Balkans & Kastoria & Bithynia kastorias & Mollusques & $\mathrm{CR}$ & oui & NA \\
\hline Balkans & Kastraki & Cobitis trichonica & Poissons & EN & oui & NA \\
\hline Balkans & Kastraki & Economidichthys pygmaeus & Poissons & NA & oui & NA \\
\hline
\end{tabular}


Tableau A : Espèces de déclenchement validées dans les Balkans (suite).

\begin{tabular}{|c|c|c|c|c|c|c|}
\hline Sous-région & Nom de la ZCB ${ }^{1}$ & Espèces de déclenchement & Groupe & C1 & $\mathrm{C} 2$ & C3 \\
\hline Balkans & Kastraki & Pelasgus stymphalicus & Poissons & NA & oui & NA \\
\hline Balkans & Kastraki & Scardinius acarnanicus & Poissons & NA & oui & NA \\
\hline Balkans & Kastraki & Silurus aristotelis & Poissons & NA & oui & NA \\
\hline Balkans & Kastraki & Tropidophoxinellus hellenicus & Poissons & NA & oui & NA \\
\hline Balkans & Kastraki & Belgrandiella haesitans & Mollusques & NA & oui & NA \\
\hline Balkans & Kastraki & Dianella thiesseana & Mollusques & CR & oui & NA \\
\hline Balkans & Kastraki & Dreissena blanci & Mollusques & VU & oui & NA \\
\hline Balkans & Kastraki & Islamia trichoniana & Mollusques & CR & oui & NA \\
\hline Balkans & Kastraki & Pseudobithynia falniowskii & Mollusques & CR & oui & NA \\
\hline Balkans & Kastraki & Pseudobithynia panetolis & Mollusques & CR & oui & NA \\
\hline Balkans & Kastraki & Pseudobithynia trichonis & Mollusques & EN & oui & NA \\
\hline Balkans & Kastraki & Pseudoislamia balcanica & Mollusques & CR & oui & NA \\
\hline Balkans & Kastraki & Valvata klemmi & Mollusques & EN & oui & NA \\
\hline Balkans & Kephalonia and Ithaki & Fissuria raehlei & Mollusques & NA & oui & NA \\
\hline Balkans & Kerkini & Alburnus sp. nov. 'Volvi' & Poissons & NA & oui & NA \\
\hline Balkans & Krka drainage & Aulopyge huegelii & Poissons & EN & oui & NA \\
\hline Balkans & Krka drainage & Knipowitschia mrakovcici & Poissons & CR & oui & NA \\
\hline Balkans & Krka drainage & Phoxinellus dalmaticus & Poissons & CR & oui & NA \\
\hline Balkans & Krka drainage & Salmo obtusirostris & Poissons & EN & NA & NA \\
\hline Balkans & Krka drainage & Scardinius dergle & Poissons & NA & oui & NA \\
\hline Balkans & Krka drainage & Squalius illyricus & Poissons & NA & oui & NA \\
\hline Balkans & Krka drainage & Squalius zrmanjae & Poissons & NA & oui & NA \\
\hline Balkans & Krka drainage & Telestes turskyi & Poissons & CR & oui & NA \\
\hline Balkans & Krka drainage & Congeria kusceri & Mollusques & VU & NA & NA \\
\hline Balkans & Krka drainage & Dalmatella sketi & Mollusques & $\mathrm{CR}$ & oui & NA \\
\hline Balkans & Krka drainage & Hadziella sketi & Mollusques & VU & oui & NA \\
\hline Balkans & Krka drainage & Lanzaia skradinensis & Mollusques & $\mathrm{CR}$ & oui & NA \\
\hline Balkans & Krka drainage & Pseudobithynia kirka & Mollusques & VU & oui & NA \\
\hline Balkans & Krka drainage & Damasonium polyspermum & Plantes & VU & NA & NA \\
\hline Balkans & Ladon & Pelasgus stymphalicus & Poissons & NA & oui & NA \\
\hline Balkans & Ladon & Squalius peloponnensis & Poissons & NA & oui & NA \\
\hline Balkans & Ladon & Hauffenia edlingeri & Mollusques & $\mathrm{CR}$ & oui & NA \\
\hline Balkans & Ladon & Pyrrhosoma elisabethae & Odonates & $\mathrm{CR}$ & oui & NA \\
\hline Balkans & Lake Bilecko & Cobitis narentana & Poissons & VU & oui & NA \\
\hline Balkans & Lake Bilecko & Delminichthys ghetaldii & Poissons & VU & oui & NA \\
\hline Balkans & Lake Bilecko & Squalius svallize & Poissons & $\mathrm{CR}$ & oui & NA \\
\hline Balkans & Lake Bilecko & Antibaria notata & Mollusques & NA & oui & NA \\
\hline Balkans & Lake Bilecko & Belgrandia torifera & Mollusques & VU & oui & NA \\
\hline Balkans & Lake Bilecko & Congeria kusceri & Mollusques & VU & NA & NA \\
\hline Balkans & Lake Bilecko & Emmericia expansilabris & Mollusques & VU & oui & NA \\
\hline Balkans & Lake Bilecko & Emmericia ventricosa & Mollusques & VU & oui & NA \\
\hline Balkans & Lake Bilecko & Iglica absoloni & Mollusques & NA & oui & NA \\
\hline Balkans & Lake Bilecko & Iglica bagliviaeformis & Mollusques & EN & NA & NA \\
\hline Balkans & Lake Bilecko & Lanzaia vjetrenicae & Mollusques & VU & oui & NA \\
\hline Balkans & Lake Bilecko & Narentiana vjetrenicae & Mollusques & EN & oui & NA \\
\hline Balkans & Lake Bilecko & Paladilhiopsis pretneri & Mollusques & NA & oui & NA \\
\hline Balkans & Lake Bilecko & Paladilhiopsis solida & Mollusques & NA & oui & NA \\
\hline
\end{tabular}


Tableau A : Espèces de déclenchement validées dans les Balkans (suite).

\begin{tabular}{|c|c|c|c|c|c|c|}
\hline Sous-région & Nom de la ZCB' ${ }^{1}$ & Espèces de déclenchement & Groupe & C1 & $\mathrm{C}_{2}$ & C3 \\
\hline Balkans & Lake Bilecko & Plagigeyeria gladilini & Mollusques & VU & NA & NA \\
\hline Balkans & Lake Bilecko & Plagigeyeria tribunicae & Mollusques & VU & oui & NA \\
\hline Balkans & Lake Bilecko & Saxurinator brandti & Mollusques & VU & NA & NA \\
\hline Balkans & Lake Bilecko & Saxurinator labiatus & Mollusques & $\mathrm{CR}$ & oui & NA \\
\hline Balkans & Lake Bilecko & Saxurinator montenegrinus & Mollusques & EN & oui & NA \\
\hline Balkans & Lake Bilecko & Vinodolia fluviatilis & Mollusques & EN & NA & NA \\
\hline Balkans & Lake Bilecko & Vinodolia hadouphylax & Mollusques & $\mathrm{CR}$ & oui & NA \\
\hline Balkans & Lake Busko & Aulopyge huegelii & Poissons & EN & oui & NA \\
\hline Balkans & Lake Busko & Cobitis dalmatina & Poissons & VU & oui & NA \\
\hline Balkans & Lake Busko & Chondrostoma phoxinus & Poissons & EN & oui & NA \\
\hline Balkans & Lake Busko & Delminichthys adspersus & Poissons & VU & oui & NA \\
\hline Balkans & Lake Busko & Delminichthys ghetaldii & Poissons & VU & oui & NA \\
\hline Balkans & Lake Busko & Phoxinellus alepidotus & Poissons & EN & oui & NA \\
\hline Balkans & Lake Busko & Rutilus basak & Poissons & NA & oui & NA \\
\hline Balkans & Lake Busko & Salmo obtusirostris & Poissons & EN & NA & NA \\
\hline Balkans & Lake Busko & Scardinius dergle & Poissons & NA & oui & NA \\
\hline Balkans & Lake Busko & Scardinius plotizza & Poissons & NA & oui & NA \\
\hline Balkans & Lake Busko & Squalius illyricus & Poissons & NA & oui & NA \\
\hline Balkans & Lake Busko & Squalius tenellus & Poissons & EN & oui & NA \\
\hline Balkans & Lake Busko & Antibaria notata & Mollusques & NA & oui & NA \\
\hline Balkans & Lake Busko & Belgrandia torifera & Mollusques & VU & oui & NA \\
\hline Balkans & Lake Busko & Congeria kusceri & Mollusques & VU & NA & NA \\
\hline Balkans & Lake Busko & Emmericia expansilabris & Mollusques & VU & oui & NA \\
\hline Balkans & Lake Busko & Emmericia ventricosa & Mollusques & VU & oui & NA \\
\hline Balkans & Lake Busko & Iglica absoloni & Mollusques & NA & oui & NA \\
\hline Balkans & Lake Busko & Iglica bagliviaeformis & Mollusques & EN & NA & NA \\
\hline Balkans & Lake Busko & Lanzaia kotlusae & Mollusques & VU & oui & NA \\
\hline Balkans & Lake Busko & Paladilhiopsis pretneri & Mollusques & NA & oui & NA \\
\hline Balkans & Lake Busko & Plagigeyeria gladilini & Mollusques & VU & NA & NA \\
\hline Balkans & Lake Busko & Saxurinator brandti & Mollusques & VU & NA & NA \\
\hline Balkans & Lake Busko & Saxurinator labiatus & Mollusques & CR & oui & NA \\
\hline Balkans & Lake Busko & Saxurinator montenegrinus & Mollusques & EN & oui & NA \\
\hline Balkans & Lake Busko & Vinodolia fluviatilis & Mollusques & EN & NA & NA \\
\hline Balkans & Lake Busko & Damasonium polyspermum & Plantes & VU & NA & NA \\
\hline Balkans & Lake Kastrakiou & Cobitis trichonica & Poissons & EN & oui & NA \\
\hline Balkans & Lake Kastrakiou & Economidichthys pygmaeus & Poissons & NA & oui & NA \\
\hline Balkans & Lake Kastrakiou & Pelasgus stymphalicus & Poissons & NA & oui & NA \\
\hline Balkans & Lake Kastrakiou & Rutilus panosi & Poissons & VU & oui & NA \\
\hline Balkans & Lake Kastrakiou & Scardinius acarnanicus & Poissons & NA & oui & NA \\
\hline Balkans & Lake Kastrakiou & Silurus aristotelis & Poissons & NA & oui & NA \\
\hline Balkans & Lake Kastrakiou & Tropidophoxinellus hellenicus & Poissons & NA & oui & NA \\
\hline Balkans & Lake Kastrakiou & Belgrandiella haesitans & Mollusques & NA & oui & NA \\
\hline Balkans & Lake Kastrakiou & Dianella thiesseana & Mollusques & $\mathrm{CR}$ & oui & NA \\
\hline Balkans & Lake Kastrakiou & Dreissena blanci & Mollusques & VU & oui & NA \\
\hline Balkans & Lake Kastrakiou & Islamia trichoniana & Mollusques & CR & oui & NA \\
\hline Balkans & Lake Kastrakiou & Pseudobithynia falniowskii & Mollusques & $\mathrm{CR}$ & oui & NA \\
\hline Balkans & Lake Kastrakiou & Pseudobithynia panetolis & Mollusques & $\mathrm{CR}$ & oui & NA \\
\hline
\end{tabular}


Tableau A : Espèces de déclenchement validées dans les Balkans (suite).

\begin{tabular}{|c|c|c|c|c|c|c|}
\hline Sous-région & Nom de la ZCB' ${ }^{1}$ & Espèces de déclenchement & Groupe & C1 & $\mathrm{C}_{2}$ & C3 \\
\hline Balkans & Lake Kastrakiou & Pseudobithynia trichonis & Mollusques & EN & oui & NA \\
\hline Balkans & Lake Kastrakiou & Pseudoislamia balcanica & Mollusques & $\mathrm{CR}$ & oui & NA \\
\hline Balkans & Lake Kastrakiou & Valvata klemmi & Mollusques & EN & oui & NA \\
\hline Balkans & Lake Ohrid* & Alburnoides ohridanus & Poissons & VU & oui & oui \\
\hline Balkans & Lake Ohrid* & Alburnus scoranza & Poissons & NA & oui & NA \\
\hline Balkans & Lake Ohrid* & Barbatula sturanyi & Poissons & NA & oui & NA \\
\hline Balkans & Lake Ohrid* & Barbus rebeli & Poissons & NA & oui & NA \\
\hline Balkans & Lake Ohrid* & Gobio ohridanus & Poissons & VU & oui & oui \\
\hline Balkans & Lake Ohrid* & Pelasgus minutus & Poissons & NA & oui & NA \\
\hline Balkans & Lake Ohrid* & Rutilus karamani & Poissons & NA & oui & NA \\
\hline Balkans & Lake Ohrid* & Rutilus ohridanus & Poissons & NA & oui & NA \\
\hline Balkans & Lake Ohrid* & Salmo aphelios & Poissons & NA & oui & oui \\
\hline Balkans & Lake Ohrid* & Salmo balcanicus & Poissons & NA & oui & oui \\
\hline Balkans & Lake Ohrid* & Salmo letnica & Poissons & NA & oui & oui \\
\hline Balkans & Lake Ohrid* & Salmo lumi & Poissons & NA & oui & oui \\
\hline Balkans & Lake Ohrid* & Salmo ohridanus & Poissons & VU & oui & oui \\
\hline Balkans & Lake Ohrid* & Scardinius knezevici & Poissons & NA & oui & oui \\
\hline Balkans & Lake Ohrid* & Acroloxus improvisus & Mollusques & VU & oui & oui \\
\hline Balkans & Lake Ohrid* & Acroloxus macedonicus & Mollusques & $\mathrm{CR}$ & oui & oui \\
\hline Balkans & Lake Ohrid* & Ancylus lapicidus & Mollusques & EN & oui & oui \\
\hline Balkans & Lake Ohrid* & Ancylus scalariformis & Mollusques & VU & oui & oui \\
\hline Balkans & Lake Ohrid* & Ancylus tapirulus & Mollusques & EN & oui & oui \\
\hline Balkans & Lake Ohrid* & Chilopyrgula sturanyi & Mollusques & NA & oui & oui \\
\hline Balkans & Lake Ohrid* & Dreissena presbensis & Mollusques & NA & oui & NA \\
\hline Balkans & Lake Ohrid* & Ginaia munda & Mollusques & VU & oui & oui \\
\hline Balkans & Lake Ohrid* & Gocea ohridana & Mollusques & CR & oui & oui \\
\hline Balkans & Lake Ohrid* & Gyraulus albidus & Mollusques & VU & oui & oui \\
\hline Balkans & Lake Ohrid* & Gyraulus crenophilus & Mollusques & EN & oui & oui \\
\hline Balkans & Lake Ohrid* & Gyraulus fontinalis & Mollusques & EN & oui & oui \\
\hline Balkans & Lake Ohrid* & Gyraulus lychnidicus & Mollusques & NA & oui & oui \\
\hline Balkans & Lake Ohrid* & Gyraulus trapezoides & Mollusques & EN & oui & oui \\
\hline Balkans & Lake Ohrid* & Lyhnidia gjorgjevici & Mollusques & EN & oui & oui \\
\hline Balkans & Lake Ohrid* & Lyhnidia hadzii & Mollusques & CR & oui & oui \\
\hline Balkans & Lake Ohrid* & Lyhnidia karamani & Mollusques & CR & oui & oui \\
\hline Balkans & Lake Ohrid* & Lyhnidia stankovici & Mollusques & CR & oui & oui \\
\hline Balkans & Lake Ohrid* & Lyhnidia sublitoralis & Mollusques & NA & oui & oui \\
\hline Balkans & Lake Ohrid* & Micropyrgula stankovici & Mollusques & VU & oui & oui \\
\hline Balkans & Lake Ohrid* & Neofossarulus stankovici & Mollusques & VU & oui & oui \\
\hline Balkans & Lake Ohrid* & Ochridopyrgula macedonica & Mollusques & NA & oui & oui \\
\hline Balkans & Lake Ohrid* & Ohridohauffenia depressa & Mollusques & EN & oui & oui \\
\hline Balkans & Lake Ohrid* & Ohridohauffenia rotonda & Mollusques & EN & oui & oui \\
\hline Balkans & Lake Ohrid* & Ohridohauffenia sanctinaumi & Mollusques & EN & oui & oui \\
\hline Balkans & Lake Ohrid* & Ohridohauffenia sublitoralis & Mollusques & NA & oui & oui \\
\hline Balkans & Lake Ohrid* & Ohridohoratia carinata & Mollusques & EN & oui & oui \\
\hline Balkans & Lake Ohrid* & Ohridohoratia polinskii & Mollusques & VU & oui & oui \\
\hline Balkans & Lake Ohrid* & Ohridohoratia pygmaea & Mollusques & NA & oui & oui \\
\hline Balkans & Lake Ohrid* & Ohridohoratia sturanyi & Mollusques & NA & oui & oui \\
\hline
\end{tabular}


Tableau A : Espèces de déclenchement validées dans les Balkans (suite).

\begin{tabular}{|c|c|c|c|c|c|c|}
\hline Sous-région & Nom de la ZCB' ${ }^{1}$ & Espèces de déclenchement & Groupe & C1 & $\mathrm{C}_{2}$ & C3 \\
\hline Balkans & Lake Ohrid* & Ohrigocea karevi & Mollusques & EN & oui & oui \\
\hline Balkans & Lake Ohrid* & Ohrigocea miladinovorum & Mollusques & EN & oui & oui \\
\hline Balkans & Lake Ohrid* & Ohrigocea ornata & Mollusques & EN & oui & oui \\
\hline Balkans & Lake Ohrid* & Ohrigocea samuili & Mollusques & EN & oui & oui \\
\hline Balkans & Lake Ohrid* & Ohrigocea stankovici & Mollusques & EN & oui & oui \\
\hline Balkans & Lake Ohrid* & Pisidium edlaueri & Mollusques & EN & oui & oui \\
\hline Balkans & Lake Ohrid* & Planorbis macedonicus & Mollusques & EN & oui & oui \\
\hline Balkans & Lake Ohrid* & Pseudohoratia brusinae & Mollusques & VU & oui & oui \\
\hline Balkans & Lake Ohrid* & Pseudohoratia lacustris & Mollusques & VU & oui & oui \\
\hline Balkans & Lake Ohrid* & Pseudohoratia ochridana & Mollusques & VU & oui & oui \\
\hline Balkans & Lake Ohrid* & Pyrgohydrobia grochmalickii & Mollusques & VU & oui & oui \\
\hline Balkans & Lake Ohrid* & Pyrgohydrobia jablanicensis & Mollusques & CR & oui & oui \\
\hline Balkans & Lake Ohrid* & Pyrgohydrobia sanctinaumi & Mollusques & VU & oui & oui \\
\hline Balkans & Lake Ohrid* & Radix relicta & Mollusques & NA & oui & oui \\
\hline Balkans & Lake Ohrid* & Stankovicia baicaliiformis & Mollusques & CR & oui & oui \\
\hline Balkans & Lake Ohrid* & Stankovicia pavlovici & Mollusques & VU & oui & oui \\
\hline Balkans & Lake Ohrid* & Stankovicia wagneri & Mollusques & VU & oui & oui \\
\hline Balkans & Lake Ohrid* & Trachyochridia filocincta & Mollusques & CR & oui & oui \\
\hline Balkans & Lake Ohrid* & Valvata hirsutecostata & Mollusques & VU & oui & oui \\
\hline Balkans & Lake Ohrid* & Valvata relicta & Mollusques & VU & oui & oui \\
\hline Balkans & Lake Ohrid* & Valvata rhabdota & Mollusques & NA & oui & oui \\
\hline Balkans & Lake Ohrid* & Valvata stenotrema & Mollusques & NA & oui & oui \\
\hline Balkans & Lake Ohrid* & Xestopyrgula dybowskii & Mollusques & VU & oui & oui \\
\hline Balkans & Lake Ohrid* & Zaumia kusceri & Mollusques & CR & oui & oui \\
\hline Balkans & Lake Skadar* & Alburnus scoranza & Poissons & NA & oui & NA \\
\hline Balkans & Lake Skadar* & Alosa sp. nov. 'Skadar' & Poissons & VU & oui & NA \\
\hline Balkans & Lake Skadar* & Barbus rebeli & Poissons & NA & oui & NA \\
\hline Balkans & Lake Skadar* & Gobio skadarensis & Poissons & EN & oui & NA \\
\hline Balkans & Lake Skadar* & Pelasgus minutus & Poissons & NA & oui & NA \\
\hline Balkans & Lake Skadar* & Scardinius knezevici & Poissons & NA & oui & NA \\
\hline Balkans & Lake Skadar* & Telestes montenigrinus & Poissons & NA & oui & NA \\
\hline Balkans & Lake Skadar* & Bithynia hambergerae & Mollusques & NA & oui & NA \\
\hline Balkans & Lake Skadar* & Bithynia radomani & Mollusques & NA & oui & NA \\
\hline Balkans & Lake Skadar* & Bithynia skadarskii & Mollusques & EN & oui & NA \\
\hline Balkans & Lake Skadar* & Bithynia zeta & Mollusques & EN & oui & NA \\
\hline Balkans & Lake Skadar* & Bracenica spiridoni & Mollusques & EN & oui & NA \\
\hline Balkans & Lake Skadar* & Dreissena presbensis & Mollusques & NA & oui & NA \\
\hline Balkans & Lake Skadar* & Gyraulus meierbrooki & Mollusques & EN & oui & NA \\
\hline Balkans & Lake Skadar* & Plagigeyeria gladilini & Mollusques & VU & NA & NA \\
\hline Balkans & Lake Skadar* & Pyrgula annulata & Mollusques & NA & oui & NA \\
\hline Balkans & Lake Skadar* & Radix skutaris & Mollusques & EN & oui & NA \\
\hline Balkans & Lake Skadar* & Radomaniola elongata & Mollusques & CR & oui & NA \\
\hline Balkans & Lake Skadar* & Radomaniola lacustris & Mollusques & CR & oui & NA \\
\hline Balkans & Lake Skadar* & Stagnicola montenegrinus & Mollusques & NA & oui & NA \\
\hline Balkans & Lake Skadar* & Valvata montenegrina & Mollusques & EN & oui & NA \\
\hline Balkans & Lake Skadar* & Vinodolia matjasici & Mollusques & CR & oui & NA \\
\hline Balkans & Lake Skadar* & Vinodolia scutarica & Mollusques & EN & oui & NA \\
\hline
\end{tabular}


Tableau A : Espèces de déclenchement validées dans les Balkans (suite).

\begin{tabular}{|c|c|c|c|c|c|c|}
\hline Sous-région & Nom de la ZCB ${ }^{1}$ & Espèces de déclenchement & Groupe & C1 & C2 & C3 \\
\hline Balkans & Lake Skadar* & Pilularia minuta & Plantes & EN & NA & NA \\
\hline Balkans & $\begin{array}{l}\text { Lakes Limnothalassa Rodias, Limnothalassa Tsoukaliou, } \\
\text { Limnothalassa Lagarou }\end{array}$ & Cobitis arachthosensis & Poissons & EN & oui & NA \\
\hline Balkans & $\begin{array}{l}\text { Lakes Limnothalassa Rodias, Limnothalassa Tsoukaliou, } \\
\text { Limnothalassa Lagarou }\end{array}$ & Cobitis hellenica & Poissons & EN & oui & NA \\
\hline Balkans & $\begin{array}{l}\text { Lakes Limnothalassa Rodias, Limnothalassa Tsoukaliou, } \\
\text { Limnothalassa Lagarou }\end{array}$ & Economidichthys pygmaeus & Poissons & NA & oui & NA \\
\hline Balkans & $\begin{array}{l}\text { Lakes Limnothalassa Rodias, Limnothalassa Tsoukaliou, } \\
\text { Limnothalassa Lagarou }\end{array}$ & Pelasgus thesproticus & Poissons & NA & oui & NA \\
\hline Balkans & $\begin{array}{l}\text { Lakes Limnothalassa Rodias, Limnothalassa Tsoukaliou, } \\
\text { Limnothalassa Lagarou }\end{array}$ & Squalius pamvoticus & Poissons & NA & oui & NA \\
\hline Balkans & $\begin{array}{l}\text { Lakes Limnothalassa Rodias, Limnothalassa Tsoukaliou, } \\
\text { Limnothalassa Lagarou }\end{array}$ & Valencia letourneuxi & Poissons & CR & oui & NA \\
\hline Balkans & $\begin{array}{l}\text { Lakes Limnothalassa Rodias, Limnothalassa Tsoukaliou, } \\
\text { Limnothalassa Lagarou }\end{array}$ & Belgrandiella haesitans & Mollusques & NA & oui & NA \\
\hline Balkans & Lakes Trichonis and Lisimachia* & Cobitis trichonica & Poissons & EN & oui & NA \\
\hline Balkans & Lakes Trichonis and Lisimachia* & Economidichthys pygmaeus & Poissons & NA & oui & NA \\
\hline Balkans & Lakes Trichonis and Lisimachia* & Economidichthys trichonis & Poissons & EN & oui & NA \\
\hline Balkans & Lakes Trichonis and Lisimachia* & Pelasgus stymphalicus & Poissons & NA & oui & NA \\
\hline Balkans & Lakes Trichonis and Lisimachia* & Rutilus panosi & Poissons & VU & oui & NA \\
\hline Balkans & Lakes Trichonis and Lisimachia* & Salaria economidisi & Poissons & CR & oui & NA \\
\hline Balkans & Lakes Trichonis and Lisimachia* & Scardinius acarnanicus & Poissons & NA & oui & NA \\
\hline Balkans & Lakes Trichonis and Lisimachia* & Silurus aristotelis & Poissons & NA & oui & NA \\
\hline Balkans & Lakes Trichonis and Lisimachia* & Tropidophoxinellus hellenicus & Poissons & NA & oui & NA \\
\hline Balkans & Lakes Trichonis and Lisimachia* & Belgrandiella haesitans & Mollusques & NA & oui & NA \\
\hline Balkans & Lakes Trichonis and Lisimachia* & Dianella thiesseana & Mollusques & CR & oui & NA \\
\hline Balkans & Lakes Trichonis and Lisimachia* & Dreissena blanci & Mollusques & VU & oui & NA \\
\hline Balkans & Lakes Trichonis and Lisimachia* & Islamia trichoniana & Mollusques & CR & oui & NA \\
\hline Balkans & Lakes Trichonis and Lisimachia* & Pseudobithynia falniowskii & Mollusques & CR & oui & NA \\
\hline Balkans & Lakes Trichonis and Lisimachia* & Pseudobithynia panetolis & Mollusques & CR & oui & NA \\
\hline Balkans & Lakes Trichonis and Lisimachia* & Pseudobithynia trichonis & Mollusques & EN & oui & NA \\
\hline Balkans & Lakes Trichonis and Lisimachia* & Pseudoislamia balcanica & Mollusques & CR & oui & NA \\
\hline Balkans & Lakes Trichonis and Lisimachia* & Valvata klemmi & Mollusques & EN & oui & NA \\
\hline Balkans & Lesvos & Squalius cii & Poissons & NA & oui & NA \\
\hline Balkans & Lesvos & Pilularia minuta & Plantes & EN & NA & NA \\
\hline Balkans & Listica river and Mostarsko blato & Alburnus neretvae & Poissons & NA & oui & NA \\
\hline Balkans & Listica river and Mostarsko blato & Delminichthys adspersus & Poissons & VU & oui & NA \\
\hline Balkans & Listica river and Mostarsko blato & Delminichthys ghetaldii & Poissons & VU & oui & NA \\
\hline Balkans & Listica river and Mostarsko blato & Phoxinellus pseudalepidotus & Poissons & VU & oui & NA \\
\hline Balkans & Listica river and Mostarsko blato & Rutilus basak & Poissons & NA & oui & NA \\
\hline Balkans & Listica river and Mostarsko blato & Scardinius plotizza & Poissons & NA & oui & NA \\
\hline Balkans & Listica river and Mostarsko blato & Congeria kusceri & Mollusques & VU & NA & NA \\
\hline Balkans & Listica river and Mostarsko blato & Paladilhiopsis solida & Mollusques & NA & oui & NA \\
\hline Balkans & Lower Acheloos & Cobitis trichonica & Poissons & EN & oui & NA \\
\hline Balkans & Lower Acheloos & Economidichthys pygmaeus & Poissons & NA & oui & NA \\
\hline Balkans & Lower Acheloos & Pelasgus stymphalicus & Poissons & NA & oui & NA \\
\hline Balkans & Lower Acheloos & Scardinius acarnanicus & Poissons & NA & oui & NA \\
\hline Balkans & Lower Acheloos & Silurus aristotelis & Poissons & NA & oui & NA \\
\hline Balkans & Lower Acheloos & Tropidophoxinellus hellenicus & Poissons & NA & oui & NA \\
\hline Balkans & Lower Acheloos & Valencia letourneuxi & Poissons & CR & oui & NA \\
\hline
\end{tabular}


Tableau A : Espèces de déclenchement validées dans les Balkans (suite).

\begin{tabular}{|c|c|c|c|c|c|c|}
\hline Sous-région & Nom de la ZCB ${ }^{1}$ & Espèces de déclenchement & Groupe & C1 & $\mathbf{C 2}$ & C3 \\
\hline Balkans & Lower Acheloos & Belgrandiella haesitans & Mollusques & NA & oui & NA \\
\hline Balkans & Lower Acheloos & Dianella thiesseana & Mollusques & CR & oui & NA \\
\hline Balkans & Lower Acheloos & Dreissena blanci & Mollusques & VU & oui & NA \\
\hline Balkans & Lower Acheloos & Islamia epirana & Mollusques & VU & oui & NA \\
\hline Balkans & Lower Acheloos & Islamia trichoniana & Mollusques & CR & oui & NA \\
\hline Balkans & Lower Acheloos & Pseudobithynia falniowskii & Mollusques & CR & oui & NA \\
\hline Balkans & Lower Acheloos & Pseudobithynia panetolis & Mollusques & CR & oui & NA \\
\hline Balkans & Lower Acheloos & Pseudobithynia trichonis & Mollusques & EN & oui & NA \\
\hline Balkans & Lower Acheloos & Valvata klemmi & Mollusques & EN & oui & NA \\
\hline Balkans & Lower Alfeios & Pelasgus stymphalicus & Poissons & NA & oui & NA \\
\hline Balkans & Lower Alfeios & Squalius peloponnensis & Poissons & NA & oui & NA \\
\hline Balkans & Lower Alfeios & Valencia letourneuxi & Poissons & CR & oui & NA \\
\hline Balkans & Lower Alfeios & Iglica wolfischeri & Mollusques & CR & oui & NA \\
\hline Balkans & Lower Axios & Zingel balcanicus & Poissons & NA & oui & NA \\
\hline Balkans & Lower Bojana river basin* & Alburnus scoranza & Poissons & NA & oui & NA \\
\hline Balkans & Lower Bojana river basin* & Pelasgus minutus & Poissons & NA & oui & NA \\
\hline Balkans & Lower Bojana river basin* & Rutilus karamani & Poissons & NA & oui & NA \\
\hline Balkans & Lower Bojana river basin* & Telestes montenigrinus & Poissons & NA & oui & NA \\
\hline Balkans & Lower Bojana river basin* & Bithynia radomani & Mollusques & NA & oui & NA \\
\hline Balkans & Lower Bojana river basin* & Gyraulus ioanis & Mollusques & CR & oui & NA \\
\hline Balkans & Lower Bojana river basin* & Gyraulus shasi & Mollusques & CR & oui & NA \\
\hline Balkans & Lower Bojana river basin* & Plagigeyeria gladilini & Mollusques & VU & NA & NA \\
\hline Balkans & Lower Bojana river basin* & Radomaniola montana & Mollusques & NA & oui & NA \\
\hline Balkans & Lower Bojana river basin* & Saxurinator brandti & Mollusques & VU & NA & NA \\
\hline Balkans & Lower Bojana river basin* & Vinodolia gluhodolica & Mollusques & EN & oui & NA \\
\hline Balkans & Lower Bojana river basin* & Pilularia minuta & Plantes & EN & NA & NA \\
\hline Balkans & Magnisia* & Barbus sperchiensis & Poissons & NA & oui & NA \\
\hline Balkans & Magnisia* & Pelasgus marathonicus & Poissons & NA & oui & NA \\
\hline Balkans & Magnisia* & Graecorientalia vrissiana & Mollusques & CR & oui & NA \\
\hline Balkans & Magnisia* & Heleobia tritonum & Mollusques & CR & oui & NA \\
\hline Balkans & Magnisia* & Turcorientalia hohenackeri & Mollusques & VU & oui & NA \\
\hline Balkans & Matica river and Bacina lakes & Alburnus neretvae & Poissons & NA & oui & NA \\
\hline Balkans & Matica river and Bacina lakes & Delminichthys adspersus & Poissons & VU & oui & NA \\
\hline Balkans & Matica river and Bacina lakes & Delminichthys ghetaldii & Poissons & VU & oui & NA \\
\hline Balkans & Matica river and Bacina lakes & Rutilus basak & Poissons & NA & oui & NA \\
\hline Balkans & Matica river and Bacina lakes & Salmo obtusirostris & Poissons & EN & NA & NA \\
\hline Balkans & Matica river and Bacina lakes & Scardinius plotizza & Poissons & NA & oui & NA \\
\hline Balkans & Matica river and Bacina lakes & Squalius microlepis & Poissons & EN & oui & NA \\
\hline Balkans & Matica river and Bacina lakes & Antibaria notata & Mollusques & NA & oui & NA \\
\hline Balkans & Matica river and Bacina lakes & Belgrandia torifera & Mollusques & VU & oui & NA \\
\hline Balkans & Matica river and Bacina lakes & Emmericia expansilabris & Mollusques & VU & oui & NA \\
\hline Balkans & Matica river and Bacina lakes & Emmericia ventricosa & Mollusques & VU & oui & NA \\
\hline Balkans & Matica river and Bacina lakes & Iglica absoloni & Mollusques & NA & oui & NA \\
\hline Balkans & Matica river and Bacina lakes & Iglica bagliviaeformis & Mollusques & EN & NA & NA \\
\hline Balkans & Matica river and Bacina lakes & Paladilhiopsis pretneri & Mollusques & NA & oui & NA \\
\hline Balkans & Matica river and Bacina lakes & Plagigeyeria gladilini & Mollusques & VU & NA & NA \\
\hline Balkans & Matica river and Bacina lakes & Saxurinator brandti & Mollusques & VU & NA & NA \\
\hline
\end{tabular}


Tableau A : Espèces de déclenchement validées dans les Balkans (suite).

\begin{tabular}{|c|c|c|c|c|c|c|}
\hline Sous-région & Nom de la ZCB ${ }^{1}$ & Espèces de déclenchement & Groupe & C1 & $\mathrm{C2}$ & C3 \\
\hline Balkans & Matica river and Bacina lakes & Saxurinator labiatus & Mollusques & $\mathrm{CR}$ & oui & NA \\
\hline Balkans & Matica river and Bacina lakes & Saxurinator montenegrinus & Mollusques & EN & oui & NA \\
\hline Balkans & Matica river and Bacina lakes & Vinodolia fluviatilis & Mollusques & EN & NA & NA \\
\hline Balkans & Mornos & Anguilla anguilla & Poissons & $\mathrm{CR}$ & NA & NA \\
\hline Balkans & Mornos & Economidichthys pygmaeus & Poissons & NA & oui & NA \\
\hline Balkans & Mornos & Pelasgus stymphalicus & Poissons & NA & oui & NA \\
\hline Balkans & Mornos & Valencia letourneuxi & Poissons & CR & oui & NA \\
\hline Balkans & Mornos & Unio crassus & Mollusques & EN & NA & NA \\
\hline Balkans & Naxos & Anguilla anguilla & Poissons & CR & NA & NA \\
\hline Balkans & Neretva delta and associated springs/lakes including Hutovo Blato & Alburnus neretvae & Poissons & NA & oui & NA \\
\hline Balkans & Neretva delta and associated springs/lakes including Hutovo Blato & Cobitis narentana & Poissons & VU & oui & NA \\
\hline Balkans & Neretva delta and associated springs/lakes including Hutovo Blato & Chondrostoma knerii & Poissons & VU & oui & NA \\
\hline Balkans & Neretva delta and associated springs/lakes including Hutovo Blato & Delminichthys adspersus & Poissons & VU & oui & NA \\
\hline Balkans & Neretva delta and associated springs/lakes including Hutovo Blato & Delminichthys ghetaldii & Poissons & VU & oui & NA \\
\hline Balkans & Neretva delta and associated springs/lakes including Hutovo Blato & Knipowitschia croatica & Poissons & VU & oui & NA \\
\hline Balkans & Neretva delta and associated springs/lakes including Hutovo Blato & Knipowitschia radovici & Poissons & VU & oui & NA \\
\hline Balkans & Neretva delta and associated springs/lakes including Hutovo Blato & Rutilus basak & Poissons & NA & oui & NA \\
\hline Balkans & Neretva delta and associated springs/lakes including Hutovo Blato & Salmo obtusirostris & Poissons & EN & NA & NA \\
\hline Balkans & Neretva delta and associated springs/lakes including Hutovo Blato & Scardinius plotizza & Poissons & NA & oui & NA \\
\hline Balkans & Neretva delta and associated springs/lakes including Hutovo Blato & Squalius svallize & Poissons & CR & oui & NA \\
\hline Balkans & Neretva delta and associated springs/lakes including Hutovo Blato & Bithynia mostarensis & Mollusques & NA & oui & NA \\
\hline Balkans & Neretva delta and associated springs/lakes including Hutovo Blato & Congeria kusceri & Mollusques & VU & NA & NA \\
\hline Balkans & Neretva delta and associated springs/lakes including Hutovo Blato & Iglica bagliviaeformis & Mollusques & EN & NA & NA \\
\hline Balkans & Neretva delta and associated springs/lakes including Hutovo Blato & Paladilhiopsis solida & Mollusques & NA & oui & NA \\
\hline Balkans & Neretva delta and associated springs/lakes including Hutovo Blato & Plagigeyeria mostarensis & Mollusques & NA & oui & NA \\
\hline Balkans & Neretva delta and associated springs/lakes including Hutovo Blato & Saxurinator brandti & Mollusques & VU & NA & NA \\
\hline Balkans & Neretva delta and associated springs/lakes including Hutovo Blato & Theodoxus subterrelictus & Mollusques & EN & oui & NA \\
\hline Balkans & Neretva delta and associated springs/lakes including Hutovo Blato & Vinodolia fluviatilis & Mollusques & EN & NA & NA \\
\hline Balkans & $\begin{array}{l}\text { Nevesinjsko polje, Gatacko polje, Cernicko polje, Fatnicko polje and } \\
\text { Dabarsko polje }\end{array}$ & Alburnus neretvae & Poissons & NA & oui & NA \\
\hline Balkans & $\begin{array}{l}\text { Nevesinjsko polje, Gatacko polje, Cernicko polje, Fatnicko polje and } \\
\text { Dabarsko polje }\end{array}$ & Cobitis narentana & Poissons & VU & oui & NA \\
\hline Balkans & $\begin{array}{l}\text { Nevesinjsko polje, Gatacko polje, Cernicko polje, Fatnicko polje and } \\
\text { Dabarsko polje }\end{array}$ & Chondrostoma knerii & Poissons & VU & oui & NA \\
\hline Balkans & $\begin{array}{l}\text { Nevesinjsko polje, Gatacko polje, Cernicko polje, Fatnicko polje and } \\
\text { Dabarsko polje }\end{array}$ & Delminichthys adspersus & Poissons & VU & oui & NA \\
\hline Balkans & $\begin{array}{l}\text { Nevesinjsko polje, Gatacko polje, Cernicko polje, Fatnicko polje and } \\
\text { Dabarsko polje }\end{array}$ & Delminichthys ghetaldii & Poissons & VU & oui & NA \\
\hline Balkans & $\begin{array}{l}\text { Nevesinjsko polje, Gatacko polje, Cernicko polje, Fatnicko polje and } \\
\text { Dabarsko polje }\end{array}$ & Knipowitschia croatica & Poissons & VU & oui & NA \\
\hline Balkans & $\begin{array}{l}\text { Nevesinjsko polje, Gatacko polje, Cernicko polje, Fatnicko polje and } \\
\text { Dabarsko polje }\end{array}$ & Rutilus basak & Poissons & NA & oui & NA \\
\hline Balkans & $\begin{array}{l}\text { Nevesinjsko polje, Gatacko polje, Cernicko polje, Fatnicko polje and } \\
\text { Dabarsko polje }\end{array}$ & Salmo obtusirostris & Poissons & EN & NA & NA \\
\hline Balkans & $\begin{array}{l}\text { Nevesinjsko polje, Gatacko polje, Cernicko polje, Fatnicko polje } \\
\text { and Dabarsko polje }\end{array}$ & Scardinius plotizza & Poissons & NA & oui & NA \\
\hline Balkans & $\begin{array}{l}\text { Nevesinjsko polje, Gatacko polje, Cernicko polje, Fatnicko polje } \\
\text { and Dabarsko polje }\end{array}$ & Squalius svallize & Poissons & $\mathrm{CR}$ & oui & NA \\
\hline Balkans & $\begin{array}{l}\text { Nevesinjsko polje, Gatacko polje, Cernicko polje, Fatnicko polje } \\
\text { and Dabarsko polje }\end{array}$ & Telestes metohiensis & Poissons & VU & oui & NA \\
\hline Balkans & $\begin{array}{l}\text { Nevesinjsko polje, Gatacko polje, Cernicko polje, Fatnicko polje } \\
\text { and Dabarsko polje }\end{array}$ & Bithynia mostarensis & Mollusques & NA & oui & NA \\
\hline
\end{tabular}


Tableau A : Espèces de déclenchement validées dans les Balkans (suite).

\begin{tabular}{|c|c|c|c|c|c|c|}
\hline Sous-région & Nom de la ZCB ${ }^{1}$ & Espèces de déclenchement & Groupe & C1 & $\mathrm{C2}$ & C3 \\
\hline Balkans & $\begin{array}{l}\text { Nevesinjsko polje, Gatacko polje, Cernicko polje, Fatnicko polje } \\
\text { and Dabarsko polje }\end{array}$ & Congeria kusceri & Mollusques & VU & NA & NA \\
\hline Balkans & $\begin{array}{l}\text { Nevesinjsko polje, Gatacko polje, Cernicko polje, Fatnicko polje } \\
\text { and Dabarsko polje }\end{array}$ & Iglica bagliviaeformis & Mollusques & EN & NA & NA \\
\hline Balkans & $\begin{array}{l}\text { Nevesinjsko polje, Gatacko polje, Cernicko polje, Fatnicko polje } \\
\text { and Dabarsko polje }\end{array}$ & Paladilhiopsis solida & Mollusques & NA & oui & NA \\
\hline Balkans & $\begin{array}{l}\text { Nevesinjsko polje, Gatacko polje, Cernicko polje, Fatnicko polje } \\
\text { and Dabarsko polje }\end{array}$ & Plagigeyeria mostarensis & Mollusques & NA & oui & NA \\
\hline Balkans & $\begin{array}{l}\text { Nevesinjsko polje, Gatacko polje, Cernicko polje, Fatnicko polje } \\
\text { and Dabarsko polje }\end{array}$ & Saxurinator brandti & Mollusques & VU & NA & NA \\
\hline Balkans & $\begin{array}{l}\text { Nevesinjsko polje, Gatacko polje, Cernicko polje, Fatnicko polje } \\
\text { and Dabarsko polje }\end{array}$ & Theodoxus subterrelictus & Mollusques & EN & oui & NA \\
\hline Balkans & $\begin{array}{l}\text { Nevesinjsko polje, Gatacko polje, Cernicko polje, Fatnicko polje } \\
\text { and Dabarsko polje }\end{array}$ & Vinodolia fluviatilis & Mollusques & EN & NA & NA \\
\hline Balkans & Northern Korinthiakos & Anguilla anguilla & Poissons & CR & NA & NA \\
\hline Balkans & Pamvotis Lake* & Economidichthys pygmaeus & Poissons & NA & oui & NA \\
\hline Balkans & Pamvotis Lake* & Pelasgus epiroticus & Poissons & CR & oui & NA \\
\hline Balkans & Pamvotis Lake* & Squalius pamvoticus & Poissons & NA & oui & NA \\
\hline Balkans & Pamvotis Lake* & Belgrandiella haesitans & Mollusques & NA & oui & NA \\
\hline Balkans & Pamvotis Lake* & Bithynia graeca & Mollusques & VU & oui & NA \\
\hline Balkans & Pamvotis Lake* & Dreissena blanci & Mollusques & VU & oui & NA \\
\hline Balkans & Pamvotis Lake* & Dreissena presbensis & Mollusques & NA & oui & NA \\
\hline Balkans & Pamvotis Lake* & Islamia epirana & Mollusques & VU & oui & NA \\
\hline Balkans & Pamvotis Lake* & Pseudobithynia westerlundii & Mollusques & NA & oui & NA \\
\hline Balkans & Pamvotis Lake* & Trichonia trichonica & Mollusques & CR & oui & NA \\
\hline Balkans & Part of the Neretva upper catchment & Alburnus neretvae & Poissons & NA & oui & NA \\
\hline Balkans & Part of the Neretva upper catchment & Aulopyge huegelii & Poissons & EN & oui & NA \\
\hline Balkans & Part of the Neretva upper catchment & Cobitis narentana & Poissons & VU & oui & NA \\
\hline Balkans & Part of the Neretva upper catchment & Chondrostoma knerii & Poissons & VU & oui & NA \\
\hline Balkans & Part of the Neretva upper catchment & Delminichthys adspersus & Poissons & VU & oui & NA \\
\hline Balkans & Part of the Neretva upper catchment & Delminichthys ghetaldii & Poissons & VU & oui & NA \\
\hline Balkans & Part of the Neretva upper catchment & Phoxinellus alepidotus & Poissons & EN & oui & NA \\
\hline Balkans & Part of the Neretva upper catchment & Phoxinellus pseudalepidotus & Poissons & VU & oui & NA \\
\hline Balkans & Part of the Neretva upper catchment & Rutilus basak & Poissons & NA & oui & NA \\
\hline Balkans & Part of the Neretva upper catchment & Salmo obtusirostris & Poissons & EN & NA & NA \\
\hline Balkans & Part of the Neretva upper catchment & Scardinius plotizza & Poissons & NA & oui & NA \\
\hline Balkans & Part of the Neretva upper catchment & Squalius svallize & Poissons & CR & oui & NA \\
\hline Balkans & Part of the Neretva upper catchment & Squalius tenellus & Poissons & EN & oui & NA \\
\hline Balkans & Part of the Neretva upper catchment & Bithynia mostarensis & Mollusques & NA & oui & NA \\
\hline Balkans & Part of the Neretva upper catchment & Congeria kusceri & Mollusques & VU & NA & NA \\
\hline Balkans & Part of the Neretva upper catchment & Iglica bagliviaeformis & Mollusques & EN & NA & NA \\
\hline Balkans & Part of the Neretva upper catchment & Paladilhiopsis solida & Mollusques & NA & oui & NA \\
\hline Balkans & Part of the Neretva upper catchment & Plagigeyeria mostarensis & Mollusques & NA & oui & NA \\
\hline Balkans & Part of the Neretva upper catchment & Saxurinator brandti & Mollusques & VU & NA & NA \\
\hline Balkans & Part of the Neretva upper catchment & Theodoxus subterrelictus & Mollusques & EN & oui & NA \\
\hline Balkans & Part of the Neretva upper catchment & Vinodolia fluviatilis & Mollusques & EN & NA & NA \\
\hline Balkans & Part of the Neretva upper catchment - eastern mid catchment & Alburnus neretvae & Poissons & NA & oui & NA \\
\hline Balkans & Part of the Neretva upper catchment - eastern mid catchment & Cobitis narentana & Poissons & VU & oui & NA \\
\hline Balkans & Part of the Neretva upper catchment - eastern mid catchment & Chondrostoma knerii & Poissons & VU & oui & NA \\
\hline Balkans & Part of the Neretva upper catchment - eastern mid catchment & Delminichthys adspersus & Poissons & VU & oui & NA \\
\hline Balkans & Part of the Neretva upper catchment - eastern mid catchment & Delminichthys ghetaldii & Poissons & VU & oui & NA \\
\hline
\end{tabular}


Tableau A : Espèces de déclenchement validées dans les Balkans (suite).

\begin{tabular}{|c|c|c|c|c|c|c|}
\hline Sous-région & Nom de la ZCB ${ }^{1}$ & Espèces de déclenchement & Groupe & C1 & $\mathbf{C 2}$ & C3 \\
\hline Balkans & Part of the Neretva upper catchment - eastern mid catchment & Rutilus basak & Poissons & NA & oui & NA \\
\hline Balkans & Part of the Neretva upper catchment - eastern mid catchment & Salmo obtusirostris & Poissons & EN & NA & NA \\
\hline Balkans & Part of the Neretva upper catchment - eastern mid catchment & Scardinius plotizza & Poissons & NA & oui & NA \\
\hline Balkans & Part of the Neretva upper catchment - eastern mid catchment & Squalius svallize & Poissons & CR & oui & NA \\
\hline Balkans & Part of the Neretva upper catchment - eastern mid catchment & Telestes metohiensis & Poissons & VU & oui & NA \\
\hline Balkans & Part of the Neretva upper catchment - eastern mid catchment & Bithynia mostarensis & Mollusques & NA & oui & NA \\
\hline Balkans & Part of the Neretva upper catchment - eastern mid catchment & Congeria kusceri & Mollusques & VU & NA & NA \\
\hline Balkans & Part of the Neretva upper catchment - eastern mid catchment & Iglica bagliviaeformis & Mollusques & EN & NA & NA \\
\hline Balkans & Part of the Neretva upper catchment - eastern mid catchment & Paladilhiopsis solida & Mollusques & NA & oui & NA \\
\hline Balkans & Part of the Neretva upper catchment - eastern mid catchment & Plagigeyeria mostarensis & Mollusques & NA & oui & NA \\
\hline Balkans & Part of the Neretva upper catchment - eastern mid catchment & Saxurinator brandti & Mollusques & VU & NA & NA \\
\hline Balkans & Part of the Neretva upper catchment - eastern mid catchment & Theodoxus subterrelictus & Mollusques & EN & oui & NA \\
\hline Balkans & Part of the Neretva upper catchment - eastern mid catchment & Vinodolia fluviatilis & Mollusques & EN & NA & NA \\
\hline Balkans & Peloponnese Maleas* & Pseudamnicola exilis & Mollusques & NA & oui & NA \\
\hline Balkans & Pineios Peloponnisou & Pelasgus stymphalicus & Poissons & NA & oui & NA \\
\hline Balkans & Pineios Peloponnisou & Squalius peloponnensis & Poissons & NA & oui & NA \\
\hline Balkans & Pineios Peloponnisou & Tropidophoxinellus hellenicus & Poissons & NA & oui & NA \\
\hline Balkans & Pineios Peloponnisou & Valencia letourneuxi & Poissons & CR & oui & NA \\
\hline Balkans & Pinios Thessalias & Barbus sperchiensis & Poissons & NA & oui & NA \\
\hline Balkans & Pinios Thessalias & Gobio feraeensis & Poissons & VU & oui & NA \\
\hline Balkans & Pinios Thessalias & Paladilhiopsis thessalica & Mollusques & VU & oui & NA \\
\hline Balkans & Popovo polje and Trebisnjica & Cobitis narentana & Poissons & VU & oui & NA \\
\hline Balkans & Popovo polje and Trebisnjica & Delminichthys ghetaldii & Poissons & VU & oui & NA \\
\hline Balkans & Popovo polje and Trebisnjica & Squalius svallize & Poissons & CR & oui & NA \\
\hline Balkans & Popovo polje and Trebisnjica & Antibaria notata & Mollusques & NA & oui & NA \\
\hline Balkans & Popovo polje and Trebisnjica & Belgrandia torifera & Mollusques & VU & oui & NA \\
\hline Balkans & Popovo polje and Trebisnjica & Congeria kusceri & Mollusques & VU & NA & NA \\
\hline Balkans & Popovo polje and Trebisnjica & Emmericia expansilabris & Mollusques & VU & oui & NA \\
\hline Balkans & Popovo polje and Trebisnjica & Emmericia ventricosa & Mollusques & VU & oui & NA \\
\hline Balkans & Popovo polje and Trebisnjica & Iglica absoloni & Mollusques & NA & oui & NA \\
\hline Balkans & Popovo polje and Trebisnjica & Iglica bagliviaeformis & Mollusques & EN & NA & NA \\
\hline Balkans & Popovo polje and Trebisnjica & Lanzaia vjetrenicae & Mollusques & VU & oui & NA \\
\hline Balkans & Popovo polje and Trebisnjica & Narentiana vjetrenicae & Mollusques & EN & oui & NA \\
\hline Balkans & Popovo polje and Trebisnjica & Paladilhiopsis pretneri & Mollusques & NA & oui & NA \\
\hline Balkans & Popovo polje and Trebisnjica & Paladilhiopsis solida & Mollusques & NA & oui & NA \\
\hline Balkans & Popovo polje and Trebisnjica & Plagigeyeria gladilini & Mollusques & VU & NA & NA \\
\hline Balkans & Popovo polje and Trebisnjica & Plagigeyeria tribunicae & Mollusques & VU & oui & NA \\
\hline Balkans & Popovo polje and Trebisnjica & Saxurinator brandti & Mollusques & VU & NA & NA \\
\hline Balkans & Popovo polje and Trebisnjica & Saxurinator labiatus & Mollusques & CR & oui & NA \\
\hline Balkans & Popovo polje and Trebisnjica & Saxurinator montenegrinus & Mollusques & EN & oui & NA \\
\hline Balkans & Popovo polje and Trebisnjica & Vinodolia fluviatilis & Mollusques & EN & NA & NA \\
\hline Balkans & Popovo polje and Trebisnjica & Vinodolia hadouphylax & Mollusques & CR & oui & NA \\
\hline Balkans & Rhodes Island & Ladigesocypris ghigii & Poissons & VU & oui & NA \\
\hline Balkans & Spercheios* & Barbus sperchiensis & Poissons & NA & oui & NA \\
\hline Balkans & Spercheios* & Luciobarbus graecus & Poissons & EN & oui & NA \\
\hline Balkans & Spercheios* & Pelasgus marathonicus & Poissons & NA & oui & NA \\
\hline Balkans & Spercheios* & Pungitius hellenicus & Poissons & CR & oui & NA \\
\hline
\end{tabular}


Tableau A : Espèces de déclenchement validées dans les Balkans (suite).

\begin{tabular}{|c|c|c|c|c|c|c|}
\hline Sous-région & Nom de la ZCB ${ }^{1}$ & Espèces de déclenchement & Groupe & C1 & C2 & C3 \\
\hline Balkans & Tempi & Barbus sperchiensis & Poissons & NA & oui & NA \\
\hline Balkans & Tempi & Gobio feraeensis & Poissons & VU & oui & NA \\
\hline Balkans & Tempi & Knipowitschia thessala & Poissons & EN & oui & NA \\
\hline Balkans & Tempi & Daphniola exigua & Mollusques & EN & oui & NA \\
\hline Balkans & Tempi & Paladilhiopsis thessalica & Mollusques & VU & oui & NA \\
\hline Balkans & Thassos & Turcorientalia hohenackeri & Mollusques & VU & oui & NA \\
\hline Balkans & Thassos & Ceriagrion georgifreyi & Odonates & VU & NA & NA \\
\hline Balkans & Tragos & Pelasgus stymphalicus & Poissons & NA & oui & NA \\
\hline Balkans & Tragos & Squalius peloponnensis & Poissons & NA & oui & NA \\
\hline Balkans & Tragos & Hauffenia edlingeri & Mollusques & CR & oui & NA \\
\hline Balkans & Tragos & Pyrrhosoma elisabethae & Odonates & $\mathrm{CR}$ & oui & NA \\
\hline Balkans & Transboundary Prespa Park* & Alburnoides prespensis & Poissons & VU & oui & oui \\
\hline Balkans & Transboundary Prespa Park* & Alburnus belvica & Poissons & VU & oui & oui \\
\hline Balkans & Transboundary Prespa Park* & Cobitis meridionalis & Poissons & VU & oui & oui \\
\hline Balkans & Transboundary Prespa Park* & Chondrostoma prespense & Poissons & VU & oui & NA \\
\hline Balkans & Transboundary Prespa Park* & Pelasgus prespensis & Poissons & EN & oui & oui \\
\hline Balkans & Transboundary Prespa Park* & Rutilus prespensis & Poissons & VU & oui & oui \\
\hline Balkans & Transboundary Prespa Park* & Salmo peristericus & Poissons & EN & oui & NA \\
\hline Balkans & Transboundary Prespa Park* & Squalius prespensis & Poissons & NA & oui & oui \\
\hline Balkans & Transboundary Prespa Park* & Bithynia prespensis & Mollusques & EN & oui & NA \\
\hline Balkans & Transboundary Prespa Park* & Dreissena blanci & Mollusques & VU & oui & NA \\
\hline Balkans & Transboundary Prespa Park* & Gyraulus stankovici & Mollusques & EN & oui & NA \\
\hline Balkans & Transboundary Prespa Park* & Malaprespia albanica & Mollusques & CR & oui & NA \\
\hline Balkans & Transboundary Prespa Park* & Parabythinella macedonica & Mollusques & EN & oui & NA \\
\hline Balkans & Transboundary Prespa Park* & Parabythinella malaprespensis & Mollusques & CR & oui & NA \\
\hline Balkans & Transboundary Prespa Park* & Pisidium maasseni & Mollusques & EN & oui & NA \\
\hline Balkans & Transboundary Prespa Park* & Planorbis presbensis & Mollusques & VU & oui & NA \\
\hline Balkans & Transboundary Prespa Park* & Prespolitorea malaprespensis & Mollusques & CR & oui & NA \\
\hline Balkans & Transboundary Prespa Park* & Prespolitorea valvataeformis & Mollusques & CR & oui & NA \\
\hline Balkans & Transboundary Prespa Park* & Pyrgohydrobia prespaensis & Mollusques & EN & oui & NA \\
\hline Balkans & Transboundary Prespa Park* & Radix pinteri & Mollusques & EN & oui & NA \\
\hline Balkans & Transboundary Prespa Park* & Vinodolia lacustris & Mollusques & CR & oui & NA \\
\hline Balkans & Trebizat drainage including Imotsko polje* & Alburnus neretvae & Poissons & NA & oui & NA \\
\hline Balkans & Trebizat drainage including Imotsko polje* & Cobitis illyrica & Poissons & CR & oui & NA \\
\hline Balkans & Trebizat drainage including Imotsko polje* & Cobitis narentana & Poissons & VU & oui & NA \\
\hline Balkans & Trebizat drainage including Imotsko polje* & Chondrostoma knerii & Poissons & VU & oui & NA \\
\hline Balkans & Trebizat drainage including Imotsko polje* & Delminichthys adspersus & Poissons & VU & oui & NA \\
\hline Balkans & Trebizat drainage including Imotsko polje* & Delminichthys ghetaldii & Poissons & VU & oui & NA \\
\hline Balkans & Trebizat drainage including Imotsko polje* & Knipowitschia croatica & Poissons & VU & oui & NA \\
\hline Balkans & Trebizat drainage including Imotsko polje* & Rutilus basak & Poissons & NA & oui & NA \\
\hline Balkans & Trebizat drainage including Imotsko polje* & Salmo obtusirostris & Poissons & EN & NA & NA \\
\hline Balkans & Trebizat drainage including Imotsko polje* & Scardinius plotizza & Poissons & NA & oui & NA \\
\hline Balkans & Trebizat drainage including Imotsko polje* & Squalius microlepis & Poissons & EN & oui & NA \\
\hline Balkans & Trebizat drainage including Imotsko polje* & Squalius svallize & Poissons & CR & oui & NA \\
\hline Balkans & Trebizat drainage including Imotsko polje* & Bithynia mostarensis & Mollusques & NA & oui & NA \\
\hline Balkans & Trebizat drainage including Imotsko polje* & Congeria kusceri & Mollusques & VU & NA & NA \\
\hline Balkans & Trebizat drainage including Imotsko polje* & Iglica bagliviaeformis & Mollusques & EN & NA & NA \\
\hline
\end{tabular}


Tableau A : Espèces de déclenchement validées dans les Balkans (suite).

\begin{tabular}{|c|c|c|c|c|c|c|}
\hline Sous-région & Nom de la ZCB ${ }^{1}$ & Espèces de déclenchement & Groupe & C1 & $\mathbf{C 2}$ & C3 \\
\hline Balkans & Trebizat drainage including Imotsko polje* & Paladilhiopsis solida & Mollusques & NA & oui & NA \\
\hline Balkans & Trebizat drainage including Imotsko polje* & Plagigeyeria mostarensis & Mollusques & NA & oui & NA \\
\hline Balkans & Trebizat drainage including Imotsko polje* & Saxurinator brandti & Mollusques & VU & NA & NA \\
\hline Balkans & Trebizat drainage including Imotsko polje* & Theodoxus subterrelictus & Mollusques & EN & oui & NA \\
\hline Balkans & Trebizat drainage including Imotsko polje* & Vinodolia fluviatilis & Mollusques & EN & NA & NA \\
\hline Balkans & Tributaries of lower and middle Neretva including Hutovo Blato & Alburnus neretvae & Poissons & NA & oui & NA \\
\hline Balkans & Tributaries of lower and middle Neretva including Hutovo Blato & Cobitis narentana & Poissons & VU & oui & NA \\
\hline Balkans & Tributaries of lower and middle Neretva including Hutovo Blato & Chondrostoma knerii & Poissons & VU & oui & NA \\
\hline Balkans & Tributaries of lower and middle Neretva including Hutovo Blato & Delminichthys adspersus & Poissons & VU & oui & NA \\
\hline Balkans & Tributaries of lower and middle Neretva including Hutovo Blato & Delminichthys ghetaldii & Poissons & VU & oui & NA \\
\hline Balkans & Tributaries of lower and middle Neretva including Hutovo Blato & Knipowitschia croatica & Poissons & VU & oui & NA \\
\hline Balkans & Tributaries of lower and middle Neretva including Hutovo Blato & Rutilus basak & Poissons & NA & oui & NA \\
\hline Balkans & Tributaries of lower and middle Neretva including Hutovo Blato & Salmo obtusirostris & Poissons & EN & NA & NA \\
\hline Balkans & Tributaries of lower and middle Neretva including Hutovo Blato & Scardinius plotizza & Poissons & NA & oui & NA \\
\hline Balkans & Tributaries of lower and middle Neretva including Hutovo Blato & Squalius svallize & Poissons & CR & oui & NA \\
\hline Balkans & Tributaries of lower and middle Neretva including Hutovo Blato & Bithynia mostarensis & Mollusques & NA & oui & NA \\
\hline Balkans & Tributaries of lower and middle Neretva including Hutovo Blato & Congeria kusceri & Mollusques & VU & NA & NA \\
\hline Balkans & Tributaries of lower and middle Neretva including Hutovo Blato & Iglica bagliviaeformis & Mollusques & EN & NA & NA \\
\hline Balkans & Tributaries of lower and middle Neretva including Hutovo Blato & Paladilhiopsis solida & Mollusques & NA & oui & NA \\
\hline Balkans & Tributaries of lower and middle Neretva including Hutovo Blato & Plagigeyeria mostarensis & Mollusques & NA & oui & NA \\
\hline Balkans & Tributaries of lower and middle Neretva including Hutovo Blato & Saxurinator brandti & Mollusques & VU & NA & NA \\
\hline Balkans & Tributaries of lower and middle Neretva including Hutovo Blato & Theodoxus subterrelictus & Mollusques & EN & oui & NA \\
\hline Balkans & Tributaries of lower and middle Neretva including Hutovo Blato & Vinodolia fluviatilis & Mollusques & EN & NA & NA \\
\hline Balkans & Upper Alfeios & Pelasgus laconicus & Poissons & CR & oui & NA \\
\hline Balkans & Upper Alfeios & Pelasgus stymphalicus & Poissons & NA & oui & NA \\
\hline Balkans & Upper Alfeios & Squalius peloponnensis & Poissons & NA & oui & NA \\
\hline Balkans & Upper Alfeios & Pseudamnicola exilis & Mollusques & NA & oui & NA \\
\hline Balkans & Upper Alfeios & Pyrrhosoma elisabethae & Odonates & $\mathrm{CR}$ & oui & NA \\
\hline Balkans & Upper Aliakmon & Salmo pelagonicus & Poissons & VU & oui & NA \\
\hline Balkans & Upper Aliakmon & Heleobia tritonum & Mollusques & CR & oui & NA \\
\hline Balkans & Upper Aoos & Oxynoemacheilus pindus & Poissons & VU & oui & NA \\
\hline Balkans & Upper Aoos & Squalius sp. nov. 'Aoos' & Poissons & NA & oui & NA \\
\hline Balkans & Upper Aoos & Radomaniola albanica & Mollusques & NA & oui & NA \\
\hline Balkans & Upper Kifissos* & Daphniola louisi & Mollusques & CR & oui & NA \\
\hline Balkans & Vistonis & Alburnus vistonicus & Poissons & $\mathrm{CR}$ & oui & NA \\
\hline Balkans & Vistonis & Alosa vistonica & Poissons & CR & oui & NA \\
\hline Balkans & Volvi-Koronia & Alburnus sp. nov. 'Volvi' & Poissons & NA & oui & NA \\
\hline Balkans & Volvi-Koronia & Alburnus volviticus & Poissons & EN & oui & NA \\
\hline Balkans & Volvi-Koronia & Alosa macedonica & Poissons & VU & oui & NA \\
\hline Balkans & West Karst poljes & Aulopyge huegelii & Poissons & EN & oui & NA \\
\hline Balkans & West Karst poljes & Cobitis dalmatina & Poissons & VU & oui & NA \\
\hline Balkans & West Karst poljes & Chondrostoma phoxinus & Poissons & EN & oui & NA \\
\hline Balkans & West Karst poljes & Delminichthys adspersus & Poissons & VU & oui & NA \\
\hline Balkans & West Karst poljes & Delminichthys ghetaldii & Poissons & VU & oui & NA \\
\hline Balkans & West Karst poljes & Phoxinellus alepidotus & Poissons & EN & oui & NA \\
\hline Balkans & West Karst poljes & Rutilus basak & Poissons & NA & oui & NA \\
\hline Balkans & West Karst poljes & Salmo obtusirostris & Poissons & EN & NA & NA \\
\hline
\end{tabular}


Tableau A : Espèces de déclenchement validées dans les Balkans (suite).

\begin{tabular}{|c|c|c|c|c|c|c|}
\hline Sous-région & Nom de la ZCB' & Espèces de déclenchement & Groupe & C1 & $\mathbf{C 2}$ & C3 \\
\hline Balkans & West Karst poljes & Scardinius dergle & Poissons & NA & oui & NA \\
\hline Balkans & West Karst poljes & Scardinius plotizza & Poissons & NA & oui & NA \\
\hline Balkans & West Karst poljes & Squalius illyricus & Poissons & NA & oui & NA \\
\hline Balkans & West Karst poljes & Squalius tenellus & Poissons & EN & oui & NA \\
\hline Balkans & West Karst poljes & Antibaria notata & Mollusques & NA & oui & NA \\
\hline Balkans & West Karst poljes & Belgrandia torifera & Mollusques & VU & oui & NA \\
\hline Balkans & West Karst poljes & Congeria kusceri & Mollusques & VU & NA & NA \\
\hline Balkans & West Karst poljes & Emmericia expansilabris & Mollusques & VU & oui & NA \\
\hline Balkans & West Karst poljes & Emmericia ventricosa & Mollusques & VU & oui & NA \\
\hline Balkans & West Karst poljes & Iglica absoloni & Mollusques & NA & oui & NA \\
\hline Balkans & West Karst poljes & Iglica bagliviaeformis & Mollusques & EN & NA & NA \\
\hline Balkans & West Karst poljes & Lanzaia kotlusae & Mollusques & VU & oui & NA \\
\hline Balkans & West Karst poljes & Paladilhiopsis pretneri & Mollusques & NA & oui & NA \\
\hline Balkans & West Karst poljes & Plagigeyeria gladilini & Mollusques & VU & NA & NA \\
\hline Balkans & West Karst poljes & Saxurinator brandti & Mollusques & VU & NA & NA \\
\hline Balkans & West Karst poljes & Saxurinator labiatus & Mollusques & $\mathrm{CR}$ & oui & NA \\
\hline Balkans & West Karst poljes & Saxurinator montenegrinus & Mollusques & EN & oui & NA \\
\hline Balkans & West Karst poljes & Vinodolia fluviatilis & Mollusques & EN & NA & NA \\
\hline Balkans & West Karst poljes & Damasonium polyspermum & Plantes & VU & NA & NA \\
\hline Balkans & Yliki-Paralimni-Kifissos* & Luciobarbus graecus & Poissons & EN & oui & NA \\
\hline Balkans & Yliki-Paralimni-Kifissos* & Pelasgus marathonicus & Poissons & NA & oui & NA \\
\hline Balkans & Yliki-Paralimni-Kifissos* & Rutilus ylikiensis & Poissons & EN & oui & NA \\
\hline Balkans & Yliki-Paralimni-Kifissos* & Scardinius graecus & Poissons & $\mathrm{CR}$ & oui & NA \\
\hline Balkans & Yliki-Paralimni-Kifissos* & Telestes beoticus & Poissons & EN & oui & NA \\
\hline Balkans & Zakynthos & Fissuria raehlei & Mollusques & NA & oui & NA \\
\hline Balkans & Zakynthos & Pseudamnicola macrostoma & Mollusques & NA & oui & NA \\
\hline Balkans & Zakynthos & Ceriagrion georgifreyi & Odonates & VU & NA & NA \\
\hline Balkans & Zrmanja River & Aulopyge huegelii & Poissons & EN & oui & NA \\
\hline Balkans & Zrmanja River & Squalius zrmanjae & Poissons & NA & oui & NA \\
\hline Balkans & Zrmanja River & Belgrandia torifera & Mollusques & VU & oui & NA \\
\hline Balkans & Zrmanja River & Belgrandiella krupensis & Mollusques & NA & oui & NA \\
\hline Balkans & Zrmanja River & Belgrandiella zermanica & Mollusques & VU & oui & NA \\
\hline Balkans & Zrmanja River & Congeria kusceri & Mollusques & VU & NA & NA \\
\hline Balkans & Zrmanja River & Hadziella sketi & Mollusques & VU & oui & NA \\
\hline Balkans & Zrmanja River & Saxurinator sketi & Mollusques & EN & oui & NA \\
\hline Balkans & Zrmanja River & Vinodolia fluviatilis & Mollusques & EN & NA & NA \\
\hline Balkans & Zrmanja River & Damasonium polyspermum & Plantes & VU & NA & NA \\
\hline
\end{tabular}

1 N.D.T. : Les noms des sites ZCB figurant dans les tableaux sont en anglais. Toutefois, les noms des sites ZCB cités dans le corps de ce document ont été traduits en français en précisant leur nom anglais entre crochets (« [nom anglais : $\mathrm{XXX]}$ ), afin de permettre au lecteur de faire le lien avec les noms des sites en anglais figurant dans les tableaux correspondants et dans la base de données en ligne (WBDB, World Biodiversity DataBase). 
Tableau B : Espèces de déclenchement validées en Afrique du Nord. C1 (Espèces menacées : CR En danger critique, EN En danger, VU Vulnérable) ; C2 (Aire de répartition restreinte) ; C3 (Communauté restreinte à un biome). * Sites AZE. NA : non applicable.

\begin{tabular}{|c|c|c|c|c|c|c|}
\hline Sous-région & Nom de la ZCB ${ }^{1}$ & Espèces de déclenchement & Groupe & C1 & $\mathrm{C} 2$ & C3 \\
\hline Afrique du Nord & Abid river downstream & Barbus harterti & Poissons & VU & NA & oui \\
\hline Afrique du Nord & Abid river downstream & Barbus nasus & Poissons & NA & NA & oui \\
\hline Afrique du Nord & Abid river downstream & Barbus paytonii & Poissons & VU & NA & oui \\
\hline Afrique du Nord & Abid river downstream & Giustia costata & Mollusques & $\mathrm{CR}$ & oui & NA \\
\hline Afrique du Nord & Abid river downstream & Heideella sp. nov. 'valai' & Mollusques & $\mathrm{CR}$ & oui & NA \\
\hline Afrique du Nord & Abid river downstream & Margaritifera marocana & Mollusques & CR & NA & NA \\
\hline Afrique du Nord & Abid river downstream & Theodoxus marteli & Mollusques & VU & NA & NA \\
\hline Afrique du Nord & Abid river downstream & Theodoxus numidicus & Mollusques & VU & NA & NA \\
\hline Afrique du Nord & Abid river downstream & Unio durieui & Mollusques & EN & NA & NA \\
\hline Afrique du Nord & Abid river downstream & Calopteryx exul & Odonates & EN & NA & NA \\
\hline Afrique du Nord & Abid river downstream & Cordulegaster princeps & Odonates & NA & oui & NA \\
\hline Afrique du Nord & Abid river downstream & Campanula mairei & Plantes & VU & NA & NA \\
\hline Afrique du Nord & Abid river downstream & Damasonium polyspermum & Plantes & VU & NA & NA \\
\hline Afrique du Nord & Abid river downstream & Euphorbia nereidum & Plantes & VU & oui & NA \\
\hline Afrique du Nord & Arhreme River* & Anguilla anguilla & Poissons & $\mathrm{CR}$ & NA & NA \\
\hline Afrique du Nord & Arhreme River* & Barbus issenensis & Poissons & VU & oui & oui \\
\hline Afrique du Nord & Arhreme River* & Barbus massaensis & Poissons & NA & oui & oui \\
\hline Afrique du Nord & Arhreme River* & Iglica soussensis & Mollusques & CR & oui & oui \\
\hline Afrique du Nord & Arhreme River* & Maroccopsis agadirensis & Mollusques & EN & oui & oui \\
\hline Afrique du Nord & Assif El Mal & Cordulegaster princeps & Odonates & NA & oui & NA \\
\hline Afrique du Nord & Assif El Mal & Carum asinorum & Plantes & EN & oui & NA \\
\hline Afrique du Nord & Assif El Mal & Damasonium polyspermum & Plantes & VU & NA & NA \\
\hline Afrique du Nord & Assif El Mal & Eryngium variifolium & Plantes & VU & oui & NA \\
\hline Afrique du Nord & Assif El Mal & Limonium ornatum & Plantes & VU & oui & NA \\
\hline Afrique du Nord & Assif El Mal east & Cordulegaster princeps & Odonates & NA & oui & NA \\
\hline Afrique du Nord & Assif El Mal east & Carum asinorum & Plantes & EN & oui & NA \\
\hline Afrique du Nord & Assif Meloul river & Barbus nasus & Poissons & NA & NA & oui \\
\hline Afrique du Nord & Assif Meloul river & Barbus paytonii & Poissons & VU & NA & oui \\
\hline Afrique du Nord & Assif Meloul river & Giustia costata & Mollusques & $\mathrm{CR}$ & oui & NA \\
\hline Afrique du Nord & Assif Meloul river & Gyraulus laevis & Mollusques & NA & oui & NA \\
\hline Afrique du Nord & Assif Meloul river & Heideella sp. nov. 'valai' & Mollusques & $\mathrm{CR}$ & oui & NA \\
\hline Afrique du Nord & Assif Meloul river & Theodoxus marteli & Mollusques & VU & NA & NA \\
\hline Afrique du Nord & Assif Meloul river & Theodoxus numidicus & Mollusques & VU & NA & NA \\
\hline Afrique du Nord & Assif Meloul river & Unio durieui & Mollusques & EN & NA & NA \\
\hline Afrique du Nord & Assif Meloul river & Calopteryx exul & Odonates & EN & NA & NA \\
\hline Afrique du Nord & Assif Meloul river & Cordulegaster princeps & Odonates & NA & oui & NA \\
\hline Afrique du Nord & Assif Meloul river & Carum lacuum & Plantes & VU & NA & NA \\
\hline Afrique du Nord & Assif Meloul river & Cirsium ducellieri & Plantes & VU & NA & NA \\
\hline Afrique du Nord & Assif Meloul river & Damasonium polyspermum & Plantes & VU & NA & NA \\
\hline Afrique du Nord & Beni Belaid & Anguilla anguilla & Poissons & $\mathrm{CR}$ & NA & NA \\
\hline Afrique du Nord & Beni Belaid & Barbus setivimensis & Poissons & NA & NA & oui \\
\hline Afrique du Nord & Beni Belaid & Belgrandiella nana & Mollusques & NA & NA & oui \\
\hline Afrique du Nord & Beni Belaid & Belgrandiella seminium & Mollusques & NA & oui & oui \\
\hline Afrique du Nord & Beni Belaid & Hydrobia elachista & Mollusques & NA & NA & oui \\
\hline Afrique du Nord & Beni Belaid & Pseudamnicola constantinae & Mollusques & NA & oui & oui \\
\hline
\end{tabular}


Tableau B : Espèces de déclenchement validées en Afrique du Nord (suite).

\begin{tabular}{|c|c|c|c|c|c|c|}
\hline Sous-région & Nom de la ZCB ${ }^{1}$ & Espèces de déclenchement & Groupe & C1 & $\mathrm{C} 2$ & C3 \\
\hline Afrique du Nord & Beni Belaid & Pseudamnicola luteola & Mollusques & NA & NA & oui \\
\hline Afrique du Nord & Beni Belaid & Damasonium polyspermum & Plantes & VU & NA & NA \\
\hline Afrique du Nord & Beni Belaid & Rumex algeriensis & Plantes & EN & NA & NA \\
\hline Afrique du Nord & $\begin{array}{l}\text { Cap Serrat - Cap Blanc - Parc national } \\
\text { de l'Ichkeul }\end{array}$ & Pleurodeles nebulosus & Amphibiens & VU & NA & NA \\
\hline Afrique du Nord & $\begin{array}{l}\text { Cap Serrat - Cap Blanc - Parc national } \\
\text { de l'Ichkeul }\end{array}$ & Anguilla anguilla & Poissons & CR & NA & NA \\
\hline Afrique du Nord & $\begin{array}{l}\text { Cap Serrat - Cap Blanc - Parc national } \\
\text { de l'Ichkeul }\end{array}$ & Barbus callensis & Poissons & NA & NA & oui \\
\hline Afrique du Nord & $\begin{array}{l}\text { Cap Serrat - Cap Blanc - Parc national } \\
\text { de l'Ichkeul }\end{array}$ & Anodonta lucasi & Mollusques & CR & oui & NA \\
\hline Afrique du Nord & $\begin{array}{l}\text { Cap Serrat - Cap Blanc - Parc national } \\
\text { de l'Ichkeul }\end{array}$ & Gomphus lucasii & Odonates & VU & NA & oui \\
\hline Afrique du Nord & $\begin{array}{l}\text { Cap Serrat - Cap Blanc - Parc national } \\
\text { de l'Ichkeul }\end{array}$ & Bellis prostrata & Plantes & NA & oui & NA \\
\hline Afrique du Nord & $\begin{array}{l}\text { Cap Serrat - Cap Blanc - Parc national } \\
\text { de l'Ichkeul }\end{array}$ & Pilularia minuta & Plantes & EN & NA & NA \\
\hline Afrique du Nord & $\begin{array}{l}\text { Cap Serrat - Cap Blanc - Parc national } \\
\text { de l'Ichkeul }\end{array}$ & Rumex tunetanus & Plantes & CR & oui & NA \\
\hline Afrique du Nord & $\begin{array}{l}\text { Cap Serrat - Cap Blanc - Parc national } \\
\text { de l'Ichkeul }\end{array}$ & Serapias stenopetala & Plantes & CR & oui & NA \\
\hline Afrique du Nord & Eastern Numidia & Anguilla anguilla & Poissons & CR & NA & NA \\
\hline Afrique du Nord & Eastern Numidia & Barbus callensis & Poissons & NA & NA & oui \\
\hline Afrique du Nord & Eastern Numidia & Pseudophoxinus punicus & Poissons & EN & oui & oui \\
\hline Afrique du Nord & Eastern Numidia & Anodonta lucasi & Mollusques & CR & oui & NA \\
\hline Afrique du Nord & Eastern Numidia & Bellis prostrata & Plantes & NA & oui & NA \\
\hline Afrique du Nord & Eastern Numidia & Convolvulus durandoi & Plantes & CR & oui & NA \\
\hline Afrique du Nord & Eastern Numidia & Epilobium numidicum & Plantes & CR & oui & NA \\
\hline Afrique du Nord & Eastern Numidia & Juncus sorrentinii & Plantes & VU & NA & NA \\
\hline Afrique du Nord & Eastern Numidia & Lepidium violaceum & Plantes & VU & NA & NA \\
\hline Afrique du Nord & Eastern Numidia & Rhynchospora modesti-lucennoi & Plantes & EN & NA & NA \\
\hline Afrique du Nord & Eastern Numidia & Rumex algeriensis & Plantes & EN & NA & NA \\
\hline Afrique du Nord & Eastern Numidia & Serapias stenopetala & Plantes & CR & oui & NA \\
\hline Afrique du Nord & $\begin{array}{l}\text { El Kala - Les Tourbières de Dar Fatma } \\
\text { transboundary site }\end{array}$ & Anguilla anguilla & Poissons & CR & NA & NA \\
\hline Afrique du Nord & $\begin{array}{l}\text { El Kala - Les Tourbières de Dar Fatma } \\
\text { transboundary site }\end{array}$ & Barbus callensis & Poissons & NA & NA & oui \\
\hline Afrique du Nord & $\begin{array}{l}\text { El Kala - Les Tourbières de Dar Fatma } \\
\text { transboundary site }\end{array}$ & Pseudophoxinus punicus & Poissons & EN & oui & oui \\
\hline Afrique du Nord & $\begin{array}{l}\text { El Kala - Les Tourbières de Dar Fatma } \\
\text { transboundary site }\end{array}$ & Pseudamnicola meluzzii & Mollusques & VU & oui & NA \\
\hline Afrique du Nord & $\begin{array}{l}\text { El Kala - Les Tourbières de Dar Fatma } \\
\text { transboundary site }\end{array}$ & Calopteryx exul & Odonates & EN & NA & NA \\
\hline Afrique du Nord & $\begin{array}{l}\text { El Kala - Les Tourbières de Dar Fatma } \\
\text { transboundary site }\end{array}$ & Gomphus lucasii & Odonates & VU & oui & NA \\
\hline Afrique du Nord & $\begin{array}{l}\text { El Kala - Les Tourbières de Dar Fatma } \\
\text { transboundary site }\end{array}$ & Bellis prostrata & Plantes & NA & oui & NA \\
\hline Afrique du Nord & $\begin{array}{l}\text { El Kala - Les Tourbières de Dar Fatma } \\
\text { transboundary site }\end{array}$ & Pilularia minuta & Plantes & EN & NA & NA \\
\hline Afrique du Nord & $\begin{array}{l}\text { El Kala - Les Tourbières de Dar Fatma } \\
\text { transboundary site }\end{array}$ & Rhynchospora modesti-lucennoi & Plantes & EN & NA & NA \\
\hline Afrique du Nord & Figuig oasis and Oued Saoura & Aphanius saourensis & Poissons & CR & NA & NA \\
\hline Afrique du Nord & Figuig oasis and Oued Saoura & Barbus figuiguensis & Poissons & NA & oui & NA \\
\hline Afrique du Nord & Hauts Plateaux & Anguilla anguilla & Poissons & CR & NA & NA \\
\hline Afrique du Nord & Hauts Plateaux & Aphanius apodus & Poissons & DD & NA & NA \\
\hline
\end{tabular}


Tableau B : Espèces de déclenchement validées en Afrique du Nord (suite).

\begin{tabular}{|c|c|c|c|c|c|c|}
\hline Sous-région & Nom de la ZCB' & Espèces de déclenchement & Groupe & C1 & $\mathrm{C2}$ & C3 \\
\hline Afrique du Nord & Hauts Plateaux & Damasonium polyspermum & Plantes & VU & NA & NA \\
\hline Afrique du Nord & Le Grand Nador & Anguilla anguilla & Poissons & CR & NA & NA \\
\hline Afrique du Nord & Le Grand Nador & Calopteryx exul & Odonates & EN & NA & NA \\
\hline Afrique du Nord & Le Grand Nador & Damasonium polyspermum & Plantes & VU & NA & NA \\
\hline Afrique du Nord & Le Grand Nador & Limonium duriaei & Plantes & VU & NA & NA \\
\hline Afrique du Nord & Le Grand Nador & Spergularia embergeri & Plantes & VU & oui & NA \\
\hline Afrique du Nord & Lower Moulouya & Anguilla anguilla & Poissons & $\mathrm{CR}$ & NA & NA \\
\hline Afrique du Nord & Lower Moulouya & Spergularia doumerguei & Plantes & VU & NA & NA \\
\hline Afrique du Nord & Lower Moulouya & Spergularia embergeri & Plantes & VU & oui & NA \\
\hline Afrique du Nord & Lower Souss and tributaries & Leptochloa ginae & Plantes & EN & oui & NA \\
\hline Afrique du Nord & Maden River & Pleurodeles nebulosus & Amphibiens & VU & NA & oui \\
\hline Afrique du Nord & Maden River & Anguilla anguilla & Poissons & CR & NA & NA \\
\hline Afrique du Nord & Maden River & Barbus callensis & Poissons & NA & NA & oui \\
\hline Afrique du Nord & Maden River & Anodonta lucasi & Mollusques & CR & oui & NA \\
\hline Afrique du Nord & Maden River & Calopteryx exul & Odonates & EN & oui & oui \\
\hline Afrique du Nord & Maden River & Gomphus lucasii & Odonates & VU & oui & oui \\
\hline Afrique du Nord & Maden River & Bellis prostrata & Plantes & NA & oui & NA \\
\hline Afrique du Nord & Maden River & Convolvulus durandoi & Plantes & CR & oui & NA \\
\hline Afrique du Nord & Maden River & Serapias stenopetala & Plantes & CR & oui & NA \\
\hline Afrique du Nord & M'Goun river basin & Ranunculus dyris & Plantes & NA & oui & NA \\
\hline Afrique du Nord & Middle N'Fiss river & Eryngium variifolium & Plantes & VU & oui & NA \\
\hline Afrique du Nord & Middle Oum Er Rbia - Beni Mellal & Barbus harterti & Poissons & VU & NA & oui \\
\hline Afrique du Nord & Middle Oum Er Rbia - Beni Mellal & Barbus nasus & Poissons & NA & NA & oui \\
\hline Afrique du Nord & Middle Oum Er Rbia - Beni Mellal & Barbus paytonii & Poissons & VU & NA & oui \\
\hline Afrique du Nord & Middle Oum Er Rbia - Beni Mellal & Giustia costata & Mollusques & CR & NA & NA \\
\hline Afrique du Nord & Middle Oum Er Rbia - Beni Mellal & Giustia mellalensis & Mollusques & CR & NA & NA \\
\hline Afrique du Nord & Middle Oum Er Rbia - Beni Mellal & Giustia saidai & Mollusques & CR & NA & NA \\
\hline Afrique du Nord & Middle Oum Er Rbia - Beni Mellal & Heideella sp. nov. 'valai' & Mollusques & CR & NA & NA \\
\hline Afrique du Nord & Middle Oum Er Rbia - Beni Mellal & Margaritifera marocana & Mollusques & CR & oui & NA \\
\hline Afrique du Nord & Middle Oum Er Rbia - Beni Mellal & Melanopsis mourebeyensis & Mollusques & EN & NA & NA \\
\hline Afrique du Nord & Middle Oum Er Rbia - Beni Mellal & Theodoxus numidicus & Mollusques & VU & NA & NA \\
\hline Afrique du Nord & Middle Oum Er Rbia - Beni Mellal & Unio durieui & Mollusques & EN & NA & NA \\
\hline Afrique du Nord & Middle Oum Er Rbia - Beni Mellal & Calopteryx exul & Odonates & EN & NA & NA \\
\hline Afrique du Nord & Middle Oum Er Rbia - Beni Mellal & Cirsium ducellieri & Plantes & VU & NA & NA \\
\hline Afrique du Nord & Middle Oum Er Rbia - Beni Mellal & Euphorbia nereidum & Plantes & VU & NA & NA \\
\hline Afrique du Nord & Middle Upper Moulouya & Heideella andreae & Mollusques & NA & oui & NA \\
\hline Afrique du Nord & Middle Upper Moulouya & Melanopsis scalaris & Mollusques & EN & NA & NA \\
\hline Afrique du Nord & Middle Upper Moulouya & Pseudamnicola leprevieri & Mollusques & $\mathrm{CR}$ & oui & NA \\
\hline Afrique du Nord & Middle Upper Moulouya & Pseudamnicola pallaryi & Mollusques & CR & oui & NA \\
\hline Afrique du Nord & Middle Upper Moulouya & Theodoxus marteli & Mollusques & VU & NA & NA \\
\hline Afrique du Nord & Middle Upper Moulouya & Calopteryx exul & Odonates & EN & NA & NA \\
\hline Afrique du Nord & Middle Upper Moulouya & Cordulegaster princeps & Odonates & NA & oui & NA \\
\hline Afrique du Nord & Middle Upper Moulouya & Cirsium ducellieri & Plantes & VU & NA & NA \\
\hline Afrique du Nord & Oued Amizmiz & Cordulegaster princeps & Odonates & NA & oui & NA \\
\hline Afrique du Nord & Oued Amizmiz & Carum asinorum & Plantes & EN & oui & NA \\
\hline Afrique du Nord & Oued Amizmiz & Damasonium polyspermum & Plantes & VU & NA & NA \\
\hline
\end{tabular}


Tableau B : Espèces de déclenchement validées en Afrique du Nord (suite).

\begin{tabular}{|c|c|c|c|c|c|c|}
\hline Sous-région & Nom de la ZCB ${ }^{1}$ & Espèces de déclenchement & Groupe & C1 & $\mathrm{C} 2$ & C3 \\
\hline Afrique du Nord & Oued Amizmiz & Limonium ornatum & Plantes & VU & oui & NA \\
\hline Afrique du Nord & Oued Bouhlou & Cobitis maroccana & Poissons & VU & NA & NA \\
\hline Afrique du Nord & Oued Bouhlou & Heideella knidirii & Mollusques & EN & oui & NA \\
\hline Afrique du Nord & Oued Bouhlou & Horatia sp. nov. 'haasei' & Mollusques & EN & oui & NA \\
\hline Afrique du Nord & Oued Bouhlou & Calopteryx exul & Odonates & EN & NA & NA \\
\hline Afrique du Nord & Oued Bouhlou & Cordulegaster princeps & Odonates & NA & oui & NA \\
\hline Afrique du Nord & Oued Bouhlou & Plantago lacustris & Plantes & VU & oui & NA \\
\hline Afrique du Nord & Oued Bouregreg & Anguilla anguilla & Poissons & CR & NA & NA \\
\hline Afrique du Nord & Oued Bouregreg & Barbus harterti & Poissons & VU & NA & NA \\
\hline Afrique du Nord & Oued Bouregreg & Melanopsis magnifica & Mollusques & EN & NA & NA \\
\hline Afrique du Nord & Oued Bouregreg & Unio durieui & Mollusques & EN & NA & NA \\
\hline Afrique du Nord & Oued Bouregreg & Calopteryx exul & Odonates & EN & NA & NA \\
\hline Afrique du Nord & Oued Bouregreg & Callitriche mathezii & Plantes & EN & NA & NA \\
\hline Afrique du Nord & Oued Bouregreg & Lotus benoistii & Plantes & CR & oui & NA \\
\hline Afrique du Nord & Oued Bouregreg & Pilularia minuta & Plantes & EN & NA & NA \\
\hline Afrique du Nord & Oued el Harrach & Anguilla anguilla & Poissons & CR & NA & NA \\
\hline Afrique du Nord & Oued el Harrach & Barbus setivimensis & Poissons & NA & NA & oui \\
\hline Afrique du Nord & Oued el Harrach & Belgrandiella nana & Mollusques & NA & NA & oui \\
\hline Afrique du Nord & Oued el Harrach & Hydrobia brondeli & Mollusques & NA & NA & oui \\
\hline Afrique du Nord & Oued el Harrach & Mercuria perforata & Mollusques & NA & NA & oui \\
\hline Afrique du Nord & Oued el Harrach & Sphaerium maroccanum & Mollusques & NA & NA & oui \\
\hline Afrique du Nord & Oued el Harrach & Lepidium violaceum & Plantes & VU & NA & NA \\
\hline Afrique du Nord & Oued el Harrach & Rumex algeriensis & Plantes & EN & NA & NA \\
\hline Afrique du Nord & Oued Imouzzer Kandar & Cobitis maroccana & Poissons & VU & NA & NA \\
\hline Afrique du Nord & Oued Imouzzer Kandar & Horatia sp. nov. 'haasei' & Mollusques & EN & NA & NA \\
\hline Afrique du Nord & Oued Imouzzer Kandar & Melanopsis scalaris & Mollusques & EN & NA & NA \\
\hline Afrique du Nord & Oued Imouzzer Kandar & Theodoxus marteli & Mollusques & VU & NA & NA \\
\hline Afrique du Nord & Oued Imouzzer Kandar & Theodoxus numidicus & Mollusques & VU & NA & NA \\
\hline Afrique du Nord & Oued Imouzzer Kandar & Calopteryx exul & Odonates & EN & NA & NA \\
\hline Afrique du Nord & Oued Imouzzer Kandar & Cordulegaster princeps & Odonates & NA & oui & NA \\
\hline Afrique du Nord & Oued Imouzzer Kandar & Plantago lacustris & Plantes & VU & oui & NA \\
\hline Afrique du Nord & Oued Ksob - Igrounzar & Anguilla anguilla & Poissons & CR & NA & NA \\
\hline Afrique du Nord & Oued Ksob - Igrounzar & Barbus ksibi & Poissons & VU & oui & NA \\
\hline Afrique du Nord & Oued Ksob - Igrounzar & Hydrobia maroccana & Mollusques & EN & NA & NA \\
\hline Afrique du Nord & Oued Ksob - Igrounzar & Melanopsis chlorotica & Mollusques & CR & oui & NA \\
\hline Afrique du Nord & Oued Ksob - Igrounzar & Melanopsis turgida & Mollusques & NA & oui & NA \\
\hline Afrique du Nord & Oued Lakhdar & Barbus harterti & Poissons & VU & NA & oui \\
\hline Afrique du Nord & Oued Lakhdar & Barbus nasus & Poissons & NA & NA & oui \\
\hline Afrique du Nord & Oued Lakhdar & Barbus paytonii & Poissons & VU & NA & oui \\
\hline Afrique du Nord & Oued Lakhdar & Giustia costata & Mollusques & CR & oui & oui \\
\hline Afrique du Nord & Oued Lakhdar & Giustia mellalensis & Mollusques & CR & oui & oui \\
\hline Afrique du Nord & Oued Lakhdar & Giustia saidai & Mollusques & CR & oui & oui \\
\hline Afrique du Nord & Oued Lakhdar & Theodoxus marteli & Mollusques & VU & NA & NA \\
\hline Afrique du Nord & Oued Lakhdar & Unio durieui & Mollusques & EN & NA & NA \\
\hline Afrique du Nord & Oued Lakhdar & Calopteryx exul & Odonates & EN & NA & NA \\
\hline Afrique du Nord & Oued Lakhdar & Cordulegaster princeps & Odonates & NA & oui & NA \\
\hline
\end{tabular}


Tableau B : Espèces de déclenchement validées en Afrique du Nord (suite).

\begin{tabular}{|c|c|c|c|c|c|c|}
\hline Sous-région & Nom de la ZCB' & Espèces de déclenchement & Groupe & C1 & $\mathrm{C} 2$ & C3 \\
\hline Afrique du Nord & Oued Lakhdar & Carex fissirostris & Plantes & EN & NA & NA \\
\hline Afrique du Nord & Oued Lakhdar & Cirsium ducellieri & Plantes & VU & NA & NA \\
\hline Afrique du Nord & Oued Laou & Anguilla anguilla & Poissons & CR & NA & NA \\
\hline Afrique du Nord & Oued Laou & Horatia sp. nov. 'haasei' & Mollusques & EN & oui & NA \\
\hline Afrique du Nord & Oued Laou & Theodoxus marteli & Mollusques & VU & NA & NA \\
\hline Afrique du Nord & Oued Laou & Calopteryx exul & Odonates & EN & NA & NA \\
\hline Afrique du Nord & Oued Laou & Genista ancistrocarpa & Plantes & EN & NA & NA \\
\hline Afrique du Nord & Oued Massa & Anguilla anguilla & Poissons & CR & NA & NA \\
\hline Afrique du Nord & Oued Massa & Barbus massaensis & Poissons & NA & oui & oui \\
\hline Afrique du Nord & Oued Massa & Giustia gofasi & Mollusques & EN & NA & oui \\
\hline Afrique du Nord & Oued Massa & Heideella sp. nov. 'salahi' & Mollusques & EN & oui & oui \\
\hline Afrique du Nord & Oued Massa & Maroccopsis agadirensis & Mollusques & EN & oui & oui \\
\hline Afrique du Nord & Oued Massa & Unio foucauldianus & Mollusques & CR & oui & oui \\
\hline Afrique du Nord & Oued Tizguite and Oued Ouaslane & Cobitis maroccana & Poissons & VU & NA & NA \\
\hline Afrique du Nord & Oued Tizguite and Oued Ouaslane & Giustia midarensis & Mollusques & EN & oui & NA \\
\hline Afrique du Nord & Oued Tizguite and Oued Ouaslane & Heideella knidirii & Mollusques & EN & oui & NA \\
\hline Afrique du Nord & Oued Tizguite and Oued Ouaslane & Horatia sp. nov. 'aghbalensis' & Mollusques & EN & oui & NA \\
\hline Afrique du Nord & Oued Tizguite and Oued Ouaslane & Calopteryx exul & Odonates & EN & NA & NA \\
\hline Afrique du Nord & Oued Zhour & Anguilla anguilla & Poissons & CR & NA & NA \\
\hline Afrique du Nord & Oued Zhour & Barbus callensis & Poissons & NA & oui & oui \\
\hline Afrique du Nord & Oued Zhour & Salmo macrostigma & Poissons & NA & NA & oui \\
\hline Afrique du Nord & Oued Zhour & Rumex algeriensis & Plantes & EN & NA & NA \\
\hline Afrique du Nord & Oued Ziz Errachidia & Barbus lepineyi & Poissons & NA & oui & NA \\
\hline Afrique du Nord & Oued Ziz Errachidia & Melanopsis scalaris & Mollusques & EN & NA & NA \\
\hline Afrique du Nord & Oued Ziz Errachidia & Calopteryx exul & Odonates & EN & NA & NA \\
\hline Afrique du Nord & Saidia Coastal Plain & Anguilla anguilla & Poissons & CR & NA & NA \\
\hline Afrique du Nord & Saidia Coastal Plain & Mercuria perforata & Mollusques & NA & NA & oui \\
\hline Afrique du Nord & Saidia Coastal Plain & Limonium duriaei & Plantes & VU & NA & NA \\
\hline Afrique du Nord & Saidia Coastal Plain & Pulicaria filaginoides & Plantes & CR & NA & NA \\
\hline Afrique du Nord & Saidia Coastal Plain & Spergularia doumerguei & Plantes & VU & NA & NA \\
\hline Afrique du Nord & Saidia Coastal Plain & Spergularia embergeri & Plantes & VU & oui & NA \\
\hline Afrique du Nord & Sehb El Majnoune & Giustia bodoni & Mollusques & EN & oui & oui \\
\hline Afrique du Nord & Sehb El Majnoune & Giustia costata & Mollusques & CR & oui & oui \\
\hline Afrique du Nord & Sehb El Majnoune & Giustia mellalensis & Mollusques & CR & oui & oui \\
\hline Afrique du Nord & Sehb El Majnoune & Giustia saidai & Mollusques & CR & oui & oui \\
\hline Afrique du Nord & Sehb El Majnoune & Cirsium ducellieri & Plantes & VU & NA & NA \\
\hline Afrique du Nord & Sehb El Majnoune & Eryngium variifolium & Plantes & VU & oui & NA \\
\hline Afrique du Nord & Seybouse catchment & Pleurodeles poireti & Amphibiens & EN & NA & NA \\
\hline Afrique du Nord & Seybouse catchment & Anguilla anguilla & Poissons & CR & NA & NA \\
\hline Afrique du Nord & Seybouse catchment & Barbus callensis & Poissons & NA & NA & oui \\
\hline Afrique du Nord & Seybouse catchment & Pseudophoxinus punicus & Poissons & EN & oui & oui \\
\hline Afrique du Nord & Seybouse catchment & Hydrobia elachista & Mollusques & NA & NA & oui \\
\hline Afrique du Nord & Seybouse catchment & Pseudamnicola luteola & Mollusques & NA & NA & oui \\
\hline Afrique du Nord & Seybouse catchment & Calopteryx exul & Odonates & EN & NA & NA \\
\hline Afrique du Nord & Seybouse catchment & Gomphus lucasii & Odonates & VU & oui & NA \\
\hline Afrique du Nord & Seybouse catchment & Damasonium polyspermum & Plantes & VU & NA & NA \\
\hline
\end{tabular}


Tableau B : Espèces de déclenchement validées en Afrique du Nord (suite).

\begin{tabular}{|c|c|c|c|c|c|c|}
\hline Sous-région & Nom de la ZCB ${ }^{1}$ & Espèces de déclenchement & Groupe & C1 & C2 & C3 \\
\hline Afrique du Nord & Seybouse catchment & Rhynchospora modesti-lucennoi & Plantes & EN & NA & NA \\
\hline Afrique du Nord & Seybouse catchment & Rumex algeriensis & Plantes & EN & NA & NA \\
\hline Afrique du Nord & Tafna catchment & Anguilla anguilla & Poissons & CR & NA & NA \\
\hline Afrique du Nord & Tafna catchment & Hydrobia brondeli & Mollusques & NA & NA & oui \\
\hline Afrique du Nord & Tafna catchment & Hydrobia maroccana & Mollusques & EN & NA & NA \\
\hline Afrique du Nord & Tafna catchment & Melanopsis letourneuxi & Mollusques & EN & NA & oui \\
\hline Afrique du Nord & Tafna catchment & Melanopsis scalaris & Mollusques & EN & NA & NA \\
\hline Afrique du Nord & Tafna catchment & Mercuria perforata & Mollusques & NA & NA & oui \\
\hline Afrique du Nord & Tafna catchment & Pseudamnicola luteola & Mollusques & NA & NA & oui \\
\hline Afrique du Nord & Tafna catchment & Sphaerium maroccanum & Mollusques & NA & NA & oui \\
\hline Afrique du Nord & Tafna catchment & Theodoxus marteli & Mollusques & VU & NA & NA \\
\hline Afrique du Nord & Tafna catchment & Damasonium polyspermum & Plantes & VU & NA & NA \\
\hline Afrique du Nord & Tifnout basin & Barbus issenensis & Poissons & VU & oui & oui \\
\hline Afrique du Nord & Tifnout basin & Barbus massaensis & Poissons & NA & oui & oui \\
\hline Afrique du Nord & Tifnout basin & Salmo akairos & Poissons & VU & oui & oui \\
\hline Afrique du Nord & Tifnout basin & Cordulegaster princeps & Odonates & NA & oui & NA \\
\hline Afrique du Nord & Tifnout basin & Carex fissirostris & Plantes & EN & NA & NA \\
\hline Afrique du Nord & Tifnout basin & Romulea antiatlantica & Plantes & CR & oui & NA \\
\hline Afrique du Nord & Tigrigra stream & Cobitis maroccana & Poissons & VU & NA & NA \\
\hline Afrique du Nord & Tigrigra stream & Horatia sp. nov. 'aghbalensis' & Mollusques & EN & oui & NA \\
\hline Afrique du Nord & Tigrigra stream & Melanopsis arbalensis & Mollusques & NA & oui & NA \\
\hline Afrique du Nord & Tigrigra stream & Melanopsis scalaris & Mollusques & EN & NA & NA \\
\hline Afrique du Nord & Tigrigra stream & Theodoxus numidicus & Mollusques & VU & NA & NA \\
\hline Afrique du Nord & Tigrigra stream & Unio durieui & Mollusques & EN & NA & NA \\
\hline Afrique du Nord & Tigrigra stream & Calopteryx exul & Odonates & EN & NA & NA \\
\hline Afrique du Nord & Tigrigra stream & Cordulegaster princeps & Odonates & NA & oui & NA \\
\hline Afrique du Nord & Tigrigra stream & Lepidium violaceum & Plantes & VU & NA & NA \\
\hline Afrique du Nord & Upper Dades & Calopteryx exul & Odonates & EN & NA & NA \\
\hline Afrique du Nord & Upper Dades & Cordulegaster princeps & Odonates & NA & oui & NA \\
\hline Afrique du Nord & Upper Dades & Campanula mairei & Plantes & VU & NA & NA \\
\hline Afrique du Nord & Upper Dades & Ranunculus dyris & Plantes & NA & oui & NA \\
\hline Afrique du Nord & Upper Medjarda River & Anguilla anguilla & Poissons & CR & NA & NA \\
\hline Afrique du Nord & Upper Medjarda River & Barbus callensis & Poissons & NA & NA & oui \\
\hline Afrique du Nord & Upper Medjarda River & Pseudophoxinus punicus & Poissons & EN & NA & NA \\
\hline Afrique du Nord & Upper Medjarda River & Unio durieui & Mollusques & EN & NA & NA \\
\hline Afrique du Nord & Upper Medjarda River & Calopteryx exul & Odonates & EN & NA & NA \\
\hline Afrique du Nord & Upper Medjarda River & Gomphus lucasii & Odonates & VU & oui & NA \\
\hline Afrique du Nord & Upper Oued N'Fiss & Cordulegaster princeps & Odonates & NA & oui & NA \\
\hline Afrique du Nord & Upper Oued N'Fiss & Carum asinorum & Plantes & EN & oui & NA \\
\hline Afrique du Nord & Upper Oum Er Rbia & Barbus harterti & Poissons & VU & NA & oui \\
\hline Afrique du Nord & Upper Oum Er Rbia & Barbus paytonii & Poissons & VU & NA & oui \\
\hline Afrique du Nord & Upper Oum Er Rbia & Melanopsis scalaris & Mollusques & EN & NA & NA \\
\hline Afrique du Nord & Upper Oum Er Rbia & Calopteryx exul & Odonates & EN & NA & NA \\
\hline Afrique du Nord & Upper Oum Er Rbia & Cordulegaster princeps & Odonates & NA & oui & NA \\
\hline Afrique du Nord & Upper Oum Er Rbia & Cirsium ducellieri & Plantes & VU & NA & NA \\
\hline Afrique du Nord & Upper Oum Er Rbia & Damasonium polyspermum & Plantes & VU & NA & NA \\
\hline
\end{tabular}


Tableau B : Espèces de déclenchement validées en Afrique du Nord (suite).

\begin{tabular}{|c|c|c|c|c|c|c|}
\hline Sous-région & Nom de la ZCB ${ }^{1}$ & Espèces de déclenchement & Groupe & C1 & $\mathrm{C} 2$ & C3 \\
\hline Afrique du Nord & Upper Oum Er Rbia & Rorippa hayanica & Plantes & VU & NA & NA \\
\hline Afrique du Nord & Upper Oum Er Rbia above Kasba Tadla & Barbus harterti & Poissons & VU & NA & oui \\
\hline Afrique du Nord & Upper Oum Er Rbia above Kasba Tadla & Barbus nasus & Poissons & NA & NA & oui \\
\hline Afrique du Nord & Upper Oum Er Rbia above Kasba Tadla & Barbus paytonii & Poissons & VU & NA & oui \\
\hline Afrique du Nord & Upper Oum Er Rbia above Kasba Tadla & Gyraulus laevis & Mollusques & NA & oui & NA \\
\hline Afrique du Nord & Upper Oum Er Rbia above Kasba Tadla & Margaritifera marocana & Mollusques & $\mathrm{CR}$ & oui & NA \\
\hline Afrique du Nord & Upper Oum Er Rbia above Kasba Tadla & Theodoxus numidicus & Mollusques & VU & NA & NA \\
\hline Afrique du Nord & Upper Oum Er Rbia above Kasba Tadla & Unio durieui & Mollusques & EN & NA & NA \\
\hline Afrique du Nord & Upper Oum Er Rbia above Kasba Tadla & Calopteryx exul & Odonates & EN & NA & NA \\
\hline Afrique du Nord & Upper Oum Er Rbia above Kasba Tadla & Cirsium ducellieri & Plantes & VU & NA & NA \\
\hline Afrique du Nord & Upper Oum Er Rbia above Kasba Tadla & Damasonium polyspermum & Plantes & VU & NA & NA \\
\hline Afrique du Nord & Western Numidia* & Pleurodeles poireti & Amphibiens & EN & NA & NA \\
\hline Afrique du Nord & Western Numidia* & Anguilla anguilla & Poissons & $\mathrm{CR}$ & NA & NA \\
\hline Afrique du Nord & Western Numidia* & Barbus setivimensis & Poissons & NA & NA & oui \\
\hline Afrique du Nord & Western Numidia* & Pseudophoxinus punicus & Poissons & EN & oui & oui \\
\hline Afrique du Nord & Western Numidia* & Damasonium polyspermum & Plantes & VU & NA & NA \\
\hline Afrique du Nord & Western Numidia* & Rhynchospora modesti-lucennoi & Plantes & EN & NA & NA \\
\hline Afrique du Nord & Western Numidia* & Rumex algeriensis & Plantes & EN & NA & NA \\
\hline
\end{tabular}

1 N.D.T. : Les noms des sites ZCB figurant dans les tableaux sont en anglais. Toutefois, les noms des sites ZCB cités dans le corps de ce document ont été traduits en français en précisant leur nom anglais entre crochets (« [nom anglais : XXX] »), afin de permettre au lecteur de faire le lien avec les noms des sites en anglais figurant dans les tableauX correspondants et dans la base de données en ligne (WBDB, World Biodiversity DataBase). 
Tableau C : Espèces de déclenchement validées en Turquie et au Levant. C1 (Espèces menacées : CR En danger critique, EN En danger, VU Vulnérable) ; C2 (Aire de répartition restreinte); C3 (Communauté restreinte à un biome). * Sites AZE. NA : non applicable.

\begin{tabular}{|c|c|c|c|c|c|c|}
\hline Sous-région & Nom de la ZCB ${ }^{1}$ & Espèces de déclenchement & Groupe & C1 & $\mathrm{C} 2$ & C3 \\
\hline Turquie et Levant & Aksu River & Alburnus baliki & Poissons & EN & oui & NA \\
\hline Turquie et Levant & Aksu River & Capoeta antalyensis & Poissons & VU & oui & NA \\
\hline Turquie et Levant & Aksu River & Oxynoemacheilus mediterraneus & Poissons & NA & oui & NA \\
\hline Turquie et Levant & Aksu River & Pseudophoxinus alii & Poissons & EN & oui & NA \\
\hline Turquie et Levant & Aksu River & Unio crassus & Mollusques & EN & NA & NA \\
\hline Turquie et Levant & Akyaka springs* & Bithynia pesicii & Mollusques & EN & oui & NA \\
\hline Turquie et Levant & Akyaka springs* & Unio crassus & Mollusques & EN & NA & NA \\
\hline Turquie et Levant & Amman & Oxynoemacheilus insignis & Poissons & $\mathrm{NT}^{2}$ & NA & NA \\
\hline Turquie et Levant & Amman & Melanopsis buccinoidea & Mollusques & NA & oui & NA \\
\hline Turquie et Levant & Bakirçay & Alburnus attalus & Poissons & EN & oui & NA \\
\hline Turquie et Levant & Bakirçay & Chondrostoma holmwoodii & Poissons & VU & NA & NA \\
\hline Turquie et Levant & Bakirçay & Knipowitschia mermere & Poissons & VU & oui & NA \\
\hline Turquie et Levant & Bakirçay & Squalius cii & Poissons & NA & oui & NA \\
\hline Turquie et Levant & Bakirçay & Pseudorientalia natolica & Mollusques & $\mathrm{DD}$ & oui & NA \\
\hline Turquie et Levant & Bakirçay & Unio crassus & Mollusques & EN & NA & NA \\
\hline Turquie et Levant & Burdur lake and catchments* & Aphanius sureyanus & Poissons & EN & oui & oui \\
\hline Turquie et Levant & Burdur lake and catchments* & Cobitis phrygica & Poissons & EN & oui & NA \\
\hline Turquie et Levant & Burdur lake and catchments* & Chondrostoma fahirae & Poissons & EN & oui & NA \\
\hline Turquie et Levant & Burdur lake and catchments* & Oxynoemacheilus anatolicus & Poissons & EN & oui & NA \\
\hline Turquie et Levant & Burdur lake and catchments* & Graecoanatolica brevis & Mollusques & $\mathrm{CR}$ & oui & NA \\
\hline Turquie et Levant & Burdur lake and catchments* & Tefennia tefennica & Mollusques & VU & oui & NA \\
\hline Turquie et Levant & Büyük Menderes river & Alburnus demiri & Poissons & VU & oui & NA \\
\hline Turquie et Levant & Büyük Menderes river & Chondrostoma meandrense & Poissons & VU & NA & NA \\
\hline Turquie et Levant & Büyük Menderes river & Luciobarbus kottelati & Poissons & VU & oui & NA \\
\hline Turquie et Levant & Büyük Menderes river & Oxynoemacheilus germencicus & Poissons & VU & NA & NA \\
\hline Turquie et Levant & Büyük Menderes river & Vimba mirabilis & Poissons & NA & oui & NA \\
\hline Turquie et Levant & Büyük Menderes river & Pseudamnicola geldiayana & Mollusques & EN & oui & NA \\
\hline Turquie et Levant & Büyük Menderes river & Unio crassus & Mollusques & EN & NA & NA \\
\hline Turquie et Levant & Central Jordan River & Luciobarbus longiceps & Poissons & EN & NA & NA \\
\hline Turquie et Levant & Central Jordan River & Oxynoemacheilus insignis & Poissons & $\mathrm{NT}^{2}$ & NA & NA \\
\hline Turquie et Levant & Central Jordan River & Melanopsis buccinoidea & Mollusques & NA & oui & NA \\
\hline Turquie et Levant & Central Jordan River & Potomida littoralis & Mollusques & EN & NA & NA \\
\hline Turquie et Levant & Central Jordan River & Unio terminalis & Mollusques & VU & NA & NA \\
\hline Turquie et Levant & Duden river* & Pseudophoxinus antalyae & Poissons & VU & oui & oui \\
\hline Turquie et Levant & Duden river* & Hydrobia anatolica & Mollusques & $\mathrm{CR}$ & oui & NA \\
\hline Turquie et Levant & Eğirdir Lake catchment* & Capoeta pestai & Poissons & $\mathrm{CR}$ & oui & NA \\
\hline Turquie et Levant & Eğirdir Lake catchment* & Pseudophoxinus egridiri & Poissons & EN & oui & NA \\
\hline Turquie et Levant & Eğirdir Lake catchment* & Seminemacheilus ispartensis & Poissons & VU & NA & NA \\
\hline Turquie et Levant & Eğirdir Lake catchment* & Bythinella turca & Mollusques & $\mathrm{CR}$ & oui & NA \\
\hline Turquie et Levant & Eğirdir Lake catchment* & Falsipyrgula pfeiferi & Mollusques & EN & oui & oui \\
\hline Turquie et Levant & Eğirdir Lake catchment* & Graecoanatolica kocapinarica & Mollusques & VU & oui & NA \\
\hline Turquie et Levant & Eğirdir Lake catchment* & Graecoanatolica lacustristurca & Mollusques & EN & oui & oui \\
\hline Turquie et Levant & Gökdere (Yeşildere) stream* & Capoeta mauricii & Poissons & EN & oui & NA \\
\hline Turquie et Levant & Gökdere (Yeşildere) stream & Gobio hettitorum & Poissons & CR & oui & NA \\
\hline Turquie et Levant & Gökdere (Yeşildere) stream* & Oxynoemacheilus eregliensis & Poissons & VU & NA & NA \\
\hline
\end{tabular}


Tableau C : Espèces de déclenchement validées en Turquie et au Levant (suite).

\begin{tabular}{|c|c|c|c|c|c|c|}
\hline Sous-région & Nom de la ZCB ${ }^{1}$ & Espèces de déclenchement & Groupe & C1 & $\mathrm{C}_{2}$ & C3 \\
\hline Turquie et Levant & Gökdere (Yeşildere) stream* & Unio crassus & Mollusques & EN & NA & NA \\
\hline Turquie et Levant & Işıklı/Çivril lake and catchment* & Chondrostoma meandrense & Poissons & VU & NA & NA \\
\hline Turquie et Levant & Işıklı/Çivril lake and catchment* & Gobio maeandricus & Poissons & EN & oui & NA \\
\hline Turquie et Levant & Işıklı/Çivril lake and catchment* & Oxynoemacheilus mesudae & Poissons & EN & oui & NA \\
\hline Turquie et Levant & Işıklı/Çivril lake and catchment* & Pseudophoxinus maeandri & Poissons & EN & oui & NA \\
\hline Turquie et Levant & Işıklı/Çivril lake and catchment* & Pseudophoxinus maeandricus & Poissons & CR & oui & NA \\
\hline Turquie et Levant & Işıklı/Çivril lake and catchment* & Squalius carinus & Poissons & EN & oui & NA \\
\hline Turquie et Levant & Işıklı/Çivril lake and catchment* & Graecoanatolica tenuis & Mollusques & VU & oui & NA \\
\hline Turquie et Levant & Işıklı/Çivril lake and catchment* & Pseudamnicola geldiayana & Mollusques & EN & oui & NA \\
\hline Turquie et Levant & Jerico catchment & Luciobarbus longiceps & Poissons & EN & NA & NA \\
\hline Turquie et Levant & Jerico catchment & Oxynoemacheilus insignis & Poissons & $\mathrm{NT}^{2}$ & NA & NA \\
\hline Turquie et Levant & Jerico catchment & Melanopsis buccinoidea & Mollusques & NA & oui & NA \\
\hline Turquie et Levant & Karpuzçay stream* & Capoeta caelestis & Poissons & NA & oui & NA \\
\hline Turquie et Levant & Karpuzçay stream* & Kirelia murtici & Mollusques & CR & oui & NA \\
\hline Turquie et Levant & Karpuzçay stream* & Unio crassus & Mollusques & EN & NA & NA \\
\hline Turquie et Levant & Köprü Çay* & Alburnus baliki & Poissons & EN & oui & NA \\
\hline Turquie et Levant & Köprü Çay* & Capoeta antalyensis & Poissons & VU & oui & NA \\
\hline Turquie et Levant & Köprü Çay* & Oxynoemacheilus mediterraneus & Poissons & NA & oui & NA \\
\hline Turquie et Levant & Köprü Çay* & Pseudophoxinus fahrettini & Poissons & EN & oui & NA \\
\hline Turquie et Levant & Köprü Çay* & Unio crassus & Mollusques & EN & NA & NA \\
\hline Turquie et Levant & Korkuteli and Elmali plains & Pseudophoxinus evliyae & Poissons & EN & oui & NA \\
\hline Turquie et Levant & Korkuteli and Elmali plains & Graecoanatolica pamphylica & Mollusques & EN & oui & NA \\
\hline Turquie et Levant & Korkuteli and Elmali plains & Unio crassus & Mollusques & EN & NA & NA \\
\hline Turquie et Levant & Korkuteli and Elmali plains & Scardinius elmaliensis & Poissons & EN & NA & NA \\
\hline Turquie et Levant & Küçük Menderes & Oxynoemacheilus germencicus & Poissons & VU & NA & oui \\
\hline Turquie et Levant & Küçük Menderes & Bythinella occasiuncula & Mollusques & VU & oui & NA \\
\hline Turquie et Levant & Küçük Menderes & Pseudorientalia natolica & Mollusques & DD & oui & NA \\
\hline Turquie et Levant & Küçük Menderes & Unio crassus & Mollusques & EN & NA & NA \\
\hline Turquie et Levant & Küçük Menderes & Knipowitschia ephesi & Poissons & CR & oui & oui \\
\hline Turquie et Levant & Lake Beyşehir and catchments* & Capoeta mauricii & Poissons & EN & oui & NA \\
\hline Turquie et Levant & Lake Beyşehir and catchments* & Cobitis battalgili & Poissons & EN & oui & NA \\
\hline Turquie et Levant & Lake Beyşehir and catchments* & Cobitis bilseli & Poissons & EN & oui & NA \\
\hline Turquie et Levant & Lake Beyşehir and catchments* & Chondrostoma beysehirense & Poissons & EN & oui & NA \\
\hline Turquie et Levant & Lake Beyşehir and catchments* & Gobio battalgilae & Poissons & NA & oui & NA \\
\hline Turquie et Levant & Lake Beyşehir and catchments* & Gobio microlepidotus & Poissons & VU & oui & NA \\
\hline Turquie et Levant & Lake Beyşehir and catchments* & Oxynoemacheilus atili & Poissons & NA & oui & NA \\
\hline Turquie et Levant & Lake Beyşehir and catchments* & Pseudophoxinus anatolicus & Poissons & EN & oui & NA \\
\hline Turquie et Levant & Lake Beyşehir and catchments* & Pseudophoxinus hittitorum & Poissons & EN & oui & NA \\
\hline Turquie et Levant & Lake Beyşehir and catchments* & Seminemacheilus lendlii & Poissons & VU & NA & NA \\
\hline Turquie et Levant & Lake Beyşehir and catchments* & Falsipyrgula beysehirana & Mollusques & CR & NA & NA \\
\hline Turquie et Levant & Lake Beyşehir and catchments* & Graecoanatolica lacustristurca & Mollusques & EN & NA & NA \\
\hline Turquie et Levant & Lake Beyşehir and catchments* & Kirelia carinata & Mollusques & CR & oui & NA \\
\hline Turquie et Levant & Lake Homs (Qatinah) & Alburnus orontis & Poissons & VU & oui & NA \\
\hline Turquie et Levant & Lake Homs (Qatinah) & Capoeta barroisi & Poissons & EN & oui & NA \\
\hline Turquie et Levant & Lake Homs (Qatinah) & Anodonta pseudodopsis & Mollusques & EN & oui & NA \\
\hline Turquie et Levant & Lake Homs (Qatinah) & Margaritifera homsensis & Mollusques & EN & oui & NA \\
\hline Turquie et Levant & Lake Homs (Qatinah) & Melanopsis dircaena & Mollusques & EN & oui & NA \\
\hline
\end{tabular}


Tableau C : Espèces de déclenchement validées en Turquie et au Levant (suite).

\begin{tabular}{|c|c|c|c|c|c|c|}
\hline Sous-région & Nom de la ZCB ${ }^{1}$ & Espèces de déclenchement & Groupe & C1 & $\mathbf{C 2}$ & C3 \\
\hline Turquie et Levant & Lake Homs (Qatinah) & Potomida littoralis & Mollusques & EN & NA & NA \\
\hline Turquie et Levant & Lake Homs (Qatinah) & Unio terminalis & Mollusques & VU & NA & NA \\
\hline Turquie et Levant & Lake Iznik and catchment* & Oxynoemacheilus phoxinoides & Poissons & CR & oui & NA \\
\hline Turquie et Levant & Lake Iznik and catchment* & Falsibelgrandiella bunarica & Mollusques & NA & oui & NA \\
\hline Turquie et Levant & Lake Iznik and catchment* & Pseudorientalia natolica & Mollusques & DD & oui & NA \\
\hline Turquie et Levant & Lake Iznik and catchment* & Sadleriana byzanthina & Mollusques & NA & oui & NA \\
\hline Turquie et Levant & Lake Iznik and catchment* & Unio crassus & Mollusques & EN & NA & NA \\
\hline Turquie et Levant & Lakes Acıgöl and Salda & Aphanius transgrediens & Poissons & CR & oui & NA \\
\hline Turquie et Levant & Lakes Acıgöl and Salda & Cobitis phrygica & Poissons & EN & oui & NA \\
\hline Turquie et Levant & Lakes Acıgöl and Salda & Graecoanatolica conica & Mollusques & CR & oui & NA \\
\hline Turquie et Levant & Lakes Acıgöl and Salda & Graecoanatolica tenuis & Mollusques & VU & oui & NA \\
\hline Turquie et Levant & Lakes Acıgöl and Salda & Tefennia tefennica & Mollusques & VU & oui & NA \\
\hline Turquie et Levant & Lakes Akşehir - Eber system & Alburnus nasreddini & Poissons & CR & oui & NA \\
\hline Turquie et Levant & Lakes Akşehir - Eber system & Gobio intermedius & Poissons & EN & oui & NA \\
\hline Turquie et Levant & Lakes Akşehir - Eber system & Seminemacheilus ispartensis & Poissons & VU & oui & NA \\
\hline Turquie et Levant & Lakes Akşehir - Eber system & Squalius recurvirostris & Poissons & VU & oui & NA \\
\hline Turquie et Levant & Lakes Akşehir - Eber system & Bithynia pseudemmericia & Mollusques & VU & oui & NA \\
\hline Turquie et Levant & Litani River* & Oxynoemacheilus leontinae & Poissons & NA & oui & NA \\
\hline Turquie et Levant & Litani River* & Potomida littoralis & Mollusques & EN & NA & NA \\
\hline Turquie et Levant & Lower Asi drainage & Acanthobrama centisquama & Poissons & CR & NA & NA \\
\hline Turquie et Levant & Lower Asi drainage & Alburnus orontis & Poissons & VU & oui & NA \\
\hline Turquie et Levant & Lower Asi drainage & Capoeta barroisi & Poissons & EN & oui & NA \\
\hline Turquie et Levant & Lower Asi drainage & Cobitis levantina & Poissons & EN & oui & NA \\
\hline Turquie et Levant & Lower Asi drainage & Chondrostoma kinzelbachi & Poissons & EN & oui & NA \\
\hline Turquie et Levant & Lower Asi drainage & Oxynoemacheilus hamwii & Poissons & EN & oui & NA \\
\hline Turquie et Levant & Lower Asi drainage & Anodonta pseudodopsis & Mollusques & EN & oui & NA \\
\hline Turquie et Levant & Lower Asi drainage & Potomida littoralis & Mollusques & EN & NA & NA \\
\hline Turquie et Levant & Lower Asi drainage & Unio crassus & Mollusques & EN & NA & NA \\
\hline Turquie et Levant & Lower Asi drainage & Unio terminalis & Mollusques & VU & NA & NA \\
\hline Turquie et Levant & Lower Gediz river & Alburnus battalgilae & Poissons & VU & oui & NA \\
\hline Turquie et Levant & Lower Gediz river & Chondrostoma holmwoodii & Poissons & VU & NA & NA \\
\hline Turquie et Levant & Lower Gediz river & Knipowitschia mermere & Poissons & VU & oui & NA \\
\hline Turquie et Levant & Lower Gediz river & Oxynoemacheilus germencicus & Poissons & VU & NA & NA \\
\hline Turquie et Levant & Lower Gediz river & Vimba mirabilis & Poissons & NA & oui & oui \\
\hline Turquie et Levant & Lower Gediz river & Unio crassus & Mollusques & EN & NA & NA \\
\hline Turquie et Levant & Lower Yarmouk & Luciobarbus longiceps & Poissons & EN & NA & NA \\
\hline Turquie et Levant & Lower Yarmouk & Oxynoemacheilus insignis & Poissons & $\mathrm{NT}^{2}$ & NA & NA \\
\hline Turquie et Levant & Main stem of the Tigris River & Arabibarbus grypus & Poissons & VU & NA & NA \\
\hline Turquie et Levant & Main stem of the Tigris River & Carasobarbus kosswigi & Poissons & VU & NA & NA \\
\hline Turquie et Levant & Main stem of the Tigris River & Luciobarbus esocinus & Poissons & VU & NA & NA \\
\hline Turquie et Levant & Main stem of the Tigris River & Luciobarbus subquincunciatus & Poissons & CR & NA & NA \\
\hline Turquie et Levant & Main stem of the Tigris River & Luciobarbus xanthopterus & Poissons & VU & NA & NA \\
\hline Turquie et Levant & Manavgat River & Alburnus baliki & Poissons & EN & oui & NA \\
\hline Turquie et Levant & Manavgat River & Capoeta caelestis & Poissons & NA & oui & NA \\
\hline Turquie et Levant & Manavgat River & Cobitis battalgili & Poissons & EN & oui & NA \\
\hline Turquie et Levant & Manavgat River & Oxynoemacheilus atili & Poissons & NA & oui & NA \\
\hline
\end{tabular}


Tableau C : Espèces de déclenchement validées en Turquie et au Levant (suite).

\begin{tabular}{|c|c|c|c|c|c|c|}
\hline Sous-région & Nom de la ZCB' & Espèces de déclenchement & Groupe & C1 & $\mathbf{C}_{2}$ & C3 \\
\hline Turquie et Levant & Manavgat River & Unio crassus & Mollusques & EN & NA & NA \\
\hline Turquie et Levant & Middle and lower Seyhan river & Capoeta turani & Poissons & NA & oui & NA \\
\hline Turquie et Levant & Middle and lower Seyhan river & Hemigrammocapoeta culiciphaga & Poissons & NA & oui & NA \\
\hline Turquie et Levant & Middle and lower Seyhan river & Oxynoemacheilus seyhanicola & Poissons & EN & oui & NA \\
\hline Turquie et Levant & Middle and lower Seyhan river & Potomida littoralis & Mollusques & EN & NA & NA \\
\hline Turquie et Levant & Middle and lower Seyhan river & Unio crassus & Mollusques & EN & NA & NA \\
\hline Turquie et Levant & Middle and lower Seyhan river & Unio terminalis & Mollusques & VU & NA & NA \\
\hline Turquie et Levant & Middle Orontes & Alburnus orontis & Poissons & VU & oui & NA \\
\hline Turquie et Levant & Middle Orontes & Melanopsis dircaena & Mollusques & EN & oui & NA \\
\hline Turquie et Levant & Middle Orontes & Potomida littoralis & Mollusques & EN & NA & NA \\
\hline Turquie et Levant & Middle Orontes & Unio terminalis & Mollusques & VU & NA & NA \\
\hline Turquie et Levant & Nahr al Aouaj & Acanthobrama tricolor & Poissons & $\mathrm{CR}$ & NA & NA \\
\hline Turquie et Levant & Nahr al Aouaj & Oxynoemacheilus panthera & Poissons & EN & NA & NA \\
\hline Turquie et Levant & Nahr al Aouaj & Pseudophoxinus drusensis & Poissons & EN & NA & NA \\
\hline Turquie et Levant & Nahr al Kabir & Margaritifera homsensis & Mollusques & EN & oui & NA \\
\hline Turquie et Levant & Nahr al Kabir & Potomida littoralis & Mollusques & EN & NA & NA \\
\hline Turquie et Levant & Nahr al Kabir & Unio terminalis & Mollusques & VU & NA & NA \\
\hline Turquie et Levant & Nahr al Marqiya* & Pseudophoxinus hasani & Poissons & $\mathrm{CR}$ & oui & NA \\
\hline Turquie et Levant & Northern Coastal Streams of Syria* & Alburnus qalilus & Poissons & EN & oui & NA \\
\hline Turquie et Levant & Northern Coastal Streams of Syria* & Potomida littoralis & Mollusques & EN & NA & NA \\
\hline Turquie et Levant & Qweik* & Oxynoemacheilus tigris & Poissons & $\mathrm{CR}$ & oui & NA \\
\hline Turquie et Levant & Qweik* & Potomida littoralis & Mollusques & EN & NA & NA \\
\hline Turquie et Levant & Qweik* & Unio crassus & Mollusques & EN & NA & NA \\
\hline Turquie et Levant & Savrun catchment (Ceyhan drainage) & Capoeta erhani & Poissons & NA & oui & NA \\
\hline Turquie et Levant & Savrun catchment (Ceyhan drainage) & Cobitis evreni & Poissons & EN & oui & NA \\
\hline Turquie et Levant & Savrun catchment (Ceyhan drainage) & Hemigrammocapoeta culiciphaga & Poissons & NA & oui & NA \\
\hline Turquie et Levant & Savrun catchment (Ceyhan drainage) & Potomida littoralis & Mollusques & EN & NA & NA \\
\hline Turquie et Levant & Savrun catchment (Ceyhan drainage) & Unio crassus & Mollusques & EN & NA & NA \\
\hline Turquie et Levant & Savrun catchment (Ceyhan drainage) & Unio terminalis & Mollusques & VU & NA & NA \\
\hline Turquie et Levant & Spring of Barada (En Fidje) & Oxynoemacheilus panthera & Poissons & EN & oui & NA \\
\hline Turquie et Levant & Spring of Barada (En Fidje) & Pseudophoxinus syriacus & Poissons & $\mathrm{CR}$ & oui & NA \\
\hline Turquie et Levant & Sultan Sazlığı Marshes* & Aphanius danfordii & Poissons & $\mathrm{CR}$ & oui & NA \\
\hline Turquie et Levant & Sultan Sazlığı Marshes* & Pseudophoxinus elizavetae & Poissons & $\mathrm{CR}$ & oui & NA \\
\hline Turquie et Levant & Sultan Sazlığı Marshes* & Gyraulus argaeicus & Mollusques & VU & oui & NA \\
\hline Turquie et Levant & Sultan Sazlığı Marshes* & Pseudobithynia pentheri & Mollusques & NA & oui & NA \\
\hline Turquie et Levant & Sultan Sazlığı Marshes* & Unio crassus & Mollusques & EN & NA & NA \\
\hline Turquie et Levant & Upper Asi Lebanon* & Alburnus orontis & Poissons & VU & oui & NA \\
\hline Turquie et Levant & Upper Asi Lebanon* & Carasobarbus chantrei & Poissons & $\mathrm{NT}^{2}$ & NA & NA \\
\hline Turquie et Levant & Upper Asi Lebanon* & Pseudophoxinus kervillei & Poissons & EN & NA & NA \\
\hline Turquie et Levant & Upper Asi Lebanon* & Pseudophoxinus zeregi & Poissons & $\mathrm{LC}$ & NA & NA \\
\hline Turquie et Levant & Upper Asi Lebanon* & Pseudobithynia kathrinae & Mollusques & $\mathrm{CR}$ & oui & NA \\
\hline Turquie et Levant & Upper Asi Lebanon* & Pseudobithynia levantica & Mollusques & EN & oui & NA \\
\hline Turquie et Levant & Upper Dalaman & Alburnus demiri & Poissons & VU & oui & NA \\
\hline Turquie et Levant & Upper Dalaman & Cobitis phrygica & Poissons & EN & oui & NA \\
\hline Turquie et Levant & Upper Dalaman & Chondrostoma fahirae & Poissons & EN & oui & NA \\
\hline Turquie et Levant & Upper Dalaman & Torosia proschwitzi & Mollusques & NA & oui & NA \\
\hline
\end{tabular}


Tableau C : Espèces de déclenchement validées en Turquie et au Levant (suite).

\begin{tabular}{|c|c|c|c|c|c|c|}
\hline Sous-région & Nom de la ZCB ${ }^{1}$ & Espèces de déclenchement & Groupe & C1 & $\mathrm{C} 2$ & C3 \\
\hline Turquie et Levant & Upper Jordan Valley & Hemigrammocapoeta nana & Poissons & $\mathrm{NT}^{2}$ & NA & NA \\
\hline Turquie et Levant & Upper Jordan Valley & Luciobarbus longiceps & Poissons & EN & NA & NA \\
\hline Turquie et Levant & Upper Jordan Valley & Mirogrex terraesanctae & Poissons & NA & oui & NA \\
\hline Turquie et Levant & Upper Jordan Valley & Oxynoemacheilus leontinae & Poissons & NA & oui & NA \\
\hline Turquie et Levant & Upper Jordan Valley & Pseudophoxinus drusensis & Poissons & EN & oui & NA \\
\hline Turquie et Levant & Upper Jordan Valley & Pseudophoxinus kervillei & Poissons & EN & NA & NA \\
\hline Turquie et Levant & Upper Jordan Valley & Tristramella simonis & Poissons & NA & oui & NA \\
\hline Turquie et Levant & Upper Jordan Valley & Falsipyrgula barroisi & Mollusques & EN & oui & oui \\
\hline Turquie et Levant & Upper Jordan Valley & Potomida littoralis & Mollusques & EN & NA & NA \\
\hline Turquie et Levant & Upper Khabour & Barbus grypus & Poissons & VU & NA & NA \\
\hline Turquie et Levant & Upper Khabour & Carasobarbus kosswigi & Poissons & VU & NA & NA \\
\hline Turquie et Levant & Upper Khabour & Luciobarbus esocinus & Poissons & VU & NA & NA \\
\hline Turquie et Levant & Upper Khabour & Luciobarbus subquincunciatus & Poissons & $\mathrm{CR}$ & NA & NA \\
\hline Turquie et Levant & Upper Khabour & Melanopsis khabourensis & Mollusques & $\mathrm{CR}$ & oui & oui \\
\hline Turquie et Levant & Upper Khabour & Theodoxus cinctellus & Mollusques & NA & oui & NA \\
\hline Turquie et Levant & Upper Khabour & Unio crassus & Mollusques & EN & NA & NA \\
\hline Turquie et Levant & Upper Mujib* & Oxynoemacheilus insignis & Poissons & $\mathrm{NT}^{2}$ & NA & NA \\
\hline Turquie et Levant & Upper Mujib* & Pseudamnicola solitaria & Mollusques & EN & NA & NA \\
\hline Turquie et Levant & Wadi Karak Basin & Garra ghorensis & Poissons & EN & oui & NA \\
\hline Turquie et Levant & Wadi Karak Basin & Oxynoemacheilus insignis & Poissons & $\mathrm{NT}^{2}$ & NA & NA \\
\hline Turquie et Levant & Wadi Shuaib & Luciobarbus longiceps & Poissons & EN & NA & NA \\
\hline Turquie et Levant & Wadi Shuaib & Oxynoemacheilus insignis & Poissons & $\mathrm{NT}^{2}$ & NA & NA \\
\hline Turquie et Levant & Yarmuk basin* & Hemigrammocapoeta nana & Poissons & $\mathrm{NT}^{2}$ & NA & NA \\
\hline Turquie et Levant & Yarmuk basin* & Luciobarbus longiceps & Poissons & EN & NA & NA \\
\hline Turquie et Levant & Yarmuk basin* & Mirogrex terraesanctae & Poissons & NA & oui & NA \\
\hline Turquie et Levant & Yarmuk basin* & Oxynoemacheilus galilaeus & Poissons & $\mathrm{CR}$ & NA & NA \\
\hline Turquie et Levant & Yarmuk basin* & Oxynoemacheilus insignis & Poissons & $\mathrm{NT}^{2}$ & NA & NA \\
\hline Turquie et Levant & Yarmuk basin* & Pseudophoxinus kervillei & Poissons & EN & NA & NA \\
\hline Turquie et Levant & $\begin{array}{l}\text { Yarpuz and Hamus catchment (in } \\
\text { Ceyhan basin) }\end{array}$ & Capoeta erhani & Poissons & NA & oui & NA \\
\hline Turquie et Levant & $\begin{array}{l}\text { Yarpuz and Hamus catchment (in } \\
\text { Ceyhan basin) }\end{array}$ & Cobitis evreni & Poissons & EN & oui & NA \\
\hline Turquie et Levant & $\begin{array}{l}\text { Yarpuz and Hamus catchment (in } \\
\text { Ceyhan basin) }\end{array}$ & Hemigrammocapoeta culiciphaga & Poissons & NA & oui & NA \\
\hline Turquie et Levant & $\begin{array}{l}\text { Yarpuz and Hamus catchment (in } \\
\text { Ceyhan basin) }\end{array}$ & Pseudophoxinus zekayi & Poissons & VU & oui & NA \\
\hline Turquie et Levant & $\begin{array}{l}\text { Yarpuz and Hamus catchment (in } \\
\text { Ceyhan basin) }\end{array}$ & Potomida littoralis & Mollusques & EN & NA & NA \\
\hline Turquie et Levant & $\begin{array}{l}\text { Yarpuz and Hamus catchment (in } \\
\text { Ceyhan basin) }\end{array}$ & Unio crassus & Mollusques & EN & NA & NA \\
\hline Turquie et Levant & Zarqa River & Hemigrammocapoeta nana & Poissons & $\mathrm{NT}^{2}$ & NA & NA \\
\hline Turquie et Levant & Zarqa River & Oxynoemacheilus insignis & Poissons & $\mathrm{NT}^{2}$ & NA & NA \\
\hline Turquie et Levant & Zarqa River & Melanopsis ammonis & Mollusques & $\mathrm{CR}$ & NA & NA \\
\hline
\end{tabular}

1 N.D.T. : Les noms des sites ZCB figurant dans les tableaux sont en anglais. Toutefois, les noms des sites ZCB cités dans le corps de ce document ont été traduits en français en précisant leur nom anglais entre crochets (« [nom anglais : XXX] »), afin de permettre au lecteur de faire le lien avec les noms des sites en anglais figurant dans les tableauX correspondants et dans la base de données en ligne (WBDB, World Biodiversity DataBase).

2 Ces espèces sont incluses en tant qu'espèces de déclenchement de ZCB car, au moment de l'analyse, elles étaient répertoriées en tant qu'espèces menacées ou bien faisaient l'objet d'une proposition de reclassement en tant qu'espèces menacées. Le statut de ces espèces en tant qu'« espèces de déclenchement » de ZCB doit encore être clarifié 


\section{Annexe V. Ambassadeurs locaux}

Tableau D. Organisations ou personnes à envisager comme « ambassadeurs locaux » potentiels pour chaque ZCB (c.-à-d. pouvant mener des actions de conservation ou accroître la visibilité de la ZCB) ou ayant un impact ou des intérêts à l'égard de cette ZCB. * Site AZE.

\begin{tabular}{|c|c|c|c|}
\hline Sous-région & Pays & Nom de la ZCB ${ }^{1}$ & Ambassadeur local \\
\hline Balkans & Albanie & Butrint & $\begin{array}{l}\text { Ministere de l'environnement Albanais, Administration Forêts } \\
\text { et Eau, Parc national de Butrint }\end{array}$ \\
\hline Balkans & Bosnie-Herzégovine & Lake Busko & Ambassadeurs locaux à identifier \\
\hline Balkans & Bosnie-Herzégovine & Listica river and Mostarsko blato & $\begin{array}{l}\text { Gouvernement de Herzegovina-Neretva and West } \\
\text { Herzegovina county et Fédération de BiH, ONG internationaux } \\
\text { et locaux: EuroNatur, WWF, Nase Ptice, Lijepa Nasa, } \\
\text { EkoMost, Buna, etc. }\end{array}$ \\
\hline Balkans & Bosnie-Herzégovine & $\begin{array}{l}\text { Nevesinjsko polje, Gatacko polje, } \\
\text { Cernicko polje, Fatnicko polje and } \\
\text { Dabarsko polje }\end{array}$ & $\begin{array}{l}\text { Ministry of Spatial Planning Civil Engineering and Ecology, } \\
\text { Nature Protection Institute of RS, ONG: EuroNatur, WWF, } \\
\text { Nase Ptice, Centre for Environment from Banja Luka, Bastina } \\
\text { from Tomislavgrad, Lijepa Nasa from Capljina, Mocvara from } \\
\text { Capljina, EkoMost, etc. }\end{array}$ \\
\hline Balkans & Bosnie-Herzégovine & Part of the Neretva upper catchment & Ambassadeurs locaux à identifier \\
\hline Balkans & Bosnie-Herzégovine & $\begin{array}{l}\text { Part of the Neretva upper catchment } \\
\text { - eastern mid catchment }\end{array}$ & Ambassadeurs locaux à identifier \\
\hline Balkans & Bosnie-Herzégovine & Popovo polje and Trebisnjica & Ambassadeurs locaux à identifier \\
\hline Balkans & Bosnie-Herzégovine & $\begin{array}{l}\text { Tributaries of lower and middle } \\
\text { Neretva }\end{array}$ & $\begin{array}{l}\text { Gouvernement de Herzegovina-Neretva and West } \\
\text { Herzegovina county et Fédération de BiH, ONG internationaux } \\
\text { et locaux: EuroNatur, WWF, Nase Ptice, Lijepa Nasa, } \\
\text { EkoMost, Buna, etc. }\end{array}$ \\
\hline Balkans & Bosnie-Herzégovine & West Karst poljes & $\begin{array}{l}\text { Bosnia Ornithological Society, Energy Companies, EuroNatur, } \\
\text { Government Municipality, UNDP, WWF, Youth Centre }\end{array}$ \\
\hline Balkans & Croatie & Cetina river & $\begin{array}{l}\text { County public institution for nature protection of Split- } \\
\text { Dalmatian County, ONG locale Sunce }\end{array}$ \\
\hline Balkans & Croatie & Matica river and Bacina lakes & $\begin{array}{l}\text { County public institution for nature protection of Dubrovnik- } \\
\text { Neretva County, County public institution for nature protection } \\
\text { of Split-Dalmatian County, ONG locale Sunce }\end{array}$ \\
\hline Balkans & Croatie & Zrmanja river & $\begin{array}{l}\text { County public institution for nature protection of Zadar county, } \\
\text { Velebit Nature Park }\end{array}$ \\
\hline Balkans & Grèce & Acheron* & $\begin{array}{l}\text { Department of Aquaculture \& Fisheries, Technological } \\
\text { Educational Institute of Epirus, Institute of Inland Waters, } \\
\text { Hellenic Centre for Marine Research, Anavyssos, Grèce, } \\
\text { Managing Authority of Kalamas and Aherontas Rivers, Region } \\
\text { of Ipiros and management body }\end{array}$ \\
\hline Balkans & Grèce & Aggitis* $^{*}$ & $\begin{array}{l}\text { Region of East Macedonia Thrace (CIVIL ARCH), Water } \\
\text { Management Authority of Eastern Macedonia and Thrace }\end{array}$ \\
\hline Balkans & Grèce & Aliakmon Naoussa* & Water Management Authority of Central Macedonia \\
\hline Balkans & Grèce & Andros Tinos & $\begin{array}{l}\text { Region of Southern Aegean, Direction Water Resources } \\
\text { Management }\end{array}$ \\
\hline Balkans & Grèce & Arachthos & Institute of Marine Biological Resources and Inland Waters \\
\hline Balkans & Grèce & Arkadia Plateau & Préfecture d'Arcadia \\
\hline Balkans & Grèce & Chios & $\begin{array}{l}\text { Region of North Aegean Department of Environment and } \\
\text { Hydroeconomy RE of Chios }\end{array}$ \\
\hline Balkans & Grèce & Corfu Island (Kerkyra) & $\begin{array}{l}\text { Mr Stamatis Ginis, Fisheries Biologist, Region of Ionian - } \\
\text { Direction Water Resourses Management }\end{array}$ \\
\hline Balkans & Grèce & Crete Central South & Natural History Museum of Crète, WWF Crète \\
\hline Balkans & Grèce & Crete Eastern & $\begin{array}{l}\text { Natural History Museum of Crète, Region de Crète - Direction } \\
\text { Water Resourses Management, WWF Crète }\end{array}$ \\
\hline Balkans & Grèce & Crete North-west & Natural History Museum of Crète, WWF Crète \\
\hline Balkans & Grèce & Crete South-west* ${ }^{\star}$ & $\begin{array}{l}\text { Management Body of Samaria National Park, Natural History } \\
\text { Museum of Crète, Region of Crète Direction Water Resourses } \\
\text { Management, WWF Crète }\end{array}$ \\
\hline
\end{tabular}


Tableau D. Organisations ou personnes à envisager comme « ambassadeurs locaux » potentiels pour chaque ZCB (suite).

\begin{tabular}{|c|c|c|c|}
\hline Sous-région & Pays & Nom de la ZCB & Ambassadeur local \\
\hline Balkans & Grèce & Eastern Attica & Ambassadeurs locaux à identifier \\
\hline Balkans & Grèce & Euboea Manikiatis* & $\begin{array}{l}\text { Region of Sterea Ellada, Department of Environment and } \\
\text { Hydroeconomy RE of Euboea }\end{array}$ \\
\hline Balkans & Grèce & Evrotas & Ambassadeurs locaux à identifier \\
\hline Balkans & Grèce & Evrotas - Arniotikos & Ambassadeurs locaux à identifier \\
\hline Balkans & Grèce & Evrotas - Gytheio & Ambassadeurs locaux à identifier \\
\hline Balkans & Grèce & Ismaris-Vosvozis-Filiouris & $\begin{array}{l}\text { Management body for the National park (www. } \\
\text { fd.nestosvistonis.gr) }\end{array}$ \\
\hline Balkans & Grèce & Kalamas & Ambassadeurs locaux à identifier \\
\hline Balkans & Grèce & Karla* & $\begin{array}{l}\text { Management body of Karla -Mavrovounio - Velestino and } \\
\text { Kefalobryso }\end{array}$ \\
\hline Balkans & Grèce & Karpathos & Management Body of Karpathos-Saria \\
\hline Balkans & Grèce & Kastoria & Ambassadeurs locaux à identifier \\
\hline Balkans & Grèce & Kastraki & Ambassadeurs locaux à identifier \\
\hline Balkans & Grèce & Kephalonia and Ithaki & Region of Ionian Direction Water Resourses Management \\
\hline Balkans & Grèce & Kerkini & Management Authority of Kerkini Lake \\
\hline Balkans & Grèce & Krka drainage & $\begin{array}{l}\text { County public institution for nature protection of Sibenik - Knin } \\
\text { County, Public institution National park Krka }\end{array}$ \\
\hline Balkans & Grèce & Ladon & Région ouest de la Grèce \\
\hline Balkans & Grèce & Lake Kastrakiou & Ambassadeurs locaux à identifier \\
\hline Balkans & Grèce & $\begin{array}{l}\text { Lakes Limnothalassa Rodias, } \\
\text { Limnothalassa Tsoukaliou, } \\
\text { Limnothalassa Lagarou }\end{array}$ & Ambassadeurs locaux à identifier \\
\hline Balkans & Grèce & Lakes Trichonis and Lisimachia* & Ambassadeurs locaux à identifier \\
\hline Balkans & Grèce & Lesvos & $\begin{array}{l}\text { Basin Management Authority of Northern Aegean, ONG } \\
\text { Naftilus En Drasi, Region of North Aegean - Department of } \\
\text { Hydroeconomy RE of Lesvos }\end{array}$ \\
\hline Balkans & Grèce & Lower Acheloos & Ambassadeurs locaux à identifier \\
\hline Balkans & Grèce & Lower Alfeios & Ambassadeurs locaux à identifier \\
\hline Balkans & Grèce & Lower Axios & Axios-Loudias -Aliakmonas Management authority \\
\hline Balkans & Grèce & Magnisia* $^{*}$ & Ambassadeurs locaux à identifier \\
\hline Balkans & Grèce & Mornos & Ambassadeurs locaux à identifier \\
\hline Balkans & Grèce & Naxos & $\begin{array}{l}\text { Region of Southern Aegean, Direction Water Resources } \\
\text { Management }\end{array}$ \\
\hline Balkans & Grèce & Northern Korinthiakos & Ambassadeurs locaux à identifier \\
\hline Balkans & Grèce & Pamvotis Lake* & Management Body of Lake Pamvotis, Université de loannina \\
\hline Balkans & Grèce & Peloponnese Maleas* & Water Management Authority of Peloponnese \\
\hline Balkans & Grèce & Pineios Peloponnisou & Ambassadeurs locaux à identifier \\
\hline Balkans & Grèce & Pinios Thessalias & Ambassadeurs locaux à identifier \\
\hline Balkans & Grèce & Rhodes Island & $\begin{array}{l}\text { Forest department, ONG locale Aithria, Agro-Environmental } \\
\text { Research and Action Team, Moschous Stamatios, Region of } \\
\text { Southern Aegean, Direction Water Resources Management }\end{array}$ \\
\hline Balkans & Grèce & Spercheios* & $\begin{array}{l}\text { Managnement body of MT OITI National Park, Region of } \\
\text { Sterea Ellada Section of Environment and Hydroeconomy } \\
\text { of Pfthiotidi, Water Management Authority of the region of } \\
\text { Central Greece }\end{array}$ \\
\hline Balkans & Grèce & Tempi & Ambassadeurs locaux à identifier \\
\hline Balkans & Grèce & Thassos & $\begin{array}{l}\text { IBA care-taker, Water Management Authority of Eastern } \\
\text { Macedonia and Thrace }\end{array}$ \\
\hline Balkans & Grèce & Tragos & Ambassadeurs locaux à identifier \\
\hline Balkans & Grèce & Upper Alfeios & Ambassadeurs locaux à identifier \\
\hline Balkans & Grèce & Upper Aliakmon & Ambassadeurs locaux à identifier \\
\hline Balkans & Grèce & Upper Aoos & Ambassadeurs locaux à identifier \\
\hline Balkans & Grèce & Upper Kifissos* & Ambassadeurs locaux à identifier \\
\hline
\end{tabular}


Tableau D. Organisations ou personnes à envisager comme « ambassadeurs locaux » potentiels pour chaque ZCB (suite).

\begin{tabular}{|c|c|c|c|}
\hline Sous-région & Pays & Nom de la ZCB & Ambassadeur local \\
\hline Balkans & Grèce & Vistonis & $\begin{array}{l}\text { Management Body of National Park of Eastern Macedonia and } \\
\text { Thrace }\end{array}$ \\
\hline Balkans & Grèce & Volvi-Koronia & Ambassadeurs locaux à identifier \\
\hline Balkans & Grèce & Yliki-Paralimni-Kifissos* & Ambassadeurs locaux à identifier \\
\hline Balkans & Grèce & Zakynthos & Region of Ionian - Direction Water Resourses Management \\
\hline Balkans & Monténégro & Catchment surrounding Niksic ${ }^{*}$ & Ambassadeurs locaux à identifier \\
\hline Balkans & Monténégro, Albanie & Lake Skadar* & $\begin{array}{l}\text { Critical Ecosystem Partnership Fund (CEPF), Institute for } \\
\text { Nature Conservation in Albania (INCA), ONG Green Home } \\
\text { Montenegro }\end{array}$ \\
\hline Balkans & Albanie, ARY Macédoine & Lake Ohrid* & $\begin{array}{l}\text { Despina Kitanova. Society for Nature Conservation of } \\
\text { Macedonia, Université de Tirana, Albania }\end{array}$ \\
\hline Balkans & $\begin{array}{l}\text { Albanie, Grèce, ARY } \\
\text { Macédoine }\end{array}$ & Transboundary Prespa Park* & $\begin{array}{l}\text { PrespaNet representative organizations: Dr L. Melovski, } \\
\text { Macedonia Ecological Society; Dr S. Shumka, PPNEA and } \\
\text { Ms I. Koutseri, Society for the Protection of Prespa }\end{array}$ \\
\hline Balkans & Albanie, Monténégro & Lower Bojana river basin* & Ambassadeurs locaux à identifier \\
\hline Balkans & $\begin{array}{l}\text { Bosnie-Herzégovine, } \\
\text { Monténégro }\end{array}$ & Lake Bilecko & Ambassadeurs locaux à identifier \\
\hline Balkans & $\begin{array}{l}\text { Bosnie-Herzégovine, } \\
\text { Croatie }\end{array}$ & $\begin{array}{l}\text { Neretva delta and associated } \\
\text { springs/lakes }\end{array}$ & $\begin{array}{l}\text { County public institution for nature protection of Dubrovnik- } \\
\text { Neretva County, Croatian waters, Elektroprivreda HZHB RS } \\
\text { and HEP, Government of Herzegovina-Neretva and West } \\
\text { Herzegovina county and federation of BiH, Public enterprise } \\
\text { Nature park Hutovo blato }\end{array}$ \\
\hline Balkans & $\begin{array}{l}\text { Bosnie-Herzégovine, } \\
\text { Croatie }\end{array}$ & $\begin{array}{l}\text { Trebizat drainage including } \\
\text { Imotsko polje* }\end{array}$ & $\begin{array}{l}\text { County public institution for nature protection of Split- } \\
\text { Dalmatian County, Government of Herzegovina-Neretva } \\
\text { and West Herzegovina county and federation of } \mathrm{BiH} \text {, Local } \\
\text { engagement in endemic trout protection and local angling } \\
\text { society }\end{array}$ \\
\hline Balkans & Croatie, Slovénie, Italie & $\begin{array}{l}\text { Dragonija drainage in Slovénie } \\
\text { and Croatia, Reka river in } \\
\text { Slovénie and Timavo spring, } \\
\text { north of Trieste in Italie }\end{array}$ & $\begin{array}{l}\text { Community of Monfalcone/ltalie, Natura } 2000 \text { responsible } \\
\text { entities in Italy and Slovenia }\end{array}$ \\
\hline Balkans & Grèce, ARY Macédoine & Doirani* & Water directorate of Region of Central Macedonia \\
\hline Afrique du Nord & Algérie & Beni Belaid & $\begin{array}{l}\text { Laboratoire de Recherche et de Conservation des Zones } \\
\text { Humides, Université de Guelma, Ministère de l'Agriculture et } \\
\text { du Développement Rural, Ministère de l'environnement }\end{array}$ \\
\hline Afrique du Nord & Algérie & Hauts Plateaux & $\begin{array}{l}\text { Laboratoire de Recherche et de Conservation des Zones } \\
\text { Humides, Université de Guelma, Ministère de l'Agriculture et } \\
\text { du Développement Rural, Ministère de l'environnement }\end{array}$ \\
\hline Afrique du Nord & Algérie & Oued el Harrach & $\begin{array}{l}\text { Laboratoire de Recherche et de Conservation des Zones } \\
\text { Humides, Université de Guelma, Ministère de l'Agriculture et } \\
\text { du Développement Rural, Ministère de l'environnement }\end{array}$ \\
\hline Afrique du Nord & Algérie & Oued Zhour & Wilaya de Skikda \\
\hline Afrique du Nord & Algérie & Seybouse catchment & $\begin{array}{l}\text { Laboratoire de Recherche et de Conservation des Zones } \\
\text { Humides, Université de Guelma }\end{array}$ \\
\hline Afrique du Nord & Algérie & Tafna Catchment & $\begin{array}{l}\text { Laboratoire de Recherche et de Conservation des Zones } \\
\text { Humides, Université de Guelma }\end{array}$ \\
\hline Afrique du Nord & Algérie & Western Numidia & $\begin{array}{l}\text { Laboratoire de Recherche et de Conservation des Zones } \\
\text { Humides, Université de Guelma, Direction Générale des } \\
\text { Forêts (DGF) }\end{array}$ \\
\hline Afrique du Nord & Algérie & Western Numidia & Ministère de l'environnement \\
\hline Afrique du Nord & Maroc & Abid river downstream & $\begin{array}{l}\text { Agence du Bassin Hydraulique de l'Oum Er Rbia, Groupe } \\
\text { de Recherche pour la Protection des Oiseaux au Maroc } \\
\text { (GREPOM), Haut Commissariat aux Eaux et Forêts et à la } \\
\text { Lutte Contre la Désertification (HCEFLCD), Ministère de } \\
\text { l'intérieur / Direction Générale des Collectivités Locales } \\
\text { (DGCL), Ministère du tourisme, Muséum d'Histoire Naturelle } \\
\text { de Marrakech }\end{array}$ \\
\hline Afrique du Nord & Maroc & Arhreme river* & $\begin{array}{l}\text { Agence du Bassin Hydraulique du Souss Massa, Commune } \\
\text { d'Arhreme }\end{array}$ \\
\hline
\end{tabular}


Tableau D. Organisations ou personnes à envisager comme « ambassadeurs locaux » potentiels pour chaque ZCB (suite).

\begin{tabular}{|c|c|c|c|}
\hline Sous-région & Pays & Nom de la ZCB & Ambassadeur local \\
\hline Afrique du Nord & Maroc & Assif El Mal & $\begin{array}{l}\text { Haut Commissariat aux Eaux et Forêts et à la Lutte Contre la } \\
\text { Désertification (HCEFLCD) }\end{array}$ \\
\hline Afrique du Nord & Maroc & Assif El Mal east & $\begin{array}{l}\text { Agence du Bassin Hydraulique du Tensift (ABHT), Haut } \\
\text { Commissariat aux Eaux et Forêts et à la Lutte Contre la } \\
\text { Désertification (HCEFLCD), Ministère de l'intérieur / Direction } \\
\text { Générale des Collectivités Locales (DGCL) }\end{array}$ \\
\hline Afrique du Nord & Maroc & Assif Meloul river & $\begin{array}{l}\text { Commune of Imilchil, Groupe de Recherche pour la Protection } \\
\text { des Oiseaux au Maroc (GREPOM), Haut Commissariat } \\
\text { aux Eaux et Forêts et à la Lutte Contre la Désertification } \\
\text { (HCEFLCD), Université Moulay Ismail }\end{array}$ \\
\hline Afrique du Nord & Maroc & Lower Moulouya & $\begin{array}{l}\text { Agence du Bassin Hydraulique de la Moulouya, Amis de } \\
\text { Tafouralt, Communes locales, Haut Commissariat aux Eaux } \\
\text { et Forêts et à la Lutte Contre la Désertification (HCEFLCD), } \\
\text { Ministère de l'intérieur / Direction Générale des Collectivités } \\
\text { Locales (DGCL), Université Mohammed ler }\end{array}$ \\
\hline Afrique du Nord & Maroc & Lower Souss and tributaries & $\begin{array}{l}\text { Haut Commissariat aux Eaux et Forêts et à la Lutte Contre } \\
\text { la Désertification (HCEFLCD), ONG locales, Ministère de } \\
\text { l'intérieur / Direction Générale des Collectivités Locales } \\
\text { (DGCL), Agence du Bassin Hydraulique Sous Massa-Draa }\end{array}$ \\
\hline Afrique du Nord & Maroc & M'Goun river basin & $\begin{array}{l}\text { Haut Commissariat aux Eaux et Forêts et à la Lutte Contre } \\
\text { la Désertification (HCEFLCD), ONG locales, Ministère de } \\
\text { l'intérieur / Direction Générale des Collectivités Locales } \\
\text { (DGCL), Ministère du tourisme / Délégation regionale / Office } \\
\text { Régional de Mise en Valeur Agricole de Ouarzazate, Agence } \\
\text { du Bassin Hydraulique Sous Massa-Draa }\end{array}$ \\
\hline Afrique du Nord & Maroc & Middle N'Fiss river & $\begin{array}{l}\text { Haut Commissariat aux Eaux et Forêts et à la Lutte Contre la } \\
\text { Désertification (HCEFLCD) }\end{array}$ \\
\hline Afrique du Nord & Maroc & Middle Oum Er Rbia - Beni Mellal & $\begin{array}{l}\text { Agence du Bassin Hydraulique de l'Oum Er Rbia, Centre } \\
\text { de reproduction de carpes de Deroua (CNHP Azou), Haut } \\
\text { Commissariat aux Eaux et Forêts et à la Lutte Contre la } \\
\text { Désertification (HCEFLCD), ONG locales, Ministère de } \\
\text { l'intérieur / Direction Générale des Collectivités Locales } \\
\text { (DGCL) }\end{array}$ \\
\hline Afrique du Nord & Maroc & Middle Upper Moulouya & $\begin{array}{l}\text { Agence du Bassin Hydraulique de la Moulouya, Haut } \\
\text { Commissariat aux Eaux et Forêts et à la Lutte Contre la } \\
\text { Désertification (HCEFLCD), ONG locales, Ministère de } \\
\text { l'intérieur / Direction Générale des Collectivités Locales } \\
\text { (DGCL) }\end{array}$ \\
\hline Afrique du Nord & Maroc & Oued Amizmiz & $\begin{array}{l}\text { Haut Commissariat aux Eaux et Forêts et à la Lutte Contre la } \\
\text { Désertification (HCEFLCD), Agence du Bassin Hydraulique } \\
\text { Tensift , Parc national de Toubkal }\end{array}$ \\
\hline Afrique du Nord & Maroc & Oued Bouhlou & $\begin{array}{l}\text { Agence du Bassin Hydraulique de Sebou, Haut Commissariat } \\
\text { aux Eaux et Forêts et à la Lutte Contre la Désertification } \\
\text { (HCEFLCD), ONG locales à Taza, Ministère de l'intérieur / } \\
\text { Direction Générale des Collectivités Locales (DGCL) }\end{array}$ \\
\hline Afrique du Nord & Maroc & Oued Bouregreg & $\begin{array}{l}\text { Agence du Bassin Hydraulique du Bouregreg et de la Chaouia, } \\
\text { Bouregreg Management Agency, Haut Commissariat aux Eaux } \\
\text { et Forêts et à la Lutte Contre la Désertification (HCEFLCD), } \\
\text { ONG locales, Ministère de l'intérieur / Direction Générale des } \\
\text { Collectivités Locales (DGCL) }\end{array}$ \\
\hline Afrique du Nord & Maroc & Oued Imouzzer Kandar & $\begin{array}{l}\text { Agence du Bassin Hydraulique de Sebou, Groupe de } \\
\text { Recherche pour la Protection des Oiseaux au Maroc } \\
\text { (GREPOM), Haut Commissariat aux Eaux et Forêts et à la } \\
\text { Lutte Contre la Désertification (HCEFLCD), ONG locales } \\
\text { (e.g. SVT), Ministère de l'intérieur / Direction Générale des } \\
\text { Collectivités Locales (DGCL) }\end{array}$ \\
\hline Afrique du Nord & Maroc & Oued Ksob - Igrounzar & $\begin{array}{l}\text { Haut Commissariat aux Eaux et Forêts et à la Lutte Contre la } \\
\text { Désertification (HCEFLCD), Fondation Mohammed VI pour la } \\
\text { recherche et la protection de l'arganier }\end{array}$ \\
\hline Afrique du Nord & Maroc & Oued Lakhdar & $\begin{array}{l}\text { Agence du Bassin Hydraulique de l'Oum Er Rbia, Haut } \\
\text { Commissariat aux Eaux et Forêts et à la Lutte Contre la } \\
\text { Désertification (HCEFLCD), Agence du Bassin Hydraulique } \\
\text { Tensift }\end{array}$ \\
\hline
\end{tabular}


Tableau D. Organisations ou personnes à envisager comme « ambassadeurs locaux » potentiels pour chaque ZCB (suite).

\begin{tabular}{|c|c|c|c|}
\hline Sous-région & Pays & Nom de la ZCB & Ambassadeur local \\
\hline Afrique du Nord & Maroc & Oued Laou & $\begin{array}{l}\text { Haut Commissariat aux Eaux et Forêts et à la Lutte Contre la } \\
\text { Désertification (HCEFLCD), ONG locales (e.g. SVT), Ministère } \\
\text { de l'intérieur / Direction Générale des Collectivités Locales } \\
\text { (DGCL), Agence du Bassin Hydraulique Rhiss-Nekkor, } \\
\text { Université Abdelmalek Essaadi, Université Hassan II de } \\
\text { Casablanca }\end{array}$ \\
\hline Afrique du Nord & Maroc & Oued Massa & $\begin{array}{l}\text { Haut Commissariat aux Eaux et Forêts et à la Lutte Contre } \\
\text { la Désertification (HCEFLCD), ONG locales, cooperatives, } \\
\text { Ministère de la pêche, Ministère de l'Artisanat, Ministère du } \\
\text { tourisme, Agence du Bassin Hydraulique Sous Massa-Draa, } \\
\text { Université Ibnou Zohr }\end{array}$ \\
\hline Afrique du Nord & Maroc & Oued Tizguite and Oued Ouaslane & $\begin{array}{l}\text { Agence du Bassin Hydraulique de Sebou, Conseil municipal } \\
\text { d'Ifrane, Groupe de Recherche pour la Protection des Oiseaux } \\
\text { au Maroc (GREPOM), Haut Commissariat aux Eaux et Forêts } \\
\text { et à la Lutte Contre la Désertification (HCEFLCD), Ministère } \\
\text { de l'intérieur / Direction Générale des Collectivités Locales } \\
\text { (DGCL), Ministère de tourisme }\end{array}$ \\
\hline Afrique du Nord & Maroc & Oued Ziz Errachidia & $\begin{array}{l}\text { Agence du Bassin Hydraulique du Guir-Ziz-Rhéris- Errachidia, } \\
\text { Haut Commissariat aux Eaux et Forêts et à la Lutte Contre } \\
\text { la Désertification (HCEFLCD), ONG locales, Ministère de } \\
\text { l'intérieur / Direction Générale des Collectivités Locales } \\
\text { (DGCL) }\end{array}$ \\
\hline Afrique du Nord & Maroc & Saidia Coastal Plain & $\begin{array}{l}\text { Haut Commissariat aux Eaux et Forêts et à la Lutte Contre } \\
\text { la Désertification (HCEFLCD), Groupe de Recherche pour } \\
\text { la Protection des Oiseaux au Maroc (GREPOM), Agence du } \\
\text { Bassin Hydraulique Tensift }\end{array}$ \\
\hline Afrique du Nord & Maroc & Tifnout basin & $\begin{array}{l}\text { Agence du Bassin Hydraulique du Souss Massa, Collectivités } \\
\text { locales, Haut Commissariat aux Eaux et Forêts et à la Lutte } \\
\text { Contre la Désertification (HCEFLCD) }\end{array}$ \\
\hline Afrique du Nord & Maroc & Tigrigra stream & $\begin{array}{l}\text { Agence du Bassin Hydraulique de Sebou, Centre } \\
\text { d'Hydrobiologie et pisciculture d'Azrou, Groupe de Recherche } \\
\text { pour la Protection des Oiseaux au Maroc (GREPOM), Haut } \\
\text { Commissariat aux Eaux et Forêts et à la Lutte Contre la } \\
\text { Désertification (HCEFLCD), ONG locales, Ministère de } \\
\text { l'intérieur / Direction Générale des Collectivités Locales } \\
\text { (DGCL), Université Moulay Ismail }\end{array}$ \\
\hline Afrique du Nord & Maroc & Upper Dades & $\begin{array}{l}\text { Agence du Bassin Hydraulique du Draa, Agence du Bassin } \\
\text { Hydraulique Oum Er Rbia, Haut Commissariat aux Eaux et } \\
\text { Forêts et à la Lutte Contre la Désertification (HCEFLCD), } \\
\text { Ministère de l'intérieur / Direction Générale des Collectivités } \\
\text { Locales (DGCL) }\end{array}$ \\
\hline Afrique du Nord & Maroc & Upper Oued N'Fiss & $\begin{array}{l}\text { Haut Commissariat aux Eaux et Forêts et à la Lutte Contre la } \\
\text { Désertification (HCEFLCD), Ministère de l'intérieur / Direction } \\
\text { Générale des Collectivités Locales (DGCL), Agence du Bassin } \\
\text { Hydraulique Tensift }\end{array}$ \\
\hline Afrique du Nord & Maroc & Upper Oum Er Rbia & $\begin{array}{l}\text { Agence du Bassin Hydraulique de l'Oum Er Rbia, Associations } \\
\text { de pêcheurs, Groupe de Recherche pour la Protection des } \\
\text { Oiseaux au Maroc (GREPOM), Haut Commissariat aux Eaux } \\
\text { et Forêts et à la Lutte Contre la Désertification (HCEFLCD), } \\
\text { Ministère de l'intérieur / Direction Générale des Collectivités } \\
\text { Locales (DGCL) }\end{array}$ \\
\hline Afrique du Nord & Maroc & $\begin{array}{l}\text { Upper Oum Er Rbia above Kasba } \\
\text { Tadla }\end{array}$ & $\begin{array}{l}\text { Agence du Bassin Hydraulique de l'Oum Er Rbia, Le Haut } \\
\text { Commissariat aux Eaux et Forêts et à la Lutte Contre la } \\
\text { Désertification (HCEFLCD), Ministère de l'intérieur / Direction } \\
\text { Générale des Collectivités Locales (DGCL) }\end{array}$ \\
\hline Afrique du Nord & Tunisie & $\begin{array}{l}\text { Cap Serrat - Cap Blanc - Parc } \\
\text { national de l'Ichkeul }\end{array}$ & $\begin{array}{l}\text { Agence de Protection et d'Amenagement du Littoral (APAL), } \\
\text { Agence Nationale de Protection de l'Environnement (ANPE), } \\
\text { Direction Générale des Forêts (DGF), Association REACT }\end{array}$ \\
\hline Afrique du Nord & Tunisie & Maden River & $\begin{array}{l}\text { Direction Générale des Forêts (DGF), Association REACT, } \\
\text { Association des Amis des Oiseaux }\end{array}$ \\
\hline Afrique du Nord & Algérie, Maroc & Figuig oasis and Oued Saoura & $\begin{array}{l}\text { Laboratoire de Recherche et de Conservation des Zones } \\
\text { Humides, Université de Guelma, Ministère de l'Agriculture } \\
\text { et du Développement Rural,Ministère de l'environnement, } \\
\text { Patrimoine mondial - UNESCO }\end{array}$ \\
\hline
\end{tabular}


Tableau D. Organisations ou personnes à envisager comme « ambassadeurs locaux » potentiels pour chaque ZCB (suite).

\begin{tabular}{|c|c|c|c|}
\hline Sous-région & Pays & Nom de la ZCB & Ambassadeur local \\
\hline Afrique du Nord & Algérie, Tunisie & $\begin{array}{l}\text { El Kala - Les Tourbières de Dar } \\
\text { Fatma transboundary site }\end{array}$ & Ambassadeurs locaux à identifier \\
\hline Afrique du Nord & Maroc, Espagne (Melilla) & Le Grand Nador & $\begin{array}{l}\text { Agence du Bassin Hydraulique de la Moulouya, Haut } \\
\text { Commissariat aux Eaux et Forêts et à la Lutte Contre la } \\
\text { Désertification (HCEFLCD), ONG locales, Ministère de } \\
\text { la pêche, Ministère de l'intérieur / Direction Générale des } \\
\text { Collectivités Locales (DGCL), Agence du lac Nador, Université } \\
\text { Mohammed I }\end{array}$ \\
\hline Turquie et Levant & Jordanie & Amman & Ambassadeurs locaux à identifier \\
\hline Turquie et Levant & Jordanie & Upper Mujib* & $\begin{array}{l}\text { Development Zones Authority, Royal Society for Conservation } \\
\text { of Nature (RSCN) }\end{array}$ \\
\hline Turquie et Levant & Jordanie & Zarqa River & $\begin{array}{l}\text { Ministère de l'environnement, Royal Botanic Garden Jordan, } \\
\text { Royal Society for Conservation of Nature (RSCN) }\end{array}$ \\
\hline Turquie et Levant & Syrie & Middle Orontes & $\begin{array}{l}\text { Ministère de l'irrigation et Ministère de l'eau, SSCW Syrian } \\
\text { Society for the Conservation of Wildlife }\end{array}$ \\
\hline Turquie et Levant & Syrie & Nahr al Aouaj & $\begin{array}{l}\text { Ministère de l'irrigation et Ministère de l'eau, SSCW Syrian } \\
\text { Society for the Conservation of wildlife }\end{array}$ \\
\hline Turquie et Levant & Syrie & Nahr al Marqiya* & $\begin{array}{l}\text { Ministère de l'irrigation et Ministère de l'eau, SSCW Syrian } \\
\text { Society for the Conservation of wildlife }\end{array}$ \\
\hline Turquie et Levant & Syrie & Upper Khabour & Ambassadeurs locaux à identifier \\
\hline Turquie et Levant & Turquie & Aksu River & Ambassadeurs locaux à identifier \\
\hline Turquie et Levant & Turquie & Akyaka springs* & $\begin{array}{l}\text { Ministry of Forestry and Hydraulic affairs, UKAM (International } \\
\text { Karstic Water Resources Center - Hacettepe University) }\end{array}$ \\
\hline Turquie et Levant & Turquie & Bakirçay & Celal Bayar University \\
\hline Turquie et Levant & Turquie & Burdur lake and catchments (AZE) & $\begin{array}{l}\text { Burdur Municipality, Doga Dernegi, Ministry of Forestry and } \\
\text { Hydraulic Affairs }\end{array}$ \\
\hline Turquie et Levant & Turquie & Büyük Menderes river & $\begin{array}{l}\text { Adnan Menderes University, Akcay Sol Sahil Irrigation } \\
\text { Chamber, Aydin Memurlar Society, Buyuk Menderes Platform, } \\
\text { Coca Cola Life Plus Foundation, Ekosistemi Koruma ve Doga } \\
\text { Sevenler Dernegi, Pamukkale University, Soke Irrigation } \\
\text { Chamber, WWF Turkey }\end{array}$ \\
\hline Turquie et Levant & Turquie & Duden river* & $\begin{array}{l}\text { Antalya Municipality, DSI (State Hydraulic Works), UKAM } \\
\text { (International Karstic Water Resources Center - Hacettepe } \\
\text { University) }\end{array}$ \\
\hline Turquie et Levant & Turquie & Egirdir Lake catchment* & $\begin{array}{l}\text { Bizim Isparta Dernegi, Doga Dernegi, Egirdir Su Urunleri } \\
\text { Arastirma Enstitusu, WWF }\end{array}$ \\
\hline Turquie et Levant & Turquie & Gökdere (Yesildere) stream* & Ambassadeurs locaux à identifier \\
\hline Turquie et Levant & Turquie & Isikli/Civril lake and catchment* & Hacettepe University, Pamukkale University, WWF Turkey \\
\hline Turquie et Levant & Turquie & Karpuzcay stream* & Suleyman Demirel University - Fisheries Faculty \\
\hline Turquie et Levant & Turquie & Kopru Çay* & Directorate of National Parks \\
\hline Turquie et Levant & Turquie & Korkuteli and Elmali plains & Ambassadeurs locaux à identifier \\
\hline Turquie et Levant & Turquie & Küçük Menderes & $\begin{array}{l}\text { Aegean Birdwatching Society, Doga Dernegi, Ege University, } \\
\text { Sefrihisar municipality, WWF Turkey }\end{array}$ \\
\hline Turquie et Levant & Turquie & Lake Beysehir and catchments* & $\begin{array}{l}\text { Egirdir Su Urunleri Arastirma Enstitusu, Middle East Technical } \\
\text { University, Selcuk University, WWF }\end{array}$ \\
\hline Turquie et Levant & Turquie & Lake Iznik and catchment* & Istanbul University, Uludag University \\
\hline Turquie et Levant & Turquie & Lakes Acigöl and Salda & Hacettepe University, Municipality of Basmakci \\
\hline Turquie et Levant & Turquie & Lakes Aksehir - Eber system & Aksehir - Eber Golleri Koruma Birligi (protection chamber) \\
\hline Turquie et Levant & Turquie & Lower Gediz river & $\begin{array}{l}\text { Doga Dernegi, Dokuz Eylul University, Ege University, Izmir } \\
\text { Kus Cennetini Konima ve Gelistirme Birligi, Tour du Valat } \\
\text { Institut }\end{array}$ \\
\hline Turquie et Levant & Turquie & Manavgat River & $\begin{array}{l}\text { Antalya Orkidelerini ve Biyolojik Cesitliligini Koruma Dernegi, } \\
\text { Tema Antalya, UKAM (International Karstic Water Resources } \\
\text { Center - Hacettepe University) }\end{array}$ \\
\hline Turquie et Levant & Turquie & Middle and lower Seyhan river & $\begin{array}{l}\text { Cukurova University, Doga Arastimalari Dernegi, Ministry of } \\
\text { Forestry and Hydraulic affairs, WWF Turkey }\end{array}$ \\
\hline
\end{tabular}


Tableau D. Organisations ou personnes à envisager comme « ambassadeurs locaux » potentiels pour chaque ZCB (suite).

\begin{tabular}{|c|c|c|c|}
\hline Sous-région & Pays & Nom de la ZCB & Ambassadeur local \\
\hline Turquie et Levant & Turquie & $\begin{array}{l}\text { Savrun catchment (Ceyhan } \\
\text { drainage) }\end{array}$ & $\begin{array}{l}\text { Cukurova University, Ministry of Forestry and Hydraulic affairs, } \\
\text { Sutcu Imam University }\end{array}$ \\
\hline Turquie et Levant & Turquie & Sultan Sazligi Marshes* & $\begin{array}{l}\text { Doga Dernegi, Irrigation Unions, Ministry of Forestry and } \\
\text { Hydraulic affairs, State Hydraulic Works (DSI) }\end{array}$ \\
\hline Turquie et Levant & Turquie & Upper Dalman & Ambassadeurs locaux à identifier \\
\hline Turquie et Levant & Turquie & $\begin{array}{l}\text { Yarpuz and Hammus catchment (in } \\
\text { Ceyhan basin) }\end{array}$ & Sutcu Imam University \\
\hline Turquie et Levant & Jordanie, Israël & Wadi Karak Basin & $\begin{array}{l}\text { Ministère de l'environnement, Natural resources authority, } \\
\text { Royal Society for Conservation of Nature (RSCN), Water } \\
\text { Authority of Jordanie (WAJ) }\end{array}$ \\
\hline Turquie et Levant & Jordanie, Israël & Wadi Karak Basin & Ministère de l'Eau et de l'Irrigation \\
\hline Turquie et Levant & $\begin{array}{l}\text { Jordanie, Israël, } \\
\text { Territoires palestiniens } \\
\text { occupés }\end{array}$ & Central Jordanie River & $\begin{array}{l}\text { Bab Es-Salam Womens Cooperative, Friends of the Earth } \\
\text { Middle East (manage Sherahbeel lbn-Hasnah Park), Royal } \\
\text { Society for Conservation of Nature (RSCN) }\end{array}$ \\
\hline Turquie et Levant & Jordanie, Syrie & Yarmuk basin & $\begin{array}{l}\text { Ministère de l'environnement, SSCW Syrian Society for the } \\
\text { Conservation of Wildlife }\end{array}$ \\
\hline Turquie et Levant & Liban, Syrie & Litani River* & A Rocha, Al-Shouf Cedar Nature Reserve \\
\hline Turquie et Levant & $\begin{array}{l}\text { Territoires palestiniens } \\
\text { occupés }\end{array}$ & Jerico catchment & $\begin{array}{l}\text { Environment Quality Authority, IUCN ROWA, Ministère de } \\
\text { l'agriculture }\end{array}$ \\
\hline Turquie et Levant & Syrie, Jordanie, Israël & Lower Yarmouk & $\begin{array}{l}\text { Israel Nature and Parks Authority, Royal Society for } \\
\text { Conservation of Nature (RSCN), Waq'qas municipality }\end{array}$ \\
\hline Turquie et Levant & Syrie, Liban & Lake Homs (Qatinah) & Ambassadeurs locaux à identifier \\
\hline Turquie et Levant & Syrie, Liban & Nahr al Kabir & $\begin{array}{l}\text { Ministère de l'irrigation et Ministère de l'eau, SSCW Syrian } \\
\text { Society for the Conservation of wildlife }\end{array}$ \\
\hline Turquie et Levant & Syrie, Liban & Spring of Barada (En Fidje) & $\begin{array}{l}\text { Ministère de l'irrigation et Ministère de l'eau, SSCW Syrian } \\
\text { Society for the Conservation of wildlife }\end{array}$ \\
\hline Turquie et Levant & Syrie, Liban & Upper Asi Liban* & $\begin{array}{l}\text { Conventions internationales, ONG libanaises, Société pour la } \\
\text { Protection de la Nature - Liban }\end{array}$ \\
\hline Turquie et Levant & Syrie, Liban, Israël & Upper Jordanie Valley & SPNI Society for the protection of nature (Israel) \\
\hline Turquie et Levant & Syrie, Turquie & Northern Coastal Streams of Syrie & $\begin{array}{l}\text { Ministère de l'environnement, SSCW Syrian Society for the } \\
\text { Conservation of Wildlife }\end{array}$ \\
\hline Turquie et Levant & Turquie, Syrie & Lower Asi drainage & $\begin{array}{l}\text { Doga Dernegi, Subasi Bidwatching Society, TTKD Turkish } \\
\text { Society for the Protection of Nature }\end{array}$ \\
\hline Turquie et Levant & Turquie, Syrie & Qweik* $^{*}$ & $\begin{array}{l}\text { Doga Dernegi, TTKD Turkish Society for the Protection of } \\
\text { Nature }\end{array}$ \\
\hline Turquie et Levant & Turquie, Syrie, Iraq & Main stem of the Tigris River & Doga Dernegi, Nature Iraq \\
\hline
\end{tabular}

1 N.D.T. : Les noms des sites ZCB figurant dans les tableaux sont en anglais. Toutefois, les noms des sites ZCB cités dans le corps de ce document ont été traduits en français en précisant leur nom anglais entre crochets (« [nom anglais : XXX]»), afin de permettre au lecteur de faire le lien avec les noms des sites en anglais figurant dans les tableaux correspondants et dans la base de données en ligne (WBDB, World Biodiversity DataBase). 


\section{Annexe VI. ZCB proposées en raison de plantes et d'odonates de la sous-région de la Turquie et du Levant}

II existe relativement peu d'espèces de déclenchement de ZCB parmi les odonates (sept) et les plantes d'eau douce (cinq) dans la partie orientale du hotspot méditerranéen.

Les ZCB existantes et l'aire de répartition de l'ensemble des espèces de déclenchement composées d'odonates se chevauchent dans une certaine mesure. Leur répartition étant cartographiée pour situer les emplacements, la présence des espèces au sein d'une ZCB est considérée comme confirmée même si cela n'a pas été officiellement validé. Les ZCB supplémentaires proposées pour élargir la couverture de deux espèces d'odonates (Onychogomphus assimilis et Onychogomphus flexuosus, toutes les deux de catégorie Vulnérable et couvertes juste partiellement par le réseau des ZCB validées) comprennent les zones suivantes : i) le lac de Koycegiz et son bassin versant, et ; ii) le cours inférieur de l'Esen. Ces deux ZCB proposées sont à proximité de Dalaman dans la province de Mugla, sur la côte sud-ouest de la Turquie.

Les ZCB d'eau douce validées abritant des espèces de déclenchement composées d'odonates incluent les sites cidessous [noms anglais utilisés], énumérés par espèce :

- Brachythemis fuscopalliata (VU) - espèce actuellement présente dans les ZCB suivantes: Al Jabbul, Karpuzçay Stream, Köprü Çay, Lower Asi drainage, Middle and Lower Seyhan River, Middle Orontes, Northern Coastal Streams of Syria, et Upper Jordan Valley.

- Calopteryx hyalina (EN) - espèce actuellement présente dans les ZCB suivantes : Lake Homs (Qatinah), Litani River, Middle Orontes, Nahr al Kabir, Northern Coastal Streams of Syria, Spring of Barada (En Fidje), Upper Jordan Valley (même si la disparition de cette espèce est probable à cet endroit) et Nahr Al Aouaj.

- Calopteryx syriaca (EN) - espèce actuellement présente dans les ZCB suivantes: Amman, Central Jordan River, Jerico catchment, Lake Homs (Qatinah), Litani River, Lower Yarmouk, Middle Orontes, Nahr Al Aouaj, Nahr al Kabir, Spring of Barada (En Fidje), Upper Asi Lebanon, Upper Jordan Valley, Wadi Karak Basin, Wadi Shuaib et Zarqa River.

- Onychogomphus assimilis (VU) - espèce actuellement présente dans les ZCB suivantes : Düden River, Köprü Stream, et Middle and lower Seyhan River.

- Onychogomphus flexuosus (VU) - espèce actuellement présente dans les ZCB suivantes : Büyük Menderes River, Jerico catchment et Lower Yarmouk.

- Onychogomphus macrodon (VU) - espèce actuellement présente dans les ZCB suivantes : Central Jordan River, Lake Homs (Qatinah), Lower Asi drainage, Middle Orontes, Upper
Jordan Valley et Upper Khabour (même si la disparition de cette espèce est probable à cet endroit).

Concernant les plantes de milieux humides et les plantes aquatiques, trois nouvelles ZCB d'eau douce (l'une étant un site AZE) doivent être délimitées et validées pour garantir que toutes les espèces de déclenchement sont intégrées au réseau des ZCB. Deux espèces sont déjà couvertes par des ZCB validées : i) l'espèce Rumex bithynicus (VU) qui est partiellement couverte par la ZCB du lac d'Iznik et son bassin versant [nom anglais : Lake Iznik and Catchment], et ii) l'espèce Thermopsis turcica (CR) qui est intégralement couverte par la ZCB du Système des lacs Akşehir - Eber [nom anglais : Lakes Akşehir - Eber System] (qui répondrait aux critères d'admission en tant que site $A Z E$ en raison de cette espèce). Deux (voire trois) de ces ZCB d'eau douce proposées sont déjà couvertes par des ZCB « terrestres » existantes (voir précisions ci-dessous).

Les ZCB supplémentaires proposées en raison de plantes de milieux humides et de plantes aquatiques sont les suivantes :

Le bassin d'Ömerli (41.036 N, 29.374 E) - les parties supérieures de ce bassin versant se situent juste à l'intérieur du hotspot du bassin méditerranéen. Ce site répondrait aux critères d'admission en tant que site AZE en raison de l'amsonie Amsonia orientalis (CR), une espèce dont la présence n'a été enregistrée qu'en Grèce (catégorie "Peut-être éteint ») et dans le nord-ouest de la Turquie où elle n'est observée que dans le bassin d'Ömerli. Les autres emplacements connus dans le nord-ouest de la Turquie ont été perdus. Cette ZCB d'eau douce proposée est couverte par une Zone importante pour les plantes (ZIP) existante, nommée « Ömerli Havzasi ».

Le Djebel Druze (32.697 N, 36.729 E) — un massif dans le sud de la Syrie. Ce site répondrait aux critères d'admission en tant que site AZE en raison de l'isoète de l'Olympe (Isoetes olympica) (CR), cette espèce ayant peut-être disparu de son unique autre emplacement connu, en Bithynie à Bursa (mont Olympe) en Turquie. Ce site est également couvert par une ZIP existante nommée « Jabal al Arab/Jabal Druze ».

Une ou plusieurs parties du versant occidental de la chaîne montagneuse libanaise $(33.785$ N, 35.656 E). Ce site répondrait aux critères d'admission en tant que $\mathrm{ZCB}$ en raison de l'espèce Ranunculus schweinfurthii (VU) qui est endémique à cette région. II existe un certain nombre de ZCB existantes dans cette région (par exemple, la réserve naturelle des cèdres du Chouf et la vallée du fleuve de Beyrouth) donc cette espèce est peut-être déjà incluse dans l'une ou plusieurs d'entre elles. 



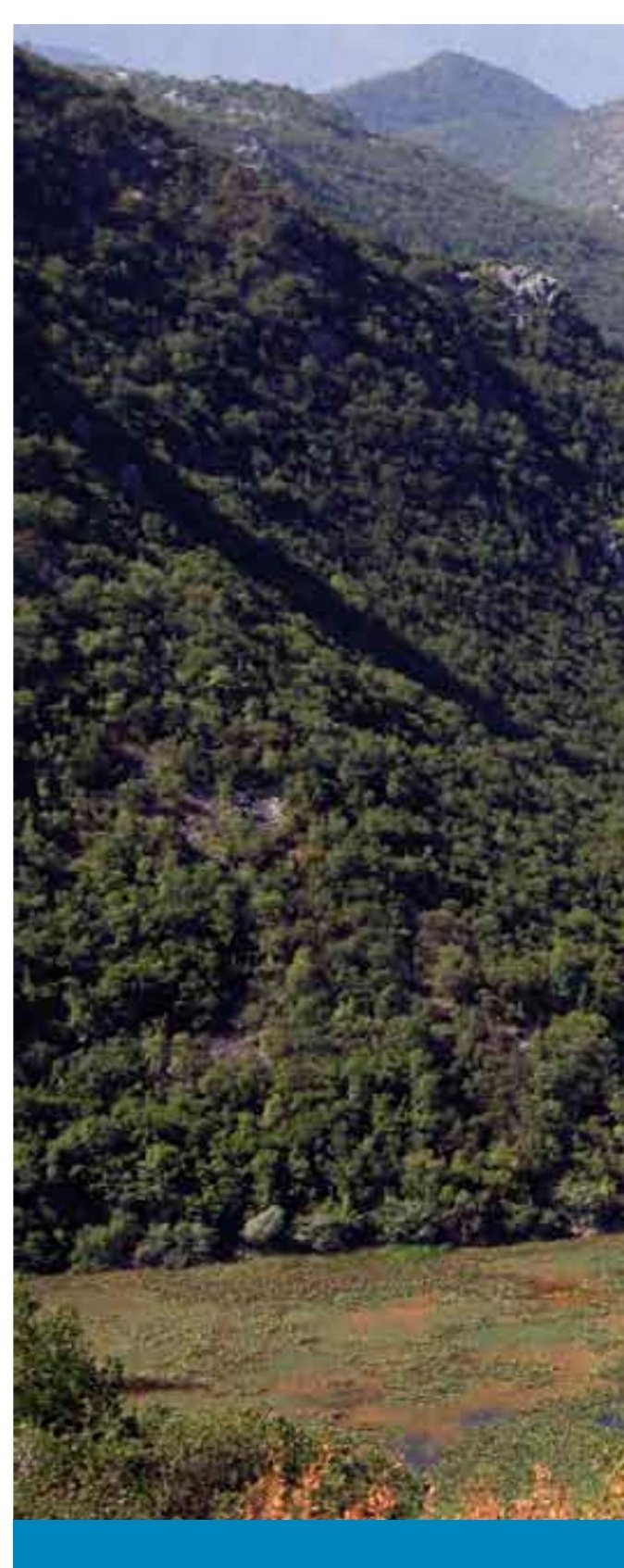

UNION INTERNATIONALE POUR LA

CONSERVATION DE LA NATURE

SIĖGE MONDIAL

Rue Mauverney 28

1196 Gland, Suisse

Tel +41 229990000

Fax +41 229990002

www.iucn.org 